\title{
WestVirginiaUniversity
}

THE RESEARCH REPOSITORY @ WVU

Graduate Theses, Dissertations, and Problem Reports

2013

\section{Composite Multifunctional Lithium Ion Batteries}

Joshua Mullenax

West Virginia University

Follow this and additional works at: https://researchrepository.wvu.edu/etd

\section{Recommended Citation}

Mullenax, Joshua, "Composite Multifunctional Lithium Ion Batteries" (2013). Graduate Theses,

Dissertations, and Problem Reports. 660.

https://researchrepository.wvu.edu/etd/660

This Thesis is protected by copyright and/or related rights. It has been brought to you by the The Research Repository @ WVU with permission from the rights-holder(s). You are free to use this Thesis in any way that is permitted by the copyright and related rights legislation that applies to your use. For other uses you must obtain permission from the rights-holder(s) directly, unless additional rights are indicated by a Creative Commons license in the record and/ or on the work itself. This Thesis has been accepted for inclusion in WVU Graduate Theses, Dissertations, and Problem Reports collection by an authorized administrator of The Research Repository @ WVU. For more information, please contact researchrepository@mail.wvu.edu. 


\title{
Composite Multifunctional Lithium Ion Batteries
}

\author{
Joshua Mullenax
}
Thesis submitted to the College of Engineering and Mineral Resources at West Virginia University
in partial fulfillment of the requirements
for the degree of
Master of Science
in
Mechanical Engineering

\author{
Edward M. Sabolsky, Ph.D. Chair \\ Patrick Browning, PhD. \\ Wade W. Huebsch, PhD.
}

Department of Mechanical and Aerospace Engineering

Morgantown, West Virginia

2013

Keywords: Multifunctional Lithium Ion Battery, Carbon Fiber Electrochemical Performance, Structural Battery Architecture

Copyright 2013 Joshua Mullenax 


\section{ABSTRACT \\ Composite Multifunctional Lithium Ion Batteries}

\section{Joshua Mullenax}

The goal of this work was to design a battery that serves as the structural material as well as the power source for a transportation vehicle. The combination of both mechanical and electrochemical aspects within one material defines the component as a multifunctional material, or in this case, a multifunctional battery. The design of the composite multifunctional batteries for optimal performance involves the proper selection of the materials, architecture, and electrical interconnection. The ultimate goal is to incorporate a battery with a continuous composite fibrous fabric within the structured composite skin of a vehicle (such as an automobile or aircraft).

This work included a survey of the electrochemical potential of multiple composite fabrics, such as fiberglass and modified carbon fiber, as substitutions for the electrode and separator materials of the battery. Each modified material was examined by a typical cyclic voltage-capacity testing in a traditional button cell platform. The performance for the use of the modified carbon fibers as the anode was then compared to the performance of conventional lithium ion materials to see which of the pretreatments improved the carbon fiber's performance. In addition to this electrochemical testing, flexure and tensile mechanical data of various geometries of perforated pouch cell architectures were examined under varying structural loads while the battery was electrochemically tested. In-situ testing of structural cells was conducted to determine the best configuration for object specific structural batteries. 


\section{Table of Contents}

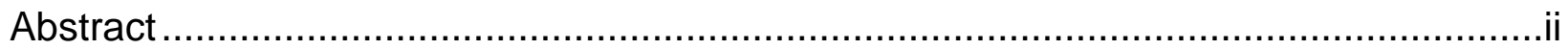

Table of Contents ............................................................................................

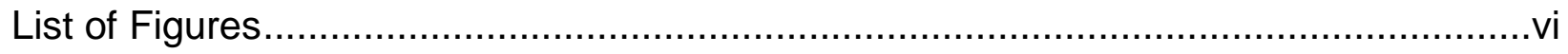

List of Tables

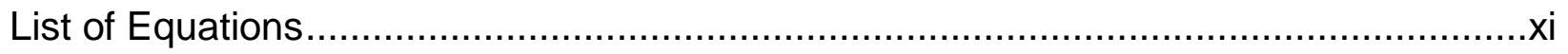

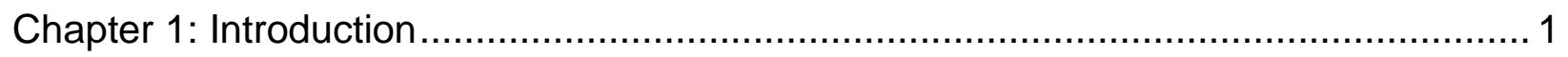

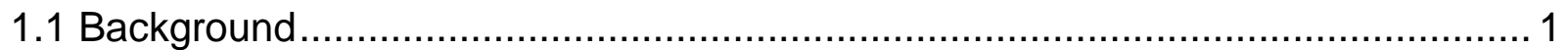

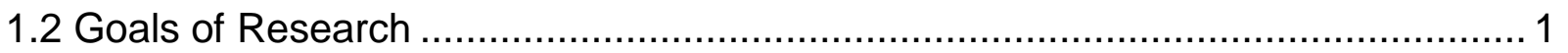

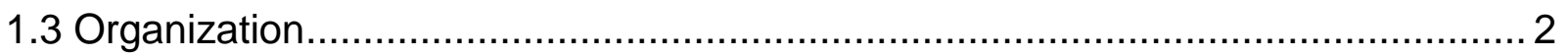

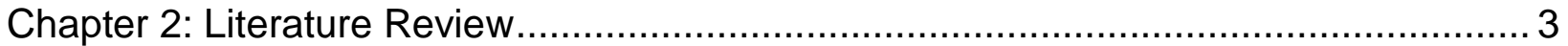

2.1 General Description of a Battery ..............................................................

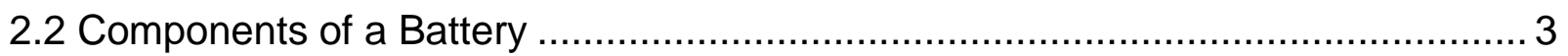

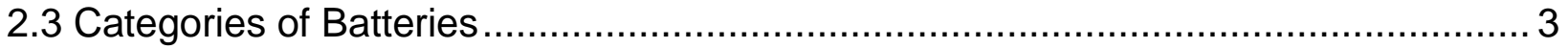

2.3.1 Primary Battery ……………………………………………………

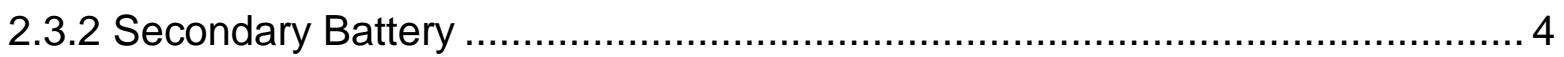

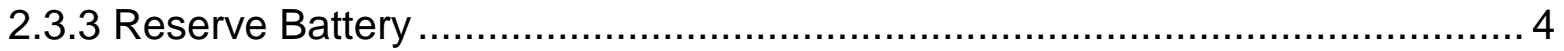

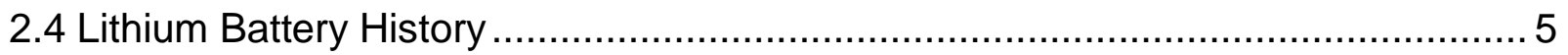

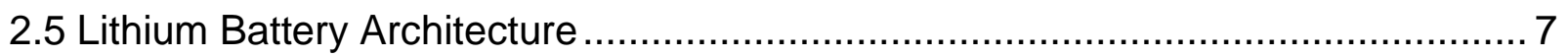

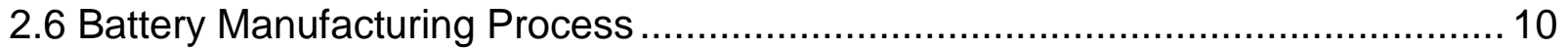

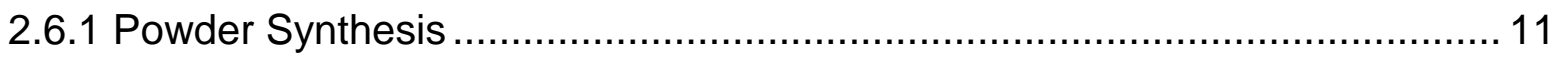

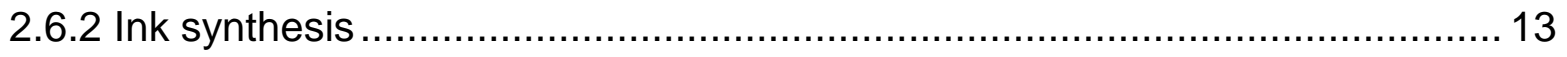

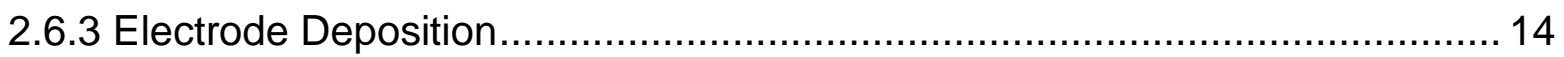

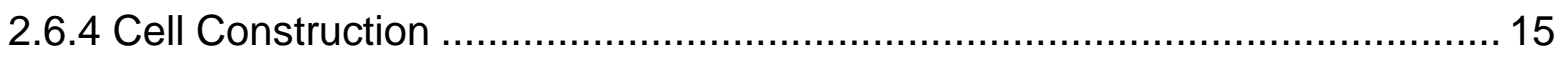

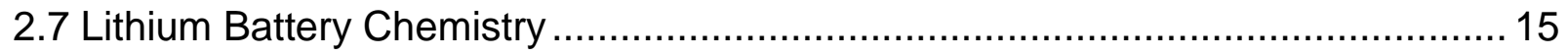

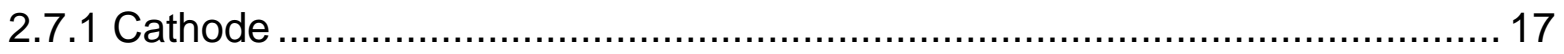

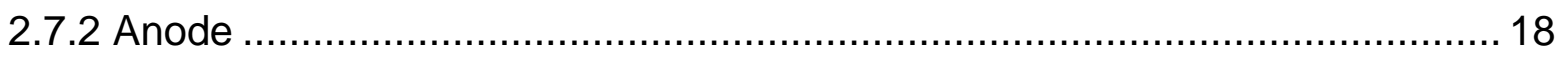

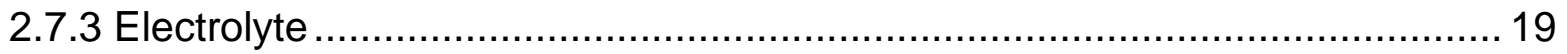

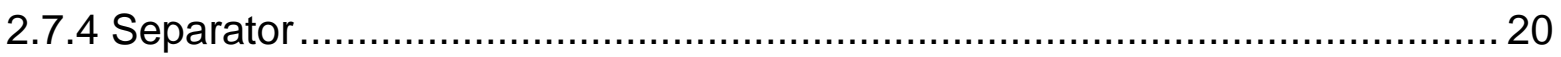

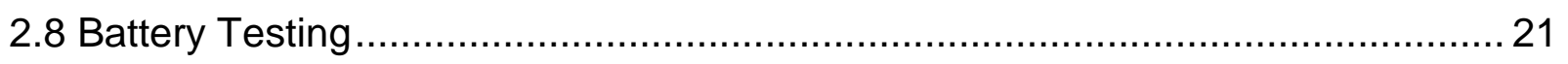




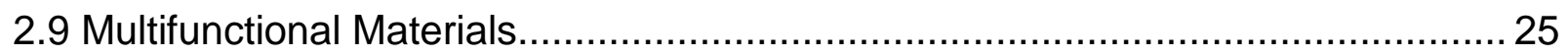

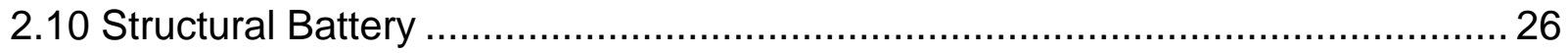

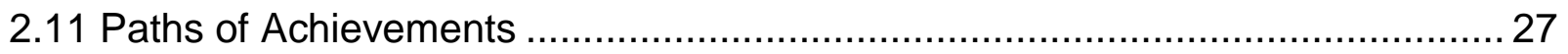

Chapter 3: Fabrication of the Novel Lithium Ion Cells ................................................ 29

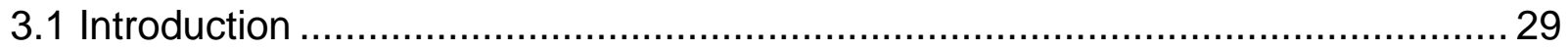

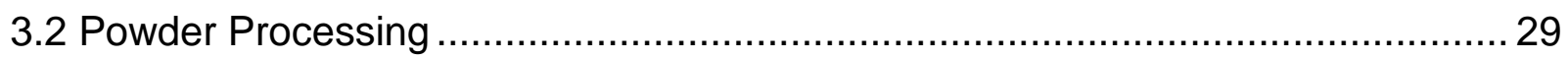

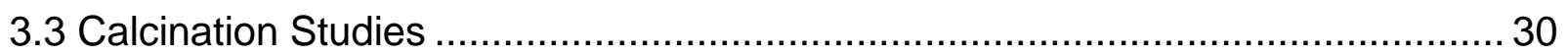

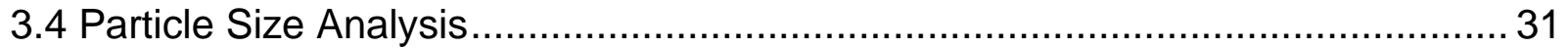

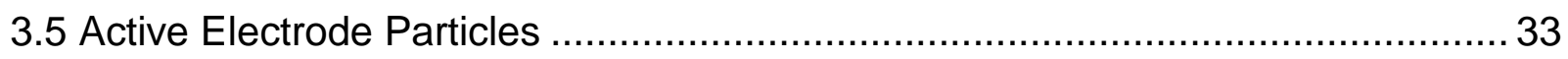

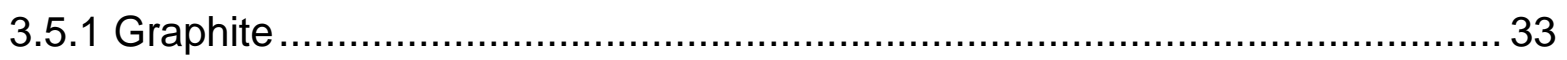

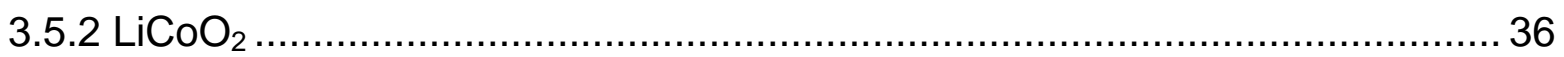

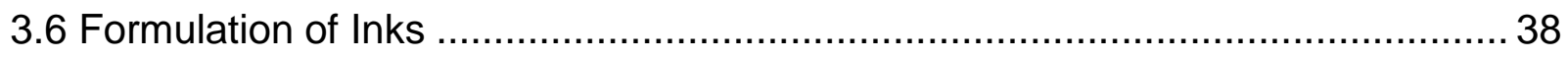

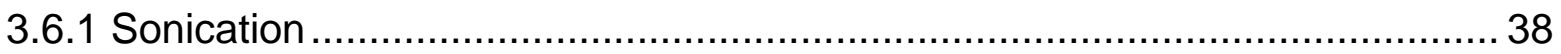

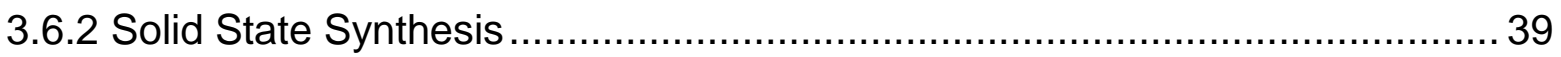

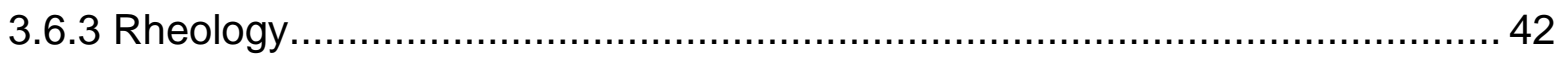

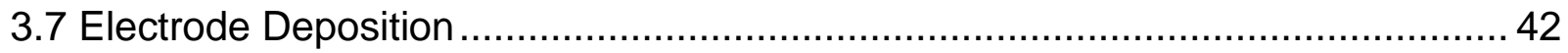

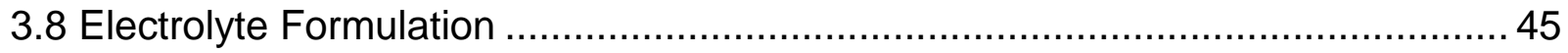

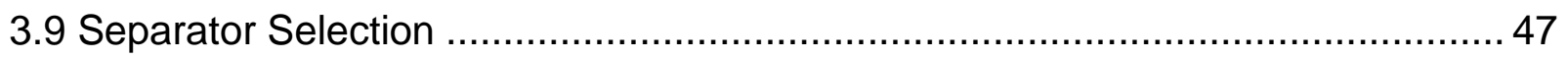

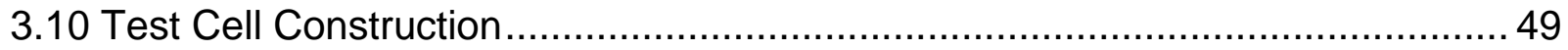

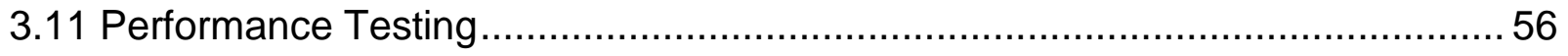

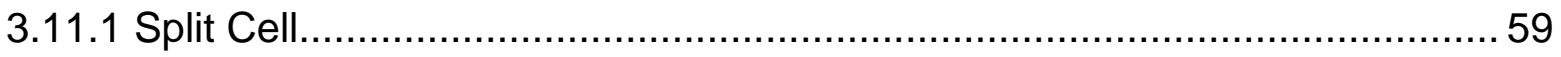

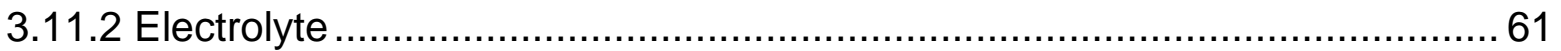

3.11.3 Preliminary Composite Performance Data................................................ 63

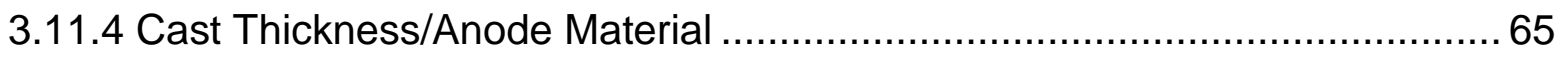

3.11.5 Solid Electrolyte Interface Formation ..................................................... 71

3.11.6 Off-the-Shelf Powder vs. In-House Synthesized Powder............................ 73

Chapter 4: Electrochemical Evaluation of Multifunctional Materials ............................. 76

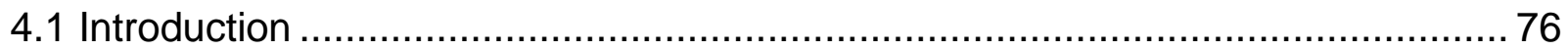

4.2 Carbon Fiber Experimental Treatments ……................................................ 77

4.3 Characterization of Carbon Fiber Treatments ................................................ 79

4.4 Carbon Fiber Electrochemical Performance Testing......................................... 85 


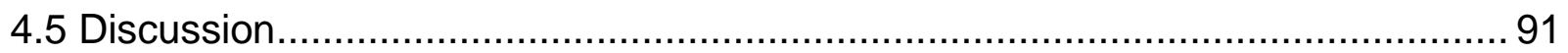

Chapter 5: Mechanical Evaluation of Structural Cells ............................................... 93

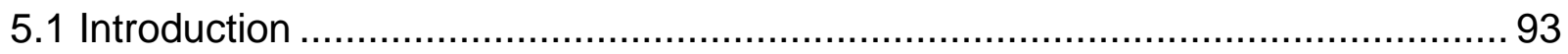

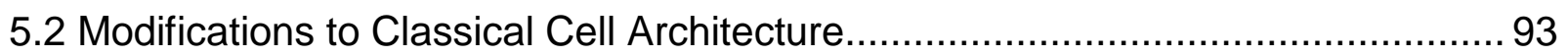

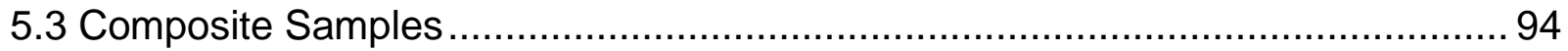

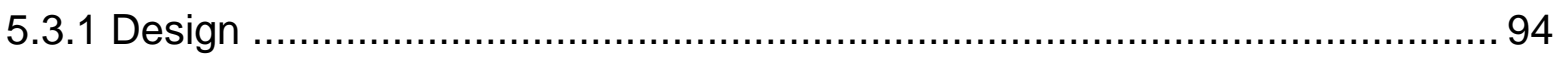

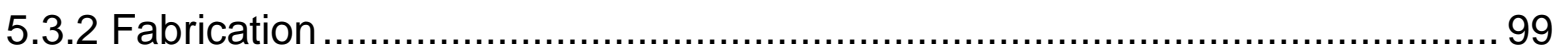

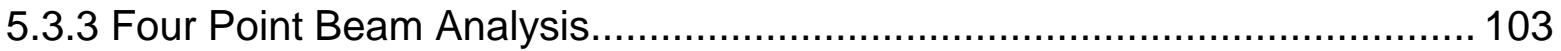

5.3.4 In-Situ Multifunctional Battery Four Point Beam Analysis ......................... 107

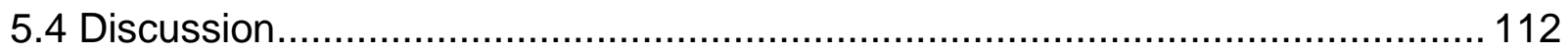

Chapter 6: Conclusions and Future Work ……................................................. 114

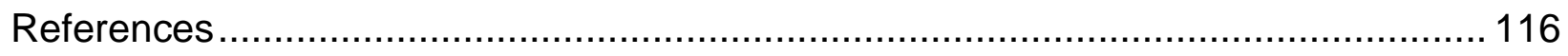

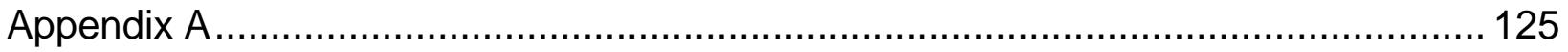




\section{List of Figures}

Figure 1 - Comparison of the volumetric and gravimetric energy density with different

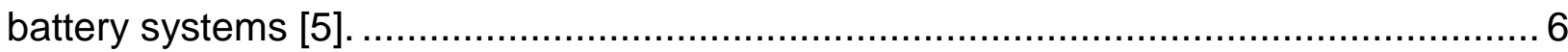

Figure 2 - Diagram of a classical spun cylindrical cell type battery construction [8] ....... 7

Figure 3 - Laminated lithium prismatic cell layers [2]................................................ 8

Figure 4 - Illustration of polymer enhanced electrodes in a pouch battery [11] ............. 9

Figure 5 - Demonstration of the swelling effect in pouch batteries............................. 9

Figure 6 - Cross sectional view of common layers in a coin cell [4] .......................... 10

Figure 7 - General overview of the several processes involved in manufacturing lithium

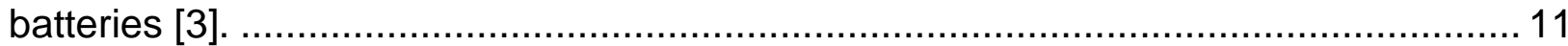

Figure 8 - SEM micrograph of large grain powders made from a classical solid state

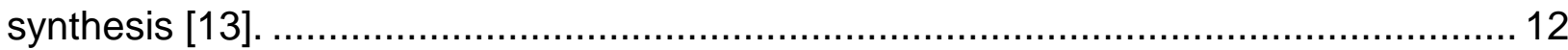

Figure 9 - SEM micrograph of $\mathrm{LiCoO}_{2}$ powders obtained from a hydrothermal synthesis

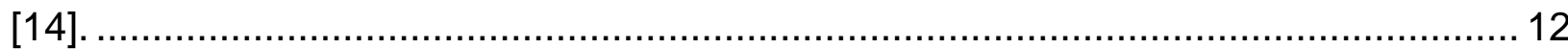

Figure 10 - SEM micrograph of $\mathrm{LiCoO}_{2}$ powders derived from a sol gel method [12] ... 13 Figure 11 - Variety of secondary type lithium ion battery components [2] .................. 16

Figure 12 - Charge and discharge chemistry of a typical lithium ion battery [24]. ........ 17 Figure 13 - Layered structure of $\mathrm{LiCoO}_{2}$ showing the lithium ions between the

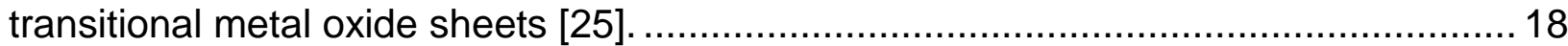

Figure 14 - Microstructure of a micro porous polymer film manufactured by (A) dry

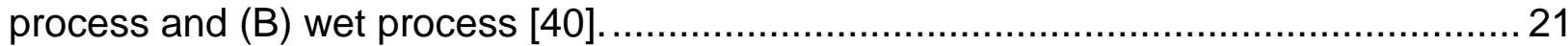
Figure 15 - Schematic of the formation of the SEI layer by electrolyte decomposition of

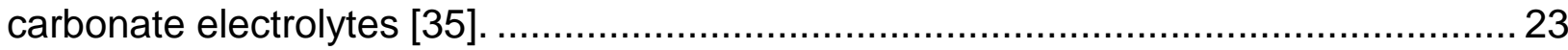

Figure 16 - Specific capacity and coulombic efficiency trend vs. number of cycles of a

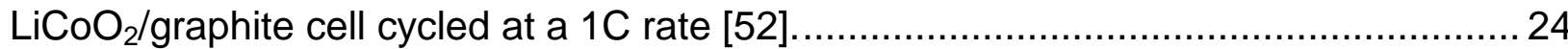

Figure 17 - Conventional lithium ion cathode discharge performance compared to a structural electrode modified with carbon nanofiber [11] ..........................................2. 25

Figure 18 - DARPA Wasp micro-air vehicle with lithium ion pouch cells integrated into

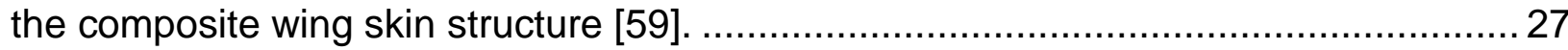
Figure 19 - A schematic of the first battery architecture showing a cell embedded within a laminate composite of continuous fiberglass and carbon fiber weaves running through

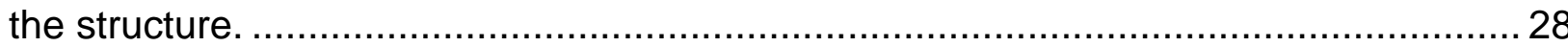

Figure 20 - A schematic cross section of the second battery architecture, which consists of reinforcement vias passing through the active components within a pouch battery; therefore, allowing for further shear reinforcement through the interior of the battery

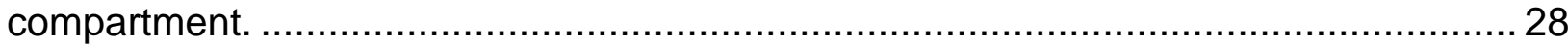

Figure 21 - Overlay of X-Ray diffraction scans of $\mathrm{LiCoO}_{2}$ cathode powder calcinated at

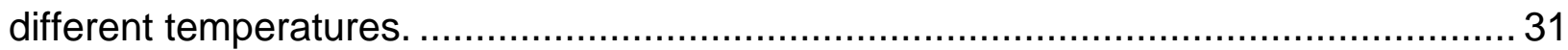

Figure 22 - Average particle size as a function of milling time from $0.5-9$ hours......... 32 
Figure 23 - Scanning electron micrographs of $(A)$ natural graphite powder and (B) synthetic graphite powder.

Figure 24 - X-Ray diffraction pattern overlay of natural graphite and synthetic graphite powders.

Figure 25 - Scanning electron micrographs of $(A)$ in-house synthesized $\mathrm{LiCoO}_{2}$ and (B) off the shelf $\mathrm{LiCoO}_{2}$ powder. 36

Figure 26 - X-Ray diffraction pattern overlay of the in-house synthesized powder compared to an off the shelf powder. 37

Figure 27 - Scanning electron micrographs of conductive additives (A) carbon black (Timcal, Super C45) and (B) carbon nanofiber (Pyrograf Products, PR-19-XT-LHT) .... 40 Figure 28 - Tape casted cathode electrodes for lithium ion batteries. (A) Unsuccessfully fabricated electrode and (B) successfully manufactured electrode. 44 Figure 29 - Micrographs of unused tri-layer PP/PE/PP separators (A) MTI Corporation battery film (B) Celgard 2325 film. 48

Figure 30 - Micrographs of cycled tri-layer PP/PE/PP separators (A) MTI Corporation

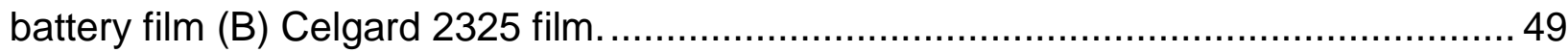

Figure 31 - Three electrode split cell testing apparatus......................................... 50

Figure 32 - C-200 carbon dioxide programmable laser cutter system......................... 51

Figure 33 - Picture of coin cell fabrication pieces in glovebox................................. 52

Figure 34 - Electrolyte mixing process conducted in inert atmosphere of glovebox. .....53 Figure 35 - Schematic of internal layered components comprised in performance test

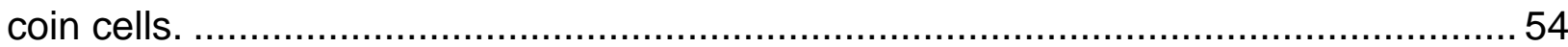

Figure 36 - Battery arbor press with CR2032 coin cell dies, ready to crimp coin cell. ... 55 Figure 37 - Successfully epoxy sealed coin cell followed by a failed epoxy sealed coin cell with electrolyte leakage from gaseous buildup. 56 Figure 38 - Initial battery test stand consisting of BK Precision's 9121A programmable DC power supply and 8500 programmable DC electronic load cycling a three electrode split cell in a battery testing box. 58

Figure 39 - MTI Corporation 8 channel battery analyzer testing stand setup............... 59 Figure 40 - Baseline performance cycle of classical lithium ion architecture in a three electrode split cell.

Figure 41 - Electrolyte performance data consisting of the coulombic efficiency overlaid with the normalized discharge capacitance of each electrolyte. 62 Figure 42 - Electrochemical testing data with different architectures including a novel lithium ion cell, a substituted carbon fiber anode cell, and a substituted fiberglass separator with carbon fiber cell. 64 Figure 43 - Normalized initial coin cell capacitance comparing different anode formulations over wet tape casting thicknesses.

Figure 44 - SEM micrographs of the composite structural electrode, including conductive additives of carbon black and carbon nanofibers as a structural 
reinforcement $(A)$ cathode electrode and $(B)$ structural composite cathode manually fractured to illustrate carbon nanofiber's structural reinforcement.

Figure 45 - SEM micrographs of different anode formulations (A) natural graphite (B) synthetic graphite (C) natural graphite with carbon additives (D) synthetic graphite with

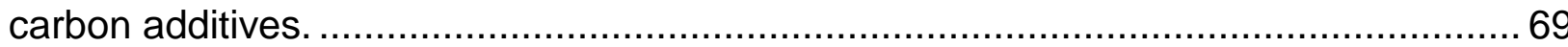

Figure 46 - Cycling data overlaying the capacity fade of different anode formulations.. 70 Figure 47 - Solid electrolyte interface cycling formation tests................................... 72 Figure 48 - Rate testing comparing in-house synthesized LiCoO2 powder to an off the shelf (Sigma Aldrich) $\mathrm{LiCoO}_{2}$ powder at different charge and discharge rates. The key represents the charge (C) and discharge (D) rate related to the capacitance of the cell.

Figure 49 - SEM micrograph and corresponding XPS spectra deconvolution analysis of unmodified T-300 commercial grade fibers. 79 Figure 50 - SEM micrograph and corresponding XPS spectra deconvolution analysis of desized T-300 commercial grade fibers by a heat treatment of $200^{\circ} \mathrm{C}$ 80 Figure 51 - SEM micrograph and corresponding XPS spectra deconvolution analysis of desized T-300 commercial grade fibers by a heat treatment of $400^{\circ} \mathrm{C}$ 80 Figure 52 - SEM micrograph and corresponding XPS spectra deconvolution analysis of desized T-300 commercial grade fibers by an inert atmospheric heat treatment at $200^{\circ} \mathrm{C}$.

Figure 53 - SEM micrograph and corresponding XPS spectra deconvolution analysis of desized T-300 commercial grade fibers by an inert atmospheric heat treatment at $400^{\circ} \mathrm{C}$

Figure 54 - SEM micrograph and corresponding XPS spectra deconvolution analysis of desized T-300 commercial grade fibers by a reducing atmospheric heat treatment at $200^{\circ} \mathrm{C}$.

Figure 55 - SEM micrograph and corresponding XPS spectra deconvolution analysis of desized T-300 commercial grade fibers by a reducing atmospheric heat treatment at $400^{\circ} \mathrm{C}$.

Figure 56 - SEM micrograph and corresponding XPS spectra deconvolution analysis of desized T-300 commercial grade fibers by a $1 \mathrm{M}$ hydrochloric acid treatment. Figure 57 - SEM micrograph and corresponding XPS spectra deconvolution analysis of desized T-300 commercial grade fibers by a $1 \mathrm{M}$ nitric acid treatment. Figure 58 - SEM micrograph and corresponding XPS spectra deconvolution analysis of desized T-300 commercial grade fibers by a $1 \mathrm{M}$ sulfuric acid treatment. 84 Figure 59 - Cathode based specific capacity electrochemical performance data of the different pretreatments conducted to the carbon fiber anode.

Figure 60 - Anode based specific capacity electrochemical performance data of the different pretreatments conducted to the carbon fiber anode. 
Figure 61 - Anode based specific capacity electrochemical performance data of PAN and Pitch based carbon fibers subject to the same pretreatments................................. 89

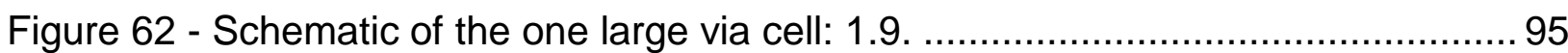

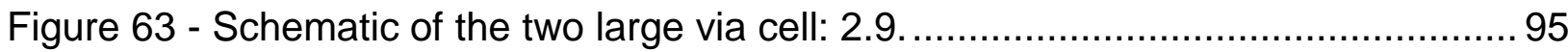

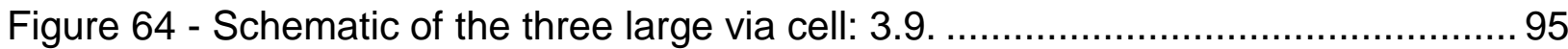

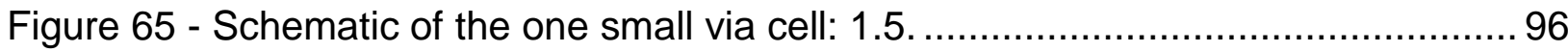

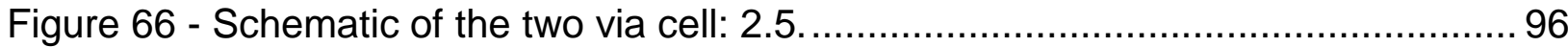

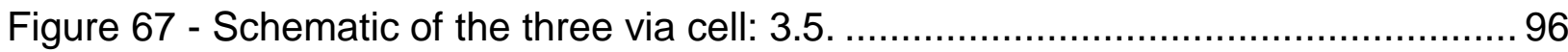

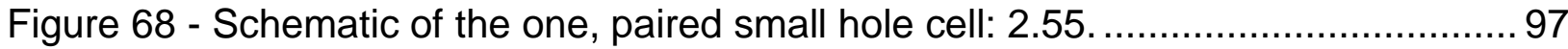

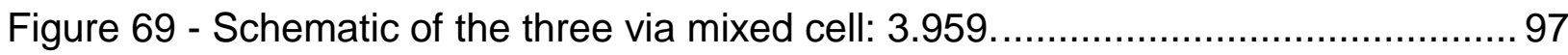

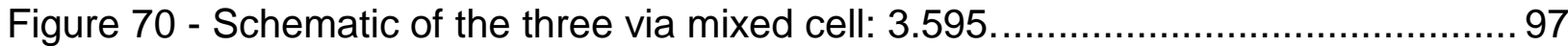

Figure 71 - Schematic of the four via diamond cell: 4.5.............................................. 98

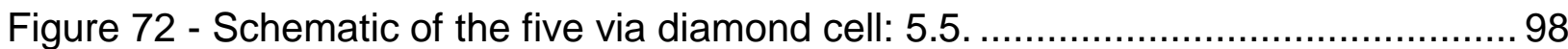

Figure 73 - Picture of applying a thin layer of paste wax, with cloth, onto the glass sheet.

Figure 74 - Schematic of typical components utilized in a vacuum forced epoxy infusion

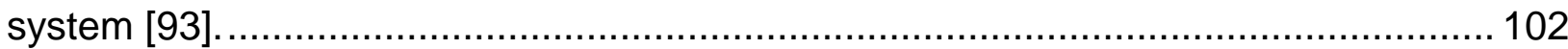

Figure 75 - Picture of in-situ epoxy flow during vacuum forced infusion on composite batteries. 103

Figure 76 - Delamination occurring in mechanical samples during four point bending. 105

Figure 77 - The stress and deflection ranges of the different proposed structural battery architectures. 106

Figure 78 - Picture of in-situ multifunctional battery analysis equipment. 109 Figure 79 - Picture of in-situ multifunctional battery composite laminate delamination, while electrochemically tested under mechanical four-point beam load. 109 Figure 80 - The in-situ analysis of a 1.9 large via multifunctional battery voltage, during a $\mathrm{C} / 2$ discharge rate, aligned with corresponding four-point beam strength evaluations.

Figure 81 - Electrochemical performance analysis of the 1.9 large via designed multifunctional battery: before, during, and after mechanical deflection investigations. 


\section{List of Tables}

Table 1 - Batch calculations for in-house synthesized $\mathrm{LiCoO}_{2}$ cathode powder............ 29

Table 2 - Batch calculations for solid state synthesized electrode inks. ...................... 41

Table 3 - Synthesized electrolyte batch calculations................................................ 46

Table 4 - Example of theoretical and experimental capacity calculations for in-house

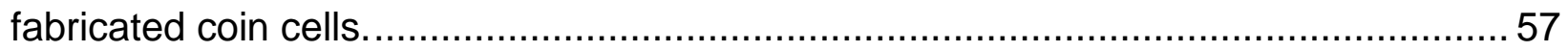

Table 5 - Tape cast electrode deposition thickness with resulting thickness of electrodes

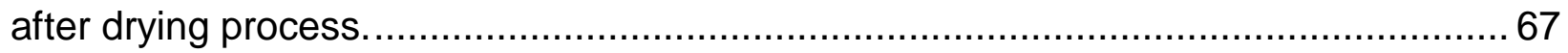

Table 6 - Capacity loss [\%] for each SEI formation, along with the observed loss through 30 cycles. 73

Table 7 - Manufacturer provided carbon fiber properties. 78 Table 8 - XPS deconvolution to C 1s peak analysis of different pretreatment altered surface functional groups on carbon fiber. 85 Table 9 - Electrochemical performance analysis of pretreated carbon fibers in respect to achieved capacity and resulting cyclic losses. 90 Table 10 - Functional area of structural battery architectures in regards to strength of architecture before flexure before delamination. 107 


\section{List of Equations}

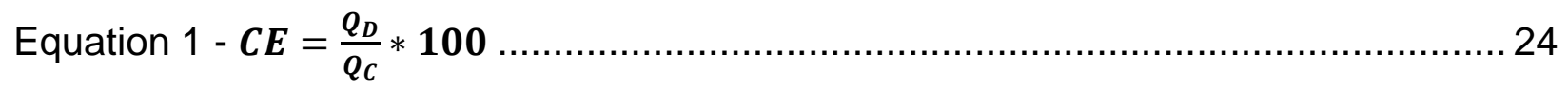

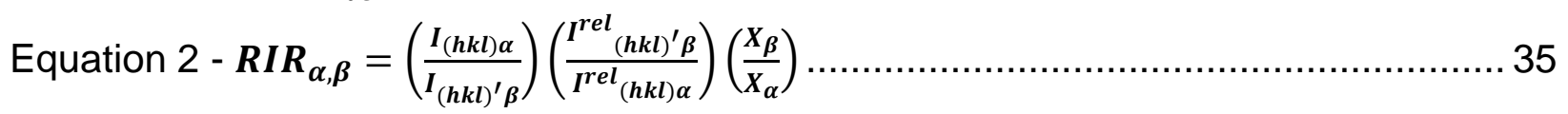

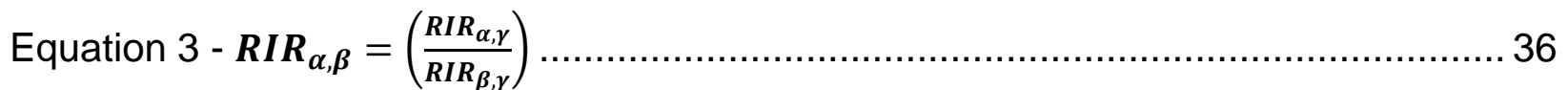

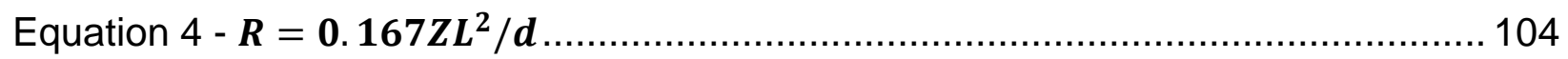

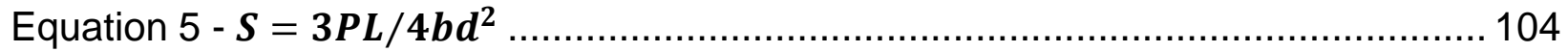




\section{Chapter 1: Introduction}

\subsection{Background}

In today's market, there is an increasing demand for more conformable lithium ion batteries with a high energy density and improved packaging design (high specific volume density) for applications in wearable electronics, transportation, and unmanned/robotic vehicles. However, in the pursuit of these battery structures, research has primarily focused on the alteration of the battery pack as a complete volumetric unit. The length, width, and thickness of the rectangular or cylindrical battery pack are typically modified to fit into a specific system, or the system is modeled after a particular battery. Another more volume efficient method of incorporating the batteries into a structure is through the interconnection of discrete, isolated batteries within structural layers of the material or component. Although this has proven to be effective, even further advancements can be made by incorporating the structure into the battery instead of the battery into the structure [1]. The aim of this alternative approach is to make the battery itself capable of bearing significant mechanical loads by changing the material composition of the battery electrodes and separator material.

\subsection{Goals of Research}

The goal of this work was to design a battery that serves as the structural material as well as the power source for a vehicle. The combination of both mechanical and electrochemical aspects within one material defines the component as a multifunctional material, or in this case, a multifunctional battery. The initial work focused on the fabrication of a novel multifunctional lithium ion battery structure, where reinforcement composite fibers were substituted and utilized as active components within the electrodes and separator, enduring cyclic voltage-capacity testing. The next part of this work investigated an approach to modify the conventional lithium ion pouch battery architecture by creating perforations (vias) through the cell architecture in order to reduce delamination or wrinkling of the battery during bending loads. The future aim is to fabricate the battery as part of the composite structure instead of a separate piece to the structure. 


\subsection{Organization}

This thesis is comprised of six chapters. The first chapter presents an introduction of the topic, as well as the demand in the current and future market. The second chapter presents a literature review to provide the concepts required for understanding the intricate architecture of a lithium ion battery, why it is chosen, how other organizations have approached the concept of a multifunctional battery thus far, and the aspirations of this research.

The third chapter is composed of the processing techniques for fabricating a classical lithium ion single cell battery. It goes through each process required for the replication of this type of cell, from the synthesis of the powder to the construction of the battery. The testing protocol and baseline performance data of these cells are included in this section.

The fourth chapter focuses on the multifunctional material, carbon fiber, and how it can be utilized as an anode in the lithium ion battery chemistry. Several pretreatments with the objective of maximizing the cyclic ability of these fibers were performed and the resulting performance data is included.

The fifth chapter illustrates how a classical lithium ion cell can be modified for object specific applications. Various mock cells were constructed and tested through a traditional four-point bending method for strength and flexure measurements. This data is used to design and fabricate the intricate battery architectures with a specific shape to fulfill the functional battery requirement to conform to the proposed vehicle structure.

Lastly, the sixth and final chapter provides a summary of the conclusions made in each chapter of the collected data and continues with a further discussion of the results. This chapter also elaborates on the future work required in this area to sufficiently produce truly multifunctional batteries. 


\section{Chapter 2: Literature Review}

\subsection{General Description of a Battery}

The battery is an electrochemical device that converts chemical energy that is stored in its active materials directly into electrical energy through oxidation/reduction processes. A typical battery is made up of multiple single electrochemical cells, which are connected together in either series (for higher voltage) or parallel (for a higher capacity) [2]. These connected cells create a static potential for power and release of the stored electrical charge when connected to a load, such as a circuit or motor [3].

\subsection{Components of a Battery}

A typical lithium ion battery consists of three main distinguishable components which are the anode, cathode and, electrolyte/separator material. The anode serves as the negative electrode and releases electrons to the external circuit during the electrochemical oxidation reaction; similarly, the cathode serves as the positive electrode which accepts electrons from the external circuit and is reduced during the electrochemical reaction [3]. The electrolyte is a liquid mixture, such as water or solvent mixed with salts, acids, or alkalis to aid in ionic conductivity, or a solid ionic conductor. The separator material along with the electrolyte provides a medium for the transfer of ions, while resisting electrons, inside of the cell between the anode and the cathode [2].

\subsection{Categories of Batteries}

Batteries are classified into three general categories, each type with advantages and disadvantages. These categories are primary (non-rechargeable), secondary (rechargeable) and reserve (non-active). Each one of these categories is further explained to identify their specific electrochemical architecture [2].

\subsubsection{Primary Battery}

Primary batteries can produce current immediately after assembly; however, they are not capable of easily or efficiently being recharged, since the chemical reactions are not easily reversible and the active materials may not return to their original forms. 
Primary batteries are also known as disposable batteries, since they are intended to be used once and discarded.

They have a good shelf life, high energy density at low to moderate discharge rates and are easy to use; additionally, primary batteries are convenient, inexpensive, and lightweight. These batteries are commonly used in portable electronic devices that are only used occasionally, or when away from an alternative power source, such as alarm and communication circuits where other electric power is intermittently available [2], [3].

\subsubsection{Secondary Battery}

A secondary battery must be charged before use; they are usually assembled with active materials in the discharged state. Rechargeable batteries or secondary cells are storage devices for electrical energy and are also known as storage batteries. The substantial difference between these batteries and primary batteries is that they can be recharged to their initial condition by applying electrical current in the opposite direction of the discharge current with a cell charger, which reverses the chemical reactions that occur during use.

Secondary batteries have desirable attributes such as a high power density, high discharge rate, flat discharge curves, and effective low temperature performance. The oldest form of a rechargeable battery is the lead acid battery which is utilized in automotive vehicles. Other uses for these cells are in aircrafts, emergency power sources, hybrid electric vehicles, stationary energy storage, portable consumer electronics, and power tools [2], [3], [4].

\subsubsection{Reserve Battery}

A reserve battery, also called a stand-by battery, is a primary battery where a component is isolated keeping it inert until it is activated for use. When long storage is required, reserve batteries are used, since the active chemicals of the cell remain segregated until needed, thus reducing a self-discharge effect. 
These batteries can be activated by various methods, depending upon their chemistry. Some methods for activating specific forms of these batteries are as follows: by the addition of water, by adding an electrolyte, by introducing a gas into the cell that is either the active cathode material or part of the electrolyte, or by heating a solid electrolyte to a temperature at which it becomes conductive. Reserve batteries are typically used in military applications, such as missiles, because of their potential long shelf-life, higher energy density, and relatively short usage time after activation [2], [3].

\subsection{Lithium Battery History}

In past decades, the dominant battery systems have been the primary-type battery based on manganese dioxide/zinc chemistry and the secondary-type battery based lead acid chemistry; both at this time are at an advanced stage in their development. However, in today's market lithium ion batteries are challenging these traditional systems as the dominate choice for high performance battery systems [2]. The development of this system has taken several decades thus far, and new breakthroughs within the cells components are steadily improving the high energy possibilities of this system. The basic reason that lithium ion batteries are challenging these tradition systems is the superior energy density possible due to lithium's low atomic number and high electrode potential which can create a high energy density. A comparison of battery specific energy and energy densities are shown in Figure 1 [2], [3]. 


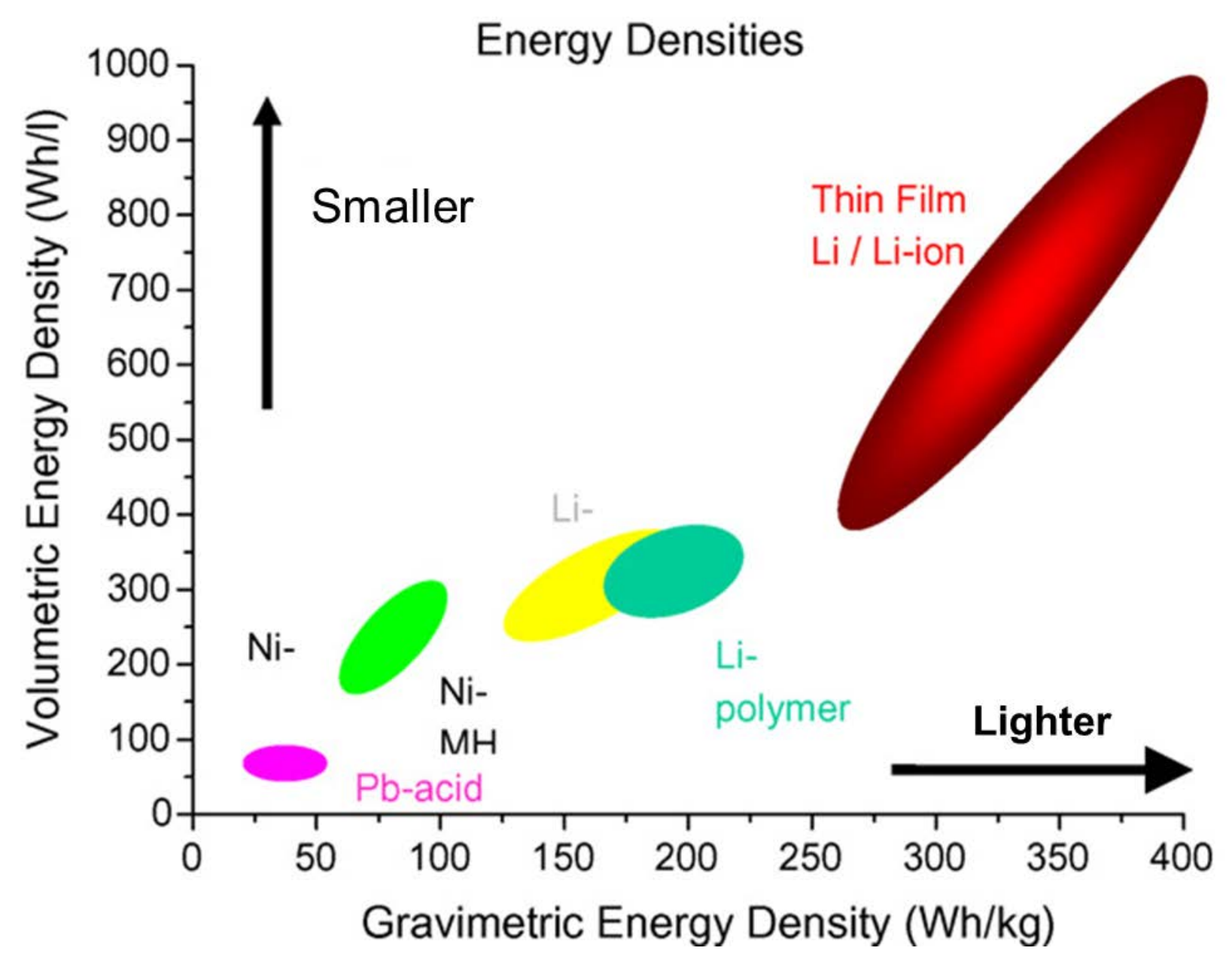

Figure 1 - Comparison of the volumetric and gravimetric energy density with different battery systems [5].

Lithium metal anode batteries were first established in the early 1970's; however, these systems were not suitable for secondary batteries due to dendrites forming through the cell causing shorting and safety issues [3]. It took several more years with numerous efforts of different cell component configurations until the early 1990's when a commercially available lithium ion rechargeable battery was developed and put on the market by Sony. This is marked as the birth of the current lithium ion battery [5]. These early cells contained a lithium cobalt oxide as one electrode and a carbonaceous material as the counter electrode. Although no lithium metal is in these cells, technically a lithium polymer type battery, the name lithium ion is accepted by the battery community for this type of cell [6]. 


\subsection{Lithium Battery Architecture}

Battery architectures have come a long way since the first practical invention in the $18^{\text {th }}$ century when batteries were contained in glass jars [4]. The commercialization of batteries has led to the design architecture of lithium ion batteries to be classified into four different configurations. These architectures are the cylindrical cell, button cell, prismatic cell, and the pouch cell [2].

The cylindrical cell (Figure 2) is the most common style for both primary and secondary batteries; this is due to the ease of manufacturing and dependent mechanical stability. The most common type of this cell is the 18650 lithium ion cell and this cell is typically used as battery packs in hand tools and laptops. The electrodes in this cell are prepared as thin strips, then rolled within the separator and placed in the cylinder. These cells have a high surface area, but are not the most efficiently packed design [2], [7], [8].

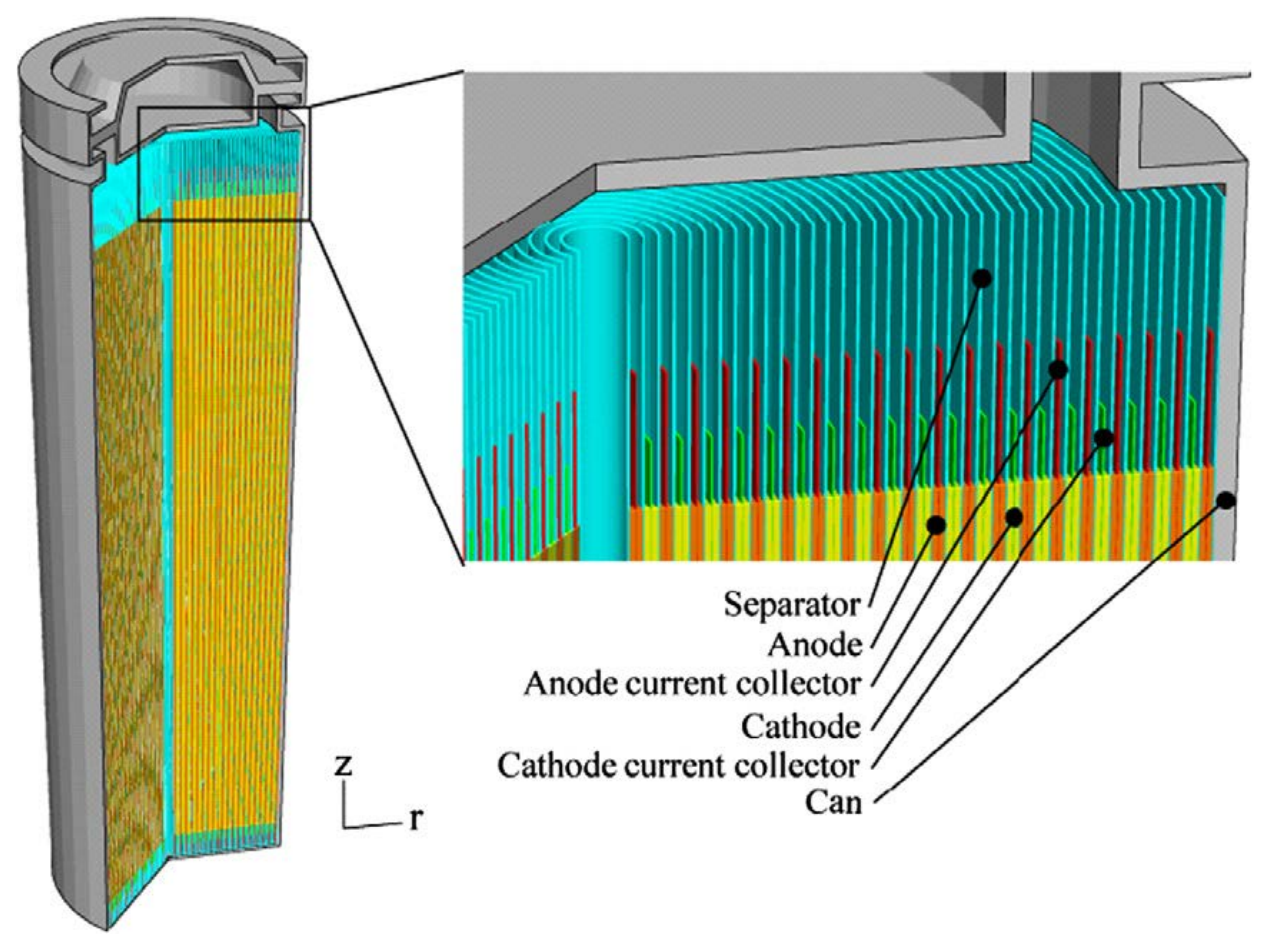

Figure 2 - Diagram of a classical spun cylindrical cell type battery construction [8]. 
The prismatic cell and the pouch cell are both very similar in design and are wrapped for packaging in the same way. The main differences are the packaging of the cell and the materials of the electrodes.

The prismatic cell's walls are welded aluminum housings encased in plastic, which directly determines the mechanical strength of the cell. Although they are more efficient than the cylindrical cells, the design is costly to manufacture (Figure 3). Furthermore, these cells have no general format, which causes each manufacturer to design their product to fit around a specific producer's cell. These batteries are predominately found in cell phones and small portable electronics [2], [9].

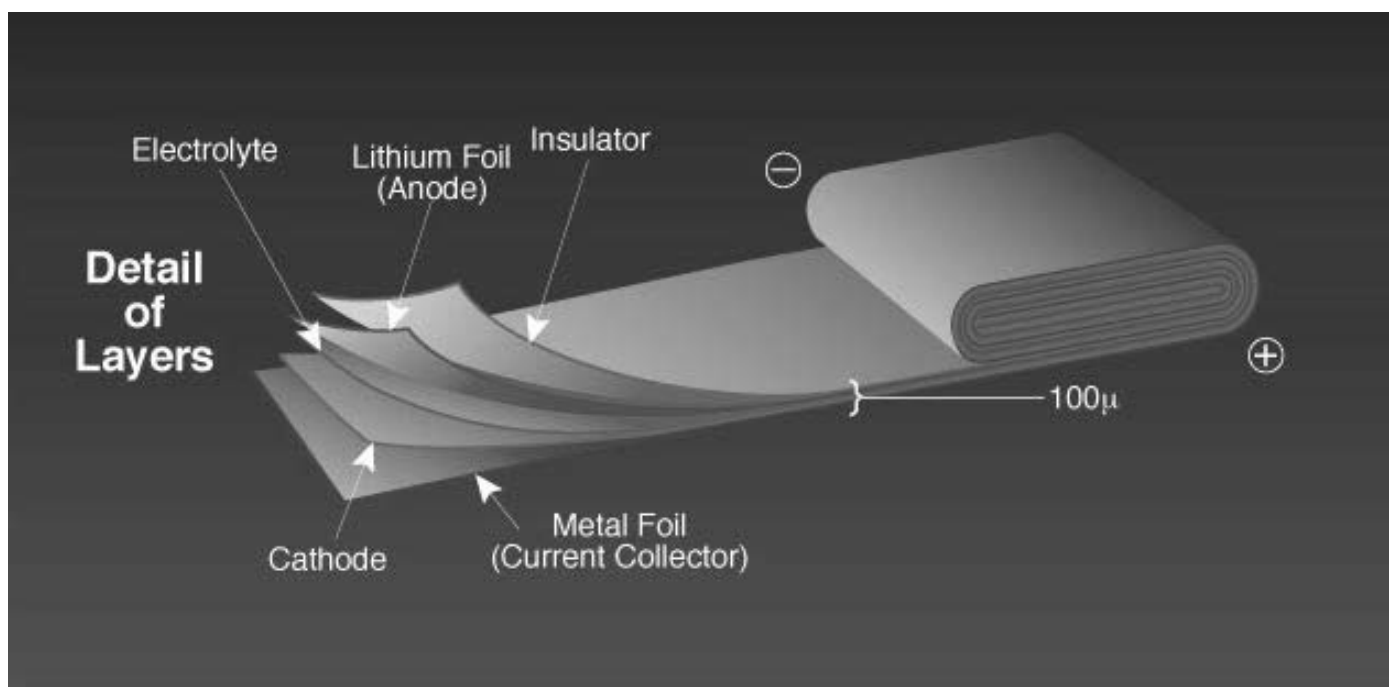

Figure 3 - Laminated lithium prismatic cell layers [2].

The pouch cells differ in which the electrodes must contain structural polymer materials in order to compensate for the decrease in mechanical properties of the package, but this design achieves the highest efficiency of use of all materials (Figure 4). The casing of these cells are simply placed within an aluminum polymer coated bag, which leads to safety issues such as swelling from high usage and being able to be punctured easily (Figure 5). These cells can be designed to fit specific applications, but there are only a few companies willing to fabricate customized cells and small lots of them can cost thousands of dollars [1], [2], [10]. 


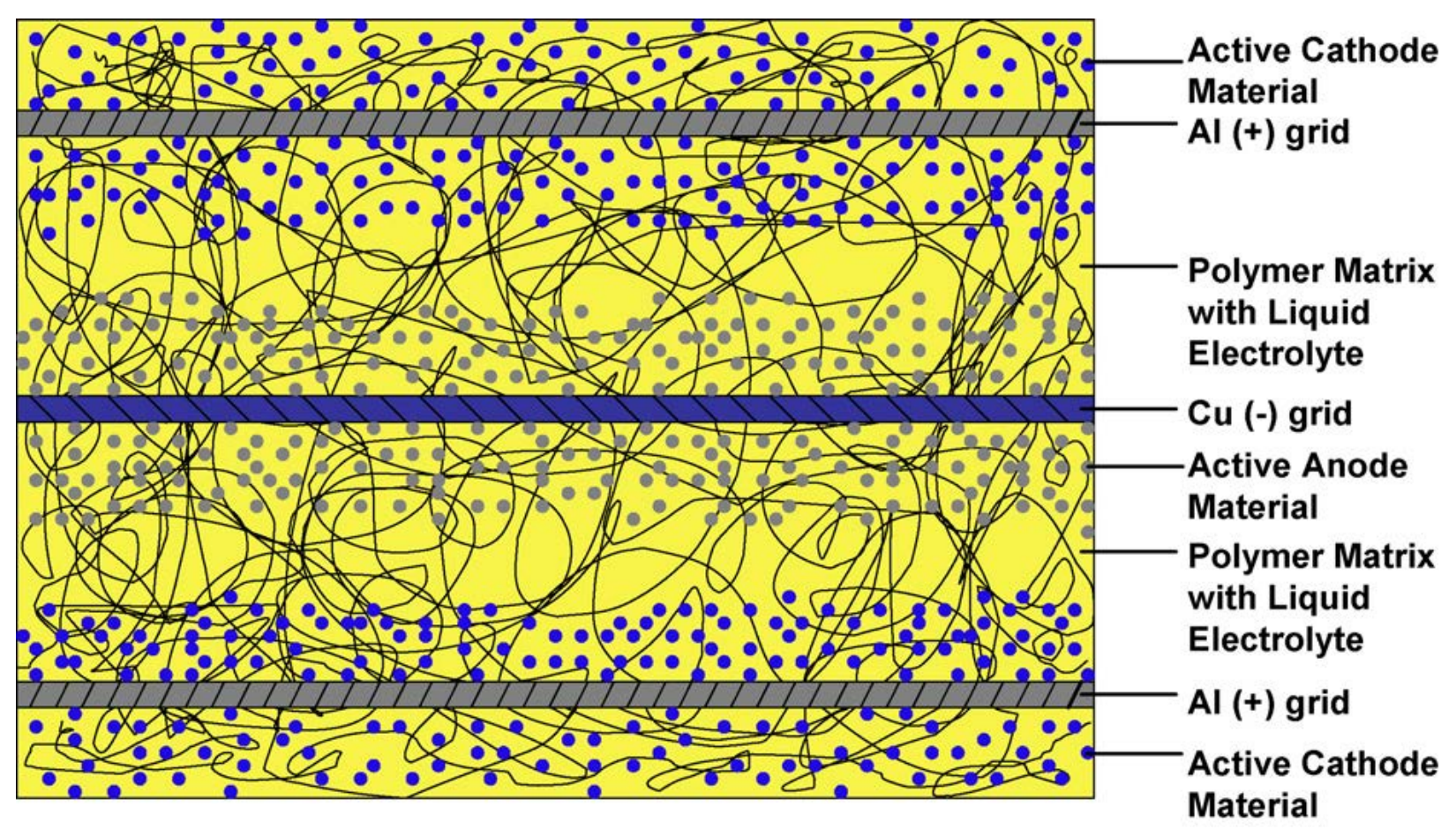

Figure 4 - Illustration of polymer enhanced electrodes in a pouch battery [11].

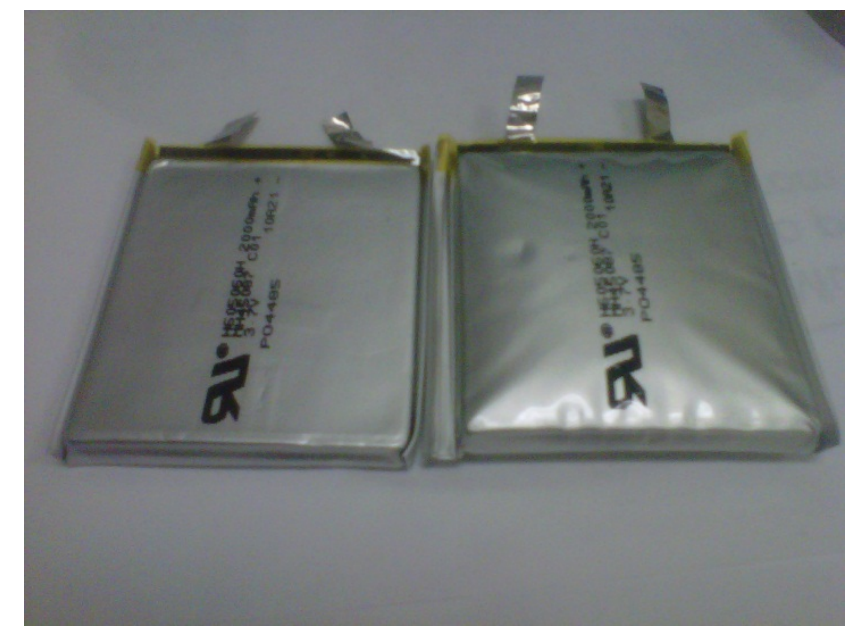

Figure 5 - Demonstration of the swelling effect in pouch batteries.

The coin cell is the smallest and most inexpensive type of battery to fabricate (Figure 6). These cells are offered in several sizes and are extremely durable. Cells contain only one layer making it a true single cell battery, although they are commonly 
stacked in a series to accumulate a high voltage. Uses for these cells are limited to low current demanding electronic devices such as clocks, watches, and hearing aids [2], [4], [9].

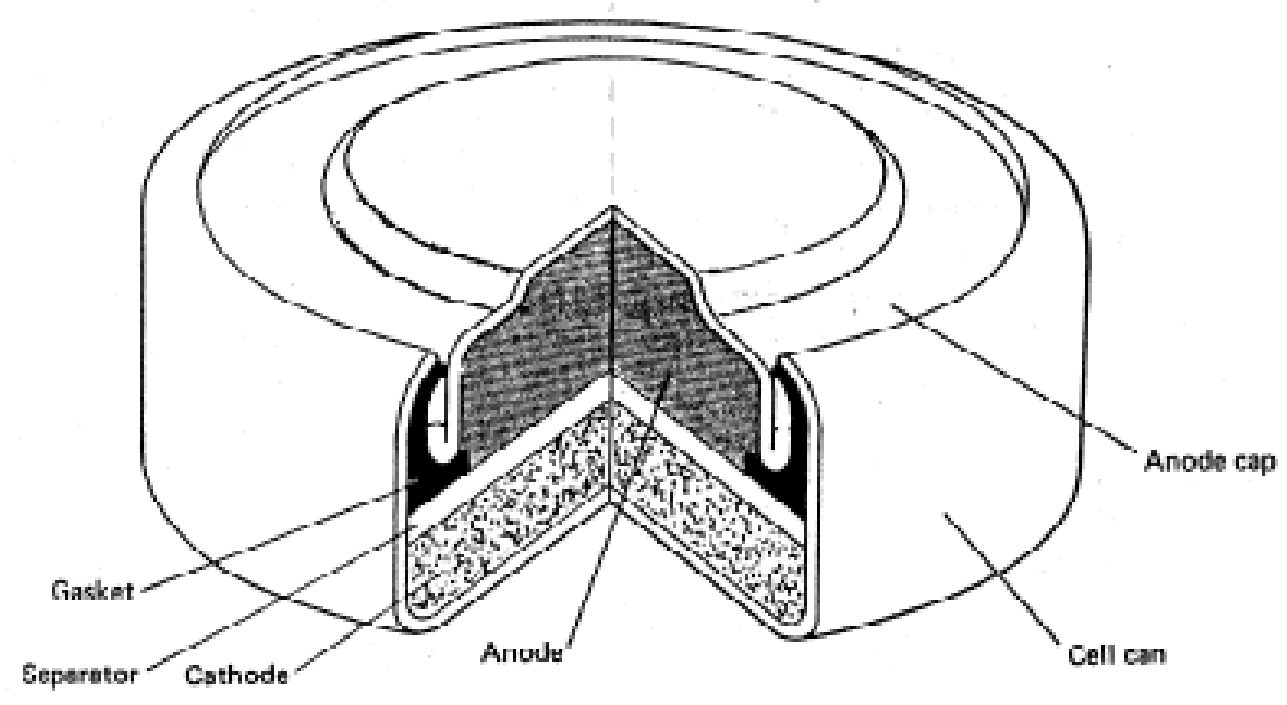

Figure 6 - Cross sectional view of common layers in a coin cell [4].

In summary of the advantages and disadvantages of these cells, the cylindrical cell is very dependable, but has a low package density. Prismatic cells have an improved package design compared to the cylindrical, but are more expensive to manufacture. Pouch cells take the most advantage of their packaging and are cheaper to manufacture, but swelling can ruin the cell as well as cause safety issues. Coin cells are the smallest and cheapest available cell to produce, however their applications are limited and are not suitable for high powered applications.

\subsection{Battery Manufacturing Process}

The process for manufacturing lithium batteries is the same for most styles of cells, regardless of materials; this similarity spans all the way until structural encasing of the actual battery. The basic steps of manufacturing are as follows: 1) synthesizing the active electrode into slurries and casting them onto thin metal foils, 2) winding the two electrodes with a separator between them and cutting them to size, 3) inserting the wound portion into a case, 4) adding the electrolyte to the cell, and 5) sealing the case 
(Figure 7). The only difference is that coin cells are unrolled and cut before insertion into the cell. Further manufacturing steps are discussed in this section [3], [7].

Battery Manufacturing Process: Electrode Compound Preparation to Winding.

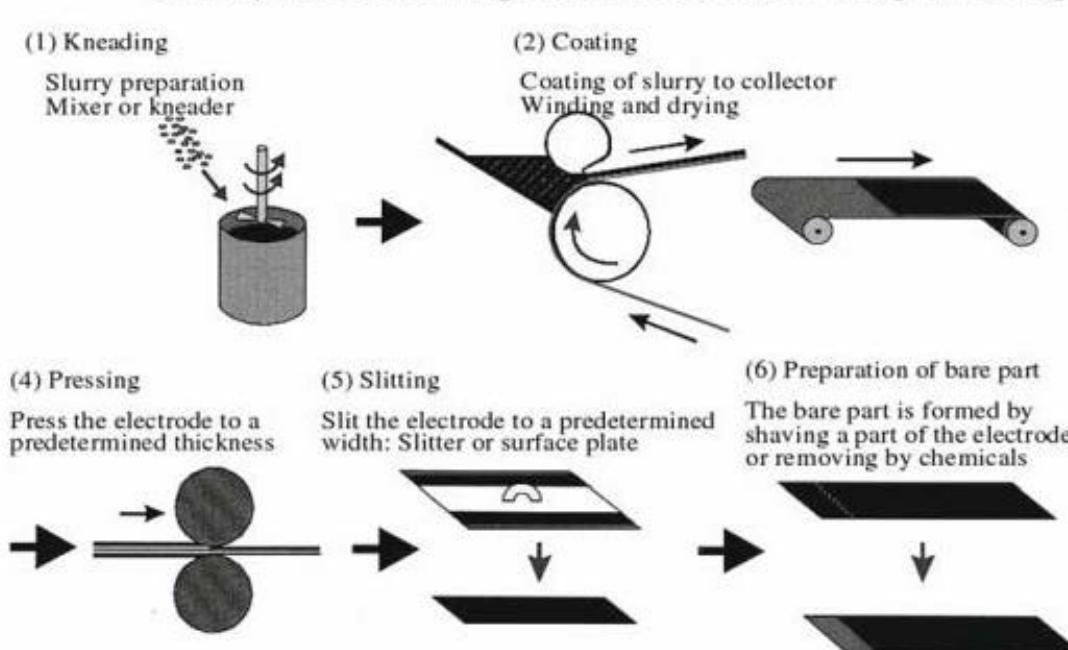

(3) Cutting of electrode

(1) Kneading

(2) Coating

Cut to predetermined size

Coating of slurry to collector

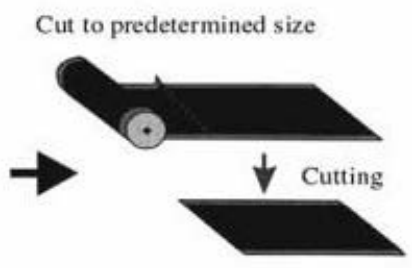

(7) Terminal mounting

The terminal is welded to the bare part.

Ultrasonic welding machine or

Resistance welding machine

(8) Winding

Ultrasonic,

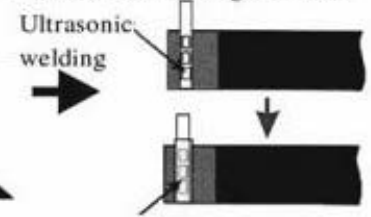

Tape is affixed

The electrodes are wound flat by placing the

separator between them by a winding machine

Positive electrode Separator Negative electrode
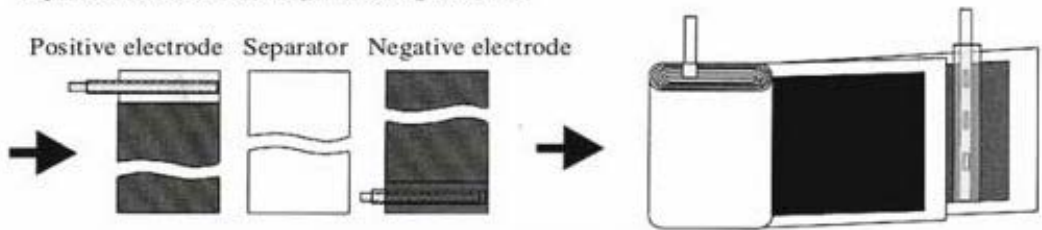

(9) Completion

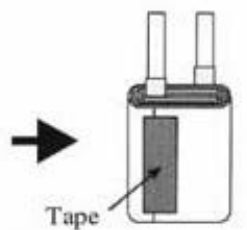

Figure 7 - General overview of the several processes involved in manufacturing lithium batteries [3].

\subsubsection{Powder Synthesis}

There are several techniques used for the synthesis of the active electrode materials such as solid state reactions, hydrothermal synthesis, and the sol gel method. Although these materials can be commercially purchased, companies generally synthesize in-house powders through these techniques [6].

The standard procedure for producing active electrode materials has been through a solid state reaction process. For $\mathrm{LiCoO}_{2}$, this reaction contains hydroxides and carbonates such as $\mathrm{LiOH} \bullet \mathrm{H}_{2} \mathrm{O}, \mathrm{Li}_{2} \mathrm{CO}_{3}$ and $\mathrm{CoCO}_{3}$, which are milled for several hours. Milling is followed by a calcination heat treatment process at $850-900^{\circ} \mathrm{C}$ for prolonged periods to complete a phase transition in the materials. Once calcined, the 
particles are milled longer to achieve a smaller particle size. This technique results in larger particle sizes, higher calcination temperatures, and an irregular morphology compared to the other techniques (Figure 8) [12], [13].

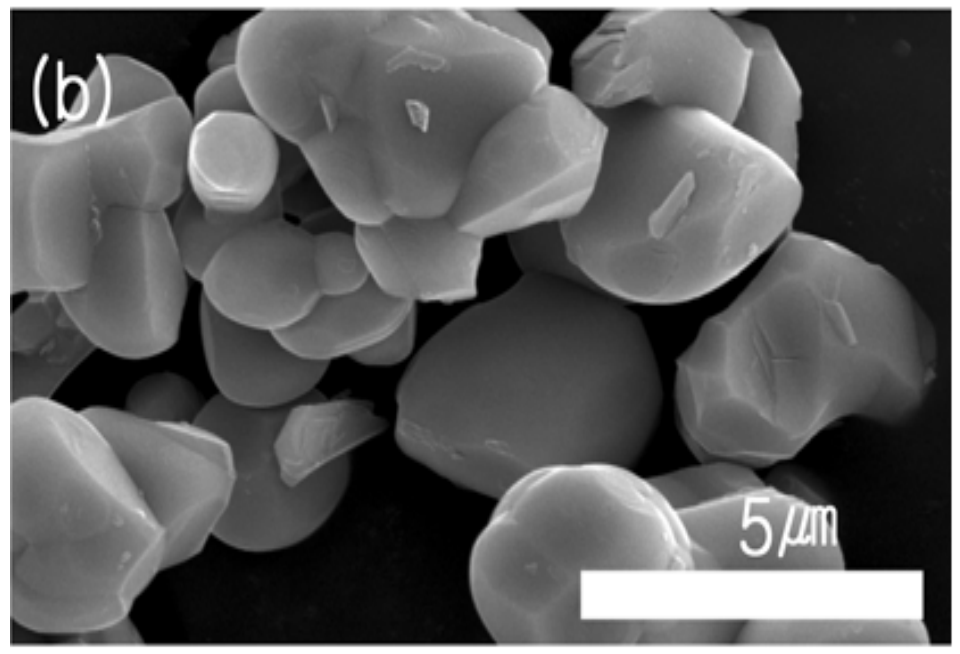

Figure 8 - SEM micrograph of large grain powders made from a classical solid state synthesis [13].

The hydrothermal process is a method for producing nano-sized powder from an elevated temperature and pressure. Precursor powders are added together slowly into an aqueous solution. The solution is then stirred at a higher temperature in a pressure vessel, such as an autoclave, for the reaction process. This process produces ultrafine pure particles at lower temperatures (Figure 9) [14].

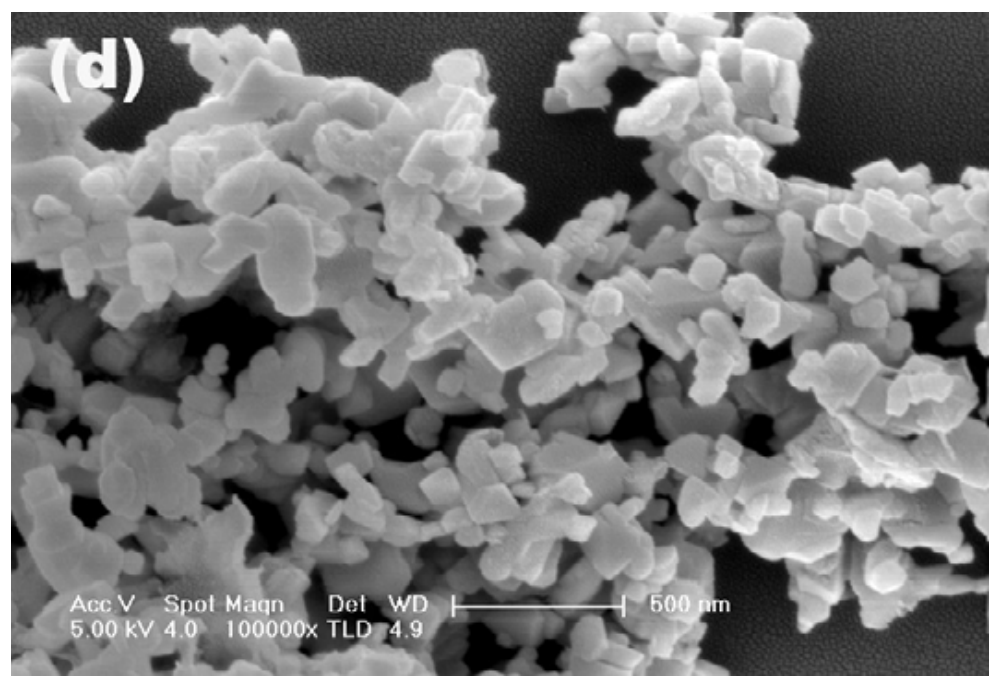

Figure 9 - SEM micrograph of $\mathrm{LiCoO}_{2}$ powders obtained from a hydrothermal synthesis [14]. 
The sol gel method is another method for producing ultrafine powder particles from a wet chemical technique. This technique takes aqueous solutions and mixes them together while controlling the $\mathrm{pH}$ of the solution until a gel precipitate is formed. The precipitate then forms a precursor powder which is calcined during a thermal decomposition process. This synthesis forms highly homogenous particles in a quick process and at lower temperatures (Figure 10) [12], [13], [15].

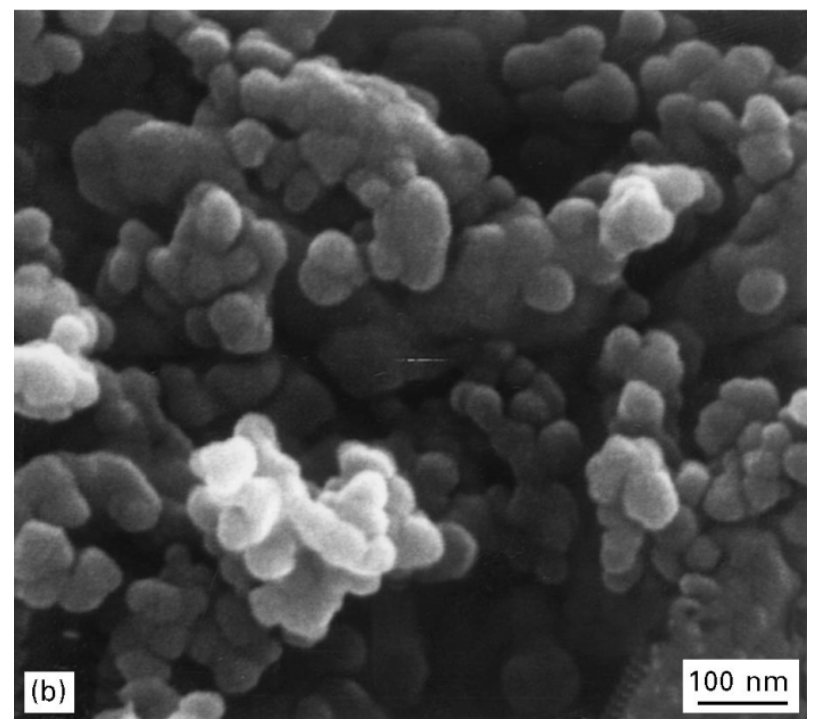

Figure 10 - SEM micrograph of $\mathrm{LiCoO}_{2}$ powders derived from a sol gel method [12].

\subsubsection{Ink synthesis}

The cathode is generally composed of $\mathrm{LiCoO}_{2}$ powder, a conductive additive, and a polymer binder to help hold everything in the electrode together. A conductive additive is required due to the poor electronic conductivity $10^{-9} \mathrm{~S} / \mathrm{cm}$ of $\mathrm{LiCoO}_{2}$ at room temperature [16]. These materials must be homogeneously mixed together with a solvent before proceeding to the deposition process. The solvent content should be as low as possible leaving a high particle load in the inks to avoid any defects during the drying process after deposition. However, the viscosity of the slurries must be low enough in order to ensure a homogenous mass flowing during the deposition process [17]. Processing of the electrodes is classified by the type of solvent used: water based or aqueous solution, and organic based or non-aqueous slurry. Water based processing is preferred in large-scale operations due to the environment and cost, but agglomeration of particles are a severe problem and will lead to poorly performing 
electrodes. Furthermore, the challenge of removing all of the water content remaining in the electrodes prior to construction of the batteries has proven problematic. Although solutions to these problems are being researched, a clear cost effective solution has not been evident enough for implementation away from organic solvent based systems [18].

Density of the electrodes is one of the biggest factors that manipulates the electrodes properties and is made up of both sheet density and packing density. When even a $1 \%$ increase of electro-active material is added, which increases the density of the cell, a substantial improvement of cell capacity is evident [3].

In typical non-aqueous solutions with $\mathrm{LiCoO}_{2}$ powder, the conductive additive is a type of carbon black and a polymer binder of polyvinylidene fluoride (PVDF). Usually these materials are mixed together by sonication or solid state synthesis means in a stable suspension of $\mathrm{N}$-methyl-2-pyrrolidone (NMP) as the solvent. Almost every research group uses a different electrode composition with $\mathrm{LiCoO}_{2}+$ carbon black + PVDF, but all in the range of (80/10/10 wt\%) to (95/2.5/2.5 wt\%). The counter electrode is made via the same process by substituting graphite for the cathode powder and removing the conductive additive of carbon black, since it is not electrochemically required. The amount of solvent used in these inks was dependent upon the materials and the electrode deposition process [6], [11].

\subsubsection{Electrode Deposition}

There are several techniques used for depositing the electrodes onto conductive substrates, which are spin coating, screenprinting, and tapecasting. The conductive substrates are aluminum foil for the positive electrode and copper foil for the negative electrode. These foils are selected due to their mechanical strength, thinness, cost, stability in the electrolyte mixture, and adhesiveness of inks to the foils [6], [15], [19]. The deposition processes are different in their own respects and range from electrode thicknesses of 10-300 $\mu \mathrm{m}$ [20], [21] . The particle size from nano- to micro-size utilized throughout the electrodes directly correlate to the diffusion of lithium ions throughout the

electrode during cycling. The drying process of inks on the substrates are relatively the same, in which once deposited they are dried under vacuum from $80-120^{\circ} \mathrm{C}$ for at least 8 hours. An evaluation of the comparative electrochemical performance from each 
deposition method, as a function of drying time involved, and ink formulation has not been completed [6], [11], [22].

\subsubsection{Cell Construction}

To construct a battery the next step is to cut the electrodes and separator to fit into the type of cell for assembly, stack or wind of the components, and fit them into the cell. The procedures are required to be completed in an inert atmosphere such as a glove box or a dry room due to the electrolyte's extreme sensitivity to moisture. Once under inert atmosphere, the electrolyte is injected into the cell where time is allowed for the electrolyte to permeate completely throughout the electrode before sealing. After the battery is sealed, it is ready to undergo electrochemical testing [7], [23].

\subsection{Lithium Battery Chemistry}

The chemistry and characteristics of a lithium ion battery correlate directly with the cell's components. Selection of these components are done together in order to achieve an optimum balance between each part in order to obtain a desirable battery which has a high energy density, high power density, long life cycle, as well as a high degree of safety based on the desired application. A chart illustrating a variety of materials that have been utilized in the fabrication of lithium ion cells are shown in Figure 11 [2]. 


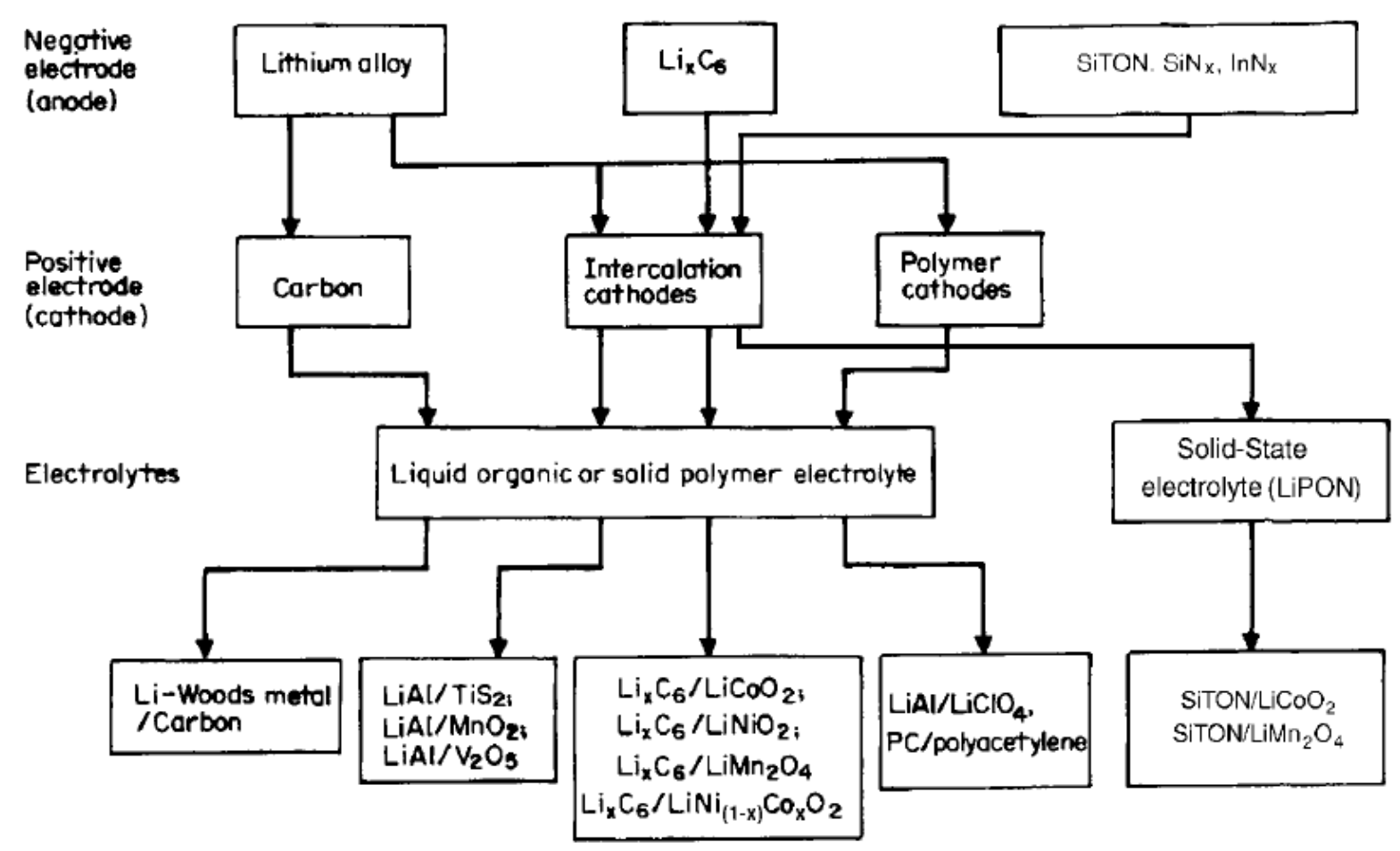

Figure 11 - Variety of secondary type lithium ion battery components [2].

This section only discusses the chemistry involved with the traditional secondary type lithium ion battery with components consisting of a lithium cobalt oxide $\left(\mathrm{LiCoO}_{2}\right)$ cathode, carbonaceous (graphite) anode, and a lithium salt carbonate electrolyte. In this configuration, graphite serves as the negative electrode and has the purpose of giving up electrons to the external circuit and is oxidized during the electrochemical reaction; similarly, $\mathrm{LiCoO}_{2}$ serves as the positive electrode which accepts electrons from the external circuit and is reduced during the electrochemical reaction. The electrolyte is a liquid gel mixture that aids in conductivity and connects through a porous polymer separator material. These materials provide a medium for the transfer of ions, while resisting electrons, inside of the cell between the anode and the cathode [2]. A further explanation of this process is that both the anode and cathode are materials that lithium ions migrate in and out of their structure. During the charging process, lithium ions undergo intercalation (insertion) within the graphite when the ions move into the anode. During the discharge process de-intercalation (extraction) occurs in which lithium ions moves back out of the graphite and returns to the cathode. A diagram of the chemistry 
behind this phenomenon and illustration of how a battery charges and discharges is shown in Figure 12 [24], [25].

\section{Lithium Ion Battery}
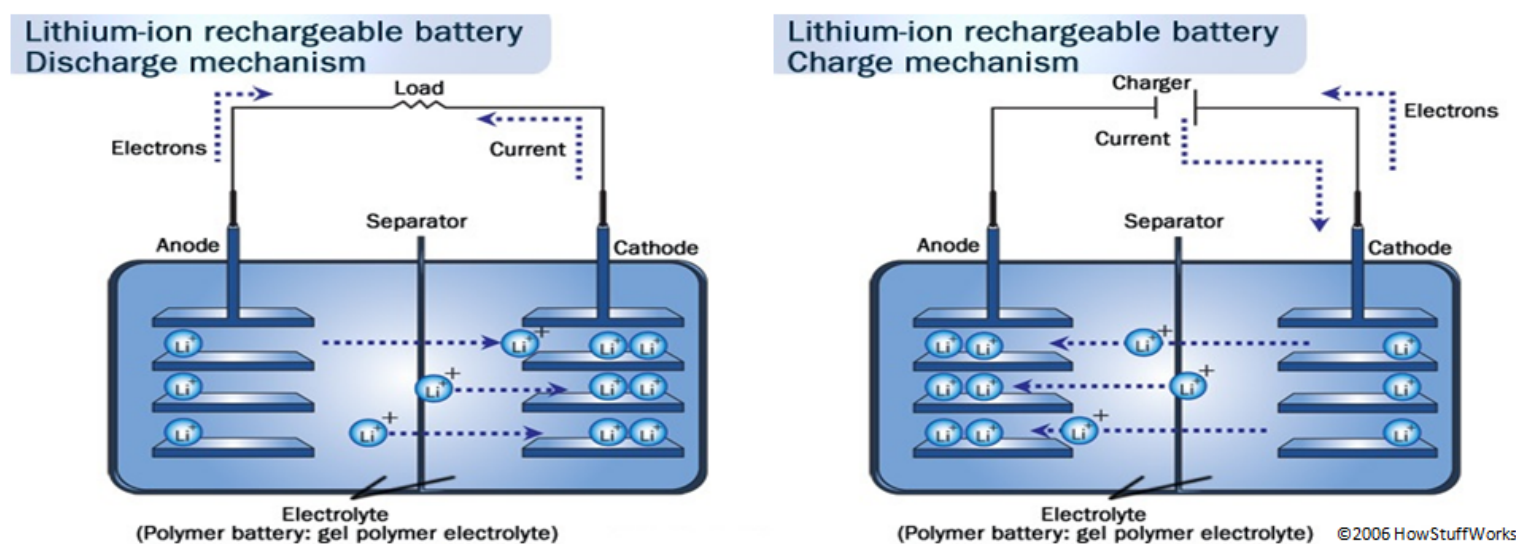

\section{Lithium Cobaltite Half Reaction \\ Cathode: $\mathrm{LiCOO}_{2} \leftrightarrow \mathrm{Li}_{1-x} \mathrm{CoO}_{2}+x \mathrm{Li}^{+}+x e^{-}$ \\ Anode: $x L^{+}+x e^{-}+6 C \leftrightarrow L i_{x} C_{6}$}

Figure 12 - Charge and discharge chemistry of a typical lithium ion battery [24].

\subsubsection{Cathode}

The most commercialized cathode material used in rechargeable lithium ion batteries is lithium cobalt oxide $\left(\mathrm{LiCoO}_{2}\right)$ which holds over $90 \%$ of the market. The cathode material determines the voltage, capacity, and specific energy of the cell, while the cycle life and capacity fade is dependent upon the interaction between the other material interfaces in the cell. This material has a layered rhombohedral structure (Figure 13) which contains alternating planes of lithium ions and cobalt (III) separated by cubic close packed arrays of oxygen ion layers which enables two dimensional diffusion of the lithium ion [26], [27]. Even though $\mathrm{LiCoO}_{2}$ has been used for decades, advances in other areas such as pulse charging techniques and doping with other highly ionic materials of aluminum and magnesium has given this already corner stone cathode material vast improvements in cyclic ability and capacity [3]. 


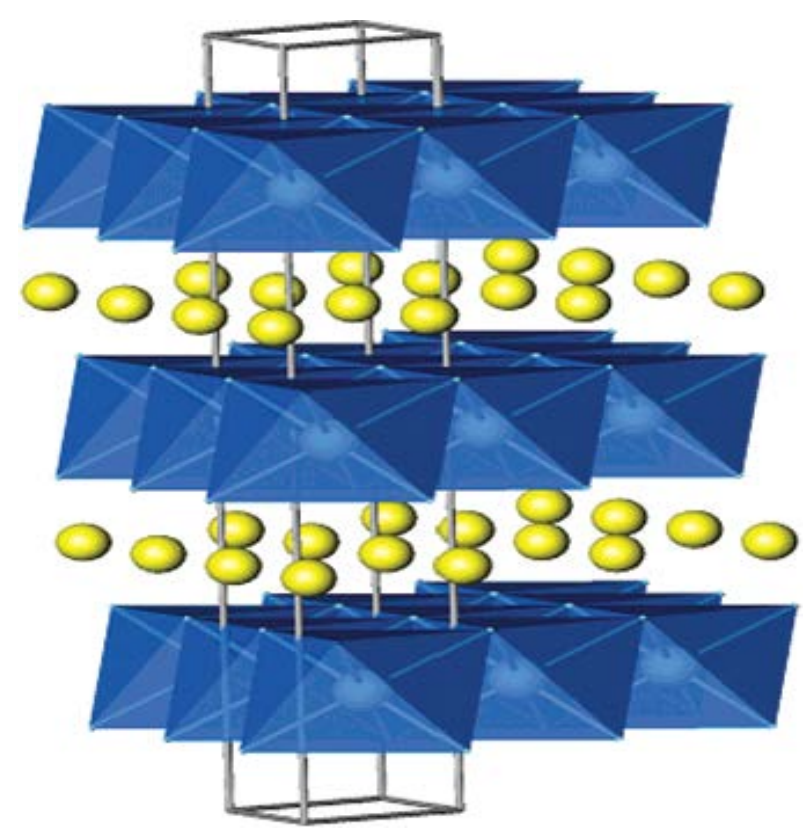

Figure 13 - Layered structure of $\mathrm{LiCoO}_{2}$ showing the lithium ions between the transitional metal oxide sheets [25].

The theoretical capacity for $\mathrm{LiCoO}_{2}$ is $272 \mathrm{mAh} / \mathrm{g}$, although the reversible capacity is limited to about $140 \mathrm{mAh} / \mathrm{g}$ when cycled safely between 3.0 and $4.2 \mathrm{~V}$ [11], [28]. $\mathrm{LiCoO}_{2}$ has a large highly reversible voltage range from 2.75-4.3 $\mathrm{V}$, although through every cycle a gradual irreversible loss in capacity and power occurs. The actual capacity of the cell is primarily determined by the specific surface area of the electrodes. A high surface area in a cell leads to an improved rate performance and higher capacity. The density of the material inevitably decreases when the surface area increases, so there are limits to the specific surface area. If only nanoparticles are used, the performance may be initially improved, but the cycling and safety of the cell are extremely decreased leading to high possibilities of overheating and combustion. The desirable average particle size and distribution are different for each manufacturer depending on the specific cells they are producing, but are generally between 1-8 $\mu \mathrm{m}$ [3], [6], [15], [29], [30].

\subsubsection{Anode}

The anode in most secondary lithium ion batteries is graphite due to its high theoretical capacity of $370 \mathrm{mAh} / \mathrm{g}$ creating the $\mathrm{LiC}_{6}$ compound, one lithium ion for every six carbon atoms during lithium intercalation, even though other materials have higher 
theoretical capacities such as silicon at 4,199 mAh/g [31], [32]. Carbonaceous anodes are the most utilized anode material due to their low cost, availability, and the fact that other anode materials experience poor cycling capabilities with a high irreversible capacity loss [33], [34]. The average particle size for graphite ranges from 2-40 $\mu \mathrm{m}$, depending on the rate intended for the battery to supply [35]. Smaller particle sizes may also function as the anode; however, during the intercalation and deintercalation processes the volume of the graphite particles change. During this constant change of volume the outer surfaces of the particles begin to break down losing electrical contact which accumulates and leads to an irreversible loss of capacity. Furthermore, this loss involves electrolyte decomposition and formation of a solid electrolyte interface (SEI) layer on the anodes surface, which is further discussed in battery cycling (Section 2.8) [36].

\subsubsection{Electrolyte}

The purpose of the electrolyte is to act as an ionic conductor with the role of transferring lithium ions to and from the anode and cathode as the battery is charged and discharged. This is done by the electrolyte seeping throughout the electrodes and transferring ions smoothly at the interface between the liquid electrolyte and solid particles [3]. Conventional electrolytes consist of lithium salts in organic solvents, salts being lithium perchlorate $\left(\mathrm{LiClO}_{4}\right)$, lithium tetrafluoroborate $\left(\mathrm{LiBF}_{4}\right)$, or lithium hexafluorophosphate $\left(\mathrm{LiPF}_{6}\right)$, with $\mathrm{LiPF}_{6}$ being the preferred salt due to its rapid dissolution into the solvents and low cost. The organic solvents used are a mixture of cyclic carbonates such as ethylene carbonate (EC), or propylene carbonate (PC) and linear carbonates of dimethyl carbonate (DMC), ethyl methyl carbonate (EMC), or diethyl carbonate (DC). Cyclic carbonates have a higher viscosity but are essential in forming a stable SEI film on the anode, while linear carbonates are used to lower the viscosity of cyclic carbonates and improve the lithium ion transportation throughout the cell [3], [6], [37].

Propylene carbonate is an excellent cyclic carbonate due to its high dielectric constant, wide temperature range, and compatibility with the other components. However, PC either reacts with the graphite in the electrode and does not allow the 
battery to charge, or it creates a plateau at a lower voltage than fully charged, or it does not form an SEI layer due to the PC intercalating with lithium ions into the graphite during charging resulting in a continuous decomposition and severe exfoliation of the graphite layers until the electrode is fractured [6], [38], [39]. Typical electrolyte systems contain a mixture of $1 \mathrm{M}$ lithium salt and any type of mixture of the carbonate solutions containing both cyclic and linear carbonates. There are safety issues with these solvents due to their high flammability and harmful vapors, which leads to additives of flame retardants to increase the safety of these cells [6].

\subsubsection{Separator}

A separator has the essential function of preventing physical contact of the positive and negative electrodes, but it does not actively participate in the cell's reactions. There are several requirements for separators since their physical makeup does affect the cell's performance. The separator material must be chemically stable within the harsh environment of a lithium ion cell by not degrading over time or use, and at the same time have a high wettability by absorbing and retaining the electrolyte permanently. Furthermore, a low thickness of about $25.4 \mu \mathrm{m}$ is desired for high energy density batteries, but a compromise must be made to maintain mechanical strength of the separator for maintained safety. Another important aspect is the porosity and pore size, a typical porosity for lithium ion batteries is $40 \%$ which allows for sufficient electrolyte absorption while not compromising the cell's performance. The pore size must be smaller than the particle size of the electrode components making it electrically resistive while still allowing the transfer of ions. The last purpose of a separator is safety; it must be capable of shutting down the battery if something in the cell is disrupted and a thermal runaway leading to combustion. This is why most separators are made up of polyethylene, polypropylene, or a layered mixture of both, enabling a shutdown by thermal runaway at about $130^{\circ} \mathrm{C}$, resulting in a safer cell [6], [40], [41], [42].

The micro porous polymer separator material for lithium ion batteries can be manufactured via a dry or a wet process. Dry processed separators are manufactured by first extruding a polymer resin into a uniaxially oriented layered film. The film is then 
annealed to improve the crystalline structure before formation of the micro-pores, which are formed by uniaxial and biaxial stretching of the film resulting in a distinct slit pore design and straight microstructure (Figure 14-A). The wet manufacturing process for separator materials begins with melting and mixing a polymer resin with paraffin oil and other additives to formulate a homogenous solution. The polymer resin is then extracted, similarly to the dry process, forming a gel type film. Finally, a volatile solvent such as methylene chloride is utilized to remove the paraffin oil and additives in order to form a separator material that exhibits interconnected spherical type pores (Figure 14B) [29], [41], [42].
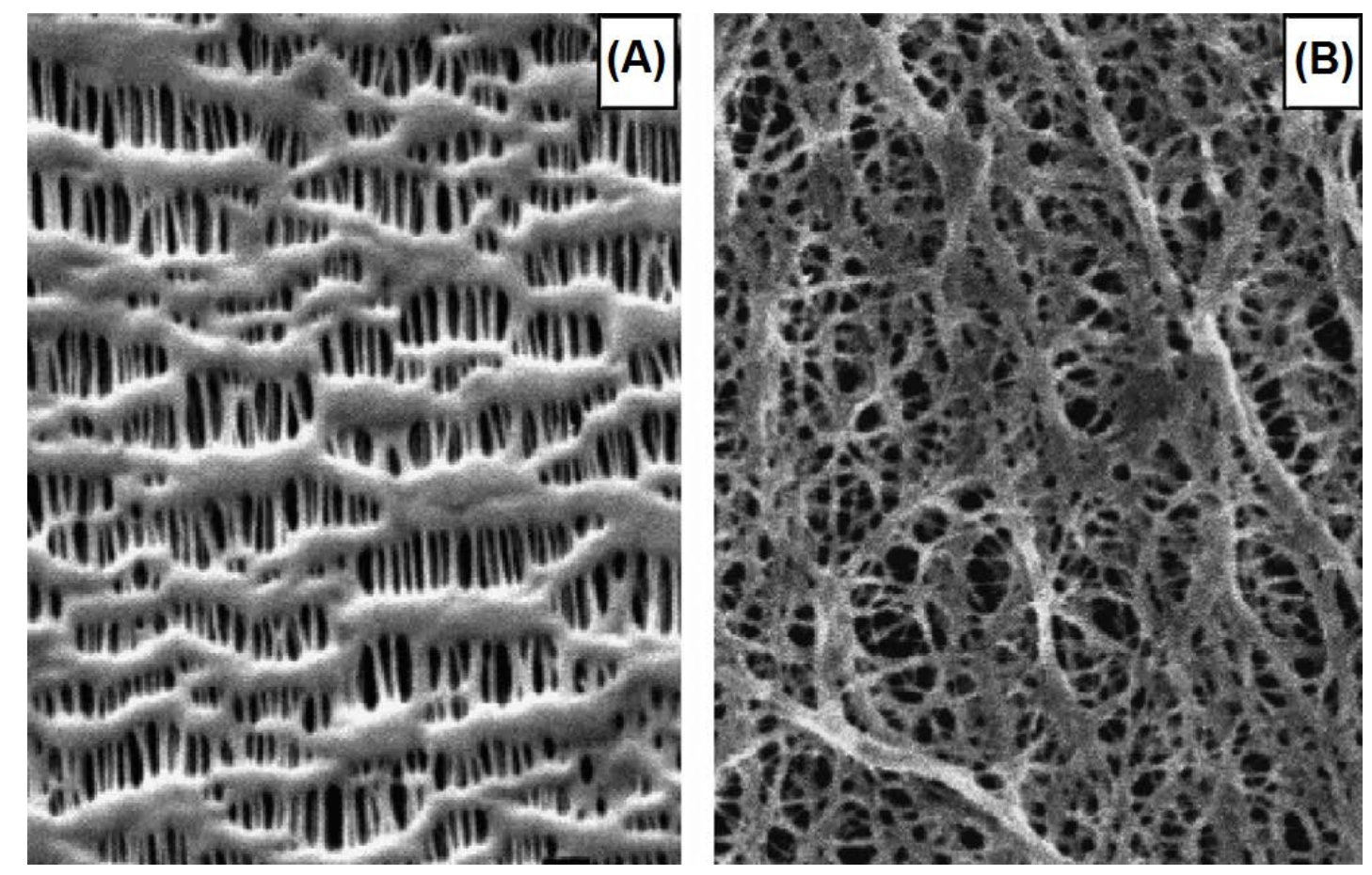

Figure 14 - Microstructure of a micro porous polymer film manufactured by (A) dry process and (B) wet process [41].

\subsection{Battery Testing}

There are several ideas throughout literature of how to properly form and test lithium ion batteries, and how the protocol maximizes the life cycle of a lithium ion battery [43]; however, this section only provides general protocols that are utilized for battery testing with a classical $\mathrm{LiCoO}_{2}$ and graphite cell architecture. The protocol rates are based on the reversible capacitance (C) of $\mathrm{LiCoO}_{2}$ and the voltage range being utilized, which can range from 4.3-2.75 V. However, this range is typically kept between 
4.2-3.0 $\mathrm{V}$ to minimize any capacity loss that can be unintentionally increased by pushing the cathode to its electrochemical limit resulting in a structural degradation and breakdown of active electrode materials [28].

The formation cycles of a newly manufactured lithium ion cell are essential to properly form a highly functional solid electrolyte interface (SEI) layer, with a protocol that typically ranges from a constant current (CC) charge rate of $\mathrm{C} / 20-\mathrm{C} / 40$ for $1-5$ cycles [43], [44]. The SEI layer is best stated as being the most important but least understood part of rechargeable lithium ion batteries, due to fact that the SEl layer is not fixed in its chemistry and has properties that can change drastically with different materials and over time [45]. It is generally believed that the SEI layer is mainly formed during the first cycle of a lithium ion cell where the transfer of lithium ions from the cathode into the anode causes a decomposition of the electrolyte to form the SEI layer and gaseous by-products of $\mathrm{CO}, \mathrm{CO}_{2}, \mathrm{HF}$, and $\mathrm{C}_{2} \mathrm{H}_{5} \mathrm{~F}$ on the anode [46], [47]. This phenomenon causes an initial irreversible capacity loss of $5-30 \%$ depending on the graphite [3], [48]. This reaction causes an electronically insulating layer on the graphite surface but still allows the lithium ions to pass through. If the SEl layer is not sufficiently thick enough, the irreversible capacity loss will increase due to the rapid exfoliation of the graphite structure along with electrolyte decomposition (Figure 15), [44], [49]. Furthermore, the SEI layer is increased with a decrease in particle size, because a smaller particle breaks down faster due to its smaller and weaker structure. This leads to a growth of the SEI layer on more particles resulting in a cell with a higher irreversible capacity loss. The stability of the SEI film determines the safety, power capability, selfdischarge rate, and cycle life of the battery [35], [38], [50]. 


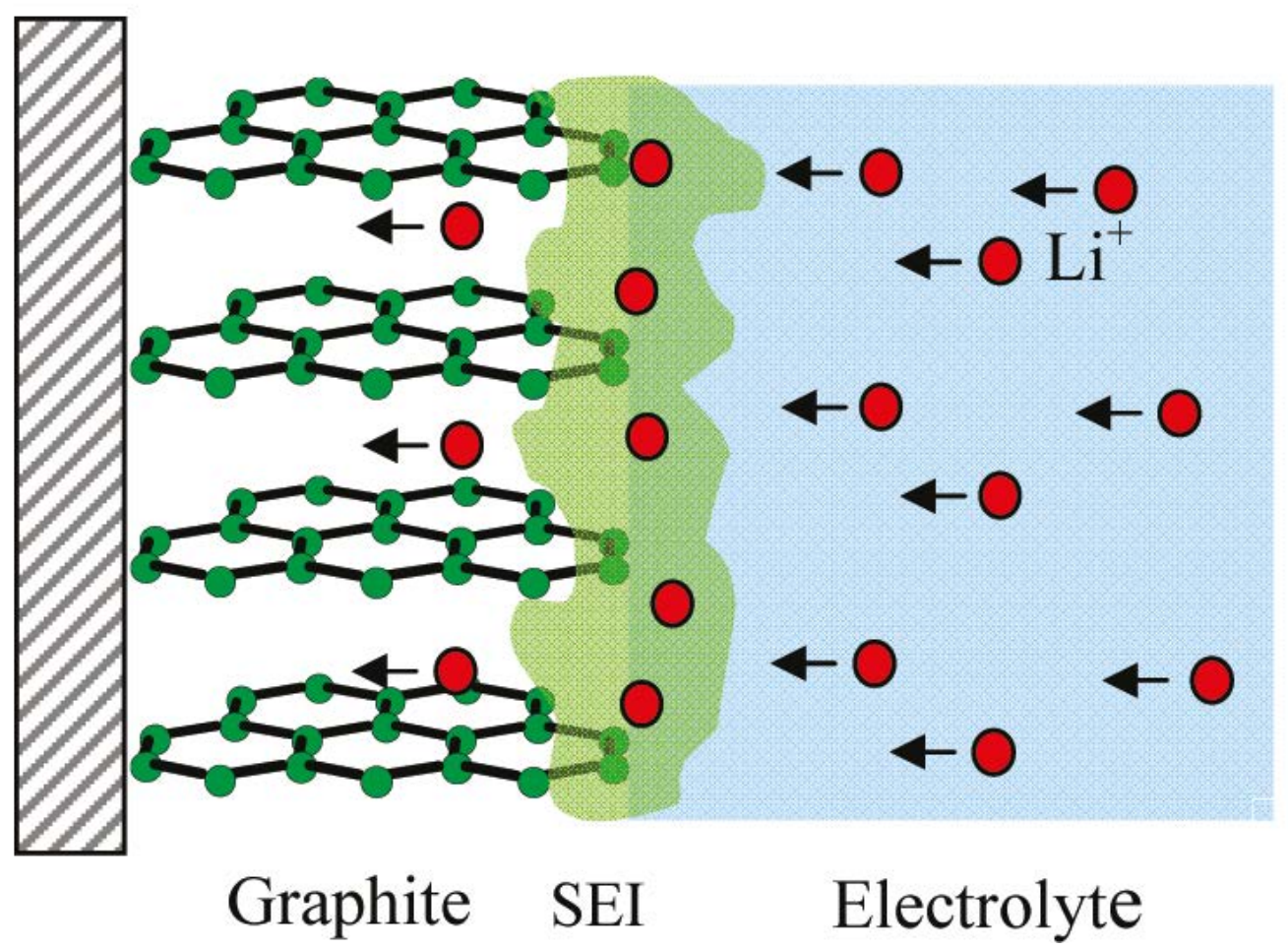

Figure 15 - Schematic of the formation of the SEI layer by electrolyte decomposition of carbonate electrolytes [36].

Once the SEI layer has been formed, the battery can be subjected to cycling. $\mathrm{LiCoO}_{2}$ has a general effective cycling range of $2 \mathrm{C}-\mathrm{C} / 10$, from just 1 cycle to hundreds of cycles, depending on the research and purpose of the cell [43], [51]. The discharge cycle contains only a CC rate from 4.2-3.0 V. The charge cycle also contains a constant voltage $(\mathrm{CV})$ rate, due to the fact that when the lithium ion cell reaches $4.2 \mathrm{~V}$ at a faster $\mathrm{CC}$ rate, it is not fully recharged. This $\mathrm{CV}$ rate is typically held at $4.2 \mathrm{~V}$ until the current from the cell drops to $\mathrm{C} / 20$, or 2 hours have passed to ensure a full recharge of the lithium ions into the cathode. The cutoff of the CV rate is to prevent overcharging and decomposition of the SEI layer, which would subsequently further form the SEI layer lead to a higher irreversible capacity loss [2], [44], [52].

To exhibit the general data collected from testing lithium ion batteries, sample performance graphs of cycled lithium ion batteries are shown in Figures 16-17. These graphs illustrate the data that should be expected of $\mathrm{LiCoO}_{2}$ and graphite electrode cells, as well as the performance of current experimental structural batteries [11], [53]. The normalized capacity fade evident from each cycle is comparable to all similar 
batteries, because it is based on the specific amount of $\mathrm{LiCoO}_{2}$ in the cell. The coulombic efficiency (CE) is the magnitude of the capacity fade and is determined by

$$
\text { Eq. } 1 \text { - } C E=\frac{Q_{D}}{Q_{C}} * 100
$$

( $Q=$ charge, $\mathrm{D}=$ total out, $\mathrm{C}=$ total in) of each cycle [54].

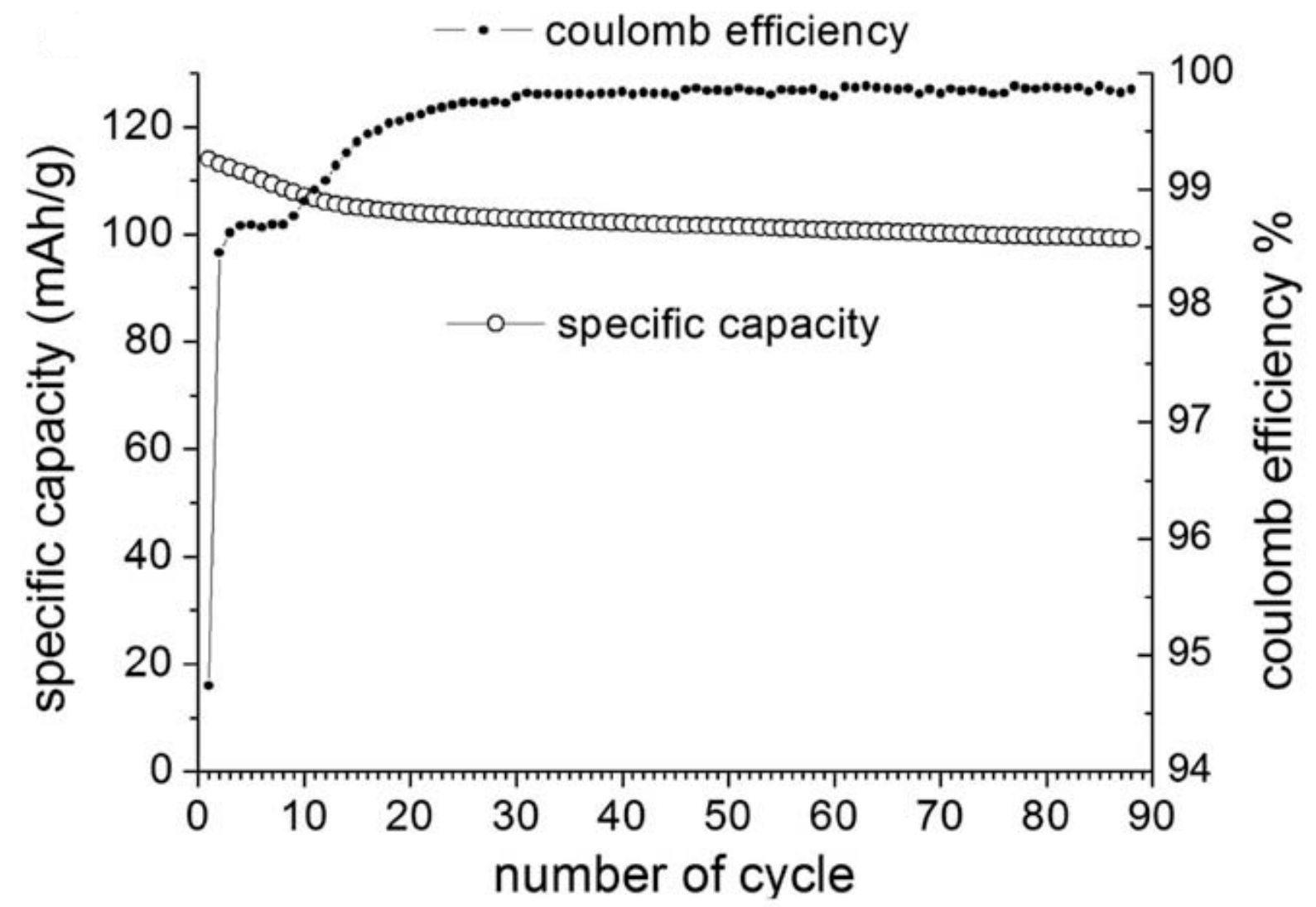

Figure 16 - Specific capacity and coulombic efficiency trend vs. number of cycles of a $\mathrm{LiCoO}_{2} /$ graphite cell cycled at a $1 \mathrm{C}$ rate [53]. 


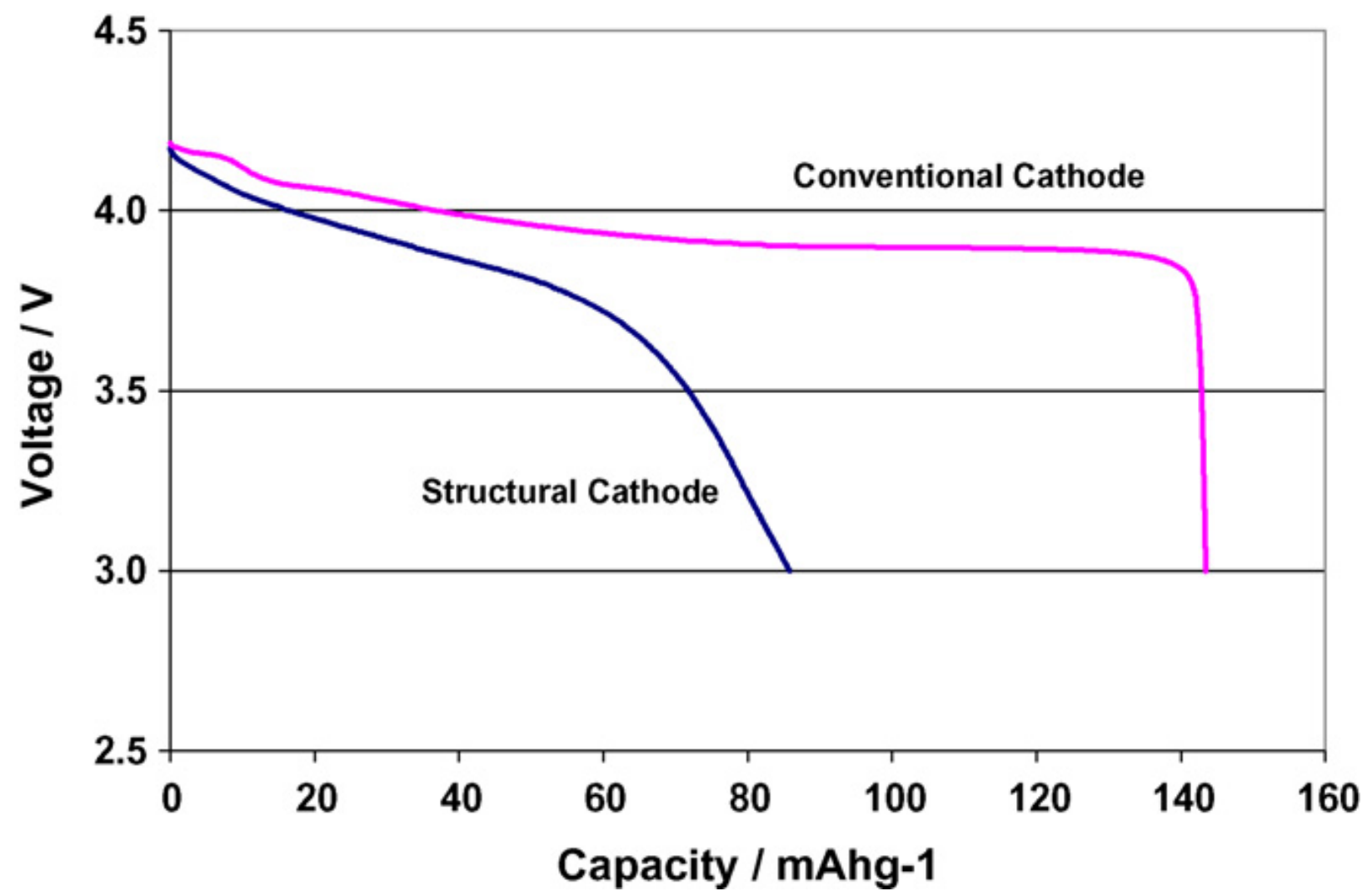

Figure 17 - Conventional lithium ion cathode discharge performance compared to a structural electrode modified with carbon nanofiber [11].

\subsection{Multifunctional Materials}

The largest survey of material properties found through electrochemical testing of fibrous fabrics has been conducted by the US Army Research Laboratory. Their research was comprised of electrochemical testing on readily available commercial carbon materials in novel lithium ion battery structures. Their results showed that PAN based carbon fiber materials resulted with the best overall electrochemical capacitance as well sustaining the widest breadth of applicability for use in structural or textile applications [55]. Other groups have taken this idea further by trying to improve the electrochemical properties of carbon fibers by pretreatments such as heat or chemical modifications. However, most of the tested carbon fiber materials were individually processed, are not commercially available, and each specifically processed material was subjected to a single type of pretreatment [56], [57]. Another way observed throughout literature was to modify the carbon fiber material by pretreating the fibers, then incorporating them within a traditional anode, rather than a continuous weave, to 
increase the electrochemical performance of a traditional lithium-ion cell [58], [59]. The impetus for this research was based on the fact that the electrochemical potential as a standalone anode of many of these different pretreatments on commercially available materials are largely unknown. In this research, these ideas are being manipulated and integrated for the purpose of making a truly composite multifunctional battery.

\subsection{Structural Battery}

Several institutions and companies, such as the US Department of Defense and different aeronautical groups, are currently working in the area of multifunctional structural batteries. Their overall approach uses off the shelf batteries in a structure, instead of truly integrating the battery and structure together. One of their approaches focuses on removing internal polymer components of the functional structure and replacing them with commercial lithium ion cells sandwiched into structural panels. This eliminates the need for a secondary battery subsystem, which in turn reduces the volume and weight of the structure or craft. Testing showed that the alteration of the internal structural components did not compromise the structure's required mechanical properties [10], [60]. Another proven example of combining the structure and battery is in unmanned air vehicles (UAV), as demonstrated in the Wasp micro-air vehicle wing skin. The Wasp design approach added a structure function to already existent systems, by stacking battery cells with a carbon fiber cloth layer between the separate cells, in combination with incorporating a carbon epoxy layer around them (Figure 18). This accomplished a lighter overall structure with enhanced mechanical strength, leading to an overall success that was evident with longer flight times [1], [10], [60]. The approach of this research is to truly integrate the structure and battery into one combined and truly multifunctional component. 


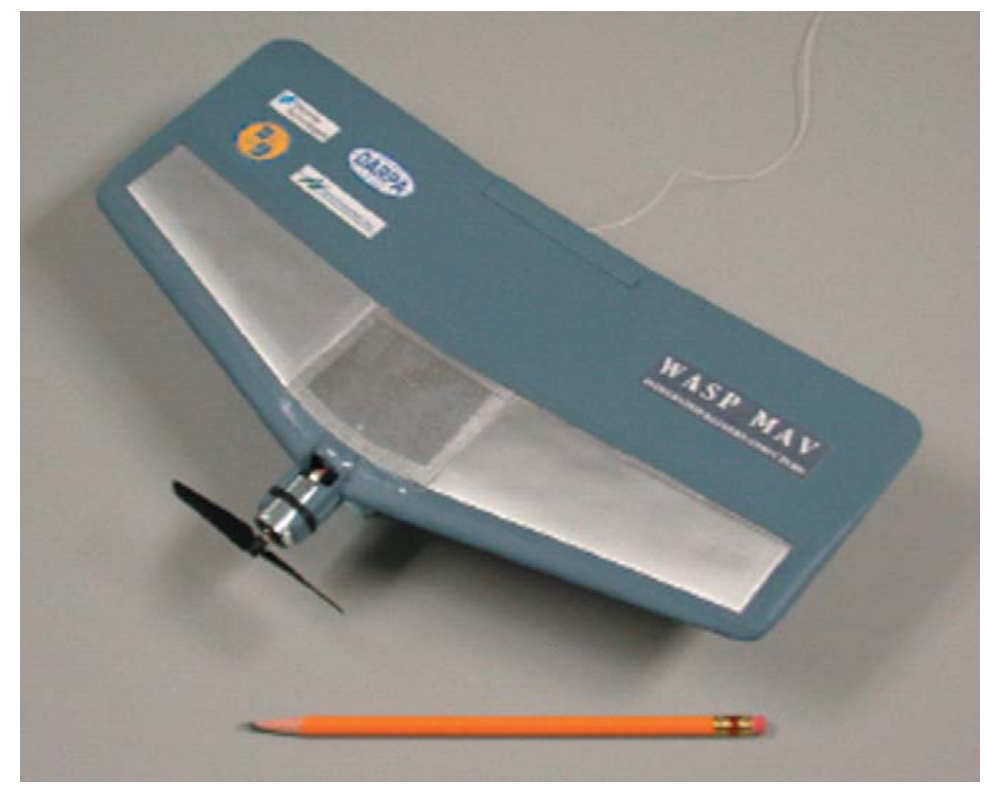

Figure 18 - DARPA Wasp micro-air vehicle with lithium ion pouch cells integrated into the composite wing skin structure [60].

\subsection{Paths of Achievements}

The main objective of this technology is to create a power supply vessel that can maintain its structural integrity while enduring elevated mechanical loads directly to the battery, in addition to optimal electrochemical stability under normal electrical loads and thermal conditions. The combination of both structural and electrochemical functions in a single material permits alternative power system design and applications not possible through each individual component. The design of composite multifunctional structural batteries for optimal performance involves the proper selection of the materials, architecture, and electrical interconnection between them [11]. The approach for achieving the fabrication of a structural multifunctional battery has been broken into distinct parallel paths based on the architecture of the components being implemented within the design.

The first battery architecture (Figure 19) consists of a structure of carbon fibers and fiberglass, typically incorporated within fiber-reinforced composites that act as active components within the battery structure. The structure exemplifies the true intent of a multifunctional battery. This path focuses on electrochemical testing of composite materials, such as carbon fibers and fiberglass in a button cell platform, as well as maximizes the potential for utilization in a structural multifunctional battery. 
The second battery architecture (Figure 20) consists of modifying a typical multifunctional lithium ion pouch battery by creating perforations through the cell for shear reinforcement to reduce delamination. This architecture allows for a highly formable battery, which when layered within a composite matrix will enhance the flexural strength. This path focuses on four-point beam testing to characterize the relationship between the proposed architectural modifications and the battery's structural integrity.

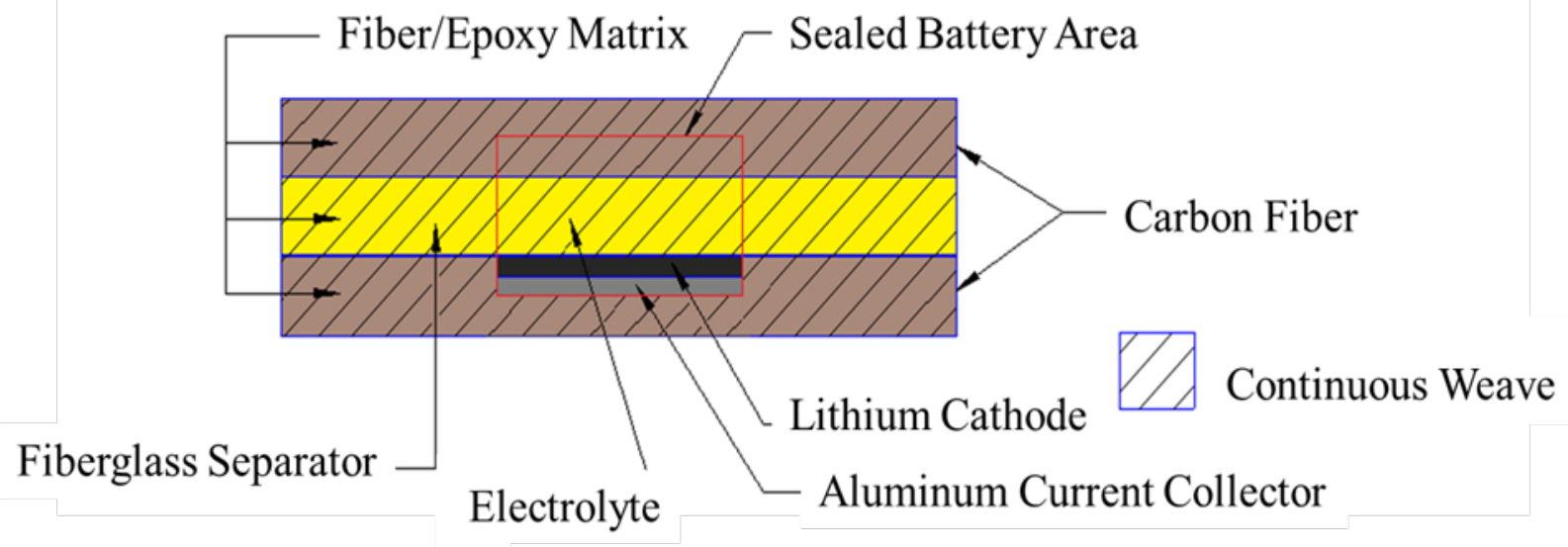

Figure 19 - A schematic of the first battery architecture showing a cell embedded within a laminate composite of continuous fiberglass and carbon fiber weaves running through the structure.

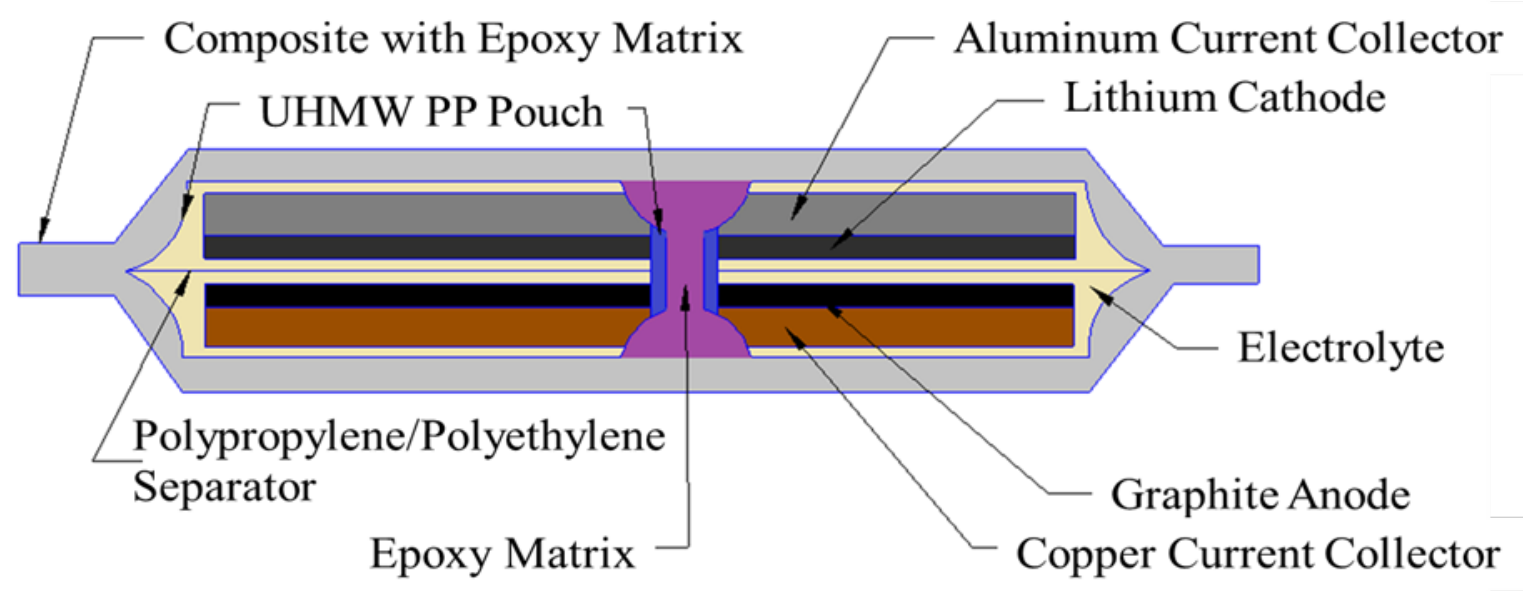

Figure 20 - A schematic cross section of the second battery architecture, which consists of reinforcement vias passing through the active components within a pouch battery; therefore, allowing for further shear reinforcement through the interior of the battery compartment. 


\section{Chapter 3: Fabrication of the Novel Lithium Ion Cells}

\subsection{Introduction}

The overall process of fabricating a single cell battery involves several delicate steps that are combined in order to achieve a functioning cell. The purpose of this chapter is to review and explain all of the procedures and techniques involved in this process.

\subsection{Powder Processing}

There are several different processing methods for the synthesis of in-house active materials for lithium ion batteries, mainly being solid state reactions, hydrothermal synthesis, and sol-gel preparation [6]. The active materials for a classical lithium ion cell may be purchased; however, there are no detailed processing techniques for the companies' respective powders available. The process used in this research is a typical solid state reaction, which is achieved through vigorous mechanical stirring over time [12], [61].

The precursor powders used in the powder synthesis of producing in-house lithium cobalt oxide $\left(\mathrm{LiCOO}_{2}\right)$ were lithium carbonate $\left(\mathrm{Li}_{2} \mathrm{CO}_{3}\right)$ (Fisher Scientific) and Cobalt (II) Carbonate $\left(\mathrm{CoCO}_{3}\right)$ (Alfa Aesa). Calculations for this in-house synthesis were completed based on the precursor powders specific moles, molecular weight (MW), and loss on ignition (LOI) to produce the final desired powder. An illustration of these calculations is shown in Table 1.

Table 1 - Batch calculations for in-house synthesized $\mathrm{LiCoO}_{2}$ cathode powder.

\begin{tabular}{|c|c|c|c|r|r|r|r|r|}
\cline { 2 - 9 } \multicolumn{1}{c|}{} & $\mathrm{LiCoO}_{2}$ & moles & $\mathbf{M W}$ & $\begin{array}{c}\text { moles x } \\
\text { MW }\end{array}$ & $\begin{array}{c}\text { Weight } \\
\text { Fraction }\end{array}$ & Mass [g] & Lol & $\begin{array}{c}\text { Final Mass } \\
\text { [g] }\end{array}$ \\
\hline $\begin{array}{c}\text { Batch Mass } \\
\text { [g] }\end{array}$ & $\mathrm{Li}_{2} \mathrm{CO}_{3}$ & 0.500 & 73.89 & 36.95 & 0.237 & 47.40 & 0.911 & $\mathbf{5 2 . 0 3}$ \\
\hline $\mathbf{2 0 0}$ & $\mathrm{CoCO}_{3}$ & 1.000 & 118.94 & 118.94 & 0.763 & 152.60 & 0.9319 & $\mathbf{1 6 3 . 7 5}$ \\
\hline Total: & \multicolumn{1}{|l}{} & & 155.88 & 1.000 & 200.00 & & $\mathbf{2 1 5 . 7 8}$ \\
\hline
\end{tabular}

Processing of these powders was completed using a solid state synthesis method by attrition milling the powders with an ethanol solvent. The slurry was 
vigorously stirred for four hours to sufficiently achieve particle reaction. After milling, the powder was rinsed with ethanol and dried at $80^{\circ} \mathrm{C}$. Once dry, the powder was processed with a standard 60 mesh sieve for further characterization studies.

\subsection{Calcination Studies}

Calcination of the lithium cobalt oxide powder was the next step required to complete the solid state reaction process. Since the powder was produced completely in-house with a slightly different solid state process than found throughout literature, calcination studies were performed in a range of temperatures between $600^{\circ} \mathrm{C}$ and $800^{\circ} \mathrm{C}$ for 4 hours at each respective temperature in a compact muffle furnace (MTI Corp.). A structural characterization at each of the respective temperatures was performed in an X-Ray diffractometer (Bruker D8 Discover). Each respective scan was analyzed with X'Pert Highscore Plus software and compared to JCPDS reference \#00050-0653 for $\mathrm{LiCoO}_{2}$. This analysis determined that an $800^{\circ} \mathrm{C}$ calcination temperature was required to complete the solid state reaction process and synthesize the desired $\mathrm{LiCoO}_{2}$ rhombohedral structured powder (Figure 21) [12], [62]. 


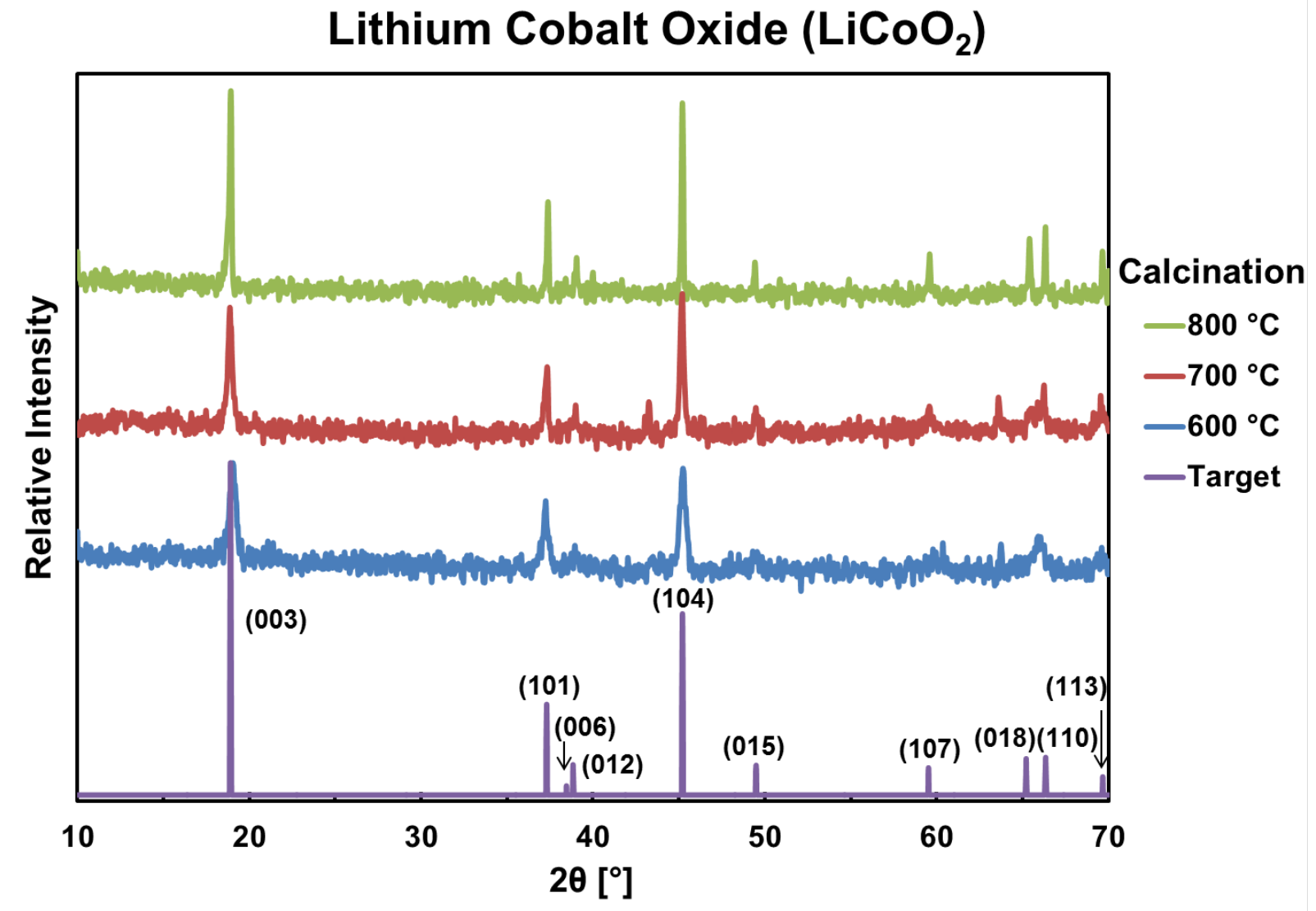

Figure 21 - Overlay of X-Ray diffraction scans of $\mathrm{LiCoO}_{2}$ cathode powder calcinated at different temperatures.

\subsection{Particle Size Analysis}

Throughout literature the particle size for the active cathode powder $\mathrm{LiCoO}_{2}$ ranges from $10 \mathrm{~nm}$ to $8 \mu \mathrm{m}$ and is varied due to the formation process of the powder, as well as the end application in regards to cyclic ability and maximum capacity for a battery. A nanometer sized cathode powder has proved to achieve a higher capacity battery that can be cycled at an elevated rate due to the higher surface area of the particles; however, a much faster fade in the overall capacitance is evident. Furthermore, agglomeration problems occur when synthesizing the electrode inks, which already include nano-sized carbon powders. This makes it difficult to create homogenously dispersed slurries for electrode fabrication [12], [61], [63]. Commercially available $\mathrm{LiCoO}_{2}$ particles are typically produced via solid state means and are available in range of 1-8 $\mu \mathrm{m}$ with an optimal capacitance retention for this research [6], [13], [15]. 
In order to produce an optimum smaller particle size with the synthesized $\mathrm{LiCoO}_{2}$ powder, a second attrition milling process was completed with the calcinated powder. The particle size of the powder was determined in-situ during the milling process with a laser diffraction particle size analyzer (Mastersizer 3000). This machine uses laser diffraction to determine the size of the particles by measuring the intensity of light scattered as a laser beam passes through a dispersed particulate sample, it then takes the refractive index of the powder being observed and calculates the size of the particles as well as the particle size distribution [64], [65]. The milling of the powder continued while measuring the particle size distribution at two hour increments (Figure 22).

\section{$\mathrm{LiCoO}_{2}$ Milled Particle Size Distribution}

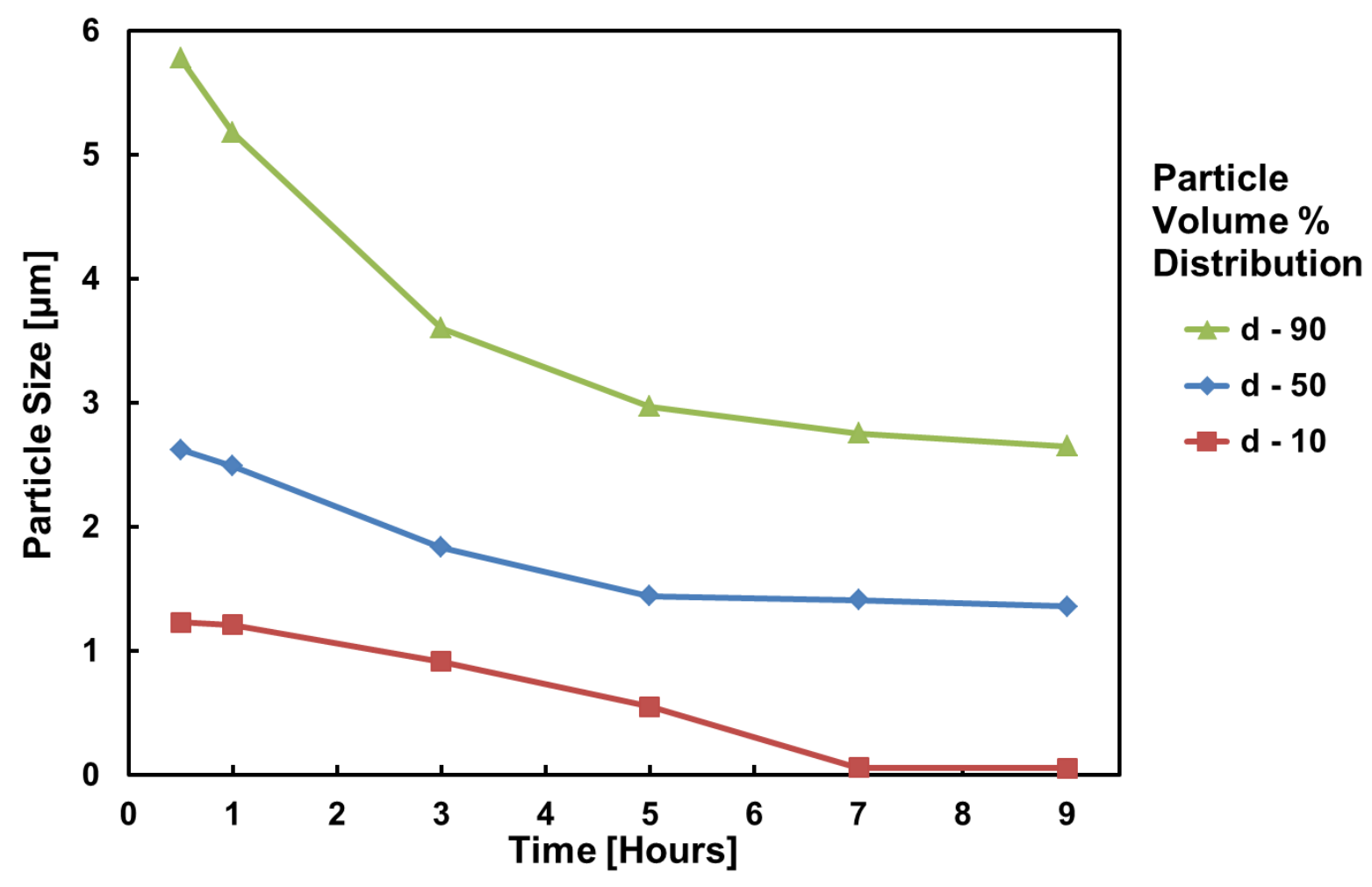

Figure 22 - Average particle size as a function of milling time from 0.5 - 9 hours.

Particle volume percent distribution is the amount of particles in the slurry with a diameter below that respective particle size. The volume distribution of the particle sizes were decreased by the additional attrition milling. This process was conducted for 9 
hours until a smaller average particle size of $1.4 \mu \mathrm{m}$ and a closer particle size distribution was achieved.

\subsection{Active Electrode Particles}

All of the materials utilized in this research, purchased or fabricated, were subject to microstructural characterizations. The two main characterization techniques used for evaluating the materials were scanning electron microscopy (SEM) (JEOL JSM-7600F) to view the architecture of the material, and X-Ray diffraction (Bruker D8 Discovery) to determine the crystalline structure. These techniques were used immensely when comparing purchased battery materials, to novel lithium ion battery components, to inhouse synthesized materials. Focus on characterizing the anode and cathode powders were completed respectively before continuing to the rest of the battery components.

\subsubsection{Graphite}

The graphite powders that were analyzed for use in battery testing consisted of a synthetic type and natural type. The synthetically produced graphite (Sigma Aldrich 282863) contained an average particle size less than $20 \mu \mathrm{m}$, while the natural graphite powder (Alfa Aesar, t2N5) contained an average particle size less than $40 \mu \mathrm{m}$. Throughout literature, both natural and synthetic graphite powders have been proven to be highly functional active anode materials in lithium ion batteries [6], [66], [67], [68]. An analysis to the characterization of these powders (Figure 23 SEM and Figure 24 XRD) is included in this section. 

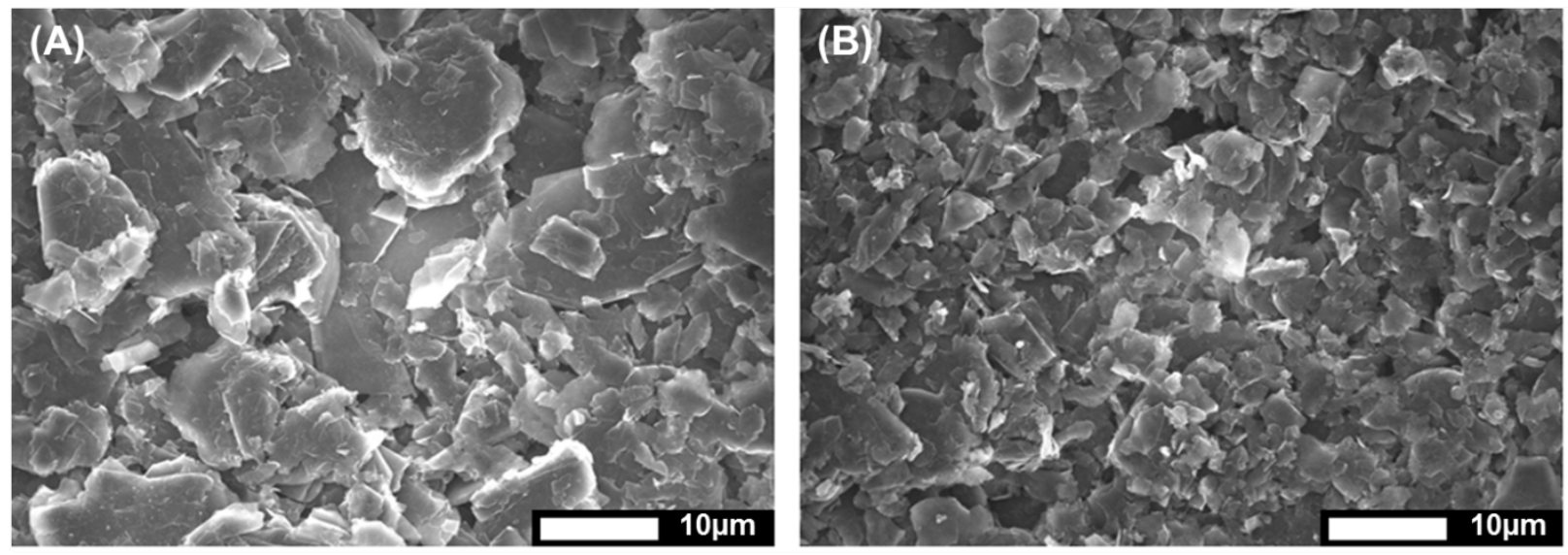

Figure 23 - Scanning electron micrographs of (A) natural graphite powder and (B) synthetic graphite powder.

It is clearly evident from these micrographs that the natural graphite has a much larger particle size, about $15 \mu \mathrm{m}$, than the synthetic graphite powder; however, the flake type morphology of the particles is similar for both powders.

\section{Graphite Powders}

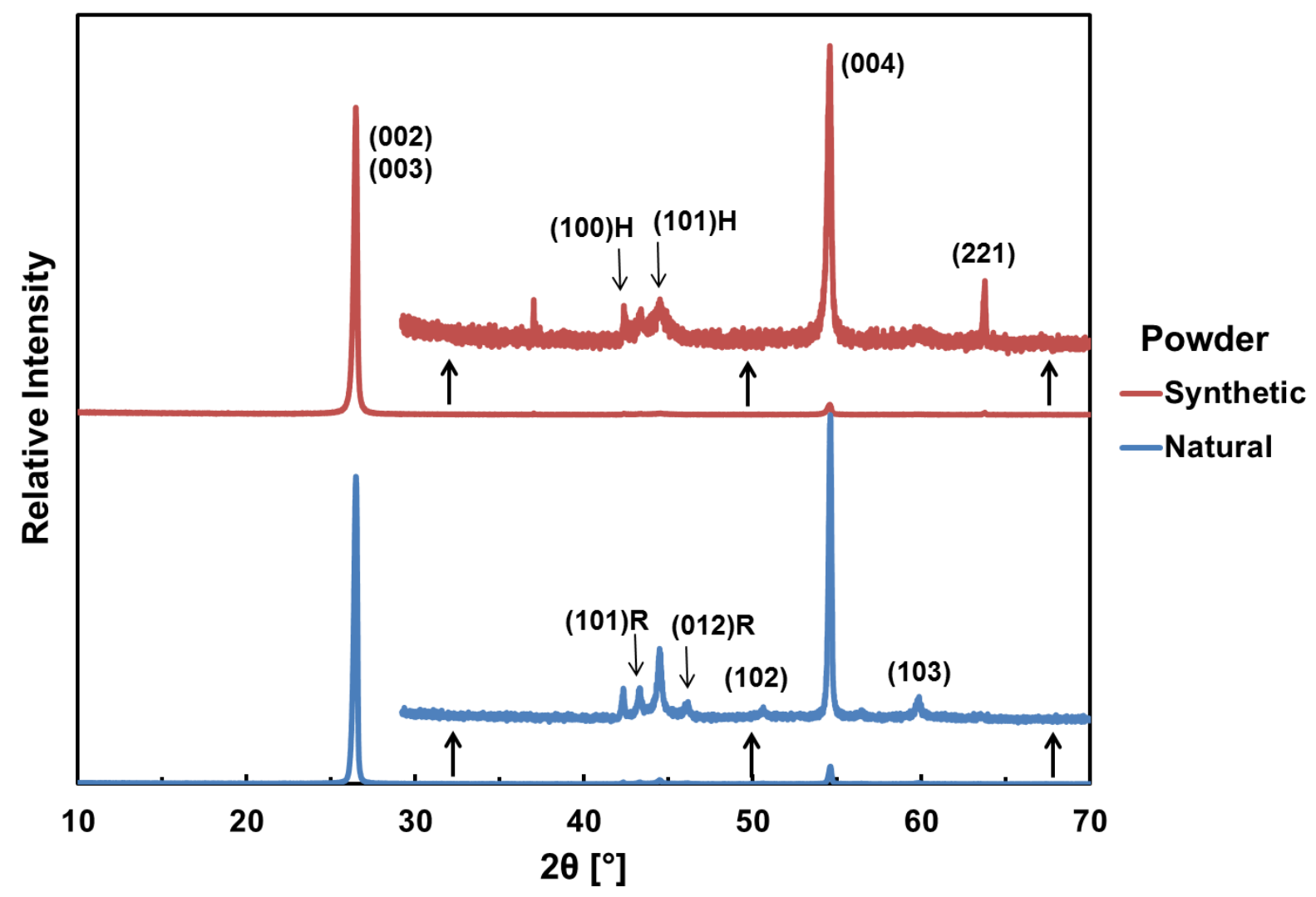

Figure 24 - X-Ray diffraction pattern overlay of natural graphite and synthetic graphite powders. 
The X-Ray diffraction pattern of the two graphite powders demonstrated a distinct difference in their crystal structure. Each graphite contained a high graphitization based on the sharp multi-phase $(002) \mathrm{H}$ (H-hexagonal) and (003) R (R-rhombohedral) crystalline structure peak, both are typical morphologies of graphite. The standard structural phase of graphite is hexagonal with a stacking sequence of $A B A B$. The less common rhombohedral phase is found in both natural and synthetic graphite with a stacking sequence of $A B C A B$ [27], [69]. The volume fraction of the rhombohedral phase can be increased though various mechanical treatments by forming defects in the crystal structure, such as grain boundaries and dislocations. Lithium ions cannot be directly inserted into the hexagonal phase basal plane; therefore, the existence of a rhombohedral phase in graphite promotes more areas for the ions to intercalate into the graphite lattice in the lithium ion battery chemistry [69], [70], [71].

The X-Ray diffraction patterns of the graphite powders were analyzed with X'Pert Highscore Plus software and compared to JCPDS reference \#01-075-2078, as well as JCPDS reference \#03-065-6212. The $2 \theta$ angles between $40^{\circ}$ and $50^{\circ}$ contain reflections seen in both graphite powders of $(100) \mathrm{H}$ at $42.4^{\circ},(101) \mathrm{R}$ at $43.4^{\circ},(101) \mathrm{H}$ at $44.4^{\circ}$, and (012)R at $46^{\circ}$. These peaks were used to determine the volume fraction of each phase present in both graphite powders by quantitative analysis [69], [71].

The quantitative analysis performed utilized reference intensity ratios (RIR) at each specific reflection. These compared the reference pattern intensity to the graphite powder scan intensity. The general form for analysis by the RIR method is:

$$
\text { Eq. 2- RIIR } \boldsymbol{\alpha}_{\alpha, \beta}=\left(\frac{I_{(h k l) \alpha}}{I_{(h k l)^{\prime} \beta}}\right)\left(\frac{r^{r e l}(h k l)^{\prime} \beta}{I^{r e l}(h k l) \alpha}\right)\left(\frac{X_{\beta}}{X_{\alpha}}\right)
$$

Where $I$ is the intensity of the reflection and $I^{r e l}$ is the corresponding reference intensity, while $\alpha$ and $\beta$ refer to the different observed phases. The RIR value is determined by: 


$$
\text { Eq. } 3 \text { - } R I R_{\alpha, \beta}=\left(\frac{R I R_{\alpha, \gamma}}{\operatorname{RIR}_{\beta, \gamma}}\right)
$$

Where $R I R_{\alpha, \gamma}$ and $R I R_{\beta, \gamma}$ are determined by the corresponding reference pattern analysis [72].

This quantitative analysis determined that the synthetic graphite contained a $91.8 \%$ hexagonal phase with an $8.2 \%$ rhombohedral phase. Conversely, the natural graphite contained a $70.1 \%$ hexagonal phase with a $29.9 \%$ rhombohedral phase. In literature it has been suggested that the rhombohedral content, in itself, has no direct influence on the capacity or electrochemical potential, but is strongly dependent on the electrolyte system [70]. Unfortunately, the performance of these two powders in a lithium ion battery chemistry utilized in this research have not been documented in literature, both powders were required to be electrochemically tested (Section 3.11.4) to determine the most relevant one to use as an anode for battery fabrication.

\subsection{2 $\mathrm{LiCoO}_{2}$}

The two cathode materials evaluated in this section are a purchased powder (Sigma Aldrich, 442704) and the in-house synthesized powder. Each of the cathode materials were also subjected to SEM and X-Ray diffraction characterizations. Correlation between each of their respective evaluations is included in this section (Figure 25 SEM and Figure 26 XRD).
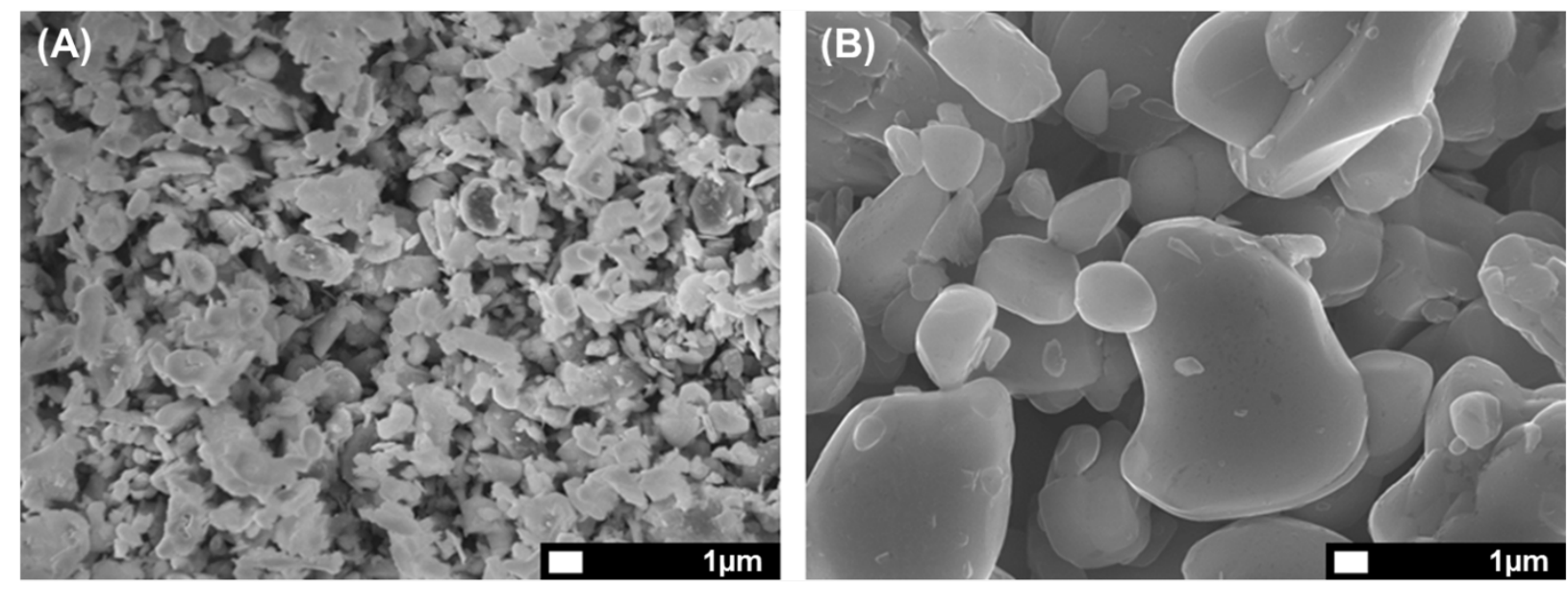

Figure 25 - Scanning electron micrographs of (A) in-house synthesized $\mathrm{LiCoO}_{2}$ and (B) off the shelf $\mathrm{LiCoO}_{2}$ powder. 
There is a visible difference in the morphology of the particles between the inhouse synthesized powder compared to the off-the-shelf powder. This is due to an extra processing step performed to the in-house powder, which consisted of the continuation of the attrition milling step once the powder was calcinated. The purpose of this step was to create a smaller particle size in order to create a higher surface area in the electrode to achieve a higher overall cell capacitance [61].

\section{Lithium Cobalt Oxide $\left(\mathrm{LiCoO}_{2}\right)$ Powder}

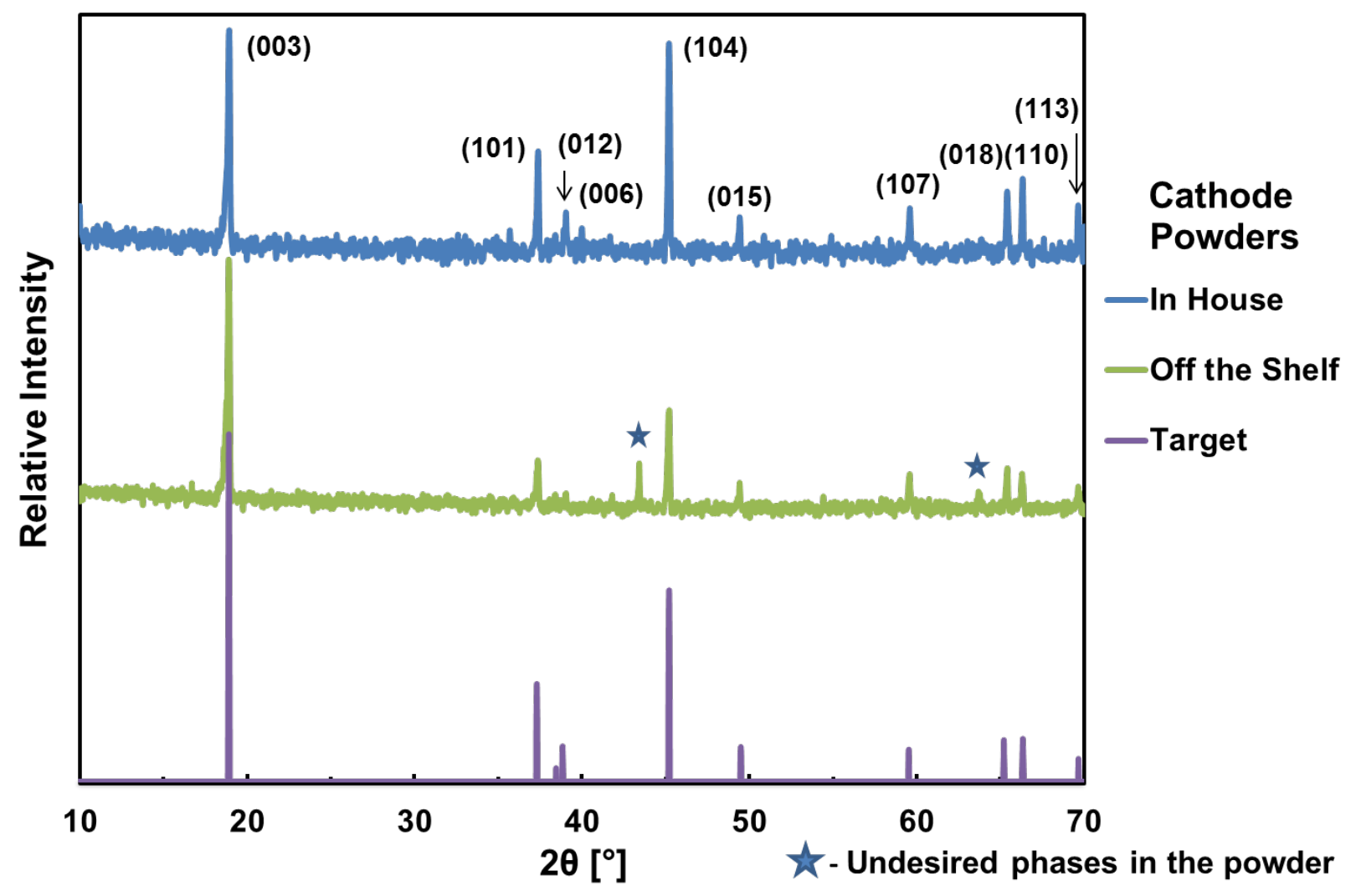

Figure 26 - X-Ray diffraction pattern overlay of the in-house synthesized powder compared to an off the shelf powder.

Extrapolated from this plot is that the lithium cobalt oxide purchased from a supplier is not at the desired $\mathrm{LiCoO}_{2}$ crystal phase for optimum battery performance when compared to JCPDS reference $\# 00-050-0653$. Unfortunately, the initial cell testing performed was on the basis of this powder, which resulted in subpar electrochemical performance data. Fortunately, a pure phase $\mathrm{LiCoO}_{2}$ powder was later obtained from the supplier to use as a reference in comparison to the in-house synthesized cathode. 


\subsection{Formulation of Inks}

In order to utilize the active components of lithium ion batteries, the powders must be incorporated into an ink or liquid slurry state to be deposited onto the interconnect metals. This step combines the active electrode particles with conductive additives and binder in a solvent slurry state that is later deposited onto current collectors as electrodes. There are two main categories for the types of inks used for the fabrication of electrodes in lithium ion batteries, which are water (aqueous) and organic (nonaqueous) based inks. Since all remnants of water must be removed in order for a lithium ion battery to properly function, as well as the powder agglomeration problems due to water based processing, this research focused on the use of a nonaqueous system based on an N-Methyl-2-pyrrolidone (NMP) solvent. There are several formulation variations for a typical nonaqueous ink system, but all of them include an active material, conductive additive, solvent, and binder. The variations of these formulas are due to the particular properties of each individual component's particle size, surface area, and conductivity. Furthermore, the solids loading from all of these particles combined with the method used for synthesizing the ink affects the viscosity and rheology of resulting depositions onto current collectors [6], [73]. Since there is no exact documented formulation of all the particles used in this research, there were several attempts conducted to form an effective ink. The ultimate goal was to synthesize a uniformly dispersed ink that was fluent and able to be successfully deposited onto a current collector.

\subsubsection{Sonication}

The initial inks contained the off-the-shelf active materials, which include the following: lithium cobalt oxide (Sigma Aldrich, 442704) and synthetic graphite powder (Sigma Aldrich, 282863) (which was previously analyzed and discussed in Section 3.5), conductive additive of acetylene carbon black (Strem Chemicals, 06-0025) with a particle size of $42 \mathrm{~nm}$, a binder of polyvinylidene fluoride (PVDF) (Alfa Aesar, 44080) comprised of a 500,000 molecular weight, and a solvent of NMP.

Sonochemical synthesis was conducted with an ultrasonic probe (Sonics VCX130PB) at a low intensity of $20 \%$. Higher intensities of ultrasound irradiation to the 
ink slurry would result in cavitation (implosive collapse of bubbles), which leads to localized heating, undesirable reactions, and an even higher agglomeration of the nanoparticles [74]. The ink formulation used for this initial process was derived from literature [3], in which the cathode consisted of $\mathrm{LiCoO}_{2}+$ acetylene carbon black + PVDF at (88/4/8 wt\%), while the anode consisted of synthetic graphite + PVDF at (92/8 wt\%).

The sonochemical synthesis process proved successful and resulted in functioning electrodes; however, the resulting slurries had to be small and the particles were poorly dispersed. Furthermore, the only deposition process applicable with the resulting ink that functioned was brush painting. This process did prove that a functional battery could be fabricated and tested in-house, but consistency from one ink to another was problematic, so a more reliable method was implemented.

\subsubsection{Solid State Synthesis}

Synthesis of consistent ink batches is essential for producing batteries for empirical comparison from one batch to the next. A solid state method of ball milling was selected for ink synthesis due to its effectiveness and subsequent homogeneous mixture of particles. Once again, the exact chemicals being used in this research are slightly different than the ones found in ink formulations throughout literature. A tactical trial and error process was performed to determine the best ratio for all of the materials in the inks [6].

These inks contained the fabricated lithium cobalt oxide or graphite powder (Alfa Aesar, t2N5) previously analyzed (Section 3.5), two conductive additives: carbon nanofiber (Pyrograf Products, PR-19-XT-LHT) with a particle length of $150 \mathrm{~nm}$, and carbon black (courtesy of Timcal, Super C45) with a particle size of $45 \mathrm{~nm}$, a PVDF binder (Alfa Aesar, 44080) which has a molecular weight of 500,000, and a solvent of NMP (J.T. Baker, 9261-09). This carbon black replaced the previously used acetylene carbon black, as a conductive additive, due to its proven abilities throughout literature (Figure 27-A) [6], [75]. Carbon nanofiber was utilized to elevate electrical conductivity along with the carbon black (Figure 27-B). These nanofibers, in addition to the PVDF binder, incorporated a nanoparticle structural reinforcement component to the 
electrodes that helped bind the particles together, as well as increase the packing factor to form a dense multifunctional electrode system [11].
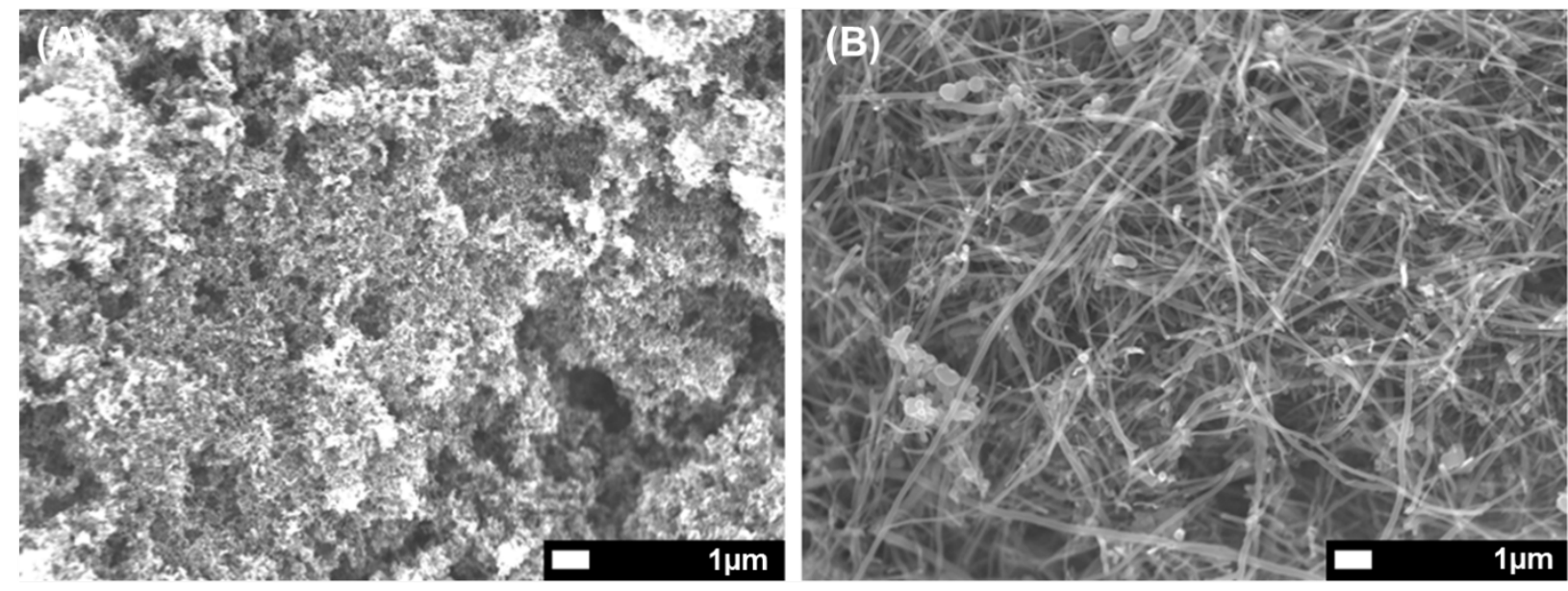

Figure 27 - Scanning electron micrographs of conductive additives (A) carbon black (Timcal, Super C45) and (B) carbon nanofiber (Pyrograf Products, PR-19-XT-LHT).

To better visualize the amount of each powder in the ink slurries, each component was calculated by its volume percent in the slurry instead of its weight percent. Since the deposition method selected was tape casting, which under optimal circumstances requires a high 30-40 vol\% solids loading, the initial ink batches contained a solids loading of 30 vol\% solid material to liquid solvent [17], [76]. However, due to the nature of the nanosized particles utilized, the resulting depositions of the electrodes suffered from severe agglomeration of the particles and poorly dispersed electrode sheets [77]. In order to produce a fluent homogenous ink, the required solids loading of the synthesized slurries was between 10-20 vol\% [3], [17], [78].

The ball milling solid state synthesis was conducted over a 12 hour span at 200 RPM, where all of the materials were homogenously mixed together with the solvent for 11 hours with the binder material added during the last hour. This step was required for the binder to perform its true purpose of connecting all of the particles together. When the binder was milled for the entire synthesis, the longer polymer chained molecules of the binder broke down. This reduced its effectiveness, which lead to ink depositions that were poorly adhered to the current collector foils and resulted in unusable electrodes. 
The resulting ink batch calculations used for the fabrication of lithium ion test cells are shown below (Table 2).

Table 2 - Batch calculations for solid state synthesized electrode inks.

\begin{tabular}{|c|c|c|c|c|c|c|}
\hline Cathode & $\begin{array}{l}\text { Ratio } \\
\text { [g] }\end{array}$ & $\begin{array}{l}\text { Weight } \\
{[\%]}\end{array}$ & $\begin{array}{l}\text { Density } \\
{\left[\mathrm{g} / \mathrm{cm}^{3}\right]}\end{array}$ & $\begin{array}{l}\text { Volume } \\
{\left[\mathrm{cm}^{3}\right]}\end{array}$ & $\begin{array}{l}\text { Volume } \\
\text { [\%] }\end{array}$ & Batch Mass [g] \\
\hline $\mathrm{LiCoO}_{2}$ & 0.861 & 86.091 & 2.8 & 30.75 & 80 & 8.61 \\
\hline Carbon Black & 0.031 & 3.075 & 1.6 & 1.92 & 5 & 0.31 \\
\hline Carbon Nanofiber & 0.040 & 4.035 & 2.1 & 1.92 & 5 & 0.40 \\
\hline PVDF & 0.068 & 6.799 & 1.77 & 3.84 & 10 & 0.68 \\
\hline Solvent $_{\text {Calculated: }}$ NMP & 1.25 & 125 & 1.028 & 121.60 & 316.40 & 12.50 \\
\hline Solvent $_{\text {Real: }}$ NMP & 2.2 & 220 & 1.028 & 214.01 & 556.86 & 22.00 \\
\hline Total & 1 & 100 & & 38.43 & 100.00 & 10.00 \\
\hline Solids Loading & & & & & 17.96 & \\
\hline
\end{tabular}

\begin{tabular}{|c|c|c|c|c|c|c|}
\hline Anode & $\begin{array}{l}\text { Ratio } \\
\text { [g] }\end{array}$ & $\begin{array}{l}\text { Weight } \\
\text { [\%] }\end{array}$ & $\begin{array}{l}\text { Density } \\
{\left[\mathrm{g} / \mathrm{cm}^{3}\right]}\end{array}$ & $\begin{array}{l}\text { Volume } \\
{\left[\mathrm{cm}^{3}\right]}\end{array}$ & $\begin{array}{l}\text { Volume } \\
\text { [\%] }\end{array}$ & Batch Mass [g] \\
\hline Graphite & 0.850 & 85.025 & 2.25 & 37.79 & 82 & 8.50 \\
\hline Carbon Black & 0.029 & 2.946 & 1.6 & 1.84 & 4 & 0.29 \\
\hline Carbon Nanofiber & 0.039 & 3.869 & 2.1 & 1.84 & 4 & 0.39 \\
\hline PVDF & 0.082 & 8.16 & 1.77 & 4.61 & 10 & 0.82 \\
\hline Solvent $_{\text {Calculated: NMP }}$ & 1.5 & 150 & 1.028 & 145.91 & 316.64 & 15.00 \\
\hline Solvent $_{\text {Real: }}$ NMP & 3.846 & 384.6 & 1.028 & 374.12 & 811.85 & 38.46 \\
\hline Totals & 1 & 100 & & 46.08 & 100.00 & 10.00 \\
\hline Solids Loading & & & & & 9.60 & \\
\hline
\end{tabular}

The resulting ink formulation determined by the trial and error process was a cathode that contained $\mathrm{LiCoO}_{2}$ (in-house-fabricated) + carbon black (Timcal, Super C45) + carbon nanofiber (Pylograph, PR-19-XT-LHT) + PVDF (Alfa Aesar, 44080) at (80/5/5/10 vol\%), and an anode that consisted of natural graphite (Alfa Aesar, t2N5) + carbon black (Timcal, Super C45) + carbon nanofiber (Pylograph, PR-19-XT-LHT) + PVDF (Alfa Aesar, 44080) at (82/4/4/10 vol\%). The battery performance tests that determined the cathode and anode ink ratio of materials are discussed in (Section 3.11.4), whereas this formulation was utilized to fabricate electrodes for the rest of the battery tests. 


\subsubsection{Rheology}

The rheology between batches of the cathode and anode inks were an important procedure performed to ensure consistency between the solid-liquid dispersion from one ink batch to the next. This was executed to eliminate any unknown mishaps throughout the processing conditions of the ink, which would result in an electrode cast that would not be comparable to the others in battery testing [15], [73]. Additionally, the viscosity of the inks must be low in order to ensure a homogeneous flow during the casting process [17].

The rheological properties of the inks were tested in a controlled rate viscometer (Brookfield DV-II+ PRO). An increasing sheer rate was executed while measuring the sheer stress and viscosity in centipoise. The average viscosity of the cathode was around $1200 \mathrm{cps}$ while the anode was about $1600 \mathrm{cps}$ at a sheer rate of $20 \mathrm{~s}^{-1}$, which was the lowest speed possible that provided consistent results. All of the inks fabricated for battery performance tests were within $\pm 100 \mathrm{cps}$ of the average viscosity.

\subsection{Electrode Deposition}

There are several proven techniques for fabricating the electrodes for lithium ion batteries. Throughout this research, painting, screen printing, spin coating, and tape casting were methods experimented with to deposit the electrodes [6].

The first method for fabricating the electrodes was painting the ink onto a precut foil with a small paint brush. All of the electrodes were then dried in an oven at $80^{\circ} \mathrm{C}$ and weighed after drying to determine if another coat was needed to have all of the electrodes as close to the same mass as possible. This electrode method was tested in a three electrode split battery cell for proof that a functioning cathode and anode were both produced; furthermore, the carbon fiber and fiberglass were also tested with this process for proof of concept of this research project. The results are shown and discussed in Section 3.11.1. This method proved that a functional cell could be produced, but there were many disadvantages with this process, such as a ridged surface on the electrodes due to the brush strokes, difficulty reproducing similar 
weighted electrodes, and the extended time for processing (due to the fact that each electrode had to be done one at a time).

The processes of screen printing and spin coating were also investigated due to the fact that a much more consistent electrode could be replicated with these methods; however, each of them had their own obstacles as well. The biggest downside for both of these processes is that each of the electrodes would still have to be manufactured individually. Additionally, for screen printing, the graphite particle size used was too big for the in-house mesh size of the screens being used and would not pass fluently through to the electrode. Also, for spin coating, the viscosity of the fluid ink was much too high making it difficult to produce an even coated electrode surface. Both of these methods were experimented, but were not used due to their large shortcomings.

The most effective method investigated to manufacture the electrodes used in this research was by tape casting with a 3 inch doctor blade. The best attributes and reason of utilization of this technique was the ability to fabricate several uniform electrodes at once, along with replicating the results to produce the amount of electrodes needed for all of the battery tests. The only noticeable disadvantage from this technique was a larger amount of material was wasted compared to the other methods.

Since the in-house fabricated inks used for this research are different than that found throughout literature, a trial and error process was executed to determine the cast thickness and drying protocol of the inks. The initial casts were used to determine the electrolyte formulation (Sections 3.8 and 3.11.2), and were cast at $0.3 \mathrm{~mm}$, then dried to $0.01 \mathrm{~mm}$. These casts were used to produce successful cells, but yielded a much lower capacity when compared to the capacity of lithium cobalt and graphite systems found in literature [28], [34]. To determine the optimum tape cast conditions, several casts were conducted varying the casting thickness, conductive additives, and graphite used in the anode to derive the protocol for casting and drying. The cell performance data and microstructure characterization of this optimization of casts are shown and discussed in cast thickness/anode material performance testing (Section 3.11.4). These results determined that a tape casting thickness of $1 \mathrm{~mm}$ for both electrodes, as well as utilizing 
natural graphite (Alfa Aesar, 44080) with carbon additives for the anode yielded the highest capacitance.

The current collectors used were $50.8 \mu \mathrm{m}$ thick; aluminum was used for the cathode and copper for the anode. To ensure a clean surface was being used, the foils first went through a washing step within an ultrasonic bath of $1 \mathrm{M}$ nitric acid for 5 minutes to clear off any foreign particles through a light chemical etching of the surface. This was followed by an ultrasonic acetone bath for 5 minutes to finish cleaning the surface. Next the foils were dipped in the solvent NMP before casting to prevent any corrosion from the air to the clean bare metal surface. Finally, the foils were tightly taped onto a flat piece of tempered glass to create a flat surface for casting and to keep the foils from curling during the drying process (Figure 28).
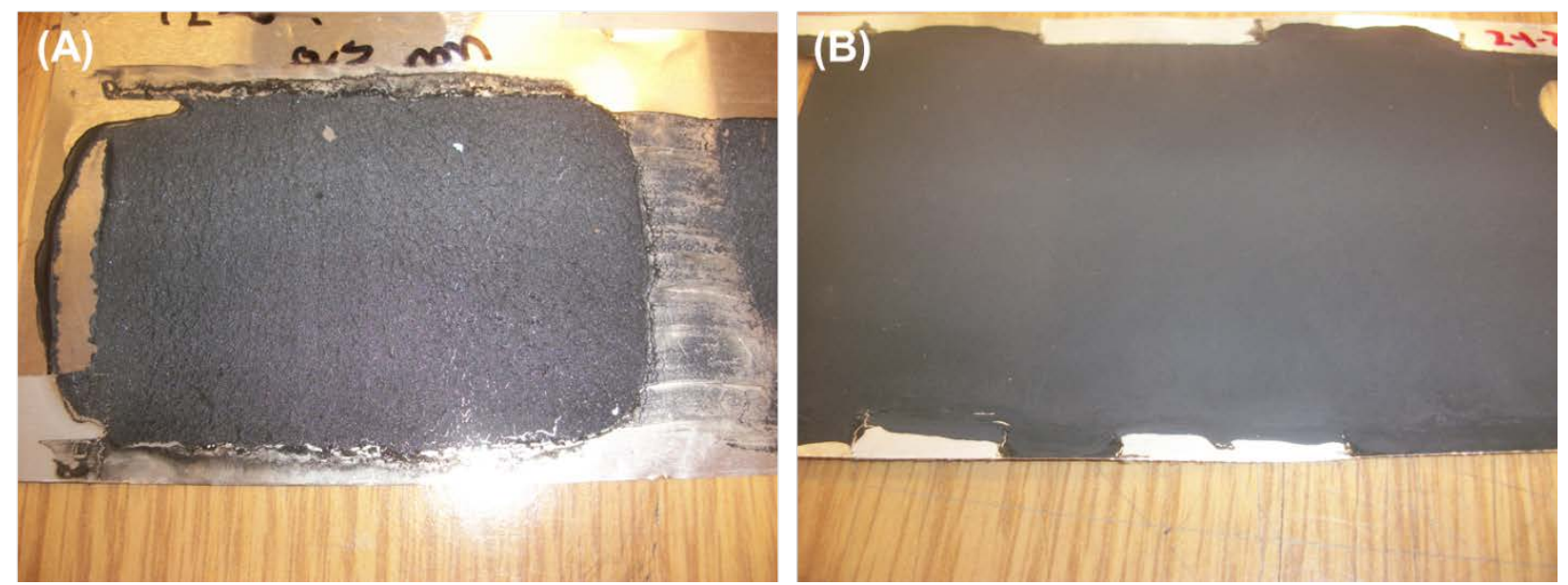

Figure 28 - Tape casted cathode electrodes for lithium ion batteries. (A) Unsuccessfully fabricated electrode and (B) successfully manufactured electrode.

The drying process for these tape casts had to be extended from what was found in literature due to the thickness of the cast being deposited, along with the low solids loading derived from the ink being used [21], [76]. If the cast was dried too quickly an agglomeration effect was observed by visual cracking throughout the cast along with poor adhesion to the current collectors (Figure 28-A). Slower drying of the tape casts at a lower temperature under vacuum allowed the particles in the inks to better condense as the solvent was being removed, thus creating a better bond to the foil surface, as well as throughout the entire cast creating a stable composite electrode (Figure 28-B). 
The resulting protocol determined though testing consisted of slow tape casting (by hand) to a thickness of $1 \mathrm{~mm}$. The tapes were then dried under $15 \mathrm{inHg}$ vacuum at room temperature $22^{\circ} \mathrm{C}$ for 12 hours to slowly remove the solvent. Heat was then applied at $100^{\circ} \mathrm{C}$ for 12 more hours under vacuum to finish removing the solvent and any moisture in the casts. The resulting casts produced a $0.24 \mathrm{~mm}$ thick anode and a $0.16 \mathrm{~mm}$ thick cathode. This protocol was utilized for the rest of the battery performance tests.

\subsection{Electrolyte Formulation}

Once a functional cell was manufactured, multiple electrolytes were investigated. There are several proven combinations of electrolyte systems found throughout literature, and in this research four of them were investigated [6], [11]. All of the experiment electrolyte formulations contained a $1 \mathrm{M}$ lithium salt of lithium hexafluorophosphate $\left(\mathrm{LiPF}_{6}\right)$ (Strem Chemicals, 03-0325) in different electrolyte carbonate solutions of ethylene carbonate (EC) (Alfa Aesar, A15735), diethyl carbonate (DEC) (Alfa Aesar, A12477), dimethyl carbonate (DMC) (Alfa Aesar, A13104), and propylene carbonate (PC) (Alfa Aesar, A15552).

The first electrolyte system investigated for the initial testing was $1 \mathrm{M} \mathrm{LiPF}_{6}$ in $E C+D E C+D M C+P C(4: 3: 2: 1$ vol\%) solution [11]. EC is a transparent crystalline solid at room temperature, so it had to be slightly heated to its liquid state for adding into the solution; however, once it was mixed with the other carbonate solutions it remained in liquid form. Furthermore, the lithium salt is extremely reactive with moisture so it was not added to the carbonate solution until it was transferred into the glovebox, where it was mixed into the solution with a stir rod and stir plate for approximately an hour. This initial system only functioned at low rates and was extremely unstable in a classical cell format. This was due to the reaction between propylene carbonate and crystalline graphite during the charging process, thus causing exfoliation of the graphite anode and resulted in a plateau around $3.2 \mathrm{~V}$ during the initial charge. This phenomenon destroyed the anode's infrastructure that attributed to electrical isolation of the cracked graphite particles and deactivation of the anode [37], [39]. 
Once it was determined that PC caused the problem within the cells, three other electrolyte systems found commonly throughout literature were synthesized, and these were as follows: $1 \mathrm{M} \mathrm{LiPF}_{6}$ in EC+DEC (3:7 vol\%), 1M LiPF 6 in EC+DEC (1:1 vol\%), and $1 \mathrm{M} \mathrm{LiPF}_{6}$ in EC+DEC+DMC (1:1:1 vol\%). These systems were simultaneously tested and compared with one another at a $1 \mathrm{C}$ rate. The results of this testing are shown and discussed in electrolyte performance testing (Section 3.11.2). Formulation for the different electrolyte batches are shown in Table 3 [6], [56].

Table 3 - Synthesized electrolyte batch calculations.

$1 M \mathrm{LiPF}_{6}$ in $\mathrm{EC}+\mathrm{DEC}+\mathrm{DMC}+\mathrm{PC}(4: 3: 2: 1 \mathrm{vol} \%)$

\begin{tabular}{|l|c|c|c|r|r|r|}
\hline \multicolumn{1}{|c|}{ Chemicals } & Formula & $\begin{array}{c}\text { Molecular } \\
\text { Weight } \\
\text { [g/mol] }\end{array}$ & $\begin{array}{c}\text { Density } \\
{[\mathbf{g} / \mathbf{m L}]}\end{array}$ & $\begin{array}{c}\text { Volume } \\
\text { Ratio }\end{array}$ & $\begin{array}{c}\text { Volume } \\
{[\mathbf{m L}]}\end{array}$ & $\begin{array}{c}\text { Amount of } \\
\text { Chemical } \\
\text { [g] }\end{array}$ \\
\hline Ethylene Carbonate & $\mathrm{C}_{3} \mathrm{H}_{4} \mathrm{O}_{3}$ & 88.06 & 1.32 & 4.00 & 4.00 & $\mathbf{5 . 2 8}$ \\
\hline Diethyl Carbonate & $\mathrm{C}_{5} \mathrm{H}_{10} \mathrm{O}_{3}$ & 118.13 & 0.98 & 3.00 & 3.00 & $\mathbf{2 . 9 3}$ \\
\hline Dimethyl Carbonate & $\mathrm{C}_{3} \mathrm{H}_{6} \mathrm{O}_{3}$ & 90.08 & 1.07 & 2.00 & 2.00 & $\mathbf{2 . 1 4}$ \\
\hline Propylene Carbonate & $\mathrm{C}_{4} \mathrm{H}_{6} \mathrm{O}_{3}$ & 102.09 & 1.20 & 1.00 & 1.00 & $\mathbf{1 . 2 0}$ \\
\hline $\begin{array}{l}\text { Lithium } \\
\text { Hexafluorophosphate }\end{array}$ & $\mathrm{LiPF}_{6}$ & 151.91 & 1.55 & & & $\mathbf{1 . 5 2}$ \\
\hline
\end{tabular}

Total Batch Mixture [mL]

10

1M LiPF 6 in EC+DEC (3:7 vol\%)

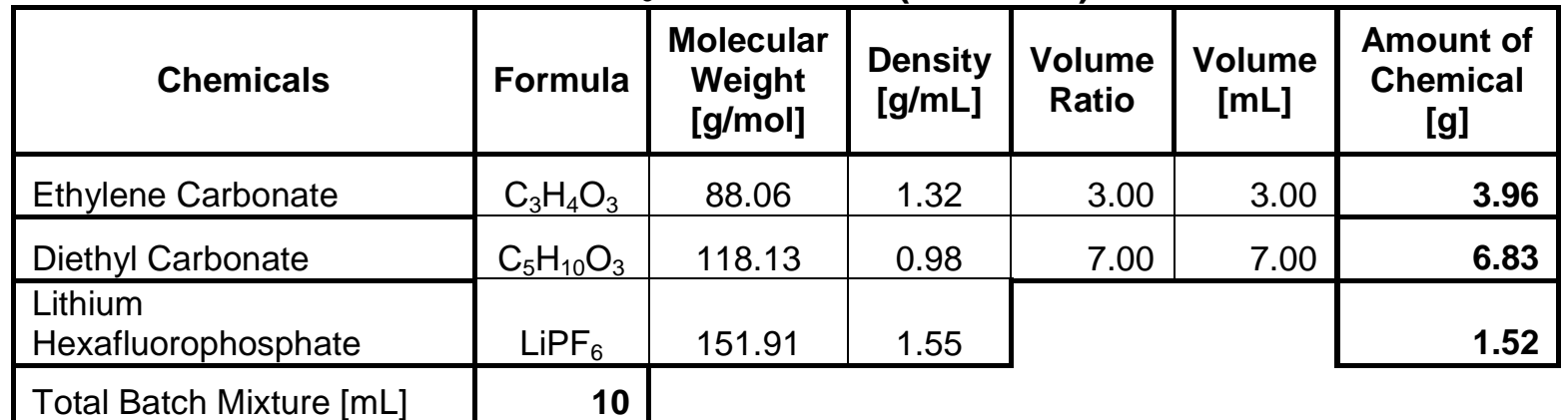

Total Batch Mixture [mL]

10 
$1 \mathrm{M} \mathrm{LiPF}$ in EC+DEC+DMC (1:1:1 vol\%)

\begin{tabular}{|c|c|c|c|c|c|c|}
\hline Chemicals & Formula & $\begin{array}{c}\text { Molecular } \\
\text { Weight } \\
\text { [g/mol] }\end{array}$ & $\begin{array}{l}\text { Density } \\
{[\mathrm{g} / \mathrm{mL}]}\end{array}$ & $\begin{array}{l}\text { Volume } \\
\text { Ratio }\end{array}$ & $\begin{array}{l}\text { Volume } \\
{[\mathrm{mL}]}\end{array}$ & $\begin{array}{l}\text { Amount of } \\
\text { Chemical } \\
\text { [g] }\end{array}$ \\
\hline Ethylene Carbonate & $\mathrm{C}_{3} \mathrm{H}_{4} \mathrm{O}_{3}$ & 88.06 & 1.32 & 1.00 & 3.33 & 4.40 \\
\hline Diethyl Carbonate & $\mathrm{C}_{5} \mathrm{H}_{10} \mathrm{O}_{3}$ & 118.13 & 0.98 & 1.00 & 3.33 & 3.25 \\
\hline Dimethyl Carbonate & $\mathrm{C}_{3} \mathrm{H}_{6} \mathrm{O}_{3}$ & 90.08 & 1.07 & 1.00 & 3.33 & 3.57 \\
\hline $\begin{array}{l}\text { Lithium } \\
\text { Hexafluorophosphate }\end{array}$ & $\mathrm{LiPF}_{6}$ & 151.91 & 1.55 & & & 1.52 \\
\hline Total Batch Mixture [mL & 10 & & & & & \\
\hline
\end{tabular}

$1 \mathrm{M} \mathrm{LiPF} 6$ in EC+DEC (1:1 vol\%)

\begin{tabular}{|c|c|c|c|c|c|c|}
\hline Chemicals & Formula & $\begin{array}{c}\text { Molecular } \\
\text { Weight } \\
\text { [g/mol] }\end{array}$ & $\begin{array}{l}\text { Density } \\
{[\mathrm{g} / \mathrm{mL}]}\end{array}$ & $\begin{array}{l}\text { Volume } \\
\text { Ratio }\end{array}$ & $\begin{array}{c}\text { Volume } \\
{[\mathrm{mL}]}\end{array}$ & $\begin{array}{c}\text { Amount of } \\
\text { Chemical } \\
\text { [g] }\end{array}$ \\
\hline Ethylene Carbonate & $\mathrm{C}_{3} \mathrm{H}_{4} \mathrm{O}_{3}$ & 88.06 & 1.32 & 1.00 & 5.00 & 6.60 \\
\hline Diethyl Carbonate & $\mathrm{C}_{5} \mathrm{H}_{10} \mathrm{O}_{3}$ & 118.13 & 0.98 & 1.00 & 5.00 & 4.88 \\
\hline $\begin{array}{l}\text { Lithium } \\
\text { Hexafluorophosphate }\end{array}$ & $\mathrm{LiPF}_{6}$ & 151.91 & 1.55 & & & 1.52 \\
\hline
\end{tabular}

Through testing it was determined that a $1 \mathrm{M} \mathrm{LiPF}_{6}$ in EC+DEC (3:7 vol\%) electrolyte system yielded the best results; therefore, it was selected to be utilized throughout the rest of the research.

\subsection{Separator Selection}

The last remaining component within a lithium ion cell is the separator material. The separator is typically a micro-porous plastic polymer and has the essential function of preventing physical contact of the positive and negative electrodes. This prevents electrons from flowing between the electrodes, but still allows an unrestricted flow of ions through it in the liquid electrolyte. This research consisted of evaluating two different separator materials microstructure as they came before being manufactured in a classical lithium ion cell, as well as reevaluating them after the cell was fabricated and subject to cycling at a $1 \mathrm{C}$ cycling rate for 30 cycles.

The separators examined in this research were both $25 \mu \mathrm{m}$ thick films manufactured by a dry stretch process. The first separator film was a tri-layer 
membrane polypropylene/polyethylene/polypropylene (PP/PE/PP) (MTI Corporation) battery separator film and contained $40 \%$ porosity with an average pore size of $0.03 \mu \mathrm{m}$ (Figure 29-A). The other film examined was Celgard's 2325 Tri-layer PP/PE/PP battery film (courtesy of Celgard), which contains a $41 \%$ porosity with a pore size of $0.09 \times 0.04$ $\mu \mathrm{m}$ (Figure 29-B). Even though both of these films are similar in their physical properties, the microstructures greatly vary due to each manufacture's precise dry process fabrication of the film. As evident in the SEM micrographs, the MTI tri-layer separator contains a larger elongated slit pore almost ripped design compared to the Celgards 2325 tri-layer separator, which exhibits a much better formed and slightly elliptical slit pore makeup. The pore properties are specifically dependent upon the uniaxial and biaxial stretching fabrication process of the film [41].
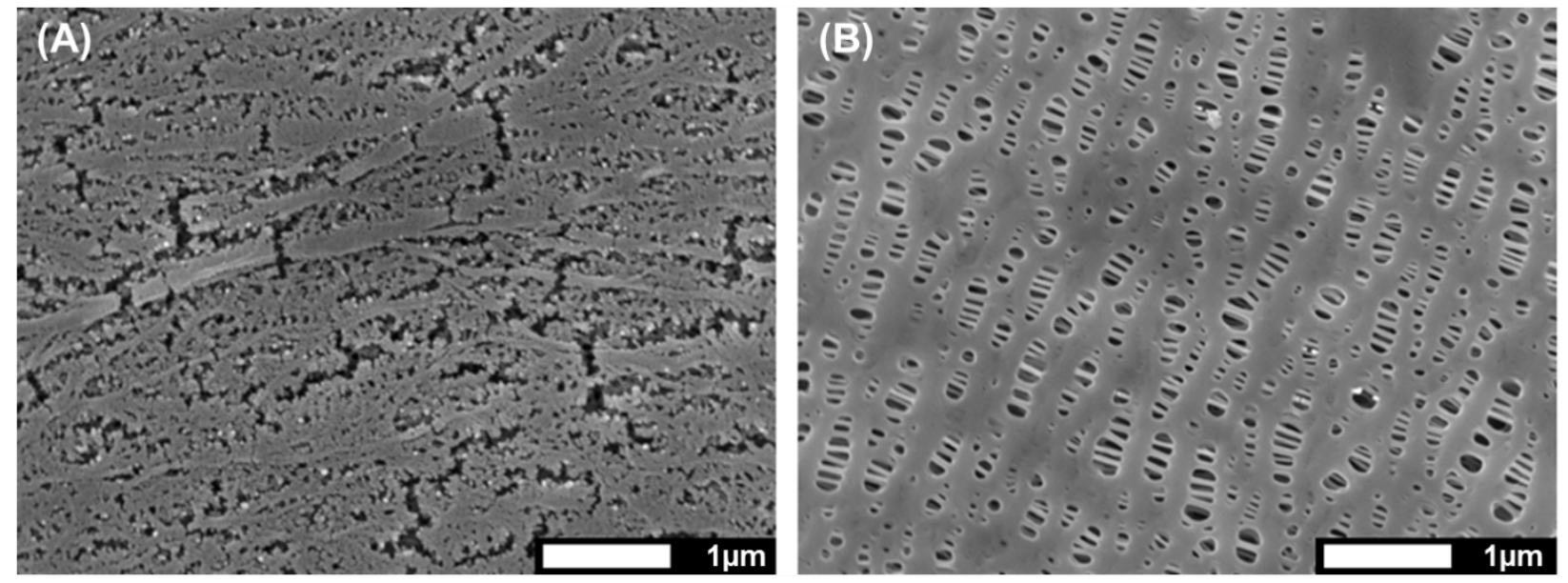

Figure 29 - Micrographs of unused tri-layer PP/PE/PP separators (A) MTI Corporation battery film (B) Celgard 2325 film.

Two significant problems were evident during cycling of the MTI tri-layer film when compared to Celgard's tri-layer film, which are both explained by the micrographs of the cycled cells (Figure 30). Cells manufactured with MTl's separator film exhibited an extremely high irreversible capacity loss as well as a much greater capacity fade throughout cycling. Also, a much higher failure rate was also evident with MTI's separator due to the fabricated cells shorting out. 

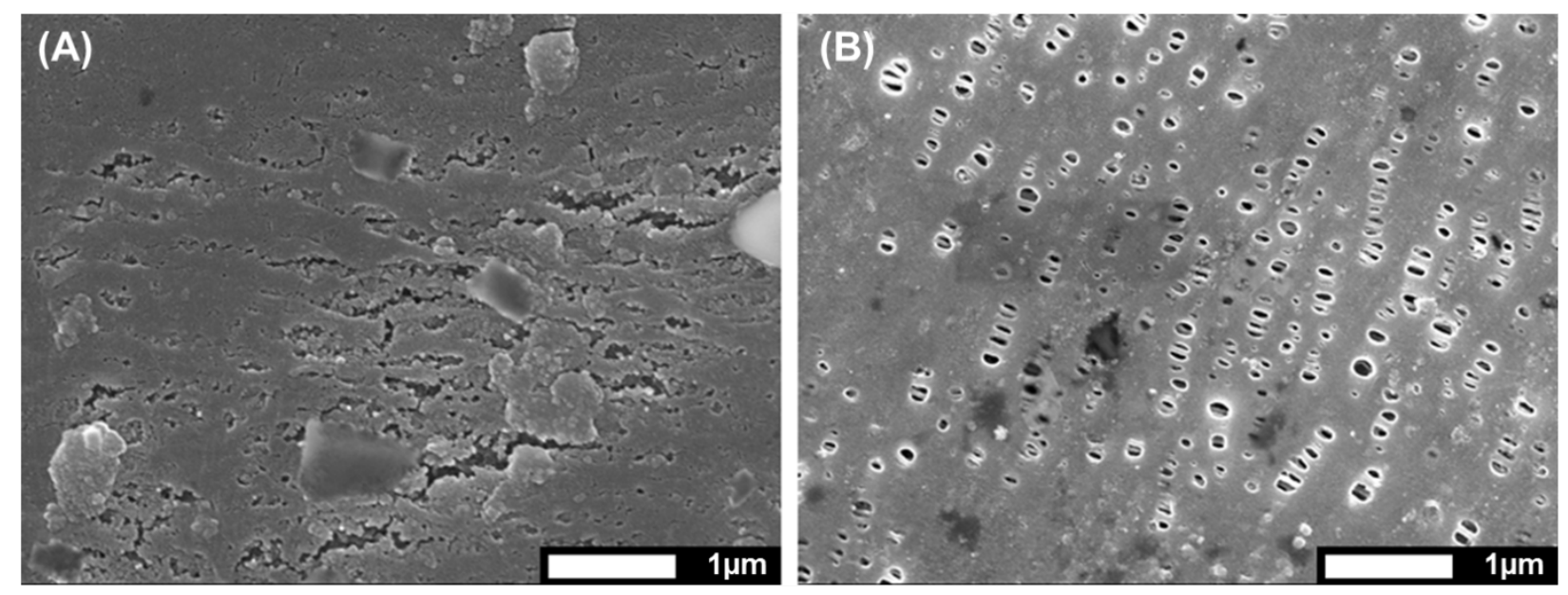

Figure 30 - Micrographs of cycled tri-layer PP/PE/PP separators (A) MTI Corporation battery film (B) Celgard 2325 film.

These encountered problems were due to the pore design of MTI's tri-layer fabricated separator. The larger cracks in the separator can be packed full of particles off of the electrodes, which are gradually filled during cycling causing a continual growth of the SEl layer resulting in a larger capacity fade. Furthermore, the much higher failure rate of the cells was directly due to the larger pores allowing particles from the electrodes to come in contact with each other allowing for a flow of electrons within the cell. This effect is evident when the cell does not charge, which means the pores in the separator allowed an immediate mass flow on internal electrons when the charge process was initiated. This failure effect is also exhibited when the cell encounters an extremely high irreversible capacity loss followed by a rapidly growing capacity fade until failure. Due to these results and superior performance characteristics found throughout literature, Celgard's 2325 tri-layer superiorly manufactured separator film was selected to be utilized for cell fabrication in this research [41].

\subsection{Test Cell Construction}

The initial testing utilized a reusable three electrode split cell (Figure 31); however, in order to test the amount of cells desired for this research it was deemed that another way to manufacture multiple cells for testing would be beneficial. Two different cell constructions were considered: pouch cells (Figure 5) and coin cells (Figure 6). After further research of both types of cells, a CR2032 coin cell base was 
chosen due to its size, availability, ease of manufacturing, and they are the most inexpensive type of battery to fabricate [4]. There are several steps required in order manufacture coin cells with fabricated materials. These steps are cutting of the electrodes, cutting of the separator, cell stacking, electrolyte insertion, pressing of the cells, and extra sealing of the cells. Since the equipment to fabricate these cells is expensive many of the traditional steps have been modified in order to fit the given budget for materials and supplies.

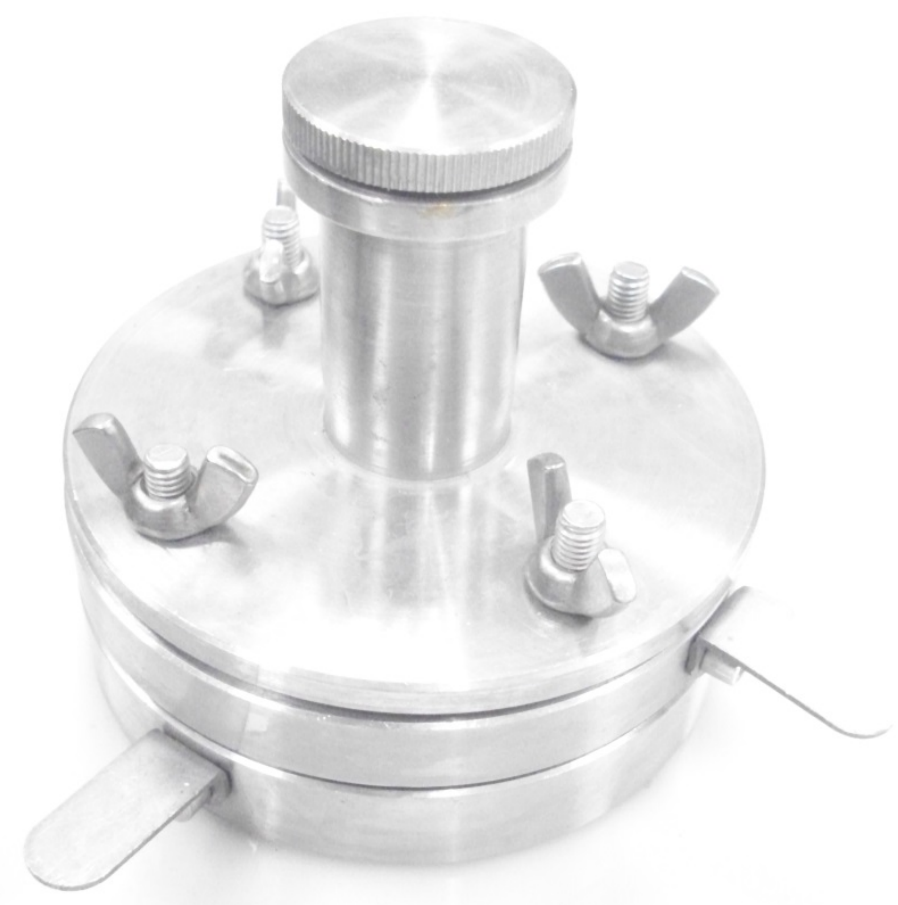

Figure 31 - Three electrode split cell testing apparatus.

The shape of the electrode for a CR2032 coin cell is circular with a $15 \mathrm{~mm}$ diameter, while the separator is slightly larger with an $18 \mathrm{~mm}$ diameter to ensure that the cathode and anode do not touch throughout manufacturing. The most efficient way to cut the electrodes was with a $15 \mathrm{~mm}$ round hole arch punch. The process for this consisted of laying the electrode tape casts over a cutting board and tapping the punch with a hammer to produce a perfect $15 \mathrm{~mm}$ diameter circle for every electrode.

The separator material proved harder to achieve a clean cut with a round hole arch punch, or an X-Acto knife, than the electrodes were. Therefore, another method for 
cutting the separator was necessary. A C-200 carbon dioxide $\left(\mathrm{CO}_{2}\right)$ powered laser cutting system was implemented by programming the shape of the separator into Corel 8's drawing program (Figure 32). The laser cutter easily cut the separator material that resulted in manufacturing multiple separators from a single cycle into perfect circles.

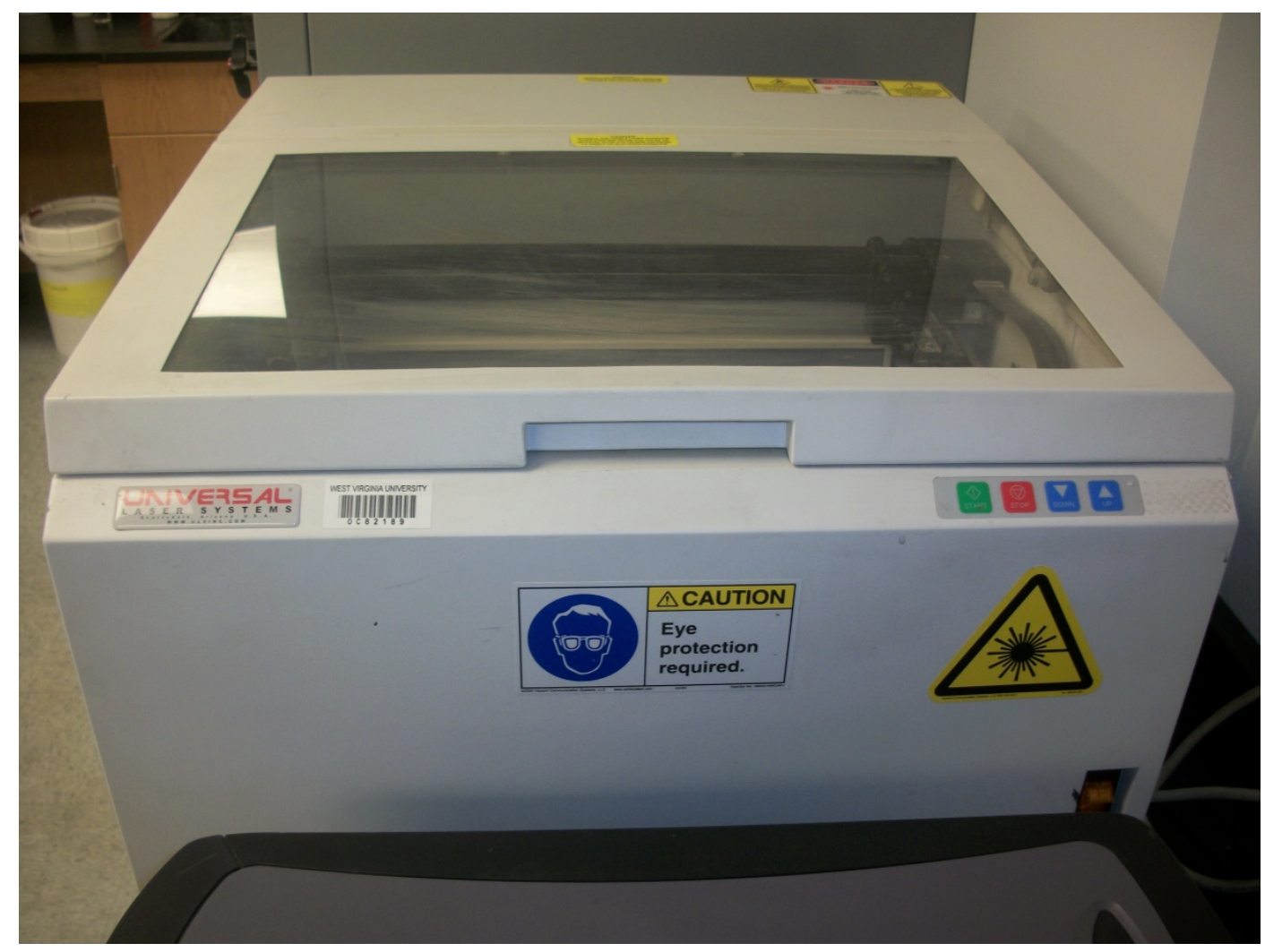

Figure 32 - C-200 carbon dioxide programmable laser cutter system.

After all of the materials were prepared for fabricating a coin cell, the respective cathodes and anodes were examined for deformation, weighed to calculate the theoretical capacitances, labeled, and then transferred into the glovebox. These were accompanied by the coin cell case, spring, spacer, separator, and electrolyte carbonate solution (Figure 33). Once everything had been successfully transferred into the glovebox, it was left alone in the inert atmosphere for at least 24 hours to ensure full removal of any moisture contained in any of the materials. 


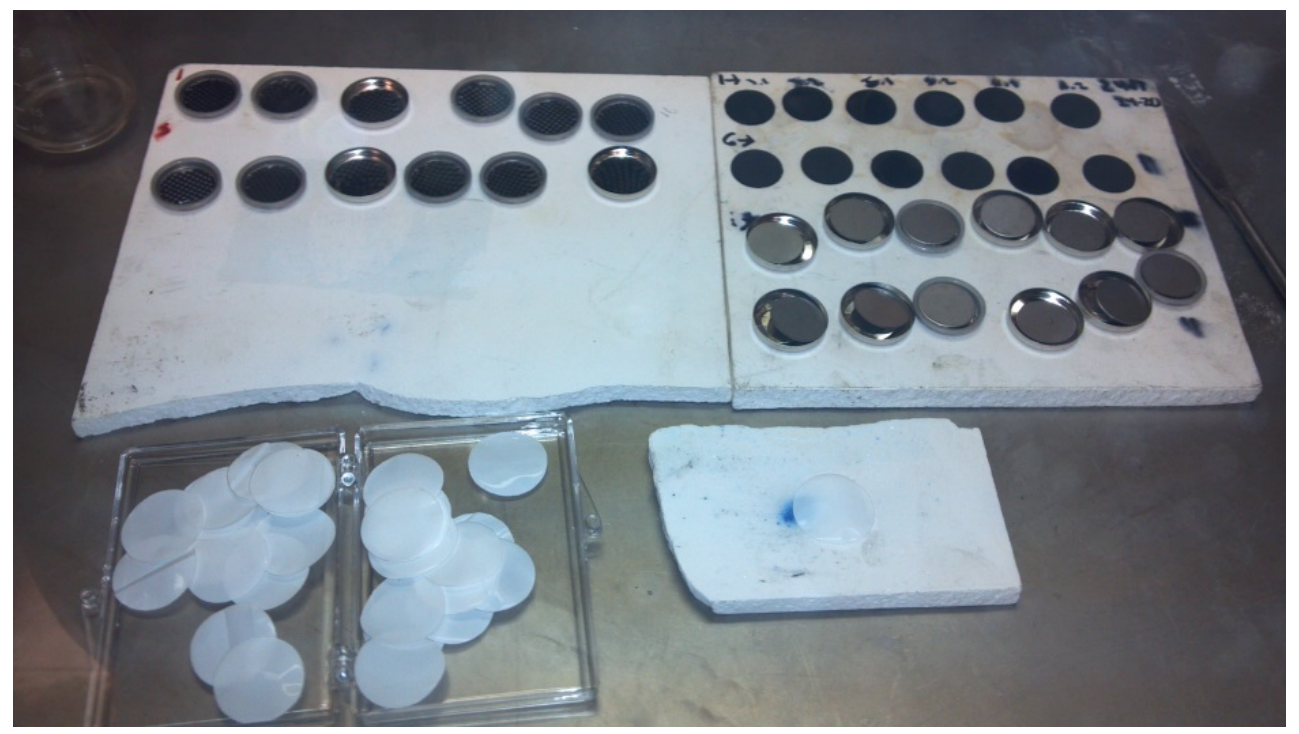

Figure 33 - Picture of coin cell fabrication pieces in glovebox.

The next step in producing a coin cell was to finish synthesizing the electrolyte by adding the lithium salt $\mathrm{LiPF}_{6}$ to the carbonate solution and mixing it with a stir rod on a stir plate for an hour (Figure 34). Once the solution was prepared, the coin cells were ready for fabrication. While initially producing the coin cells, obstacles such as how much electrolyte was required for each cell, how long it took for the electrolyte to permeate through the electrodes, and how to successfully seal each coin cell, were solved through a trial and error process. When too much electrolyte was in the cell, it would cause an overflow during sealing; if not enough electrolyte was added, the electrochemical properties of the cell suffered. Eventually, after carefully documenting each step for several batches of coin cells, a process that yielded a high rate of successful batteries was developed. This exact process was followed for all of the coin cell performance tests since each cell needed to be constructed in the same manner for all of the test cells throughout the research to be comparable to each other. 


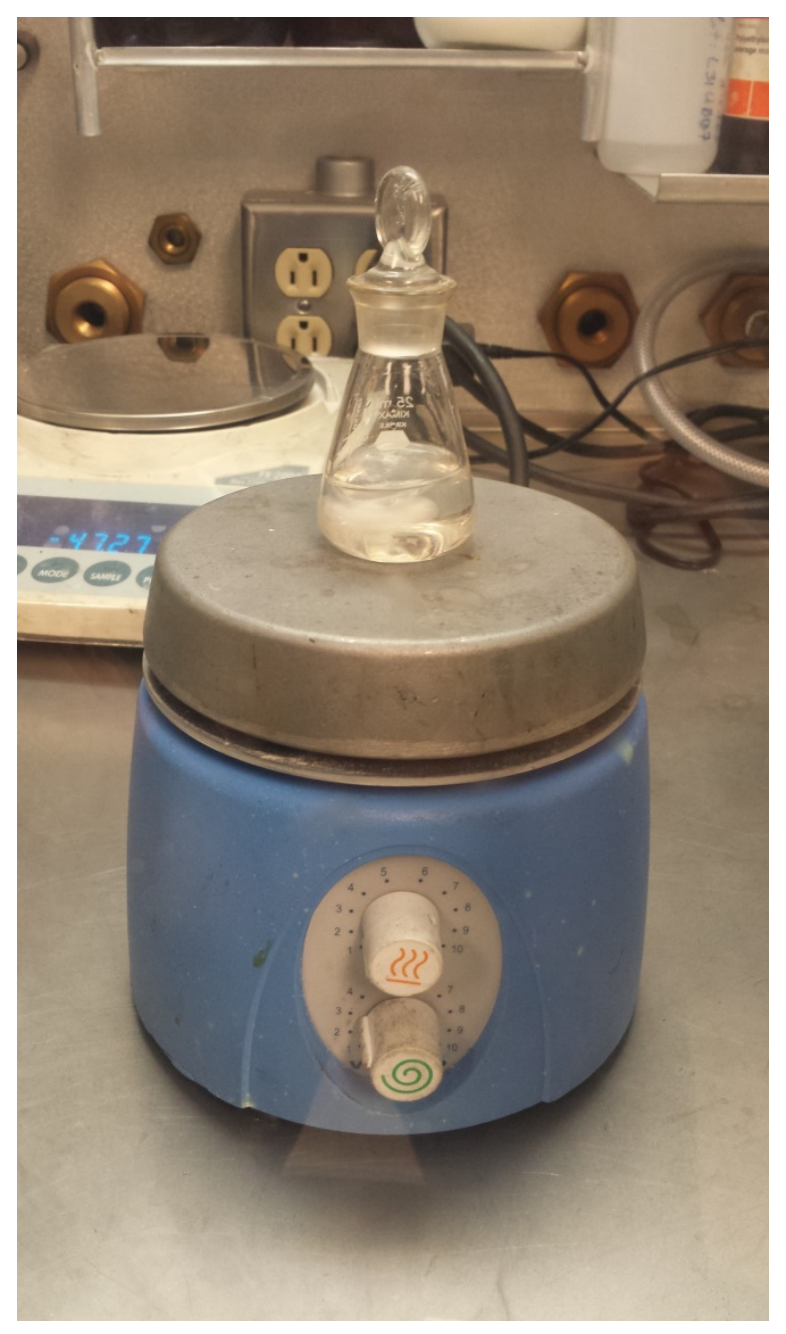

Figure 34 - Electrolyte mixing process conducted in inert atmosphere of glovebox.

The developed process started by adding $10 \mathrm{mg}$ of the electrolyte to each of the electrodes and letting the solution permeate throughout the electrodes for 30 minutes. Next the saturated anode was placed in the negative, smaller side, of the coin cell where a separator was carefully centered and placed on top of the anode along with 5 $\mathrm{mg}$ of more electrolyte solution on top of the separator. Then the saturated cathode was centered and stacked on top of the separator followed by a stainless steel spacer and spring, which was used to ensure that an even pressure was distributed throughout the entire surface of the electrodes to the separator (Figure 35). Finally, the top side of the coin cell was added enabling the coin cell to be sealed by an arbor press with CR2032 dies. The process of crimping the cell for an effective seal proved tedious at first, but by 
carefully centering and slowly crimping each cell a repetitive method was developed (Figure 36).

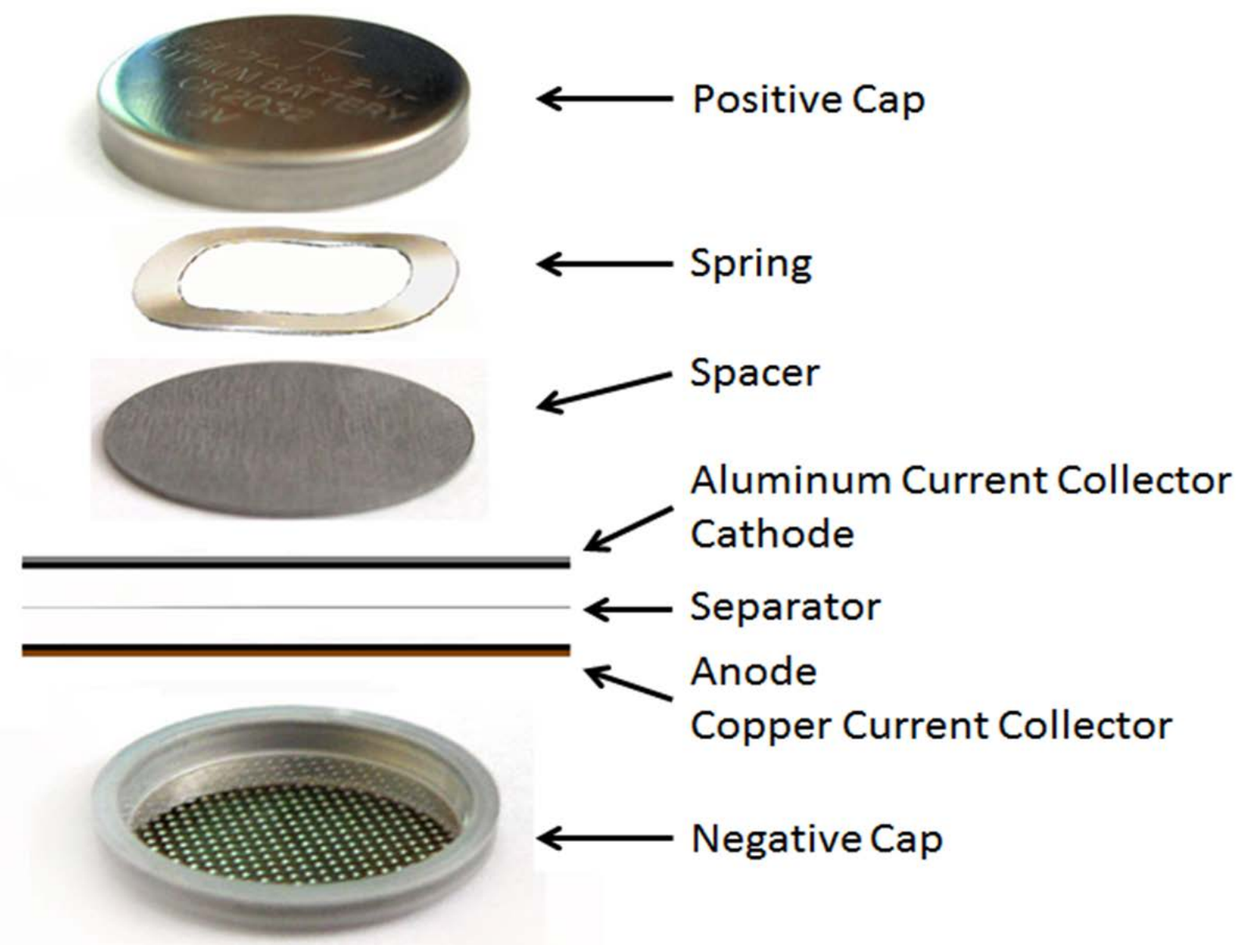

Figure 35 - Schematic of internal layered components comprised in performance test coin cells. 


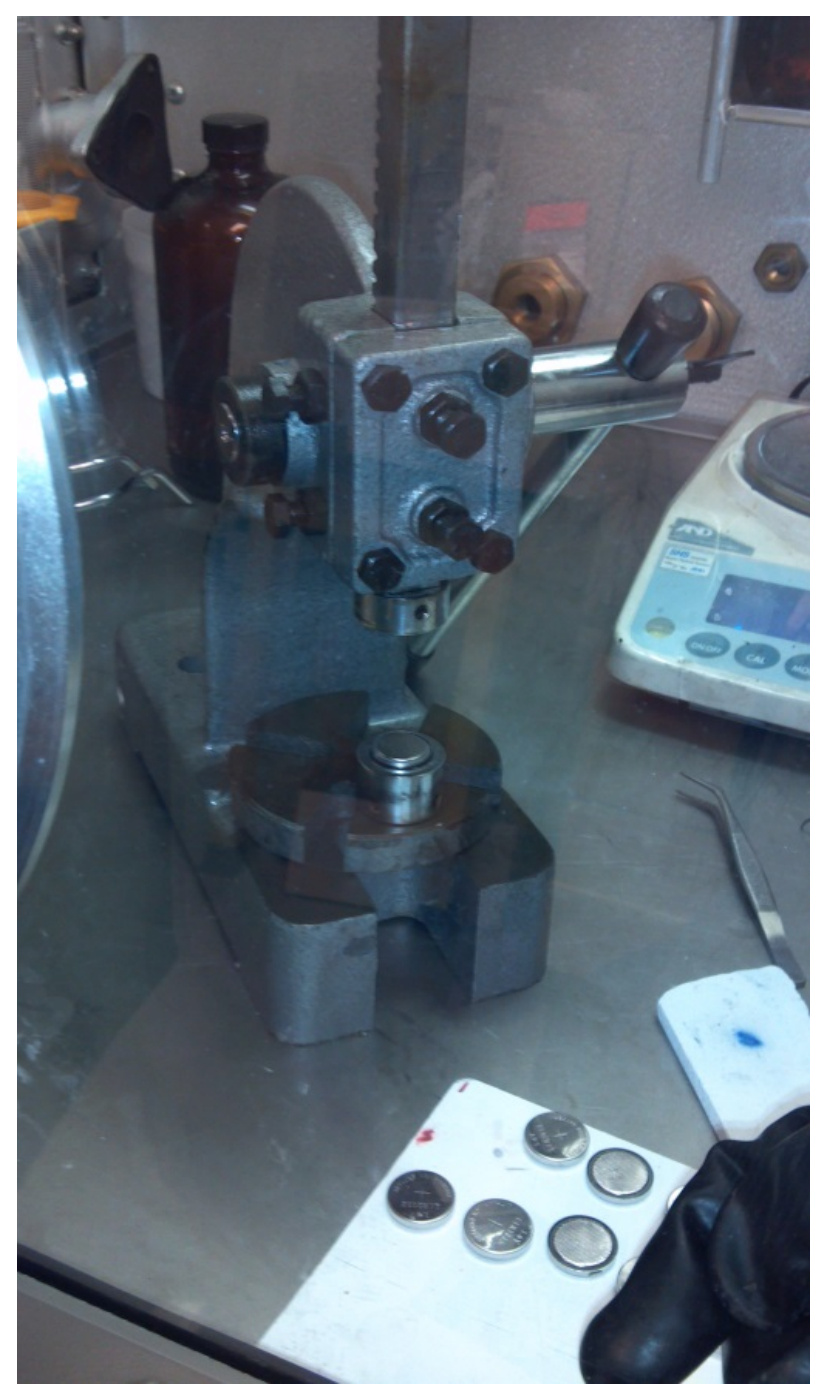

Figure 36 - Battery arbor press with CR2032 coin cell dies, ready to crimp coin cell.

Furthermore, an extra measure to ensure a successful seal was initiated, due to the fact that some of the plastic seals on the purchased coin cell cases produced hairline cracks after pressing into completed cells. As a safety precaution, epoxy was spread around the seal of the coin cells immediately following their removal from the glovebox, eliminating any leaks to develop while cycling (Figure 37). 


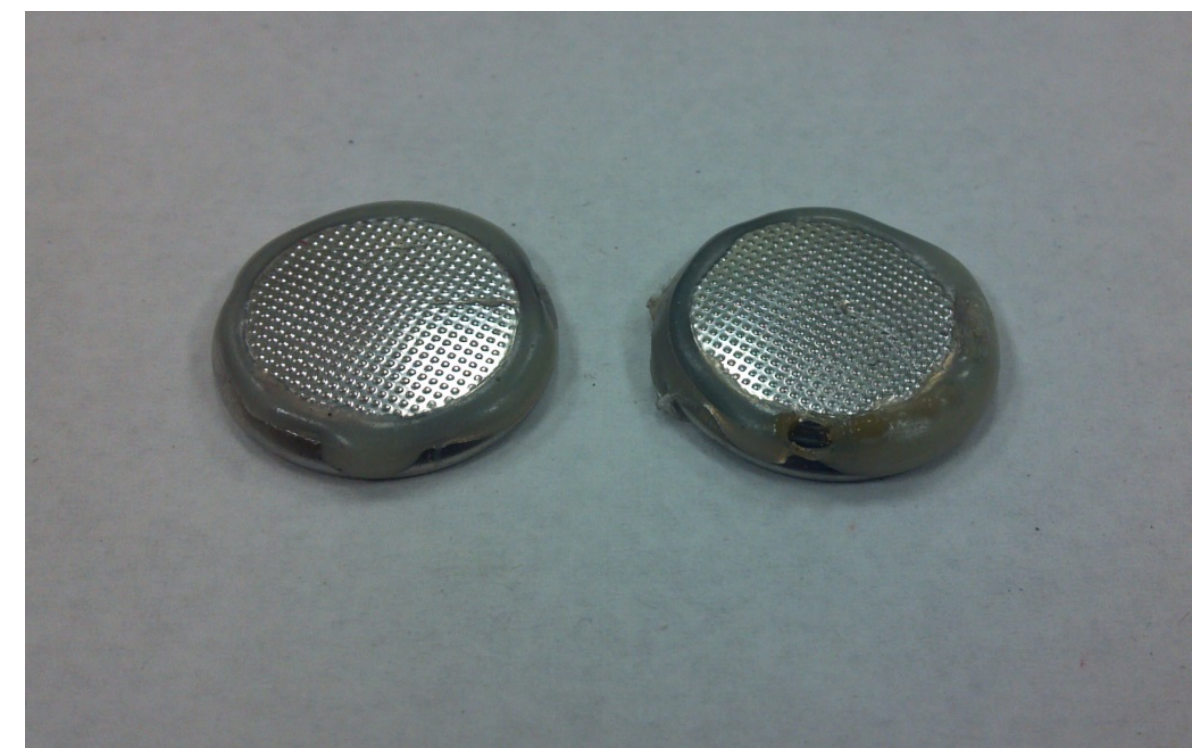

Figure 37 - Successfully epoxy sealed coin cell followed by a failed epoxy sealed coin cell with electrolyte leakage from gaseous buildup.

\subsection{Performance Testing}

After each cell was manufactured, the next phase of testing the cell was initiated. This way comprised of forming the solid electrolyte interface (SEI) layer followed by charging and discharging (cycling) the cell. For each series of performance tests, the specific protocol was slightly tailored and will be stated within each respective section; however, an overall protocol for testing all of these cells is delivered in this section.

For all of the in-house fabricated cells, the theoretical capacitance (C) was calculated based on the mass of the cathode (Table 4). The SEl layer was formed based on the theoretical $C$ by very slowly charging the cell to $4.2 \mathrm{~V}$ then cycling the cell at a constant current (CC) $\mathrm{C} / 25-\mathrm{C} / 40$ rate for 1-3 cycles from 3.0-4.2 $\mathrm{V}$ for a smooth SEI formation layer. 
Table 4 - Example of theoretical and experimental capacity calculations for in-house fabricated coin cells.

\begin{tabular}{|c|c|c|c|c|c|c|c|}
\hline \multicolumn{2}{|c|}{ Specific Capacities: } & \multicolumn{3}{|c|}{$\mathrm{LiCoO}_{2}-140[\mathrm{mAh} / \mathrm{g}]$} & \multicolumn{3}{|c|}{ Graphite - 372 [mAh/g] } \\
\hline Electrolyte & Cathode & $\begin{array}{c}\text { Volume } \\
\mathrm{LiCoO}_{2} \\
{[\%]}\end{array}$ & Anode & $\begin{array}{c}\text { Volume } \\
\text { Graphite } \\
\text { [\%] }\end{array}$ & Separator & $\begin{array}{l}\text { Cathode } \\
\text { Mass [g] }\end{array}$ & $\begin{array}{c}\text { Mass } \\
\text { of } \\
\mathrm{LiCoO}_{2} \\
{[\mathrm{~g}]}\end{array}$ \\
\hline $\begin{array}{l}1 \mathrm{M} \mathrm{LiPF}_{6} \\
\text { EC+DEC } \\
\text { (3:7 vol\%) }\end{array}$ & $\begin{array}{c}\mathrm{LiCoO}_{2} \text { (In- } \\
\text { House) }\end{array}$ & 80.00 & $\begin{array}{l}\text { Graphite } \\
\text { (Alfa Aesar) }\end{array}$ & 90.00 & $\begin{array}{l}\text { Celgrad - } \\
2325\end{array}$ & 0.0822 & 0.0658 \\
\hline
\end{tabular}

\begin{tabular}{|c|c|c|r|r|r|r|r|}
\hline $\begin{array}{c}\text { Anode } \\
\text { Mass [g] }\end{array}$ & $\begin{array}{c}\text { Theoretical } \\
\text { Capacity } \\
\text { Cathode } \\
\text { [mAh] }\end{array}$ & $\begin{array}{c}\text { Cathode } \\
\text { C/25 } \\
{[\mathrm{mAh} / \mathrm{g}]}\end{array}$ & $\begin{array}{c}\text { SEl Layer } \\
\text { Cycles }\end{array}$ & $\begin{array}{c}\text { Real } \\
\text { Discharge } \\
\text { Capacity } \\
\text { [mAh] }\end{array}$ & $\begin{array}{c}\text { Rate: } \\
\text { C/2 }\end{array}$ & $\begin{array}{c}\text { Rate: } \\
\text { C/5 }\end{array}$ & $\begin{array}{c}\text { Rate: } \\
\text { C/20 }\end{array}$ \\
\hline 0.0484 & 9.2064 & 0.368 & 1.5 & 8.253 & 4.127 & 1.651 & 0.413 \\
\hline
\end{tabular}

Once the formation cycles were completed, the coin cells were charged at a constant current $(\mathrm{CC}) \mathrm{C} / 5$ rate, then held at a constant voltage (CV) $4.2 \mathrm{~V}$ until the current dropped below $\mathrm{C} / 25$ or a maximum of two hours had passed. A rest period of 15 minutes was taken after the cell was charged to allow for the voltage to stabilize. Following the rest period, each cell was then discharged to $3.0 \mathrm{~V}$ at a (CC) $2 \mathrm{C}-\mathrm{C} / 5$ rate, where another 15 minute rest period was taken. Each cell was then cycled multiple times following a direct protocol for each series of performance tests.

Two test stands were utilized for cell performance testing. The initial test stand consisted of separate units for cycling. Charging was performed by a BK Precision 9121A programmable DC power supply, while discharging was performed with a BK Precision 8500 programmable DC electronic load (Figure 38). This test stand proved that functional cells could be produced in the lab with the three electrode split cell. However, with this apparatus only a single cell could be examined at a time and each unit had to be programmed and started by a user for each part of cycling. This method required too much time in order to test the amount of cells desired for the research and was only utilized for the split cell testing. 


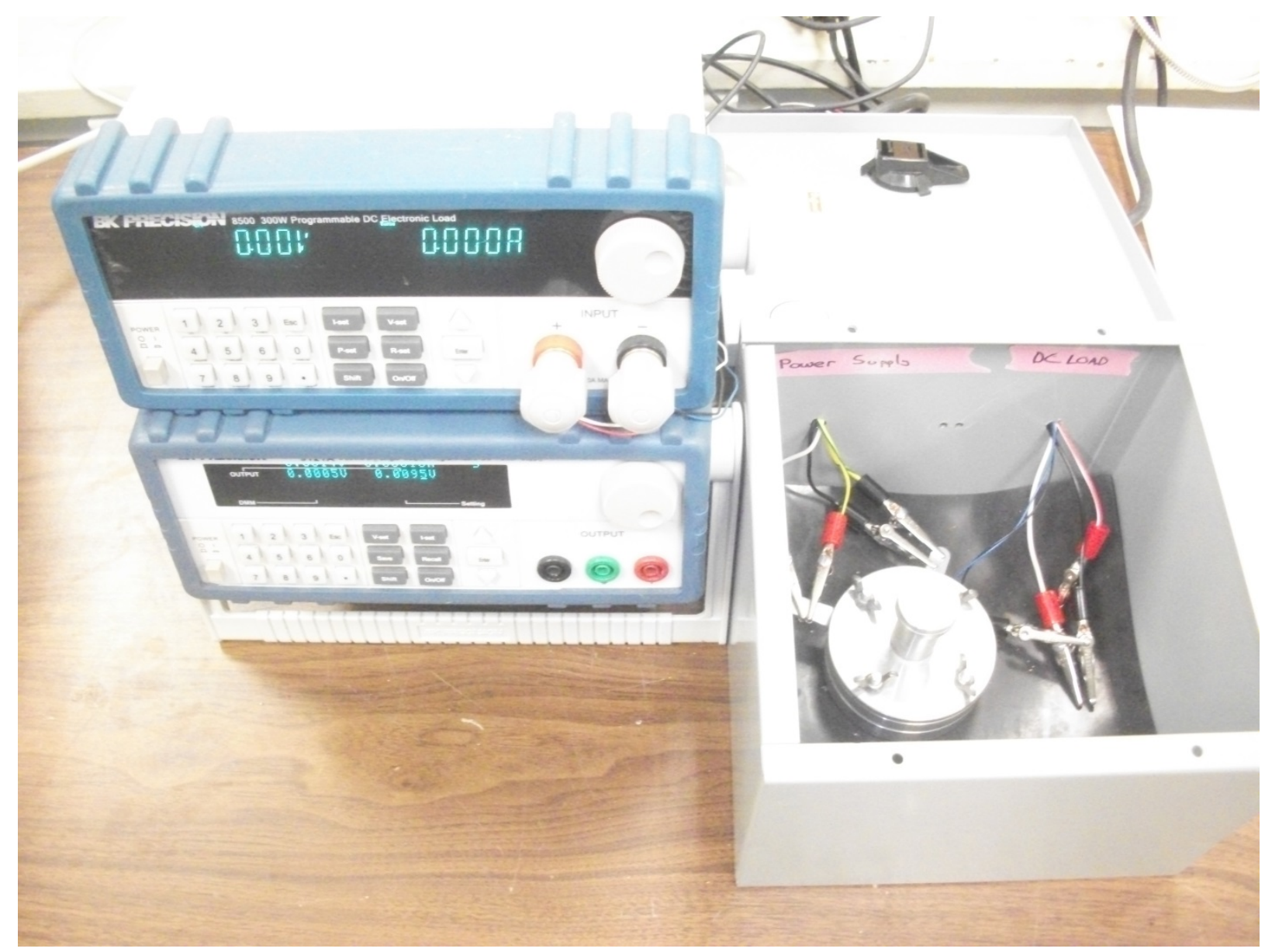

Figure 38 - Initial battery test stand consisting of BK Precision's 9121A programmable DC power supply and 8500 programmable DC electronic load cycling a three electrode split cell in a battery testing box.

The other test stand used for the majority of the testing was MTI's 8 channel battery analyzer. This apparatus easily allowed for 8 separate cycling programs to operate independently since each channel consisted of its own CC and CV source (Figure 39). Each cell could be set up with a specific test protocol programmed with a computer, and then tested simultaneously for as many cycles as desired without further user input. Data was collected in real time and exported to excel when the cell finished its run. This test stand was utilized for all of the coin cell testing. 


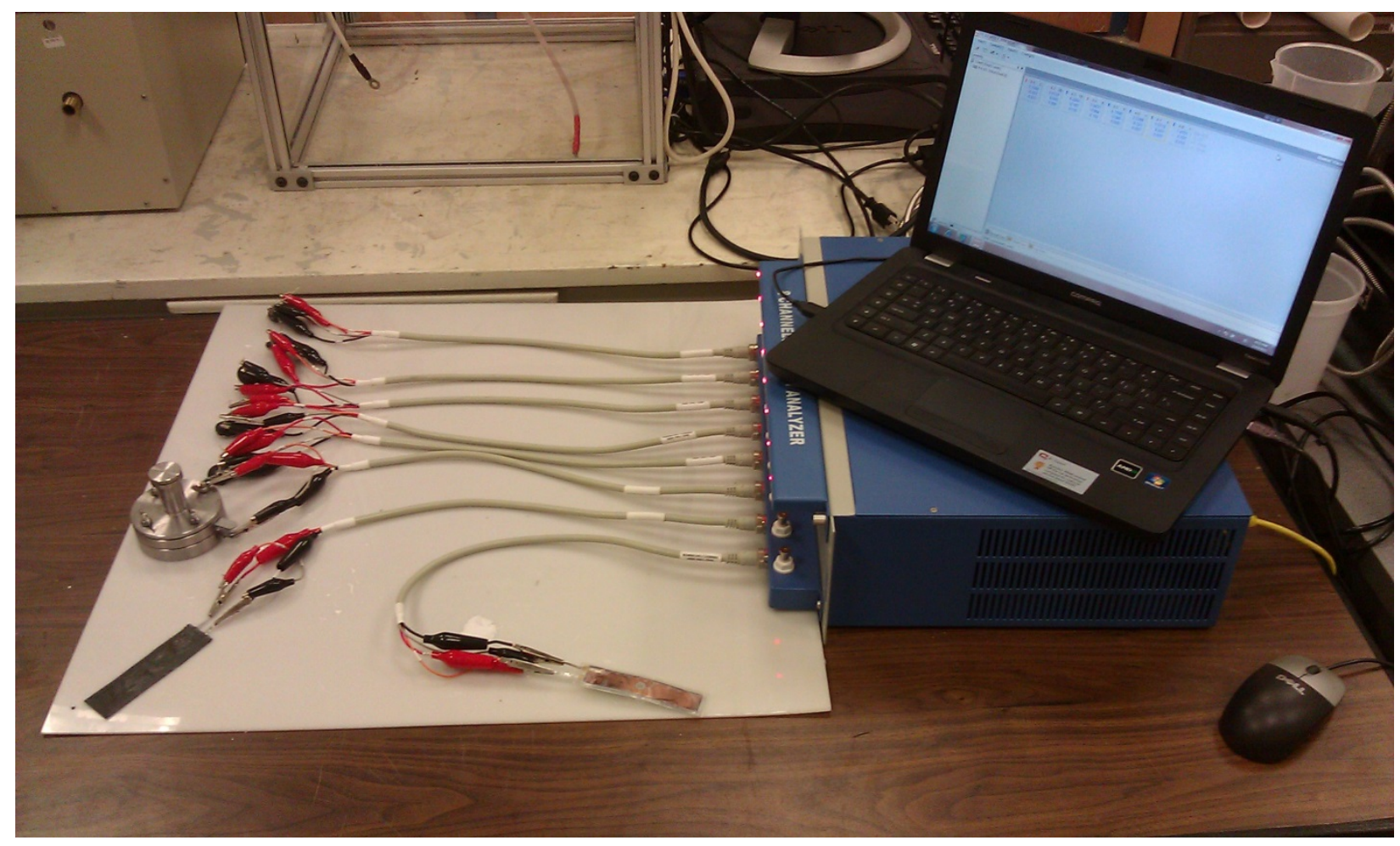

Figure 39 - MTI Corporation 8 channel battery analyzer testing stand setup.

\subsubsection{Split Cell}

Split cell performance tests were conducted for proof of concept of the research. These tests were performed with the initial test stand and proved that an in-house battery could be manufactured, cycled, and the data recorded. Furthermore, cells containing composite materials of carbon fiber as the anode along with fiberglass as the separator were also successfully fabricated and tested. Although the performance of these initial split cell tests were much lower than future manufactured cells, this testing provided the essential beginning steps to fabricating in-house batteries.

The protocol for these cells was later considered naive, because testing was conducted early in the research a true knowledge of how to form and test fabricated lithium ion cells had not been fully established. The electrolyte solution for these series of cells was $1 \mathrm{M} \mathrm{LiPF}_{6}$ in EC+DEC+DMC+PC (4:3:2:1 vol\%), each cell endured 3 cycles with a charge rate of $0.5 \mathrm{C}(1-10 \mathrm{~mA})$ to $4.2 \mathrm{~V}$ and a discharge rate of $0.2 \mathrm{C}(0.01-0.1$ $\mathrm{mA})$ to $2 \mathrm{~V}$. 


\section{Baseline Cell Performance}

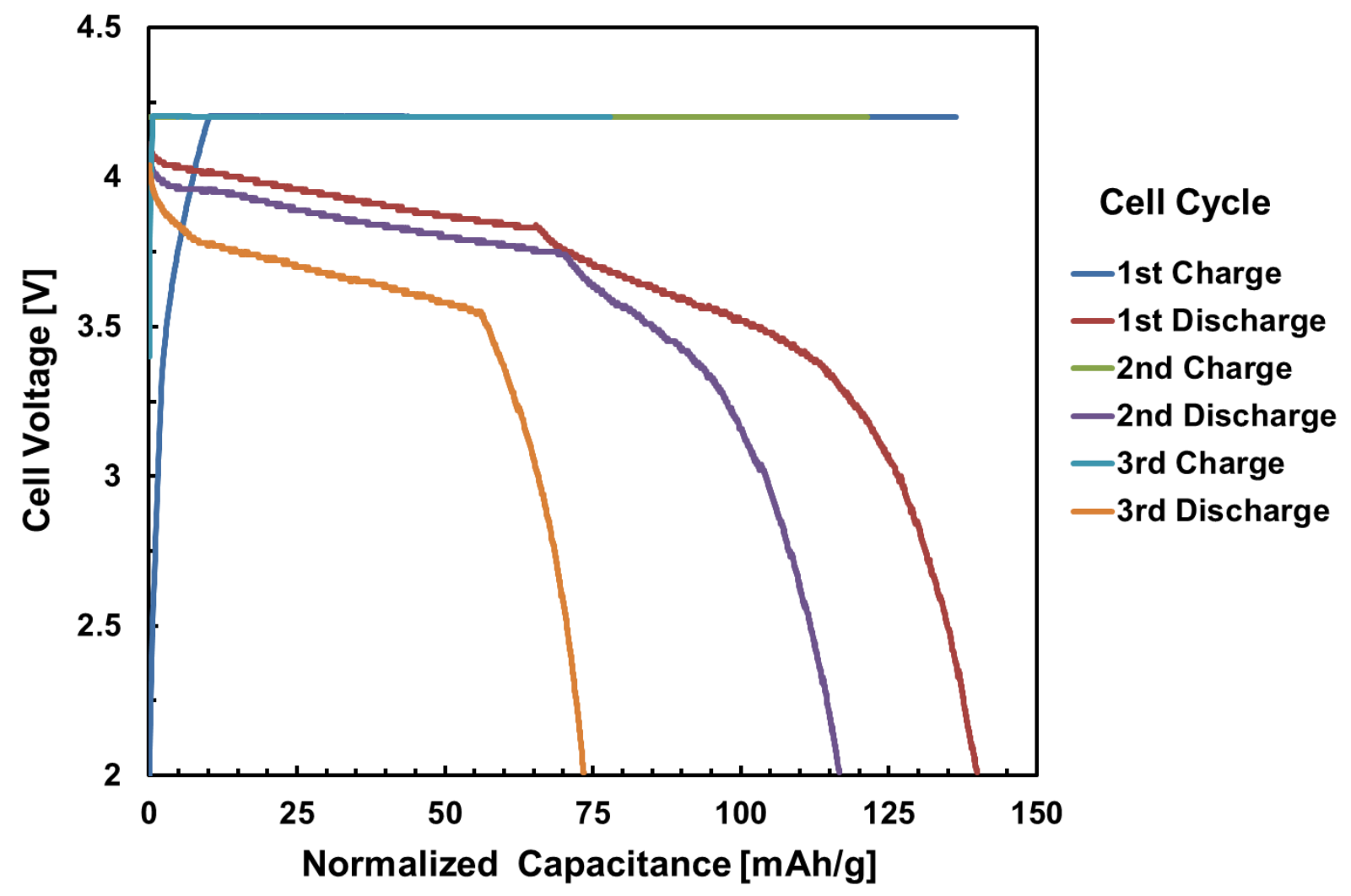

Figure 40 - Baseline performance cycle of classical lithium ion architecture in a three electrode split cell.

This classical lithium ion cell was the first functioning battery achieved with quantitative data collected (Figure 40). This proved that a lithium ion cell could be manufactured and successfully tested for this research. However, data from this series of tests unveiled several problems with the current procedures, such as: 1) overcharging, 2) discharging to a lower voltage than $\mathrm{LiCoO}_{2}$ can recover from without creating a higher irreversible capacity loss, and 3) the formation of a poor SEI layer. These issues are corrected throughout the rest of the performance testing (Section 3.11). Since each three electrode split cell fabricated was subject to the same testing protocol as the baseline, each cell fabricated performed similarly but with much larger irreversible capacity losses. Furthermore, due to the particulate derived anode mixture, the fiberglass replaced separator continually shorted due to movement of the anode powders through the fiberglass porosity. The initial performance for substituting different 
carbon fibers as the anode and fiberglass as the separator are further displayed (Appendix-A).

\subsubsection{Electrolyte}

Once a functional series of tests were completed with the split cell apparatus proving that the in-house fabricated materials could produce a functioning cell, several coin cells were manufactured and tested in the same manner with the 8 channel battery analyzer. However, the cells performed extremely poor and the majority failed. It was realized that the $1 M \mathrm{LiPF}_{6}$ in $E C+D E C+D M C+P C$ (4:3:2:1 vol\%) electrolyte solution was not fully compatible with the graphite used in the classical lithium ion cells and caused them to only function at low cycling rates. This is because PC reacted with the graphite during the charging process causing exfoliation of the graphite anode which resulted in a charge plateau below 4.2 volts [39].

It was determined that a different electrolyte system was required to produce functioning classical lithium ion cells, so three common systems found throughout literature $1 \mathrm{M} \mathrm{LiPF} 6$ in EC+DEC (3:7 vol\%), 1M LiPF 6 in EC+DEC (1:1 vol\%), and 1M $\mathrm{LiPF}_{6}$ in EC+DEC+DMC (1:1:1 vol\%), were simultaneously tested and compared (Figure 41). 


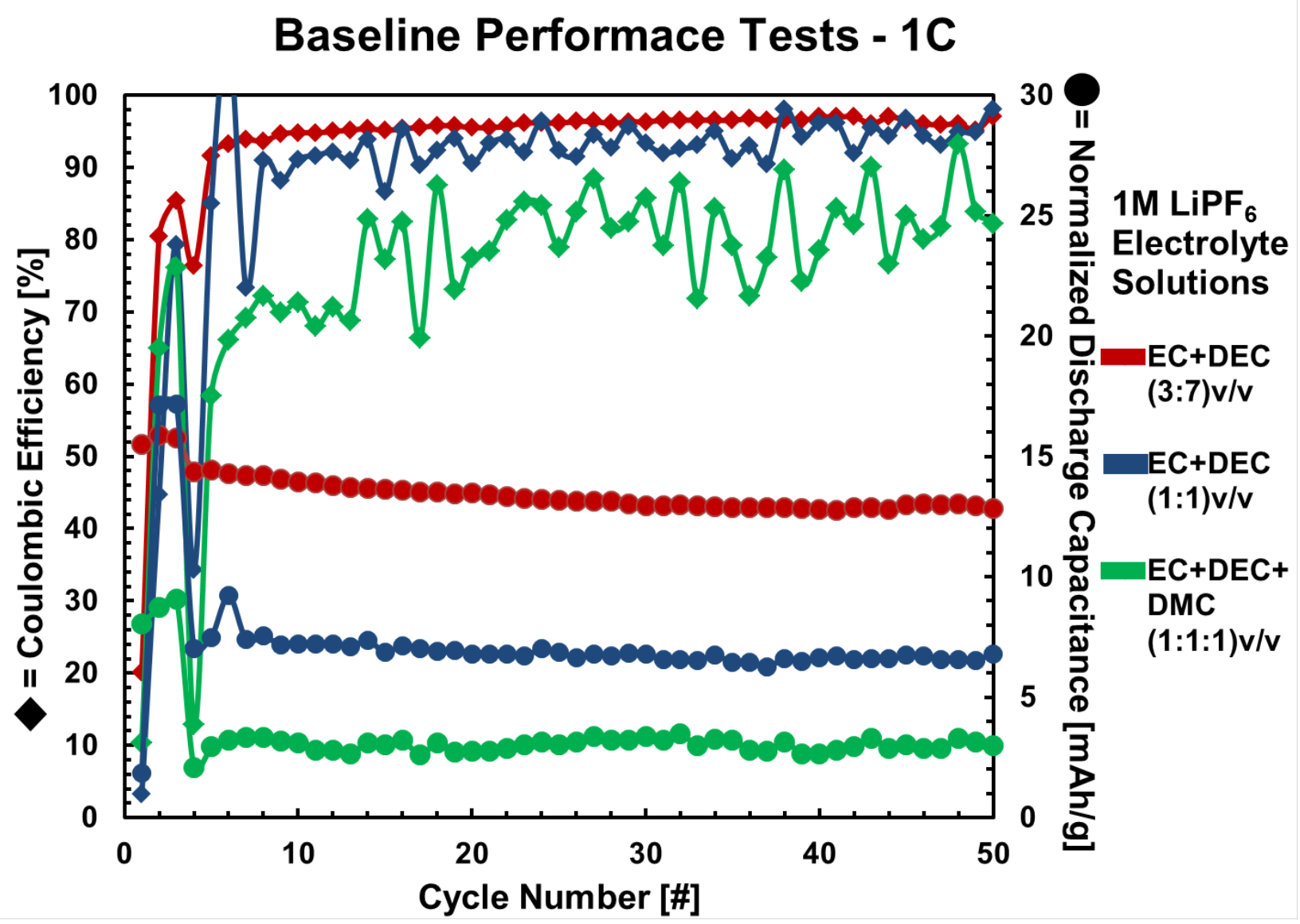

Figure 41 - Electrolyte performance data consisting of the coulombic efficiency overlaid with the normalized discharge capacitance of each electrolyte.

The testing protocol for this series consisted of a C/20 SEI 1 cycle formation rate, followed by a $1 \mathrm{C}$ discharge rate, and a $\mathrm{C} / 2$ charge rate for 50 cycles. Three cells of each of the respective electrolytes were fabricated and tested with the best resulting performance from each solution associated.

The $1 \mathrm{M} \mathrm{LiPF}_{6}$ in EC+DEC (3:7 vol\%) electrolyte solution functioned better compared to the other solutions in the classical lithium ion chemistry of the cells being tested. The coulombic efficiency derived from this electrolyte was the most consistent of the three and showed a typical capacitance fade over cycling, which is main reason it was selected to go forward with in future testing. The normalized discharge capacitance was lower than desired; however, future series of performance tests were completed to increase the capacitance of the fabricated cells. 


\subsubsection{Preliminary Composite Performance Data}

Once a stable electrolyte was selected and additional practice with forming and cycling in-house manufactured lithium ion cells had been conducted, the next series of tests were performed to improve the initial performance data from the composite cells (Section 3.11.1, Appendix-A). With the addition of a more reliable test stand, and the transition to fabricating coin cells, it was important to re-establish and determine how the performance obtained by substituting carbon fiber as the anode and fiberglass as the separator material compared to a classical lithium ion cells' abilities.

The testing protocol for this series of cells included a formation charge rate of $\mathrm{C} / 20$ for 3 cycles followed by a cycling rate of $\mathrm{C} / 2$ for 20 cycles. In order to evenly compare the performance data from the electrochemical tests by each of the cells, the coulombic efficiency and normalized capacitance of the discharge per cycle were examined (Figure 42). 


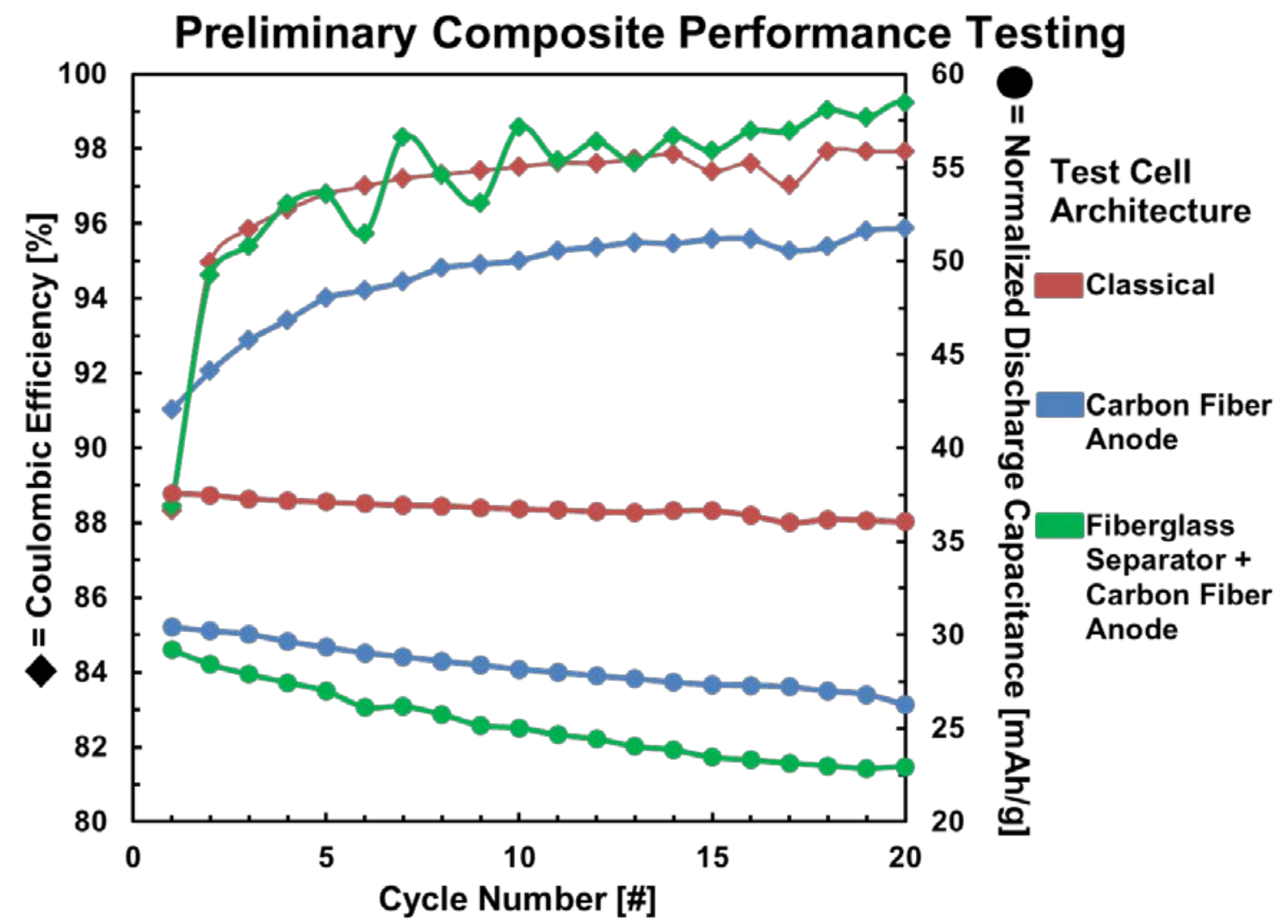

Figure 42 - Electrochemical testing data with different architectures including a novel lithium ion cell, a substituted carbon fiber anode cell, and a substituted fiberglass separator with carbon fiber cell.

This novel cell architecture provided a baseline that consisted of an in-house solid-state synthesized $\mathrm{LiCoO}_{2}$ powder + carbon black + carbon nanofiber + PVDF cathode and corresponding synthetic graphite + PVDF anode. The baseline cell throughout cycling retained a $98 \%$ coulombic efficiency, and displayed roughly a $3 \%$ capacitance fade after 20 cycles.

The first composite comparison was made was by replacing the traditional graphitic anode with a PAN-based $1 \mathrm{~K}$ plain weave ultra-light carbon fiber fabric (Fibre Glast Developments Corporation). No pretreatment of the sample before testing was completed and a copper current collector was still utilized. All of the other components remained the same to achieve an equal comparison. The electrochemical testing with substituted carbon fiber anode resulted in a noticeable decrease in performance. The cell was able to retain a $96 \%$ coulombic efficiency throughout cycling; however, the 
initial irreversible capacity loss was $20 \%$ higher than the baseline and experienced a relative capacitance loss of $15 \%$ due to cycling.

Once the performance of the carbon fiber fabric was established with the Celgard 2325 tri-layer separator, an E-Glass $8 \mathrm{H}$ satin weave fiberglass fabric (Fibre Glast Developments Corporation) was used as a replacement separator within a battery using the carbon weave as the anode. The carbon weave was used in place of a typical particulate derived anode mixture since initial battery tests showed continued shorting due to movement of the anode powders through the fiberglass porosity. The fiber glass weave possessed large diameter pores through its structure that allowed for graphitic powder to migrate and percolate through the structure leading to short circuiting of the cell. The substitution of fiberglass for the tri-layer separator created a dual composite multifunctional battery. The overall trend of this test cell exhibited similar traits to that of the previous cell. The cell exhibited over $98 \%$ coulombic efficiency, but showed $25 \%$ more irreversible capacity loss than the baseline, with a further $23 \%$ loss in capacitance over the rest of testing.

The thickness from the fiberglass fabric led to an unstable coulombic efficiency that caused longer cycling times than originally predicted. The fiberglass fabric had an average thickness of $150 \mu \mathrm{m}$, compared to the $25 \mu \mathrm{m}$ thickness of the Celgard PP/PE separator. This results in a larger ion diffusion path, and thus, an increased overall internal resistance for the battery cell. This resistance caused the composite materials to exhibit a higher irreversible capacity in addition to a larger capacitance loss from cycling than compared to the baseline. The evaluated results of the electrochemical performance data better asserted that fiberglass and carbon fiber are viable alternative battery materials. This evaluation laid out the important beginning steps in the fabrication of a functional composite multifunctional lithium ion battery; however, more steps were required in order to produce in-house, highly functional lithium ion batteries before multifunctional manufactured cell testing could begin.

\subsubsection{Cast Thickness/Anode Material}

Once a stable functioning electrolyte was synthesized, the next course of action was to achieve a higher cell capacitance. Since it was believed that a highly functional 
$\mathrm{LiCoO}_{2}$ cathode material had been fabricated, the focus shifted to improving the electrodes. To solve this problem, the first action was to tape cast the electrodes at larger thicknesses, with the desire to elevate the amount of active lithium particles and raise the capacity of the test cells. The next course of action was to incorporate carbon nanofiber, along with carbon black in the cathode, and eventually the anode. This incorporation was conducted with the purpose to increase the electrical conductivity and active surface area, form a dense electrode system, and include a nanoparticle structural reinforcement component to assist the PVDF binder. Lastly, since all of the testing to this point had been conducted with a synthetic graphite anode, natural graphite was selected for the anode and ran in parallel (Section 3.5.1). The cycling protocol for this series of tests comprised of a C/20 charge to 4.2 followed by a C/20 discharge to determine the actual cell's capacitance, then 30 cycles were completed with each cell at a $1 \mathrm{C}$ rate.

The logic behind casting thicker electrodes was to increase the amount of active material $\left(\mathrm{LiCoO}_{2}\right)$ in the cathode. This was due to the fact that the electrodes were initially cast at $0.3 \mathrm{~mm}$, but due to the low solids loading (10-20\%) in the synthesized inks, the dried electrode tape was $0.01 \mathrm{~mm}$ thick. This resulted in a less than desirable amount of active lithium particles that attributed to the capacitance of the cell. While keeping the thickness of the cast for both cathode and anode the same, the cast thickness was raised to $0.4 \mathrm{~mm}$. This technique improved the normalized capacitance, but only slightly, which led to conducting several more cast thicknesses (Table 5). Each cast thickness continued to increase the overall cell's capacitance as expected (Figure 43); however, as the cast thickness increased the electrode became more fragile and eventually unusable at a cast of $1.25 \mathrm{~mm}$ due to cracking and its brittle nature. 
Table 5 - Tape cast electrode deposition thickness with resulting thickness of electrodes after drying process.

\begin{tabular}{|r|r|r|}
\hline $\begin{array}{l}\text { Wet Tape Cast } \\
\text { Thickness } \\
\text { [mm] }\end{array}$ & $\begin{array}{l}\text { Dry Cathode } \\
\text { Thickness } \\
\text { [mm] }\end{array}$ & $\begin{array}{l}\text { Dry Anode } \\
\text { Thickness } \\
\text { [mm] }\end{array}$ \\
\hline 0.3 & 0.01 & 0.04 \\
\hline 0.4 & 0.02 & 0.06 \\
\hline 0.75 & 0.08 & 0.12 \\
\hline 1 & 0.16 & 0.24 \\
\hline 1.25 & 0.2 & 0.3 \\
\hline
\end{tabular}

\section{Initial Cell Capacitance}

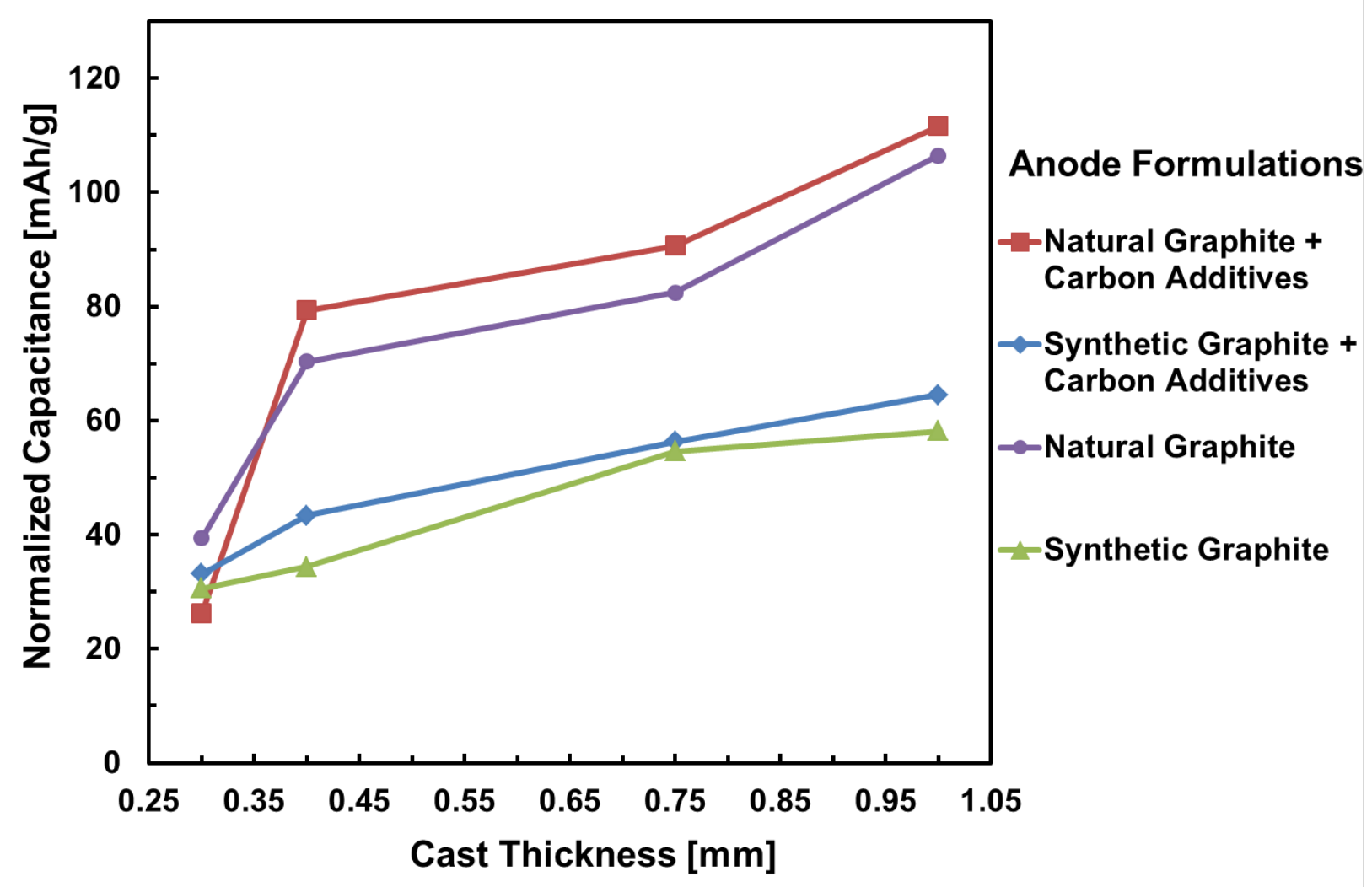

Figure 43 - Normalized initial coin cell capacitance comparing different anode formulations over wet tape casting thicknesses.

The carbon nanofiber material was selected due to its multifunctional properties, which aided the carbon black in improving the electrical conductivity of the cathode electrode. Furthermore, it supported the PVDF binder by incorporating a nanoparticle structural reinforcement component to the electrodes by binding all of the particles 
together (Figure 44). This addition resulted in an increased electrical conductivity magnitude of $10 \mathrm{~S} / \mathrm{cm}$ throughout the cathode, compared to what was achieved by carbon black alone. Improved structural properties of the multifunctional electrode system were evident, especially at the larger cast thicknesses, by significantly reducing the brittleness and flaking of the now dense electrode throughout the manufacturing of test cells.
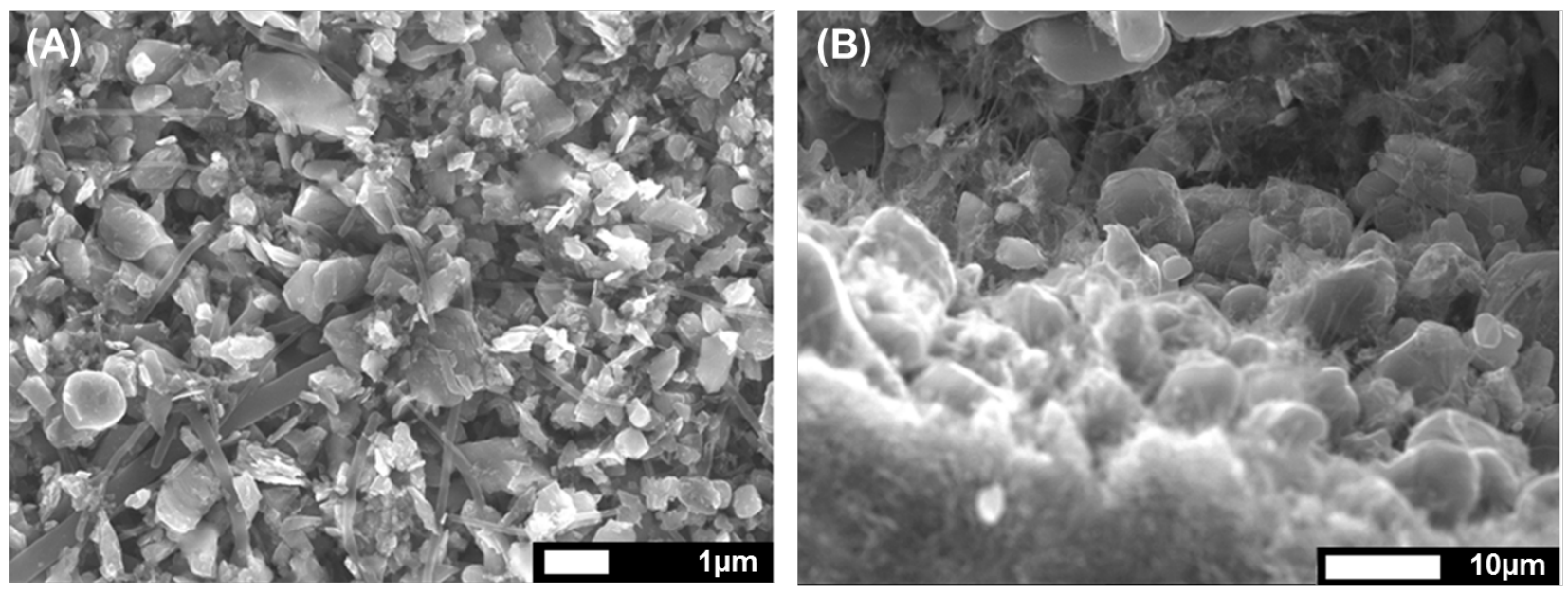

Figure 44 - SEM micrographs of the composite structural electrode, including conductive additives of carbon black and carbon nanofibers as a structural reinforcement (A) cathode electrode and (B) structural composite cathode manually fractured to illustrate carbon nanofiber's structural reinforcement.

The improved structural integrity observed by incorporating carbon nanofiber along with the carbon black in the cathode led to implementation of these particles in the anode (Figure 45). The desired result was a thick and dense electrode system, with improved structural properties due to the nanofiber reinforcement. This addition proved to be highly beneficial once the testing was completed. By splitting 8 vol\% of the graphite material with 4 vol\% carbon black and 4 vol\% carbon nanofiber, an average of $5 \mathrm{mAh} / \mathrm{g}$ increase in the cell's initial capacitance was observed. 

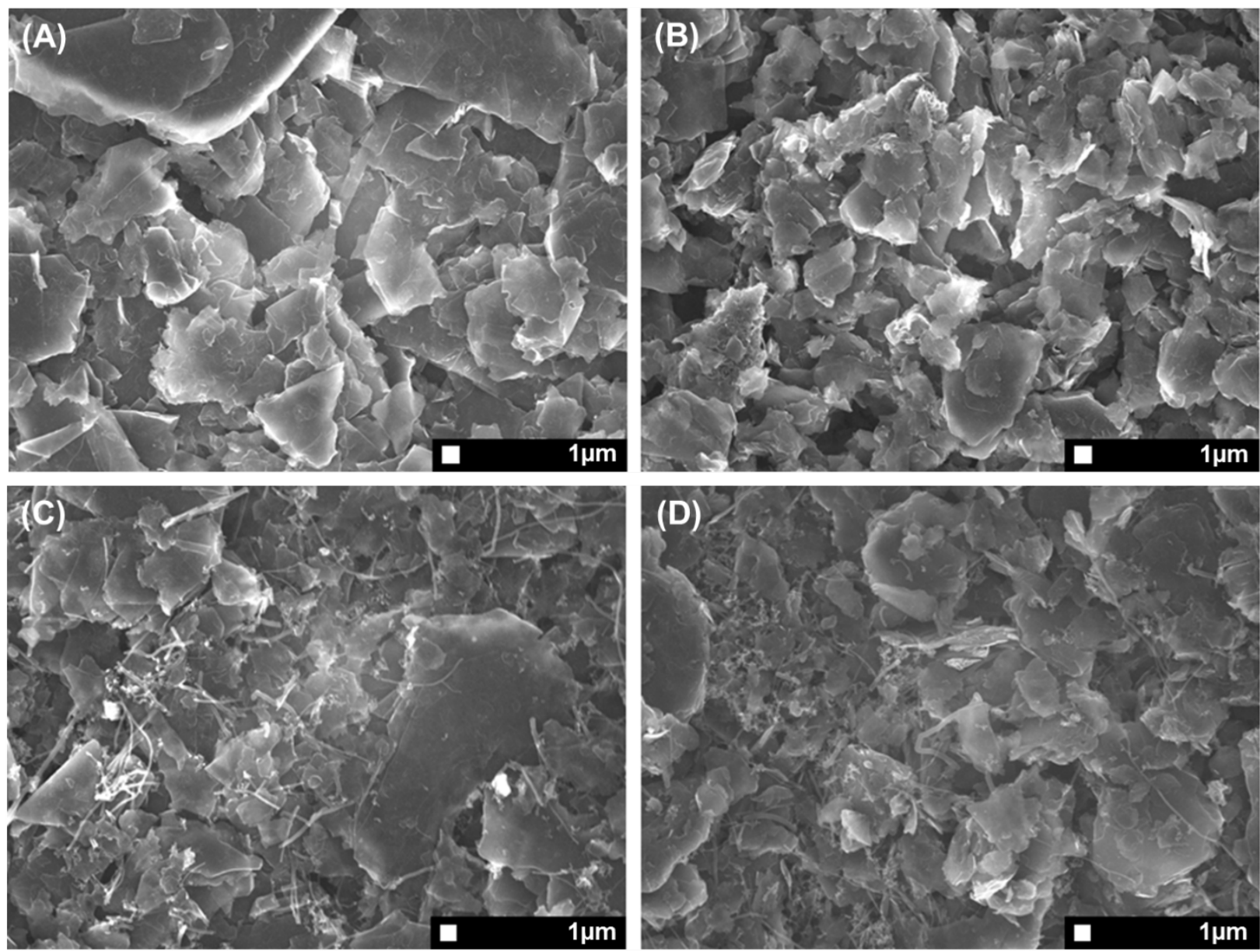

Figure 45 - SEM micrographs of different anode formulations (A) natural graphite (B) synthetic graphite (C) natural graphite with carbon additives (D) synthetic graphite with carbon additives.

The natural graphite selected for testing exhibited a higher initial capacitance than synthetic graphite. The initial capacitance achieved from the synthetic graphite with carbon additives was $64 \mathrm{mAh} / \mathrm{g}$, which is about half of the theoretical reversible capacitance of $\mathrm{LiCoO}_{2}$. The highest capacitance achieved from natural graphite in this testing was $112 \mathrm{mAh} / \mathrm{g}$, which is near double compared to the synthetic graphite and much closer to the theoretical reversible capacitance of $\mathrm{LiCoO}_{2}$ of $140 \mathrm{mAh} / \mathrm{g}$.

The lower capacity obtained with the synthetic graphite is due to the lower rhombohedral phase content, $8.2 \%$, with corresponding defect concentration. In this graphite, the lithium ions intercalation is predominantly on the surface layer, which prevents further insertion of ions into the graphite's interlayers [69]. This phenomenon is due to an accumulation of ions at the limited amount of defect sites during cell 
formation; therefore, a promoted electrolyte decomposition and an anchored growth of the SEI layer occurred [71], [79]. Thus, the natural graphite that contained a hexagonal phase content of $29.9 \%$, with corresponding defect concentration, was able to achieve a higher initial cell capacitance.

Unfortunately, the capacity fade during cycling of the natural graphite was an unacceptable 53\%, greater than the synthetic graphite at 14\% (Figure 46). Even with the higher capacity loss, the natural graphite was selected from this series of tests to be utilized in the rest of performance testing due to its high initial capacitance, with the next set of testing focused on minimizing this observed capacity loss.

\section{Capacity Fade}

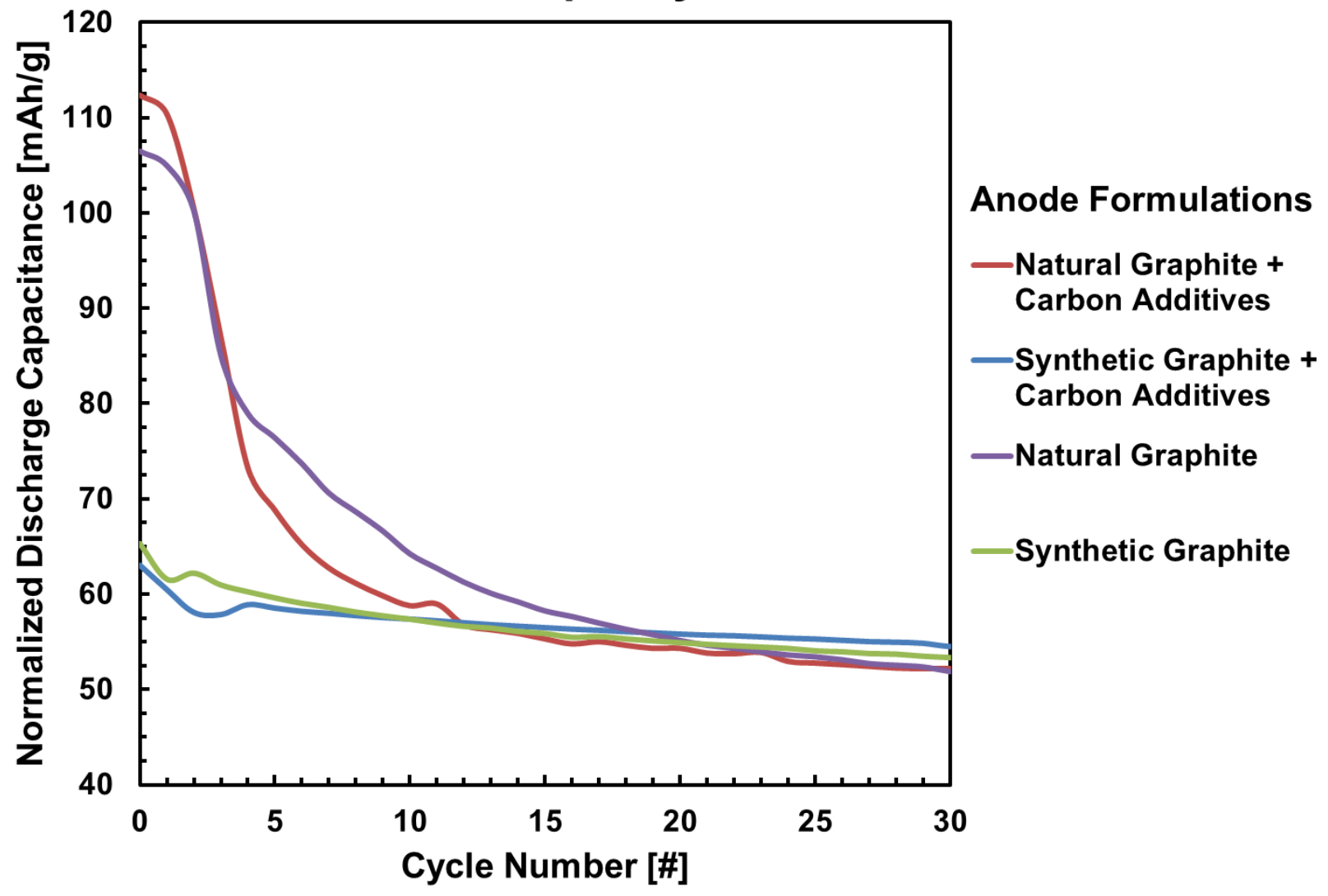

Figure 46 - Cycling data overlaying the capacity fade of different anode formulations.

The electrode formulations determined from this series of performance tests were a cathode of $\mathrm{LiCoO}_{2}$ (in-house synthesized) + carbon black (Timcal, Super C45) + carbon nanofiber (Pylograph, PR-19-XT-LHT) + PVDF (Alfa Aesar, 44080) at (80/5/5/10 
vol\%), and an anode of natural graphite (Alfa Aesar, t2N5) + carbon black (Timcal, Super C45) + carbon nanofiber (Pylograph, PR-19-XT-LHT) + PVDF (Alfa Aesar, $44080)$ at $(82 / 4 / 4 / 10$ vol\%). This formulation was utilized to fabricate electrodes for the rest of the battery tests.

\subsubsection{Solid Electrolyte Interface Formation}

The formation of the solid electrolyte interface is largely dependent upon the graphitic materials in the anode and the electrolyte being utilized in the battery. This series of performance tests were conducted to determine the best protocol for forming a highly functional SEI layer with the revolutionary combination of materials fabricated in this research. Due to the various theories of SEI formation found throughout literature, the incorporation of nanoparticles throughout the anode could completely change the required formation protocol to correctly form a thick dense SEI layer. An insufficiently thick SEI layer will cause an immediate increase in the irreversible capacity loss by allowing the rapid exfoliation of the graphite structure combined with electrolyte decomposition with the graphite. Furthermore, the addition of carbon nanoparticles within the fabricated anode that increase the cells capacitance could also cause a higher irreversible capacity loss. The SEI layer is known to continue to increase with a decrease of particle size due to the fast breakdown of the weaker small particles, leading to a growth of the SEI layer over more particles. This performance test (Figure 47) was required to ensure that the in-house fabricated cells would fully form the SEI layer, allowing them to perform at their maximum potential. 


\section{Solid Electrolyte Interface Formation Cell Test}

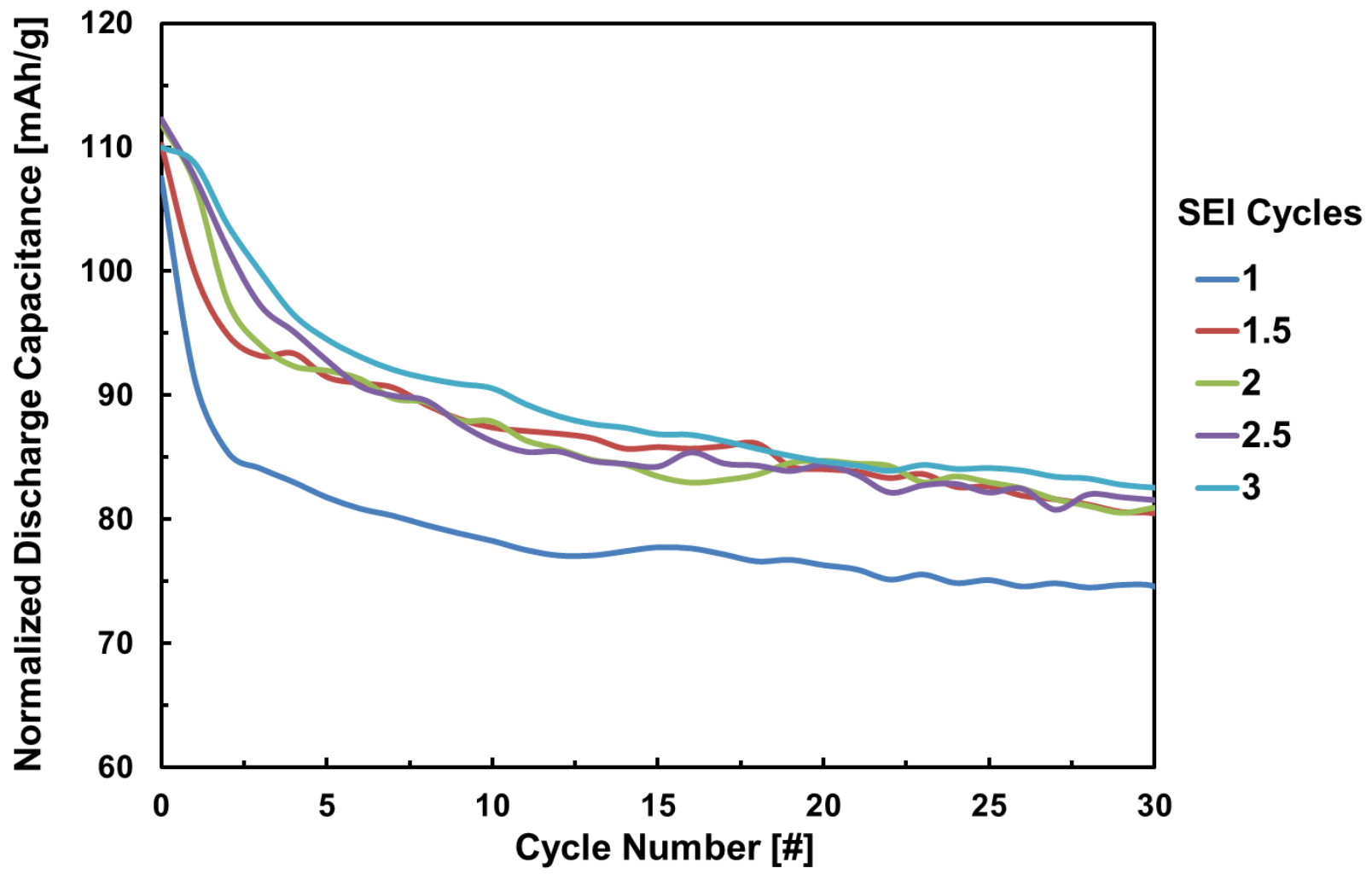

Figure 47 - Solid electrolyte interface cycling formation tests.

The protocol for this series of test cells was conducted at a slow rate determined by calculating the theoretical capacitance of each cell and cycling it at a C/25 rate. For this data a cycle includes both the charge and discharge portion to complete 1 cycle, whereas 0.5 means that the cell was subject to half or only the charge part of cycle. Each cell was then cycled at a consistent $\mathrm{C} / 5$ rate based on the actual capacitance for 30 cycles (Table 6$)$. 
Table 6 - Capacity loss [\%] for each SEl formation, along with the observed loss through 30 cycles.

\begin{tabular}{|r|r|r|}
\hline $\begin{array}{l}\text { SEI } \\
\text { Cycles }\end{array}$ & $\begin{array}{l}\text { SEI Formation } \\
\text { Capacity Loss [\%] }\end{array}$ & $\begin{array}{l}\text { Cycling Capacity } \\
\text { Loss [\%] }\end{array}$ \\
\hline 1 & 15 & 30.6 \\
\hline 1.5 & 13.8 & 26.9 \\
\hline 2 & 12.8 & 27.6 \\
\hline 2.5 & 9.27 & 27.3 \\
\hline 3 & 9.16 & 24.9 \\
\hline
\end{tabular}

Extrapolated from this series of tests is that due to the innovative combination of particles throughout the cell, the full formation of the SEl layer was not nearly completed during one formation cycle, which lead to a capacitance loss of $31 \%$ over 30 cycles. Actually, the stability of the cell continues to improve with each increased slow cycle resulting in the lowest irreversible loss of $25 \%$ after 30 cycles by conducting 3 full SEI cycles. However, the test cell conducted with a protocol of 1.5 cycles only experienced a capacity loss of $27 \%$ once cycling was completed. Therefore, due to the substantial amount of time required to complete just one cycle, experimentally between 25-30 hours per half cycle, a SEI formation protocol of 1.5 cycles was selected to be utilized throughout the rest of the battery testing.

\subsubsection{Off-the-Shelf Powder vs. In-House Synthesized Powder}

The last test conducted before continuing on to the next phase of the research was required to compare how the in-house synthesized cathode powder performed compared to an off-the-shelf purchased cathode powder. Even though a functional $\mathrm{LiCoO}_{2}$ cathode powder had been proven to be synthesized, a comparison to a readily available off-the-shelf powder was required to determine if the produced powder could perform at the same quality or better. These tests directly compared the capacity and fade due to cycling of both cathode powders. For this, a pure phase $\mathrm{LiCoO}_{2}$ cathode powder (Sigma-Aldrich, 442704) was obtained and compared to the in-house processed powder through microstructure characterizations of X-Ray diffraction and scanning 
electron microscopy (Section 3.5.2). Furthermore, the in-house cathode powder was subjected to a parallel evaluation at different charge and discharge levels to evaluate the capacitance fade seen at different rates (Figure 48).

All of the processing steps for fabricating the electrodes through manufacturing coin cells were performed in the same manner, regardless of the cathode powder utilized. The SEI formation cycle for these tests was 1.5 cycles, where the capacitance of each cell was determined and then programmed according to the charge and discharge rate being evaluated. Cycling rates of the in-house cathode ranged from $\mathrm{C} / 5$ to $1 \mathrm{C}$ for 30 cycles, where an expected decrease in capacitance should be observed as the rate is increased. The rate for comparing the off-the-shelf powder to the in-house synthesized powder was at $\mathrm{C} / 5$ for 30 cycles.

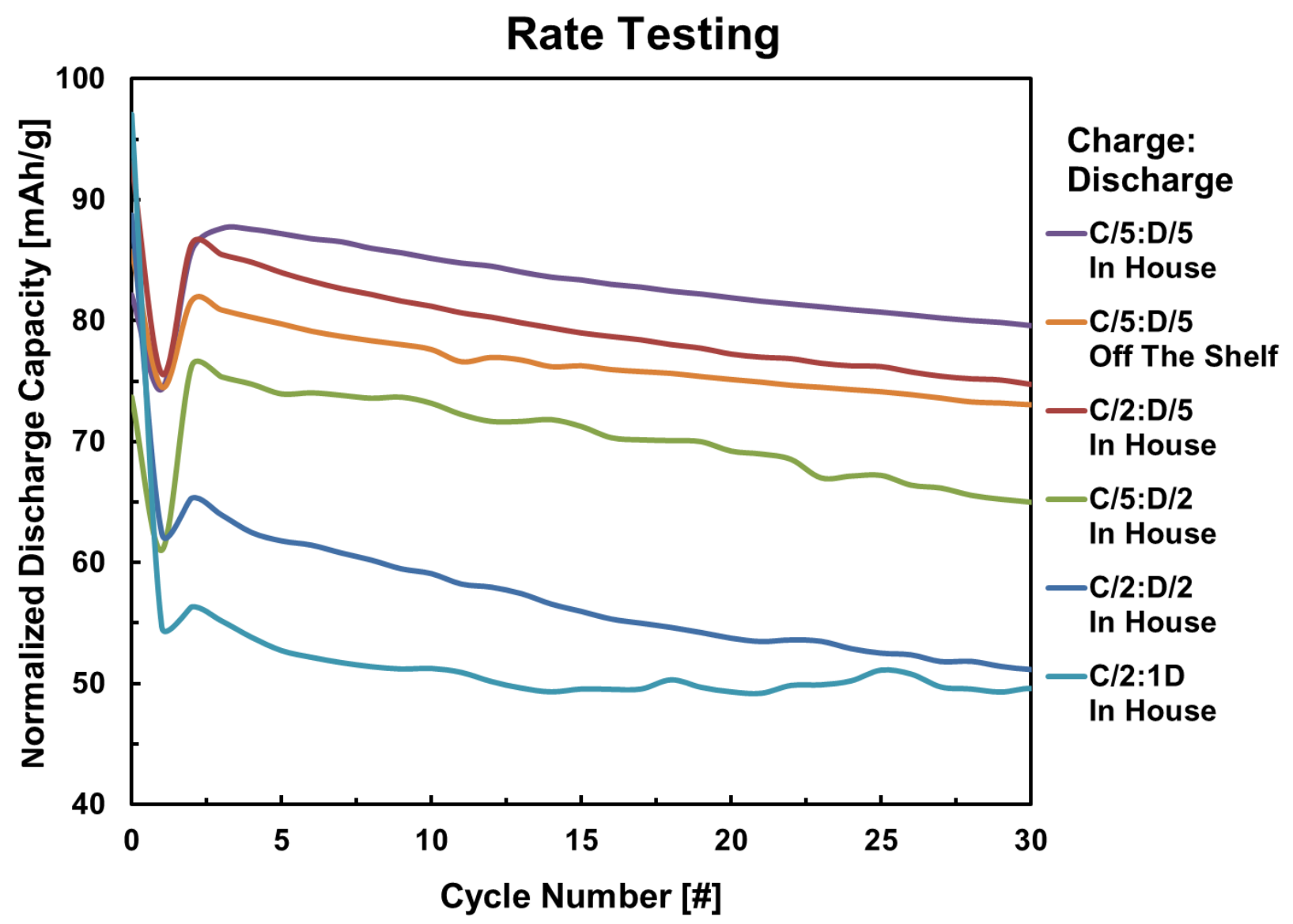

Figure 48 - Rate testing comparing in-house synthesized LiCoO2 powder to an off the shelf (Sigma Aldrich) $\mathrm{LiCoO}_{2}$ powder at different charge and discharge rates. The key represents the charge (C) and discharge $(D)$ rate related to the capacitance of the cell. 
The in-house cathode cells that were cycled at higher rates in parallel experienced a larger capacitance fade as expected. As the discharge and charge rates were increased, the initial capacity loss over the first cycle, after SEl formation, increased significantly. However, from these results it was also determined that retaining a slower charge rate aided in lowering the capacitance fade as the discharge rate was increased. A cell that was cycled at a $\mathrm{C} / 2$ rate experienced almost a $40 \%$ fade in capacity, whereas a cell that was discharged at $C / 2$ but charged at $C / 5$ only experienced a $20 \%$ fade in capacity over 30 cycles. In the end, this test determined the protocol that would be used for future testing.

In regards to comparing the in-house synthesized cathode powder to an off-theshelf cathode powder, the in-house synthesized powder had superior performance when cycling at the $\mathrm{C} / 5$ rate. Over 30 cycles, the in-house cathode cell only experienced a mere $3.3 \%$ fade in capacity, while the off-the-shelf cell experienced a larger $14 \%$ fade in capacity over cycling. Furthermore, an $8 \%$ increase in the normalized capacitance of the in-house cathode was observed. This result proved that the $\mathrm{LiCoO}_{2}$ cathode powder being manufactured in-house yielded higher performance results than what could be purchased, which allowed it to be confidently used for the rest of the battery testing. 


\section{Chapter 4: Electrochemical Evaluation of Multifunctional Materials}

\subsection{Introduction}

This chapter is the evaluation of the first path to fabricate a composite multifunctional lithium ion battery (Figure 19). This architecture consists of a structure with carbon fibers and fiberglass, incorporated within fiber-reinforced composites that act as active components within the battery structure. This structure exemplifies the true intent of a multifunctional battery. However, a decrease in performance is expected when compared to a traditional lithium-ion cell, due to the replacement of the traditional electrochemically active materials with alternative materials that are designed for structural utility (and not electrochemical activity).

This section is the first step in accomplishing truly multifunctional architecture, which was conducted to maximize the electrochemical potential of a carbon fiber weave by modifying it through various pretreatments. Electrochemical testing of the modified carbon fiber weaves were conducted in a traditional button cell platform. The performance for the use of the modified carbon fibers as the anode was then compared to the performance of conventional lithium ion materials to see which of the pretreatments improved the carbon fiber's performance.

The largest survey of material properties found through electrochemical testing of fibrous fabrics has been conducted by the US Army Research Laboratory. Their research was comprised of electrochemical testing on readily available off-the-shelf carbon materials in novel lithium ion battery structures. Their results showed that PAN based carbon fiber materials resulted in the best overall electrochemical capacitance, as well as sustaining the widest breadth of applicability for use in structural or textile applications [55]. Other groups have investigated further by trying to improve the electrochemical properties of carbon fibers by pretreatments such as heat or chemical modifications; however, most of the tested carbon fiber materials were individually processed and are not available commercially, In addition, each processed material was subjected to a single type of pretreatment [56], [57]. Another method alter the 
composite materials discussed throughout literature was to modify the carbon fiber material by pretreating it then incorporating the fibers within a traditional anode (not as a continuous weave) and performing electrochemical testing to increase performance of the novel lithium-ion cell [58], [59]. The impetus for this research was based on the fact that the electrochemical potential as a standalone anode of many of these different pretreatments on commercially available materials are largely unknown. These ideas were manipulated and integrated for the purpose of making a truly composite multifunctional battery.

\subsection{Carbon Fiber Experimental Treatments}

This research consisted of ten different treatments performed to carbon fibers, which were observed throughout literature; with the desire to maximize the electrochemical potential of carbon fiber in a lithium battery system [56], [57], [58], [59], [80], [81], [82]. The fibers received from the manufacturer are considered "sized" due to an epoxy or polymeric coating on the fiber surface. This coating is applied to the fiber strands for improved handling characteristics during manufacturing [83]. Sizing treatments can affect physical properties of the fiber, such as roughness, porosity, and surface functional groups. These alterations can reduce adhesion strength between the fibers and epoxy matrix. The pretreatments are expected to "desize" the fibers by removing this coating, as well as modify the functional groups on the surface of the fibers. The effect of each treatment of the fibers was characterized in Section 4.3, while the effect on the capacity and the cyclic ability of carbon fibers as a direct anode in a lithium ion battery system was also evaluated in Section 4.4. The ultimate goal is to derive empirical correlations between each treatments modification to the carbon fiber and the resulting electrochemical performance.

There are three types of carbon fiber samples evaluated in this research (Table 7). The properties of every carbon fiber are dependent on the fabrication process and source materials. There are two basic carbon fiber types, polyacrylonitrile (PAN) and pitch, and these terms are based upon the precursor polymers utilized in the fabrication of these fibers. PAN based fibers have a moderate graphite content, high strength, and low density; whereas, pitch based fibers contain a high graphite content, high modulus, 
high cost, high thermal conductivity, and high electrical conductivity [55], [84], [85]. Traditionally, pitch based fibers have been chosen for use in battery systems due to a high degree of graphitic content; however, research has shown that the structure of PAN based fibers have the capability of a higher capacity [57], [81]. Furthermore, carbon fibers utilized in this work are from both commercial grade and aerospace grade, each with its own advantages for anode functionality. The commercial grade is initially made from a large tow and then separated to make smaller tows; this process causes the fibers to be frayed and rough. The aerospace grade is initially made into a smaller tow, resulting in a clean smoother surface [56].

Table 7 - Manufacturer provided carbon fiber properties.

\begin{tabular}{|c|c|c|c|}
\hline Brand & $\begin{array}{l}\text { The } \\
\text { Composites } \\
\text { Store (CST) }\end{array}$ & $\begin{array}{l}\text { Cytec } \\
\text { Thornel } \\
\end{array}$ & $\begin{array}{l}\text { Cytec } \\
\text { Thornel } \\
\end{array}$ \\
\hline Type & PAN & PAN & Pitch \\
\hline Grade & $\begin{array}{r}\mathrm{T}-300 \\
\text { Commercial }\end{array}$ & $\begin{array}{r}\mathrm{T}-300 \\
\text { Aerospace }\end{array}$ & $\begin{array}{r}\mathrm{P}-25 \\
\text { Aerospace }\end{array}$ \\
\hline Tow & $1 \mathrm{~K}$ & $3 \mathrm{~K}$ & $2 \mathrm{~K}$ \\
\hline Epoxy Sizing wt\% & 1 & 1 & 1 \\
\hline Filament Diameter [ $\mu \mathrm{m}]$ & 7 & 7 & 10 \\
\hline Density $\left[\mathrm{g} / \mathrm{cm}^{3}\right]$ & 1.76 & 1.76 & 1.92 \\
\hline Fiber Tensile Strength [MPa] & 3530 & 3750 & 1560 \\
\hline Fiber Tensile Modulus [GPa] & 230 & 231 & 159 \\
\hline Electrical Resistivity $[\mu \Omega \cdot m]$ & 17 & 18 & 12 \\
\hline
\end{tabular}

The treatments conducted on the fibers consisted of three atmospheric treatments while heating the fibers in a tube furnace and holding a temperature of $200^{\circ} \mathrm{C}$ and $400^{\circ} \mathrm{C}$ for 8 hours in each environment, respectively. The three atmospheres selected were air, argon, and forming gas $\left(95 \% \mathrm{~N}_{2} / 5 \% \mathrm{H}_{2}\right)$. The acid pretreatments selected were $1 \mathrm{M}$ Hydrochloric $(\mathrm{HCl}), 1 \mathrm{M}$ Nitric $\left(\mathrm{HNO}_{3}\right)$, and $1 \mathrm{M}$ Sulfuric $\left(\mathrm{H}_{2} \mathrm{SO}_{4}\right)$. The process for these were soaking the fiber in the acid for 8 hours then rinsing them with deionized water where they were then transferred into an inert atmosphere to dry. After treatment each modified material was then characterized and electrically evaluated in a lithium ion battery system. 


\subsection{Characterization of Carbon Fiber Treatments}

The micro-characterization techniques employed to investigate the different carbon fiber modification pretreatments consisted of X-Ray photoelectron spectroscopy (XPS) (Physical Electronics PHI 5000 VersaProbe) and scanning electron microscopy (SEM) (JEOL JEM-7600F). SEM was used to view physical alterations of the fiber microstructure due to the impact from each treatment. XPS further analyzed this impact by determining the elemental compositions of $\mathrm{C}, \mathrm{O}$, and $\mathrm{N}$ on the surface of each fiber along with the fluctuating chemical structures in the carbon $\mathrm{C}$ 1s spectra peak. The PAN based commercial grade T-300 1K (CST) carbon fiber was the only fiber to receive all of the different pretreatments with corresponding microstructural investigations (Figures 49-58). Results of each respective evaluation are discussed below.
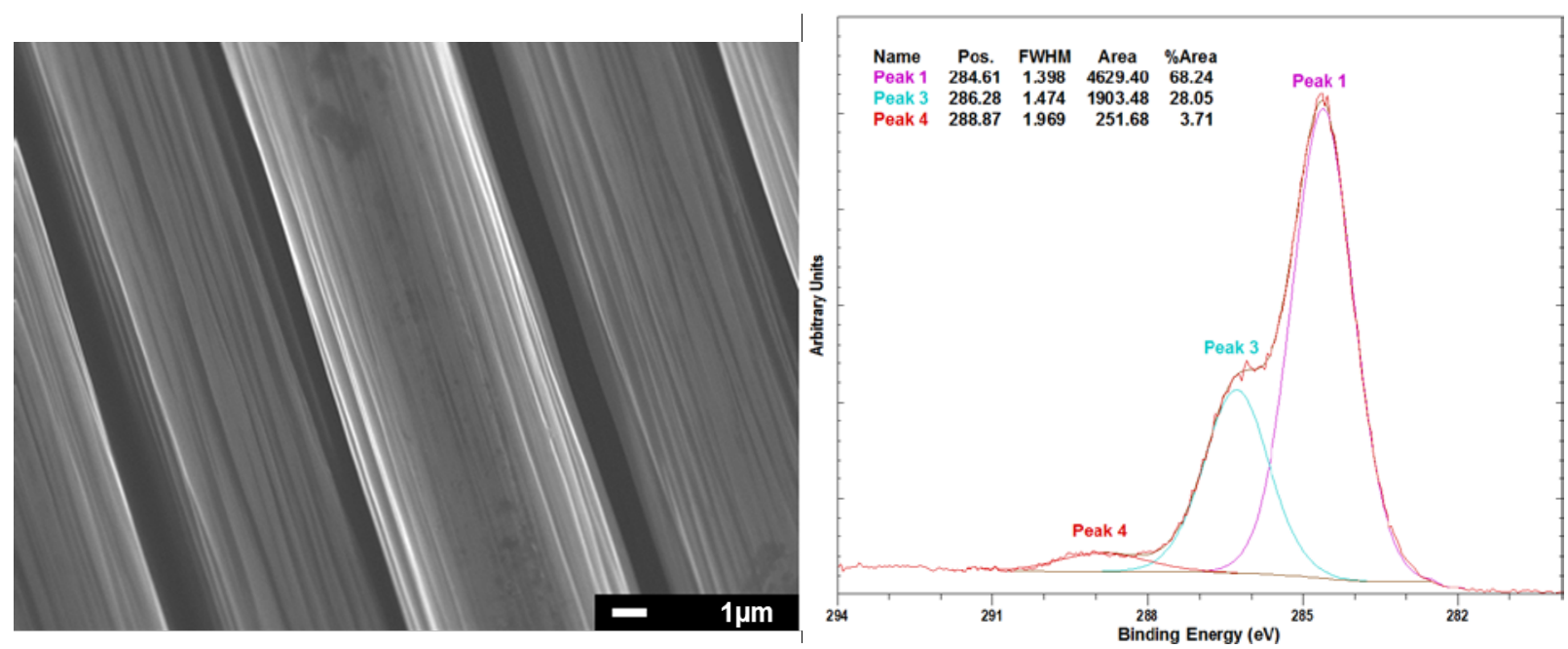

Figure 49 - SEM micrograph and corresponding XPS spectra deconvolution analysis of unmodified T-300 commercial grade fibers. 

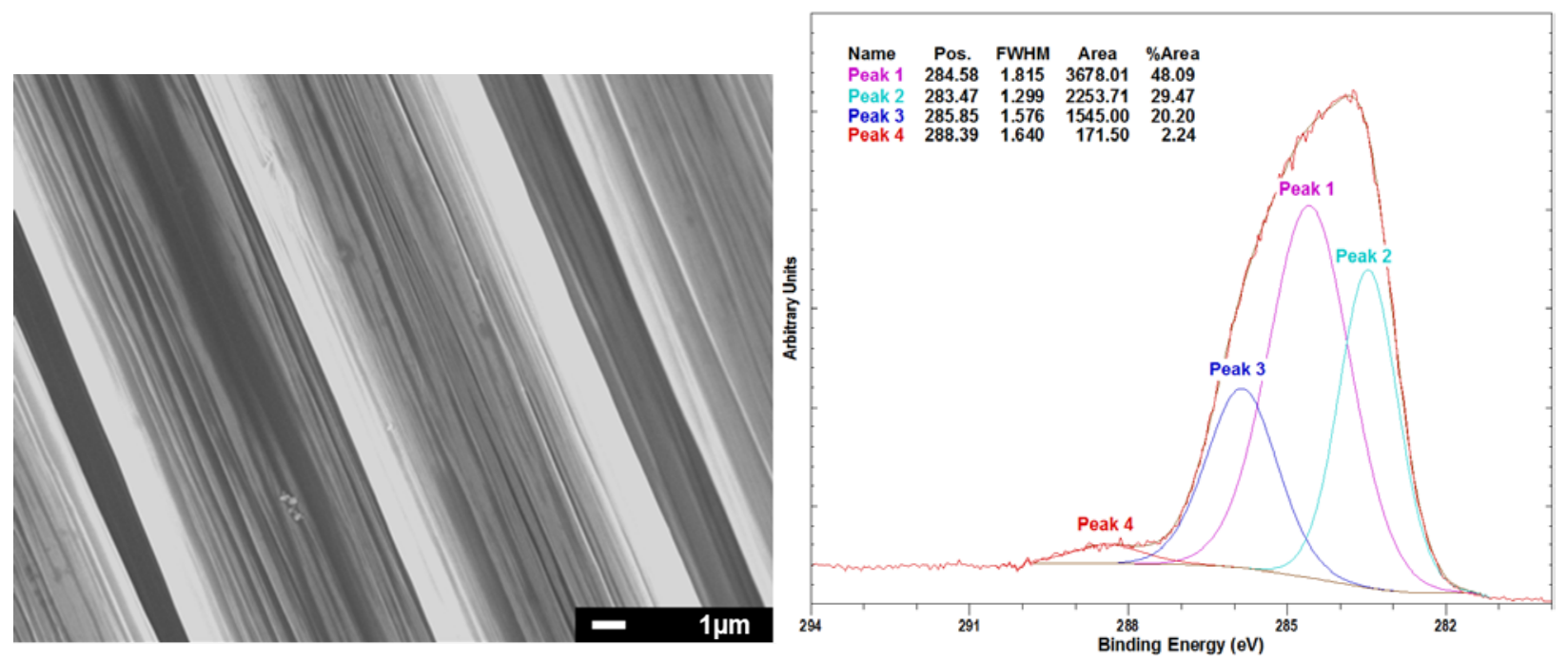

Figure 50 - SEM micrograph and corresponding XPS spectra deconvolution analysis of desized T$300 \mathrm{commercial}$ grade fibers by a heat treatment of $200^{\circ} \mathrm{C}$.
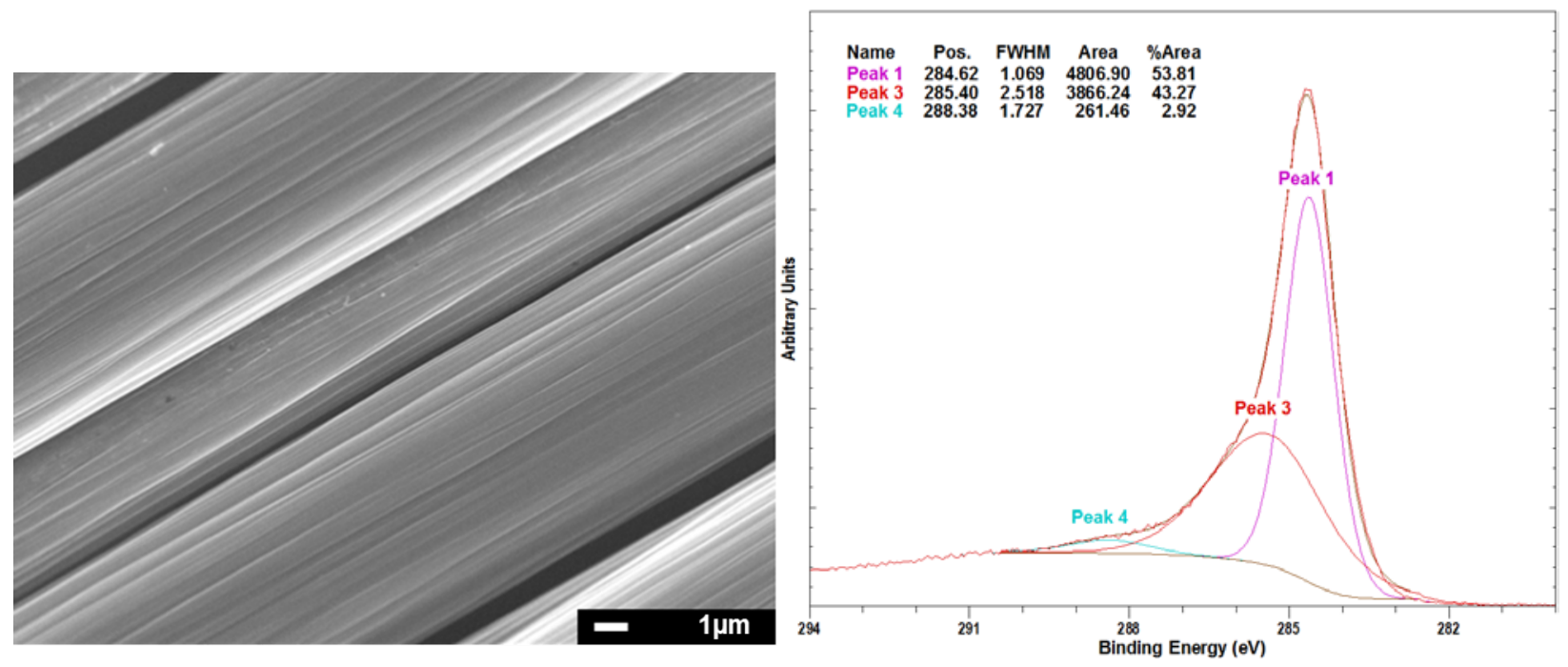

Figure 51 - SEM micrograph and corresponding XPS spectra deconvolution analysis of desized T$300 \mathrm{commercial}$ grade fibers by a heat treatment of $400^{\circ} \mathrm{C}$. 

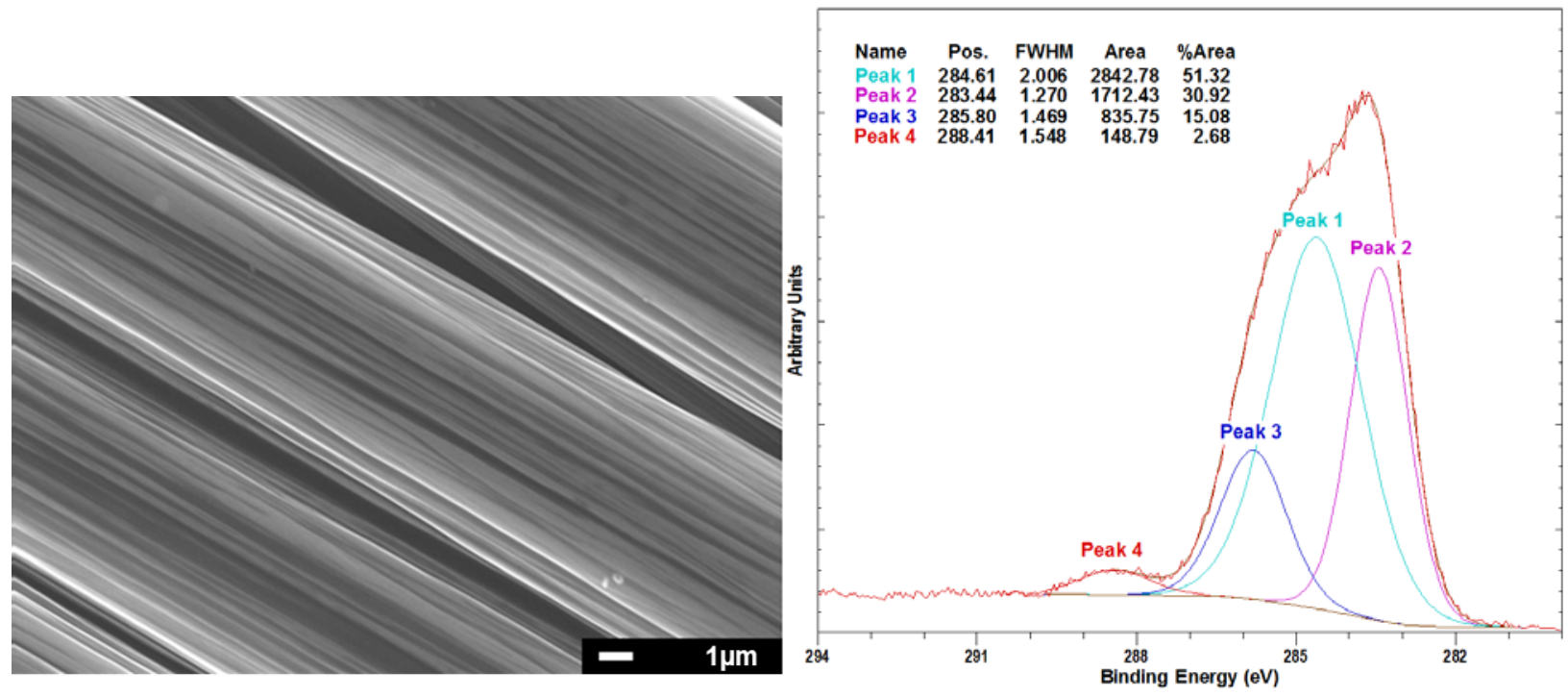

Figure 52 - SEM micrograph and corresponding XPS spectra deconvolution analysis of desized T300 commercial grade fibers by an inert atmospheric heat treatment at $200^{\circ} \mathrm{C}$.
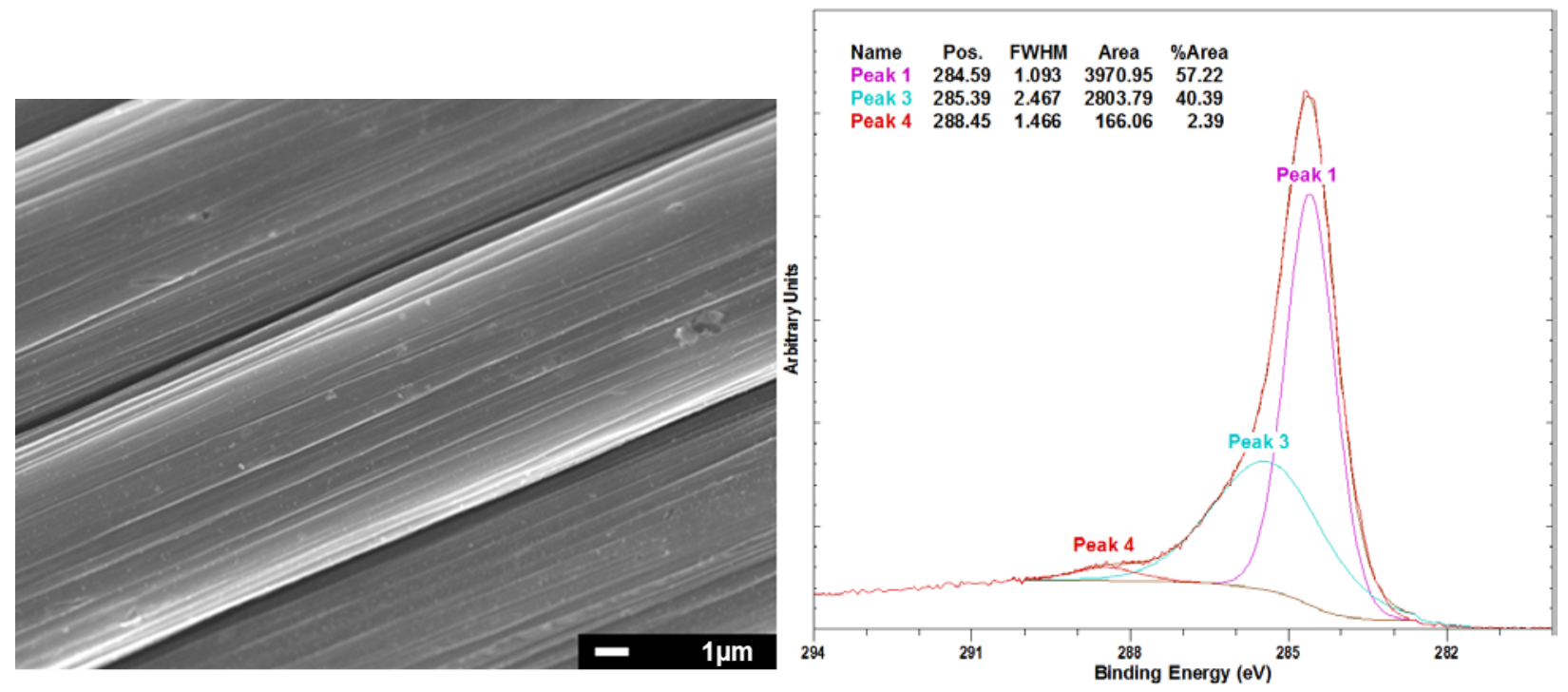

Figure 53 - SEM micrograph and corresponding XPS spectra deconvolution analysis of desized T300 commercial grade fibers by an inert atmospheric heat treatment at $400^{\circ} \mathrm{C}$. 

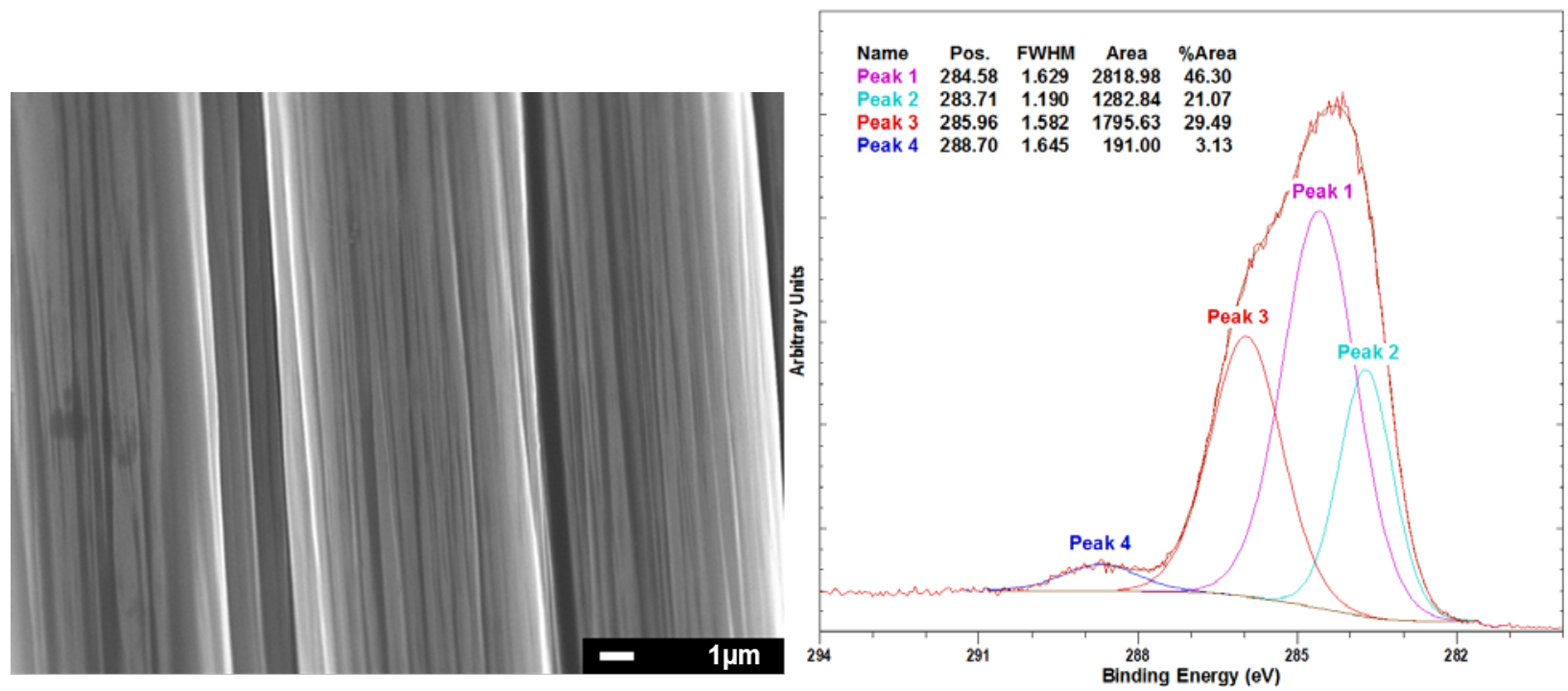

Figure 54 - SEM micrograph and corresponding XPS spectra deconvolution analysis of desized T300 commercial grade fibers by a reducing atmospheric heat treatment at $200^{\circ} \mathrm{C}$.
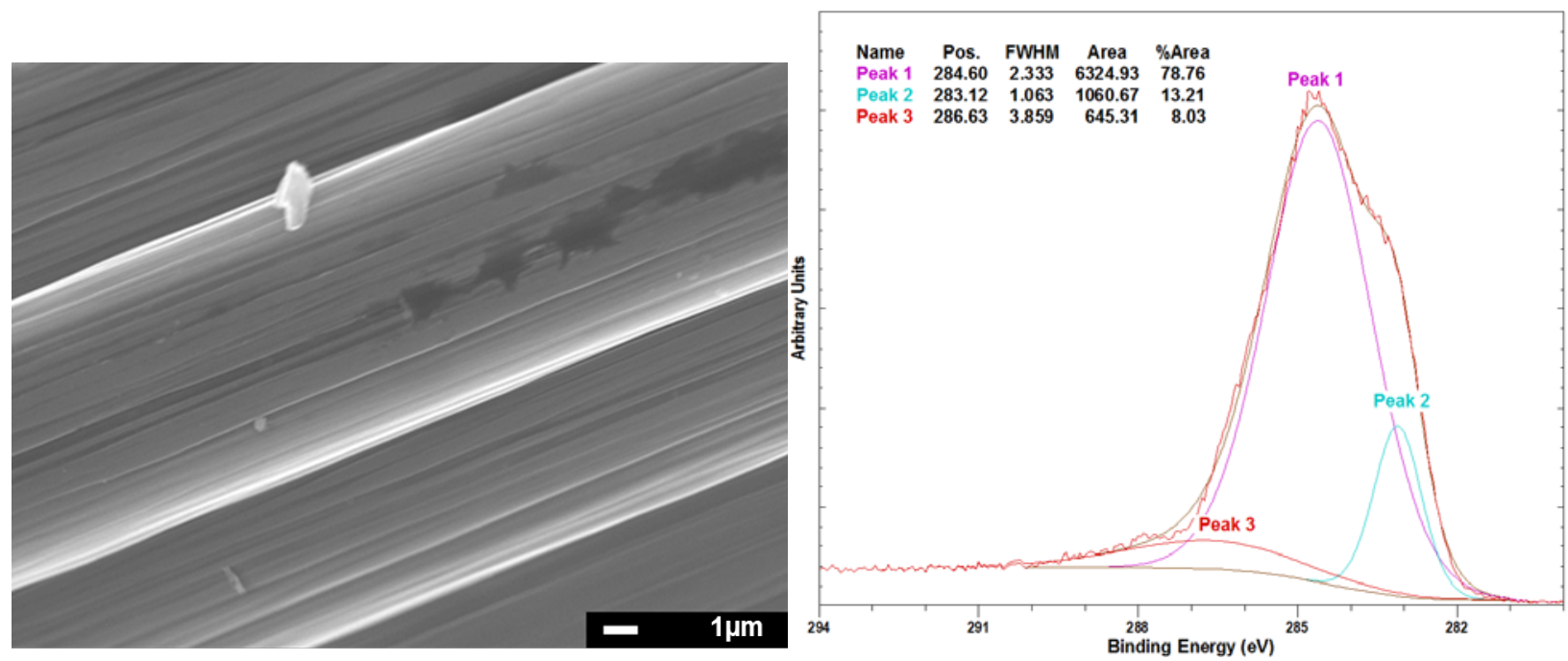

Figure 55 - SEM micrograph and corresponding XPS spectra deconvolution analysis of desized T300 commercial grade fibers by a reducing atmospheric heat treatment at $400^{\circ} \mathrm{C}$. 

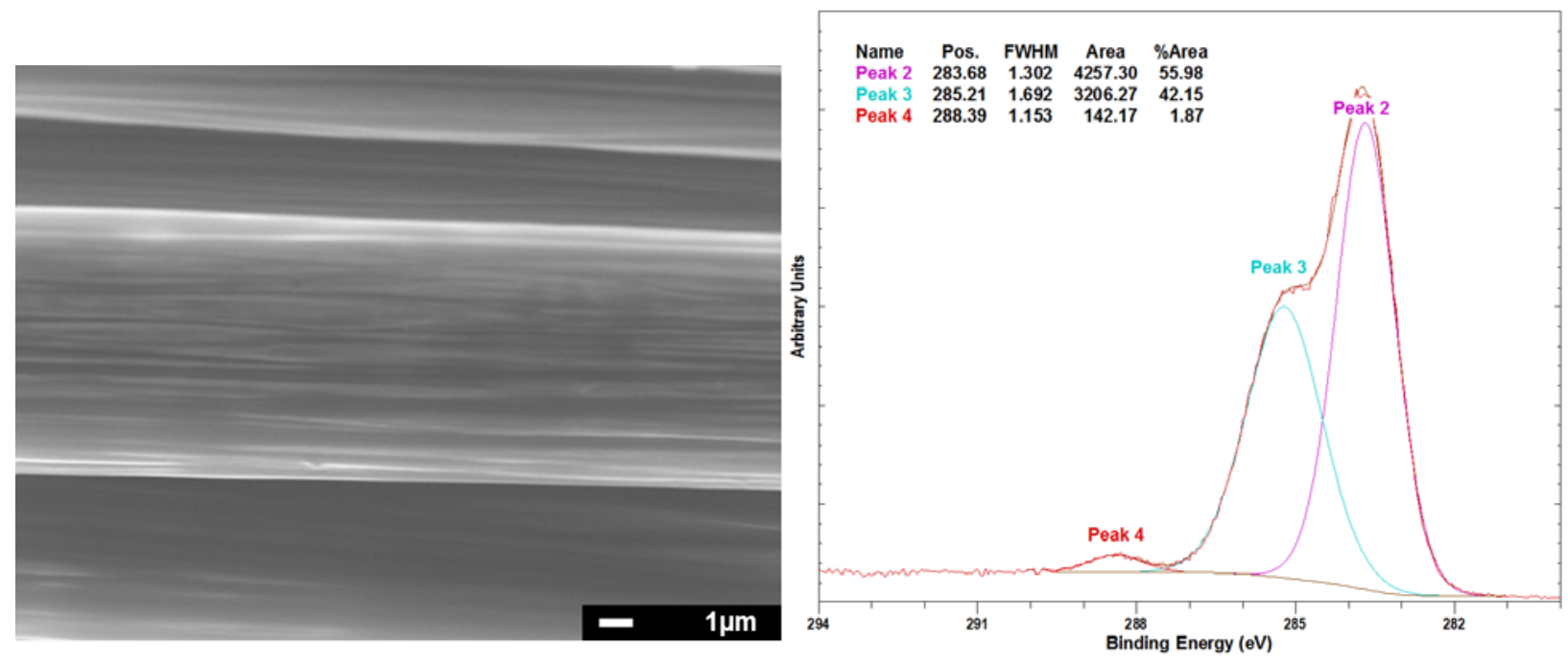

Figure 56 - SEM micrograph and corresponding XPS spectra deconvolution analysis of desized T300 commercial grade fibers by a $1 \mathrm{M}$ hydrochloric acid treatment.
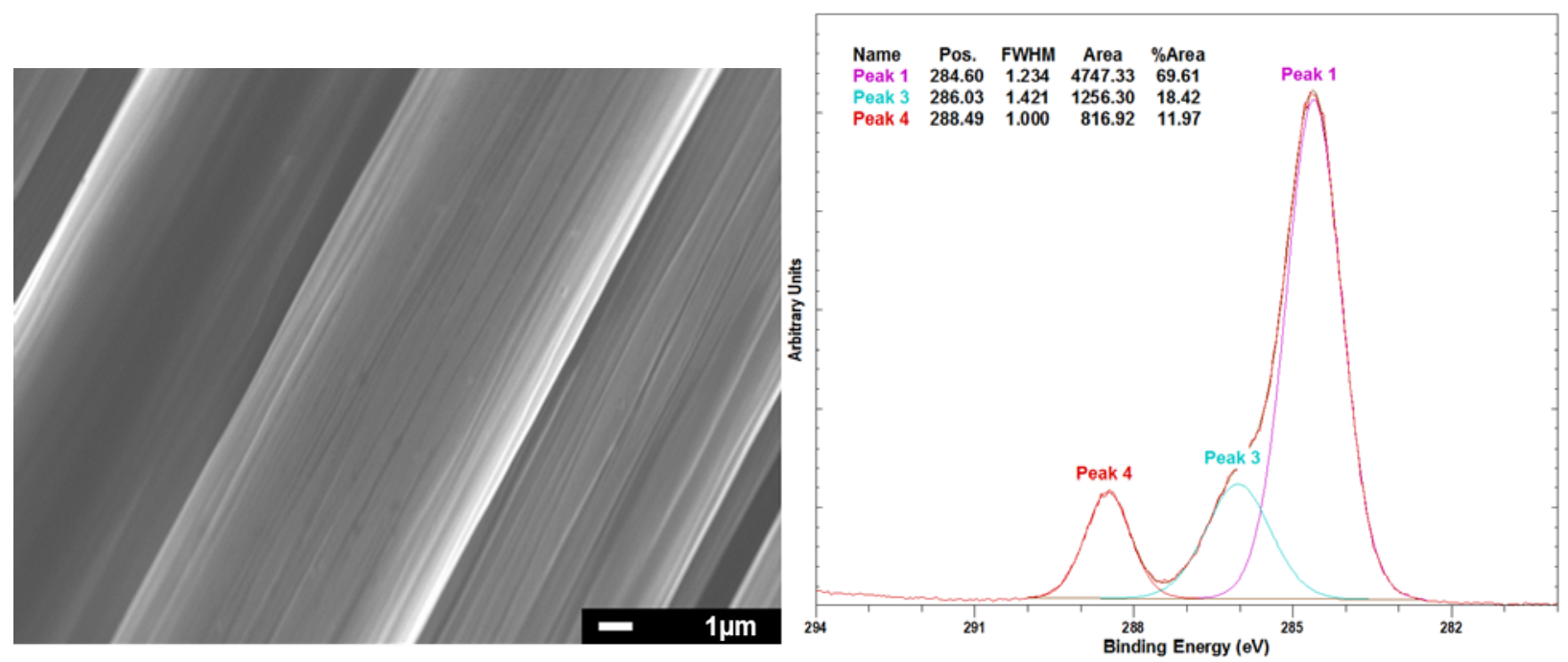

Figure 57 - SEM micrograph and corresponding XPS spectra deconvolution analysis of desized T300 commercial grade fibers by a $1 \mathrm{M}$ nitric acid treatment. 

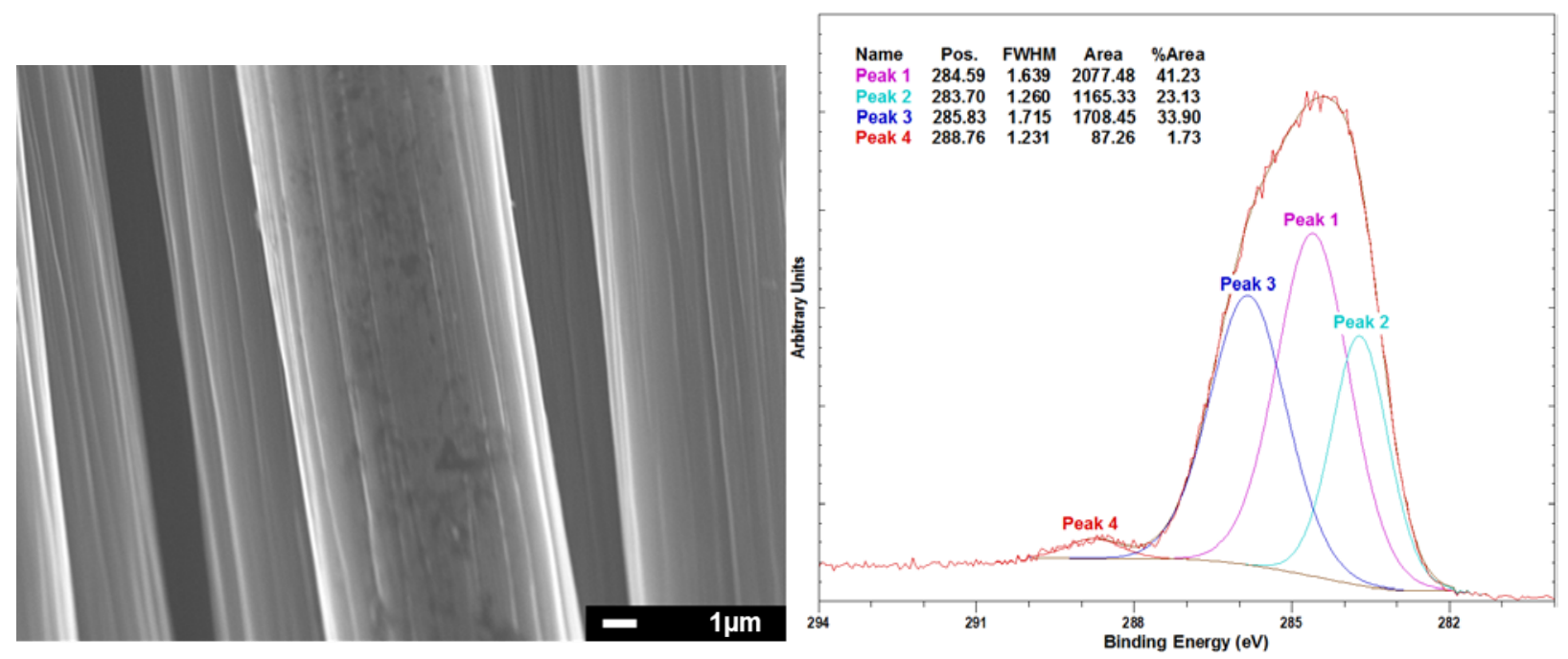

Figure 58 - SEM micrograph and corresponding XPS spectra deconvolution analysis of desized T300 commercial grade fibers by a $1 \mathrm{M}$ sulfuric acid treatment.

The scanning electron micrographs illustrate how each different pretreatment affected the actual fiber surface. There are distinct alterations to each pretreated sample. Each fiber that was subject to a heat treatment caused them to experience splitting along the fiber strand. Furthermore, the sulfuric acid and reducing atmospheric treatments appeared to create defects to the pores throughout the entire fiber surface. It was theorized that these alterations would increase the amount of lithium ions that could be inserted into the carbon fiber structure; therefore, resulting in an increase in initial cell capacitance [58], [59]

An in depth X-Ray photoelectron spectroscopy analysis, by CasaXPS processing software, of the different pretreatments performed to the carbon fiber samples show that there are significant differences in surface chemistry of the $C$ 1s spectra. A Shirley background was first applied to the spectra region prior to the peak analysis. The peak shape was conducted to investigate different surface functional groups by a deconvolution method of Gaussian with a 30\% Lorentzian contribution. The parameters selected for this analysis had been documented throughout literature discussing XPS studies on carbon fiber [86], [87], [88].

Throughout publications, there are small observed ranges of deconvoluted binding energies that refer to the different observed carbon based surface bond 
functional groups. These general peak binding energy groups were associated with their respective atomic concentration \% (Table 8).

Table 8 - XPS deconvolution to C 1s peak analysis of different pretreatment altered surface functional groups on carbon fiber.

\begin{tabular}{|c|c|c|c|c|c|c|c|c|}
\hline & \multicolumn{2}{|r|}{ Peak 2} & \multicolumn{2}{|c|}{ Peak 1} & \multicolumn{2}{|c|}{ Peak 3} & \multicolumn{2}{|r|}{ Peak 4} \\
\hline & $\begin{array}{l}\text { Binding } \\
\text { Energy } \\
{[\mathrm{eV}]}\end{array}$ & $\begin{array}{l}\text { Atomic } \\
\text { Concentration } \\
{[\%]}\end{array}$ & $\begin{array}{l}\text { Binding } \\
\text { Energy } \\
{[\mathrm{eV}]}\end{array}$ & $\begin{array}{l}\text { Atomic } \\
\text { Concentration } \\
{[\%]}\end{array}$ & \begin{tabular}{|l|} 
Binding \\
Energy \\
{$[\mathrm{eV}]$}
\end{tabular} & \begin{tabular}{|l|} 
Atomic \\
Concentration \\
{$[\%]$}
\end{tabular} & $\begin{array}{l}\text { Binding } \\
\text { Energy } \\
{[\mathrm{eV}]}\end{array}$ & $\begin{array}{l}\text { Atomic } \\
\text { Concentration } \\
{[\%]}\end{array}$ \\
\hline Untreated & - & \begin{tabular}{|c|}
- \\
\end{tabular} & 284.61 & $\begin{array}{r}68.24 \\
\end{array}$ & 286.28 & 28.05 & 288.87 & 3.71 \\
\hline Heat $200^{\circ} \mathrm{C}$ & 283.47 & 29.47 & 284.58 & 48.09 & 285.85 & 20.20 & 288.39 & 2.24 \\
\hline Heat $400^{\circ} \mathrm{C}$ & - & - & 284.62 & 53.81 & 285.38 & 43.27 & 288.37 & 2.92 \\
\hline Inert $200^{\circ} \mathrm{C}$ & 283.44 & 30.92 & 284.61 & 51.32 & 285.80 & 15.08 & 288.41 & 2.68 \\
\hline Inert $400^{\circ} \mathrm{C}$ & - & - & 284.59 & 57.22 & 285.36 & 40.39 & 288.42 & 2.39 \\
\hline Reducing $200^{\circ} \mathrm{C}$ & 283.71 & 21.07 & 284.58 & 46.30 & 285.96 & 29.49 & 288.70 & 3.13 \\
\hline Reducing $400^{\circ} \mathrm{C}$ & 283.12 & 13.21 & 284.60 & 78.76 & 286.63 & 8.03 & - & - \\
\hline Hydrochloric & 283.68 & 55.98 & - & - & 285.21 & 42.15 & 288.39 & 1.87 \\
\hline Nitirc & - & - & 284.60 & 69.61 & 286.03 & 18.42 & 288.49 & 11.97 \\
\hline Sulfuric & 283.70 & 23.13 & 284.59 & 41.23 & 285.83 & 33.90 & 288.76 & 1.73 \\
\hline
\end{tabular}

Peak 1 is the $\mathrm{C}-\mathrm{C}$ and $\mathrm{C}=\mathrm{C}$ bonding indicative of graphitic carbon. The Peak 2 energy shift refers to a carbidic group $\mathrm{C}-\mathrm{H}$ bonding and is symbolic of a precursor to graphitic carbon. The Peak 3 energy shift refers to hydroxyl and pyrrole groups with various $\mathrm{C}-\mathrm{O}, \mathrm{C}-\mathrm{OH}, \mathrm{C}-\mathrm{Cl}, \mathrm{C}-\mathrm{N}$, and $\mathrm{C}-\mathrm{NH}$ type bonding, dependent on the specific pretreatment. Finally, the Peak 4 energy shift refers to a carbonyl group composed of $\mathrm{C}=\mathrm{O}$ content [58], [59], [86], [88], [89], [90], [91].

The $\mathrm{HCl}$ pretreatment appeared to severely affect the surface functional groups by eliminating purely graphitic carbon bonding on the surface and replacing it with $\mathrm{C}-\mathrm{H}$ and $\mathrm{C}-\mathrm{Cl}$ functional groups. The reducing atmospheric at $400^{\circ} \mathrm{C}$ treatment completely eliminated any carbonyl $\mathrm{C}=\mathrm{O}$ content and created the highest content of graphitic carbon bonding. Furthermore, several treatments created $\mathrm{C}-\mathrm{H}$ surface groups that were not evident in the untreated fibers. This analysis of surface functional groups is further correlated in regards to resulting battery electrochemical performance in Section 4.5.

\subsection{Carbon Fiber Electrochemical Performance Testing}

This particular approach first created a typical novel lithium ion battery before continuing a focus on replacing the anode. The novel battery components in the approach used for an electrochemical testing baseline were an in-house solid state 
synthesized $\mathrm{LiCoO}_{2}$ powder as the cathode due to its high specific capacity of 140 $\mathrm{mAh} / \mathrm{g}$ [11], [28] and the anode was a typical natural graphite powder (Alfa Aesar, t2N5) that has a theoretical specific capacity of $372 \mathrm{mAh} / \mathrm{g}$ [31]. The cathode and anode materials were prepared for use by adding a polymer binder material of polyvinylidene fluoride (PVDF) (Alfa Asear, 44080) and a hybrid mixture of carbon nanofiber (Pyrograf Products, PR-19-XT-LHT) and carbon black (courtesy of Timcal, Super C45). Respective slurries were prepared in a solvent of N-Methyl-2-Pyrrolidinone (NMP) via solid state synthesis and tape cast onto aluminum (cathode) and copper (anode) foils at a thickness of $1 \mathrm{~mm}$. The tapes were then dried under vacuum $15 \mathrm{inHg}$ at room temperature $\sim 22^{\circ} \mathrm{C}$ for 12 hours to slowly remove the solvent at first, heat was then applied at $100^{\circ} \mathrm{C}$ for 12 hours under the vacuum to finish removing the solvent and drying the casts. The resulting baseline compositions were $\mathrm{LiCoO}_{2}+$ carbon black + carbon nanofiber + PVDF (80/5/5/10 vol\%) for the cathode and graphite + carbon black + carbon nanofiber + PVDF (82/4/4/10 vol\%) for the anode. The remaining novel battery components, which consists of the same separator and electrolyte materials utilized for the baseline testing, were a Celgard 2325 polypropylene/polyethylene (PP/PE) tri-layer separator material (courtesy of Celgard) and an electrolyte solution of $1 \mathrm{M}$ lithium hexafluorophosphate $\left(\mathrm{LiPF}_{6}\right)$ (Strem Chemicals, 03-0325) in ethylene carbonate (EC) (Alfa Aesar, A15735) + diethyl carbonate (DEC) (Alfa Aesar, A12477) (3:7 vol\%).

All of the electrochemical tests were conducted with CR2032 coin cells on MTI's 8 Channel Battery Analyzer. The protocol that each cell underwent was a slow formation charge at a rate of $\mathrm{C} / 40$ for 1.5 cycles to fully obtain a fluent solid electrolyte interface over the rough anode material. The formation of each cell was succeeded by cycling at a constant current/constant voltage (CC/CV) rate of $\mathrm{C} / 5$ to $\mathrm{C} / 20$ to $4.2 \mathrm{~V}$ followed by a CC discharge rate of $\mathrm{C} / 5$ to $3.0 \mathrm{~V}$ for 30 cycles each.

Once a baseline that was comparable to a typical lithium ion battery was achieved, experimentation with substitutions of the different anode composite materials into the battery for performance evaluations was conducted. The difference from the novel cell architecture to the composite cell is the carbon fibers completely replaced the graphite anode and respective current collector. This was conducted to create the 
environment of a truly composite multifunctional cell where the copper current collector would not be required due to the electrochemical and structural properties of the carbon fibers. The first series of carbon fiber electrochemical performance tests consisted of the pretreated PAN based commercial grade T-300 (CST) fiber (Figure 59).

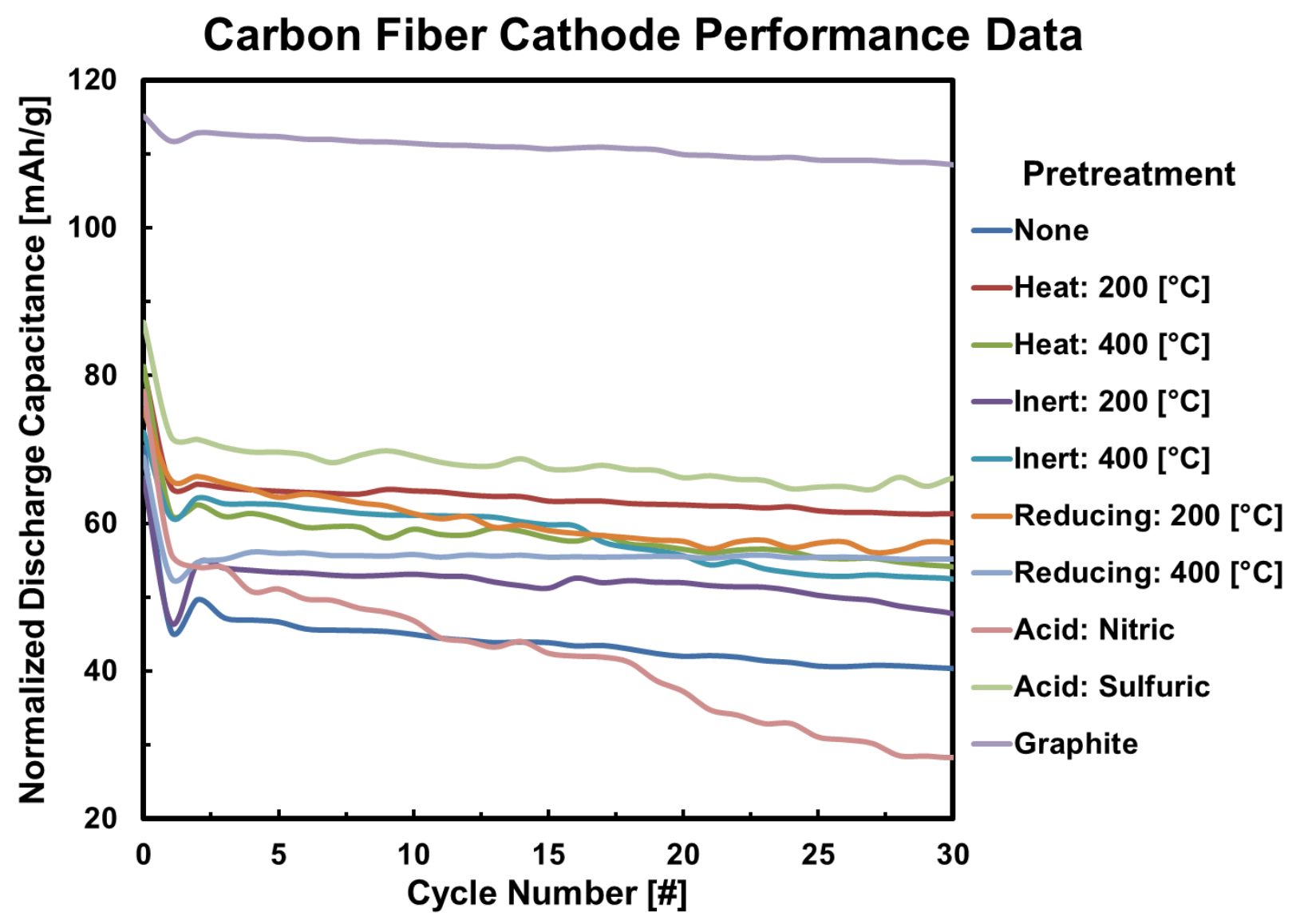

Figure 59 - Cathode based specific capacity electrochemical performance data of the different pretreatments conducted to the carbon fiber anode.

As expected, the novel lithium ion battery architecture baseline performance achieved a high reversible capacitance of $115 \mathrm{mAh} / \mathrm{g}$ followed by a low $5.7 \%$ capacity fade over 30 cycles, in regards to the cathode. Additionally, all of the performed pretreatments improved the electrochemical lithium ion battery potential of the untreated carbon fiber fabric. The exception to increased performance was the hydrochloric acid treatment, which never yielded a functional battery. The highest reversible capacity of $87 \mathrm{mAh} / \mathrm{g}$ was achieved by the sulfuric acid treatment, while the lowest capacity fade of $23 \%$, over 30 cycles, was achieved by the reducing atmospheric treatment at $400^{\circ} \mathrm{C}$. 
Furthermore, since the focus of this investigation was on the anode material, the same carbon fiber electrochemical performance data was analyzed in regards to the anode's specific capacity (Figure 60).

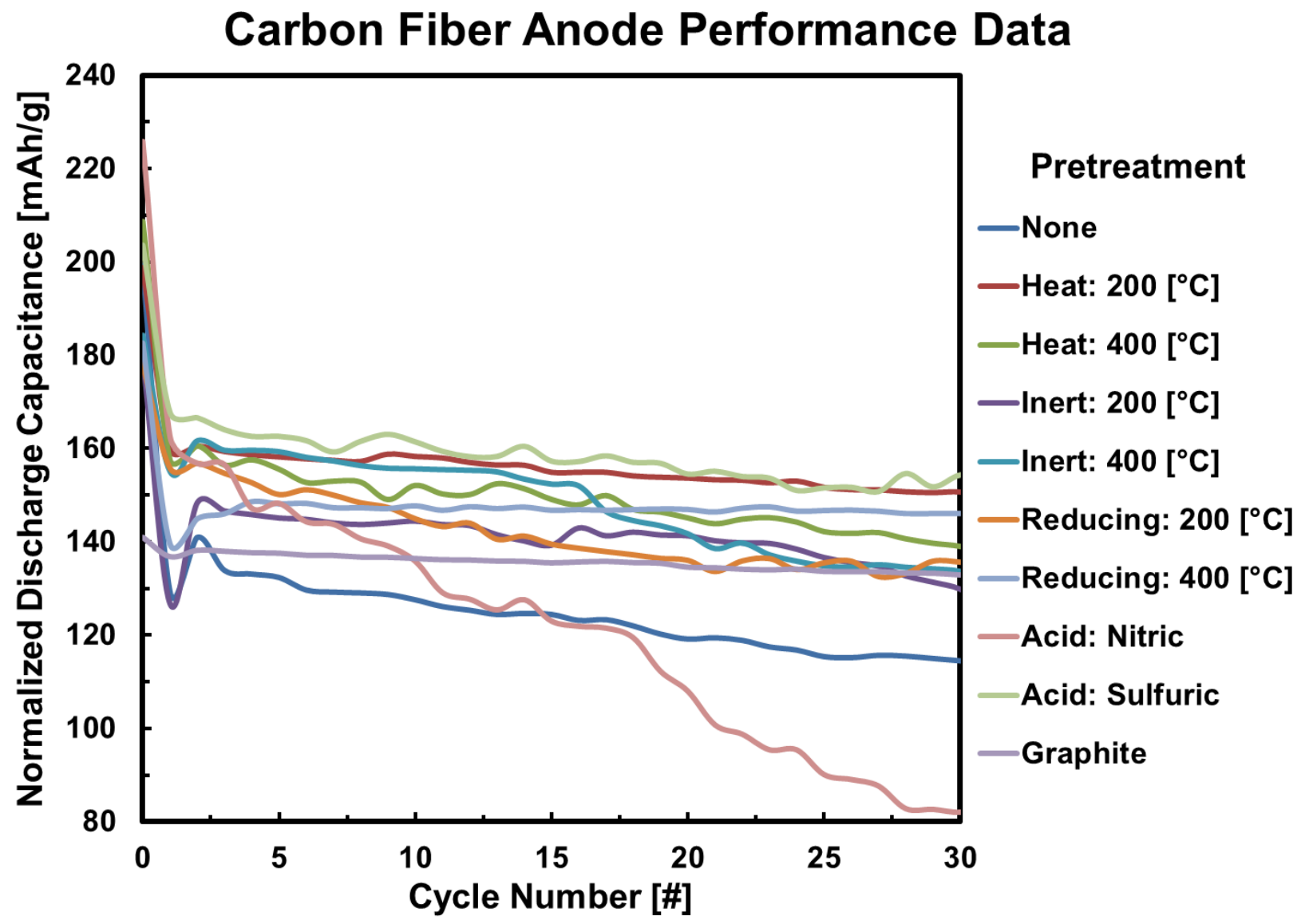

Figure 60 - Anode based specific capacity electrochemical performance data of the different pretreatments conducted to the carbon fiber anode.

The evaluation based on the specific capicity of the anode, derives that carbon fiber has a much higher capacity to mass ratio than a traditional graphite material. The highest specific reversible capacity achieved was $208.7 \mathrm{mAh} / \mathrm{g}$ from an atmospheric heat treatment of $400^{\circ} \mathrm{C}$. While, the graphite based anode demonstrated a mere 141 $\mathrm{mAh} / \mathrm{g}$ specific capacity. Furthermore, the graphite based anode did not take into account the current collecter required for fabrication mechanical stability. Even though the carbon fiber anode performed at a lower effiency than the traditional graphite anode, potential weight reductions achieved could compensate for the lower performance. 
It was projected that each of the previous pretreatments would similarly affect the structure of other carbon fiber types. Therefore, the electrochemical performance analysis should correspondingly change for each different treatment, regardless of precursor materials. The next series of carbon fiber electrochemical performance investigations consisted of an untreated fiber, a heat pretreatment of $200^{\circ} \mathrm{C}$, and a sulfuric acid pretreatment (Figure 61). The fibers utilized in this analysis was a PAN based T-300 3K fiber (Cytec Thonel) and a Pitch based P-25 2K fiber (Cytec Thonel).

\section{Carbon Fiber Cathode Performance Data}

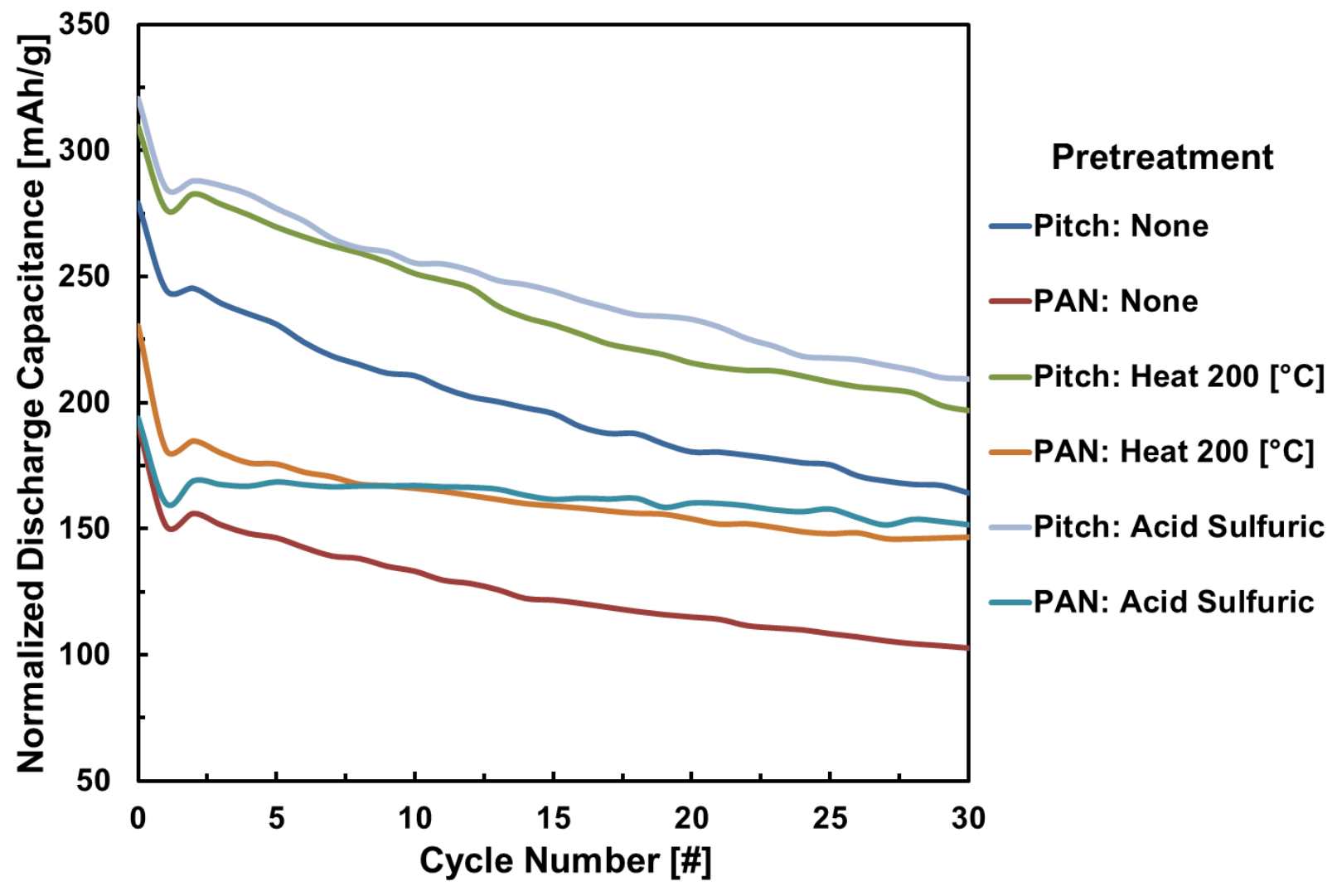

Figure 61 - Anode based specific capacity electrochemical performance data of PAN and Pitch based carbon fibers subject to the same pretreatments.

This evaluation concluded that, as predicted, the same pretreatment performed upon different types of carbon fibers would similarly affect their electrochemical potential in a lithium ion based battery. Both treatments increased the initial cell's capacitance, while the sulfuric acid treatment contributed to a lower capacity loss over cycling. Contradictory to literature, the pitch based fibers achieved higher capacities than the 
PAN based fibers; however, their capacity fade due to cycling was magnitudes higher [57], [81]. An in depth assessment of each fiber, respective pretreatment, and resulting electrochemical performance are exhibited for informative correlations that are difficult to evaluate based exclusively on the previous performance data (Table 9).

Table 9 - Electrochemical performance analysis of pretreated carbon fibers in respect to achieved capacity and resulting cyclic losses.

\begin{tabular}{|c|c|c|c|c|c|c|c|c|c|c|c|c|}
\hline \multirow[b]{3}{*}{ Supplier } & \multirow[b]{3}{*}{ Fiber } & \multirow[b]{3}{*}{ Tow } & \multirow[b]{3}{*}{ Treatment } & \multicolumn{6}{|c|}{ Carbon Fiber Capacity [mAh/g] } & \multirow{3}{*}{$\begin{array}{c}\text { Formation } \\
\text { Capacity } \\
\text { Loss [\%] }\end{array}$} & \multirow{3}{*}{$\begin{array}{c}\text { Cycling } \\
\text { Capacity } \\
\text { Loss 1st- } \\
\text { 30th [\%] }\end{array}$} & \multirow{3}{*}{$\begin{array}{l}\text { Cycling } \\
\text { Capacity } \\
\text { Loss 3rd- } \\
\text { 30th [\%] }\end{array}$} \\
\hline & & & & \multicolumn{3}{|c|}{ Lithiation Cycle } & \multicolumn{3}{|c|}{ Delithiation Cycle } & & & \\
\hline & & & & 1st & 3 rd & 30th & 1st & 3rd & 30th & & & \\
\hline Alfa Aesar & Graphite & - & - & 202.0 & 139.0 & 133.0 & 141.0 & 138.0 & 133.0 & 30.2 & 5.7 & 3.6 \\
\hline CTS & T-300 & $1 \mathrm{~K}$ & None & 399.8 & 138.7 & 115.2 & 199.3 & 133.9 & 114.5 & 50.2 & 42.5 & 14.5 \\
\hline CTS & $\mathrm{T}-300$ & $1 \mathrm{~K}$ & $\begin{array}{l}\text { Heat: } \\
200^{\circ} \mathrm{C}\end{array}$ & 363.1 & 161.1 & 151.6 & 198.8 & 159.3 & 150.7 & 45.2 & 24.2 & 5.4 \\
\hline CTS & $\mathrm{T}-300$ & $1 \mathrm{~K}$ & $\begin{array}{l}\text { Heat: } \\
400^{\circ} \mathrm{C} \\
\end{array}$ & 381.5 & 161.3 & 144.9 & 208.7 & 156.5 & 139.7 & 45.3 & 33.1 & 10.7 \\
\hline CTS & T-300 & $1 \mathrm{~K}$ & \begin{tabular}{|l} 
Inert: \\
$200^{\circ} \mathrm{C}$ \\
\end{tabular} & 365.5 & 150.0 & 131.5 & 179.8 & 146.6 & 129.8 & 50.8 & 27.8 & 11.5 \\
\hline CTS & $\mathrm{T}-300$ & $1 \mathrm{~K}$ & $\begin{array}{l}\text { Inert: } \\
400^{\circ} \mathrm{C}\end{array}$ & 325.7 & 161.7 & 136.3 & 184.3 & 159.6 & 133.7 & 43.4 & 27.5 & 16.2 \\
\hline CTS & $\mathrm{T}-300$ & $1 \mathrm{~K}$ & $\begin{array}{l}\text { Reducing: } \\
200^{\circ} \mathrm{C}\end{array}$ & 364.8 & 160.9 & 137.0 & 178.1 & 156.8 & 135.9 & 51.2 & 23.7 & 13.3 \\
\hline CTS & $\mathrm{T}-300$ & $1 \mathrm{~K}$ & $\begin{array}{l}\text { Reducing: } \\
400^{\circ} \mathrm{C}\end{array}$ & 346.8 & 149.4 & 146.1 & 189.6 & 148.6 & 146.1 & 45.3 & 23.0 & 1.7 \\
\hline CTS & T-300 & $1 \mathrm{~K}$ & $\begin{array}{l}\text { Acid: } 1 \mathrm{M} \\
\text { Nitric }\end{array}$ & 429.9 & 156.7 & 88.8 & 185.9 & 147.2 & 82.7 & 56.8 & 55.5 & 43.8 \\
\hline CTS & T-300 & $1 \mathrm{~K}$ & $\begin{array}{l}\text { Acid: 1M } \\
\text { Sulfuric }\end{array}$ & 381.3 & 167.0 & 154.7 & 203.6 & 166.6 & 154.3 & 46.6 & 24.2 & 7.4 \\
\hline Cytec Thornel & $\mathrm{T}-300$ & $3 K$ & None & 350.8 & 156.4 & 103.8 & 191.2 & 151.5 & 102.7 & 45.5 & 46.3 & 32.2 \\
\hline Cytec Thornel & $\mathrm{T}-300$ & $3 K$ & $\begin{array}{l}\text { Heat: } \\
200^{\circ} \mathrm{C}\end{array}$ & 382.9 & 185.5 & 146.5 & 230.4 & 180.0 & 146.3 & 39.8 & 36.5 & 18.7 \\
\hline Cytec Thornel & T-300 & $3 K$ & $\begin{array}{l}\text { Acid: } 1 \mathrm{M} \\
\text { Sulfuric }\end{array}$ & 346.7 & 169.2 & 154.5 & 193.8 & 167.5 & 151.6 & 44.1 & 21.8 & 9.5 \\
\hline Cytec Thornel & P25 & $2 \mathrm{~K}$ & None & 361.4 & 244.0 & 166.1 & 279.1 & 239.4 & 164.2 & 22.8 & 41.2 & 31.4 \\
\hline Cytec Thornel & P25 & $2 \mathrm{~K}$ & $\begin{array}{l}\text { Heat: } \\
200^{\circ} \mathrm{C}\end{array}$ & 393.1 & 282.6 & 197.1 & 309.5 & 278.6 & 196.8 & 21.3 & 36.4 & 29.4 \\
\hline Cytec Thornel & P25 & $2 \mathrm{~K}$ & $\begin{array}{l}\text { Acid: 1M } \\
\text { Sulfuric }\end{array}$ & 408.9 & 286.2 & 208.2 & 320.5 & 276.9 & 207.4 & 21.6 & 35.3 & 25.1 \\
\hline
\end{tabular}

Presented on Table 9 are the charge and discharge capacities of the different samples at their $1^{\text {st }}, 3^{\text {rd }}$, and $30^{\text {th }}$ cycles. The formation capacity loss is the initial irreversible capacity contributed to formation of the solid electrolyte interface SEI layer, along with the resulting capacity loss due to cycling. The analysis speculated that the SEI layer had not been fully formed in the first 1.5 cycles, and continued to grow throughout initial cycling. This phenomenon is likely due to the rough interfacial surface 
resulting from the fiber strands. This led to further investigation on the capacity loss from the $3^{\text {rd }}$ to the $30^{\text {th }}$ cycle of each cell. A much smaller capacity fade was evident for all of the fiber treatments, confirming that the SEI was still forming through the $3^{\text {rd }}$ cycle. Furthermore, a mere $1.7 \%$ fade in capacity was achieved by a reducing atmospheric treatment at $400^{\circ} \mathrm{C}$ from the $3^{\text {rd }}$ to $30^{\text {th }}$ cycle, prompting a huge $21.7 \%$ loss between the $1^{\text {st }}$ to $3^{\text {rd }}$ cycles.

\subsection{Discussion}

This goal of this section was to deliver an in depth evaluation of how different pretreatments performed to carbon fiber fabrics affected the electrochemical performance, as an anode, in lithium ion battery interactions. Nine different pretreatments were performed to one type of fiber and the resulting effects were characterized by SEM and XPS analysis.

The examination by SEM displayed distinct alterations of to the pretreated samples. These alterations were splitting of the fiber strands and defects created on the fibers surface, which allowed for more intercalation sites [58]. The effects of these sites on the capacity were not evident through the initial lithiation of ions into the carbon fiber; however, the reverse capacity loss achieved by most of the pretreatments was lower than the untreated sample.

Associations can be made from the XPS determined surface functional groups on the carbon fiber and resulting electrochemical performance. A dominant graphitic carbon phase must be present in the material. The hydrochloric pretreated fiber eliminated this functional group, resulting in failed electrochemical functioning in a battery. Furthermore, a higher concentration contributed from the carbonyl surface group resulted in higher capacitance losses. This is likely due to a large decomposition of the electrolyte with the $\mathrm{C}=\mathrm{O}$ molecules generating gases of $\mathrm{CO}$ and $\mathrm{CO}_{2}$ during the charging process [46], [48]. The stronger the bond of the oxygen, the harder it is to maintain the capacity throughout cycling [92]. This capacity loss is also due to partial exfoliation within the carbon fiber layers, resulting in extended growth of the SEI layer [48], [49]. However, treatments that lowered the surface oxygen content also lowered electrochemical interactions with the electrolyte solution. This inhibited the 
decomposition of electrolyte reactions on the carbon fiber surface, resulting in higher efficiencies throughout cycling [89].

This investigation determined that all of the pretreatments performed, except nitric and hydrochloric acids, positively affected the carbon fiber materials electrochemical properties. Substantial increases of the initial reverse capacitance, as well as a lower capacity loss throughout cycling were evident from the rest of the pretreatments. The exceptional pretreatments, in regards to electrochemical performance of carbon fiber in a lithium ion battery, were heat $200^{\circ} \mathrm{C}$, reducing $400^{\circ} \mathrm{C}$, and sulfuric acid. 


\section{Chapter 5: Mechanical Evaluation of Structural Cells}

\subsection{Introduction}

This chapter is a mechanical evaluation of the second path with the overall goal to fabricate a composite multifunctional lithium ion battery (Figure 20). The assessment was derived by modifying a typical multifunctional lithium ion pouch battery by creating perforations through the cell to increase bending strength and reduce delamination of internal components. This modified architecture allows for a highly formable battery, which when layered within a composite matrix, enhances the normal and shear strength. In this chapter, four-point beam flexure testing was used to characterize the relation between the perforation modifications and the structural strength, as well as the remaining capacity of each modified structural cell.

\subsection{Modifications to Classical Cell Architecture}

The aim of this task was to develop processing methods to create perforations (holes or "vias") through the cell, and characterize the effect of these vias on the functional battery area and flexural strength of the modified architectures.

The other institutions and companies currently working in the area of multifunctional structural batteries consist of a different approach by notably using offthe-shelf batteries in a structure instead of truly integrating the battery and structure together. One of their approaches focuses on removing internal polymer components of the functional structure and replacing them with commercial lithium ion cells sandwiched into structural panels. This eliminates the need for a secondary battery subsystem, which in turn reduces the volume and weight of the structure or craft. Testing showed that the alteration of internal structural components did not compromise the structure's required mechanical properties [10], [60]. Another proven example of combining the structure and battery is in unmanned air vehicles demonstrated in the Wasp micro air vehicle wing skin. The Wasp design approach added a structure function to already existent systems by stacking battery cells with a carbon fiber cloth layer between the separate cells, in combination with incorporating a carbon epoxy layer around them (Figure 18). This accomplished a lighter overall structure with enhanced mechanical strength leading to an overall success evident in longer flight times; however, the 
slightly customized cells designed to fit the Wasp cost thousands of dollars to purchase, and there are only a few manufacturers willing to fabricate customized cells [1], [60]. The approach of this research is to truly integrate the structure and battery into one combined and truly customizable multifunctional component.

\subsection{Composite Samples}

These samples were designed to lay the groundwork for fabricating object specific structural batteries. The design, fabrication, and testing of each configuration are discussed in this section.

\subsubsection{Design}

All of the mechanical samples tested were fabricated in a two-ply composite structure of carbon fiber fabric. The battery active area of these cells were calculated and utilized in the final capacity and current density calculations. The simulated battery samples did not contain the electrolyte solution for initial safety purposes, but further testing of this section include in-situ mechanical testing of fully-functional battery to determine effectiveness of this modified architecture under real world applications.

Twelve different architectures of test cells were manufactured in order to determine the optimal configuration of the perforations, which after deflection testing were related to the increased strength of the cell before delamination and reduced capacity of the cell due to the perforations. Each configuration was drawn in SolidWorks to illustrate the different derived architectures and corresponding labels (Figures 62-72). 


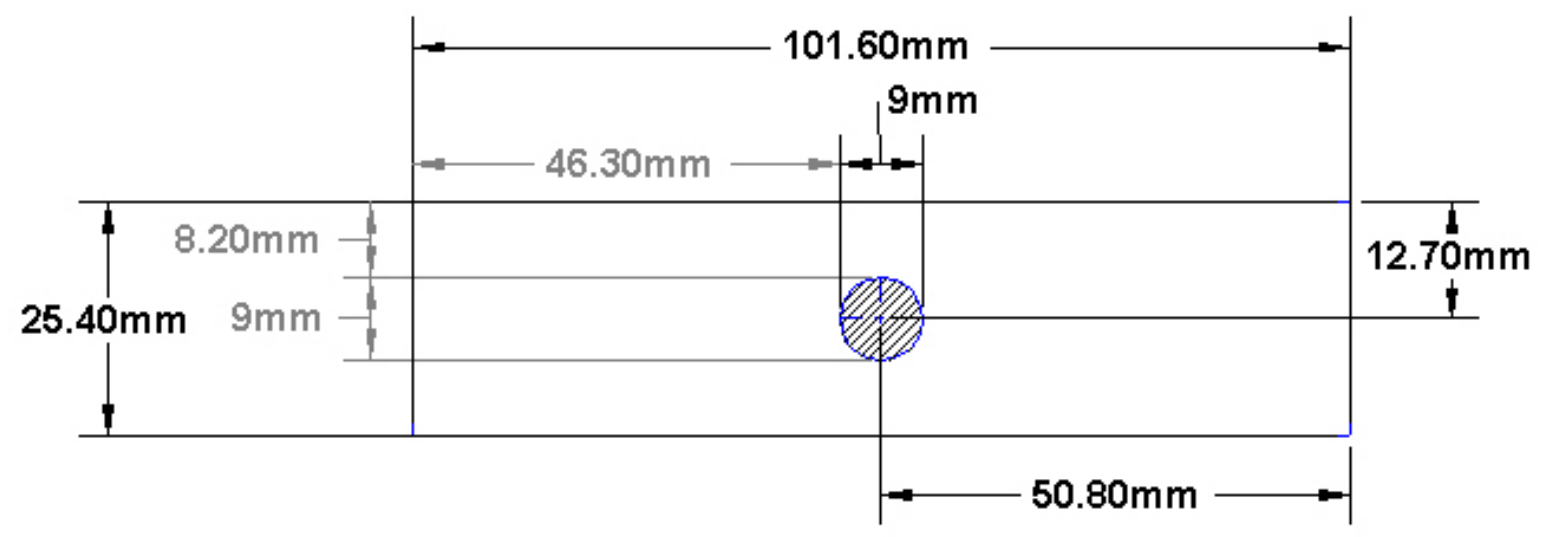

Figure 62 - Schematic of the one large via cell: 1.9.

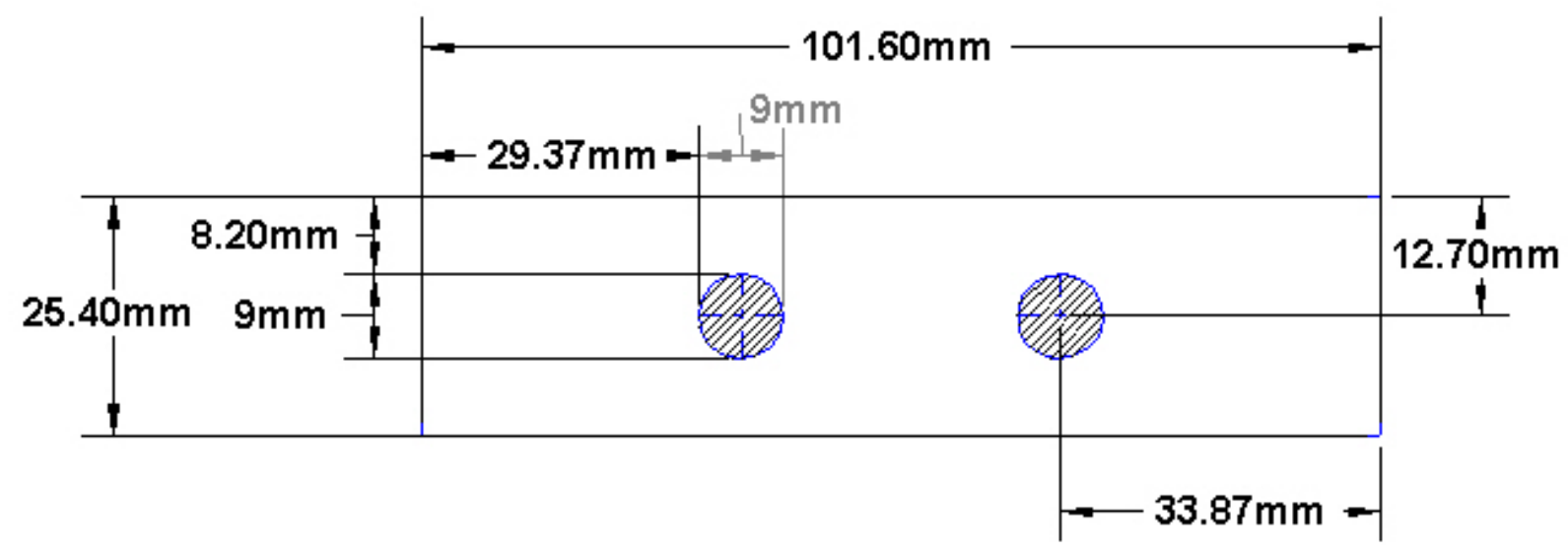

Figure 63 - Schematic of the two large via cell: 2.9.

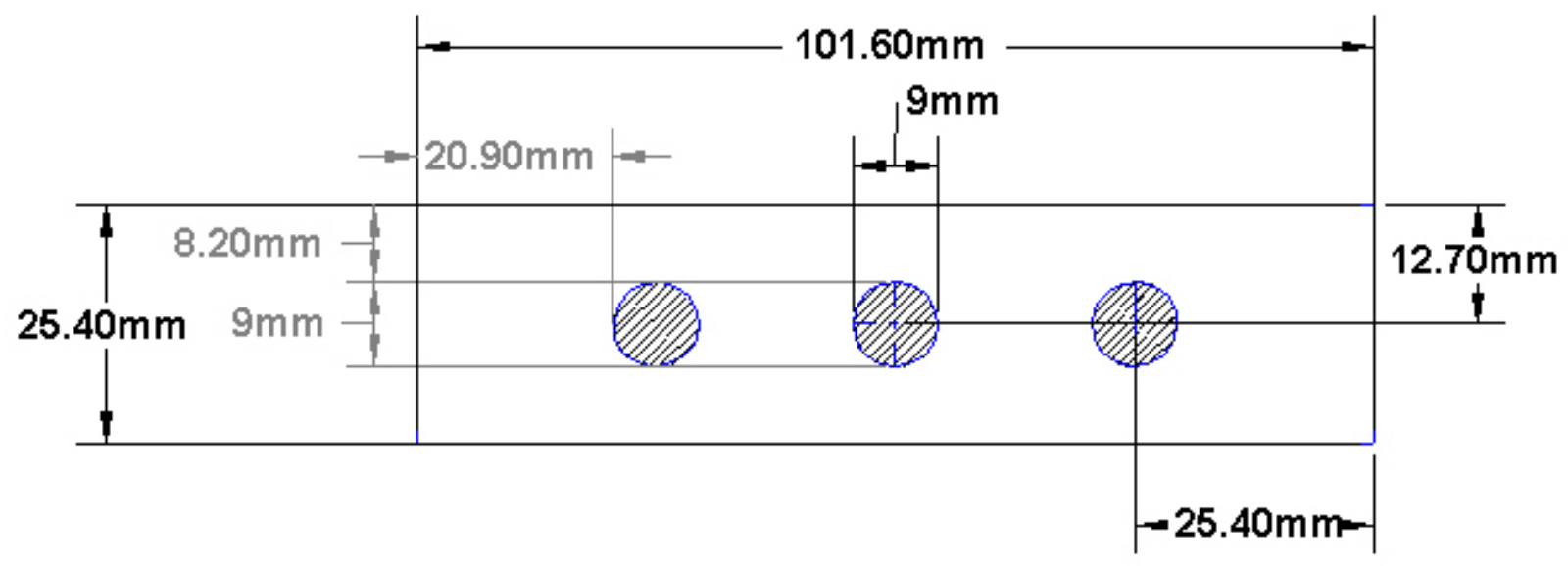

Figure 64 - Schematic of the three large via cell: 3.9. 


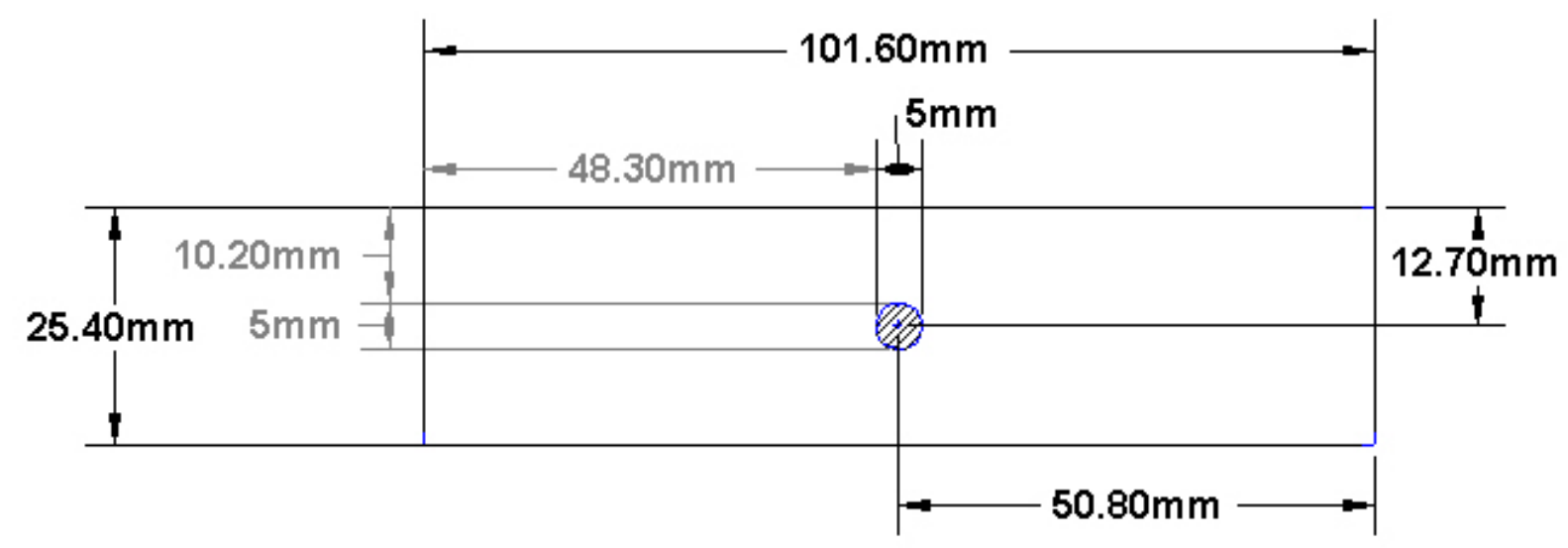

Figure 65 - Schematic of the one small via cell: 1.5 .

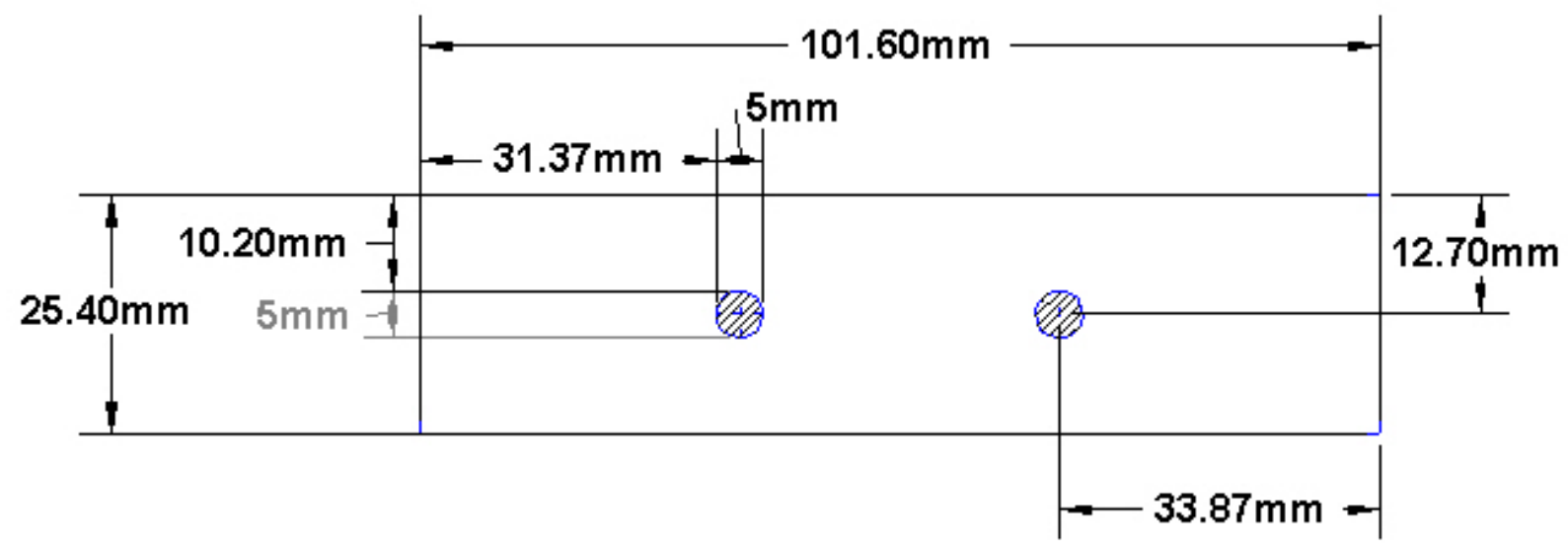

Figure 66 - Schematic of the two via cell: 2.5.

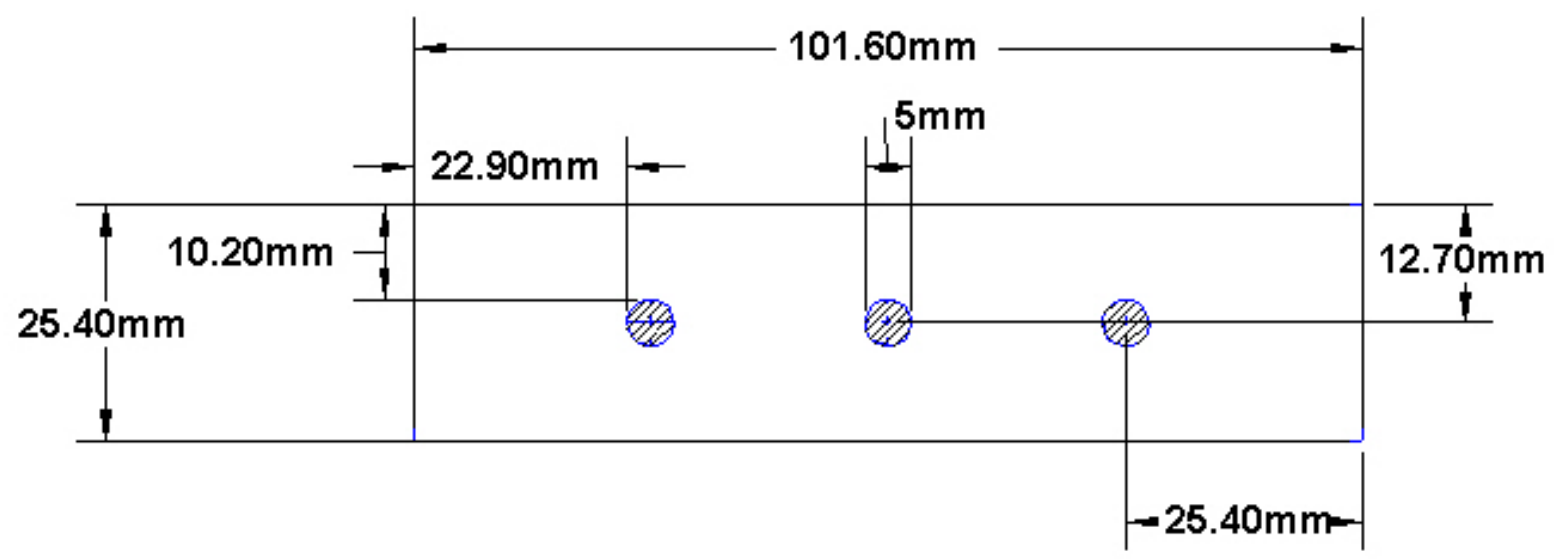

Figure 67 - Schematic of the three via cell: 3.5 . 


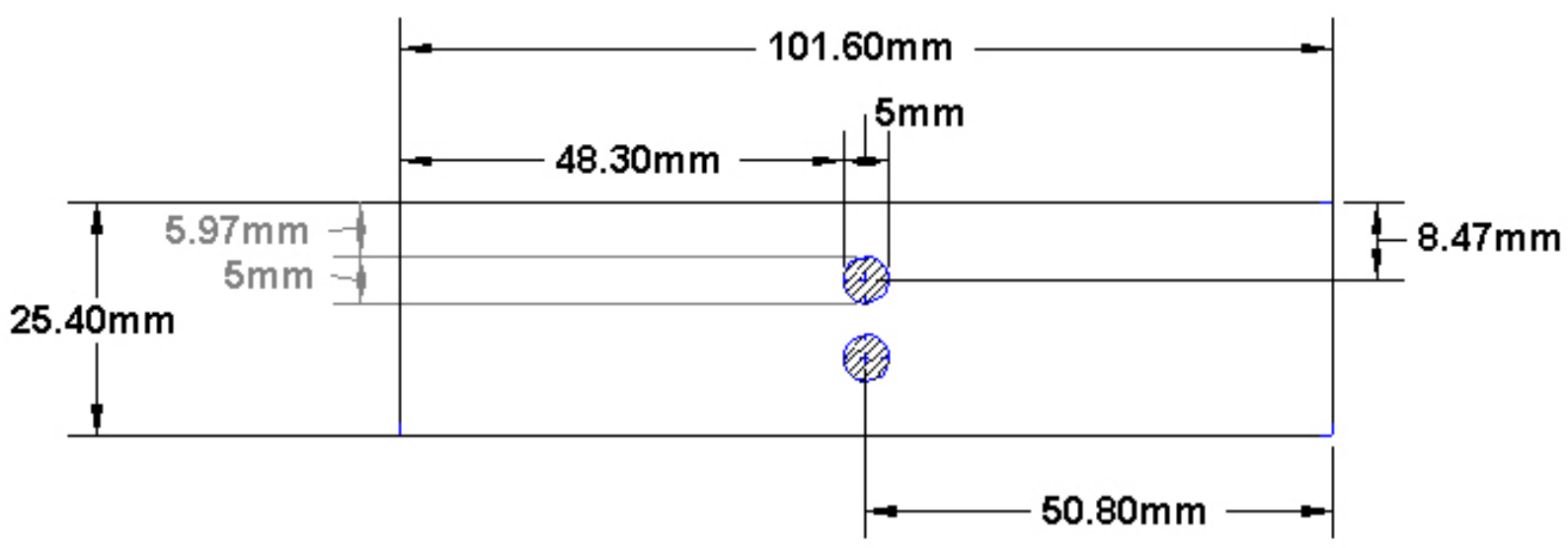

Figure 68 - Schematic of the one, paired small hole cell: 2.55.

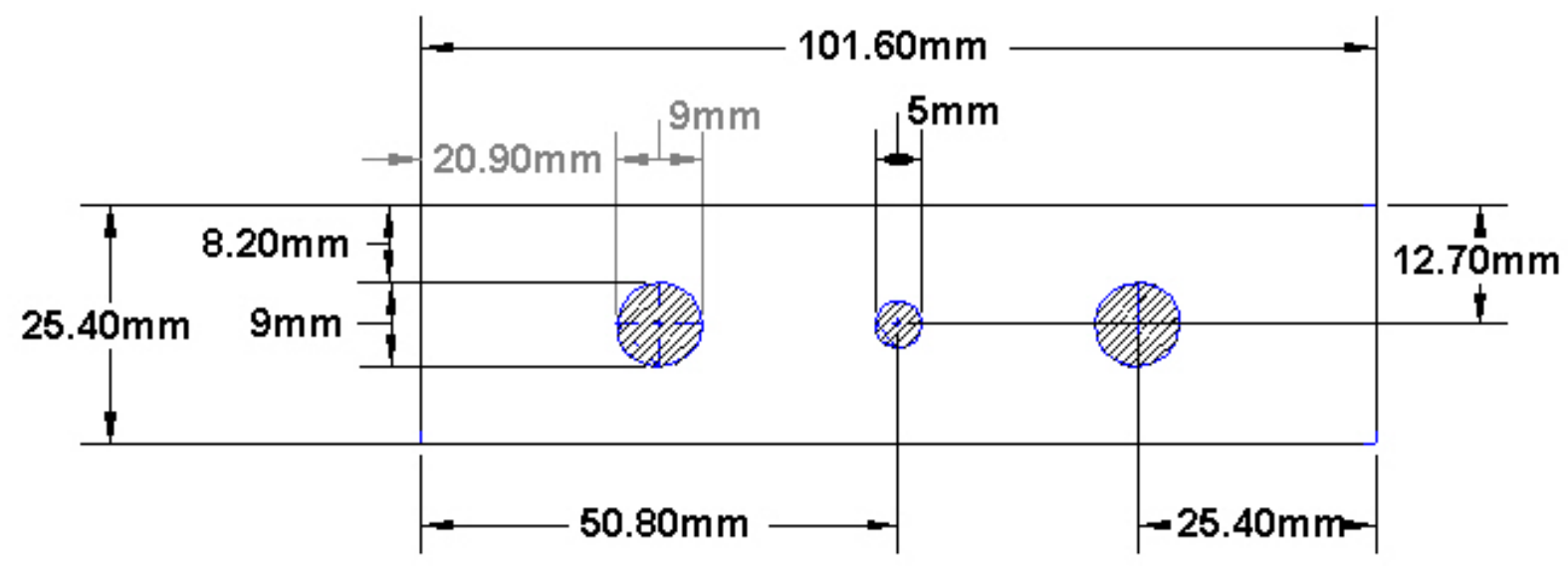

Figure 69 - Schematic of the three via mixed cell: 3.959.

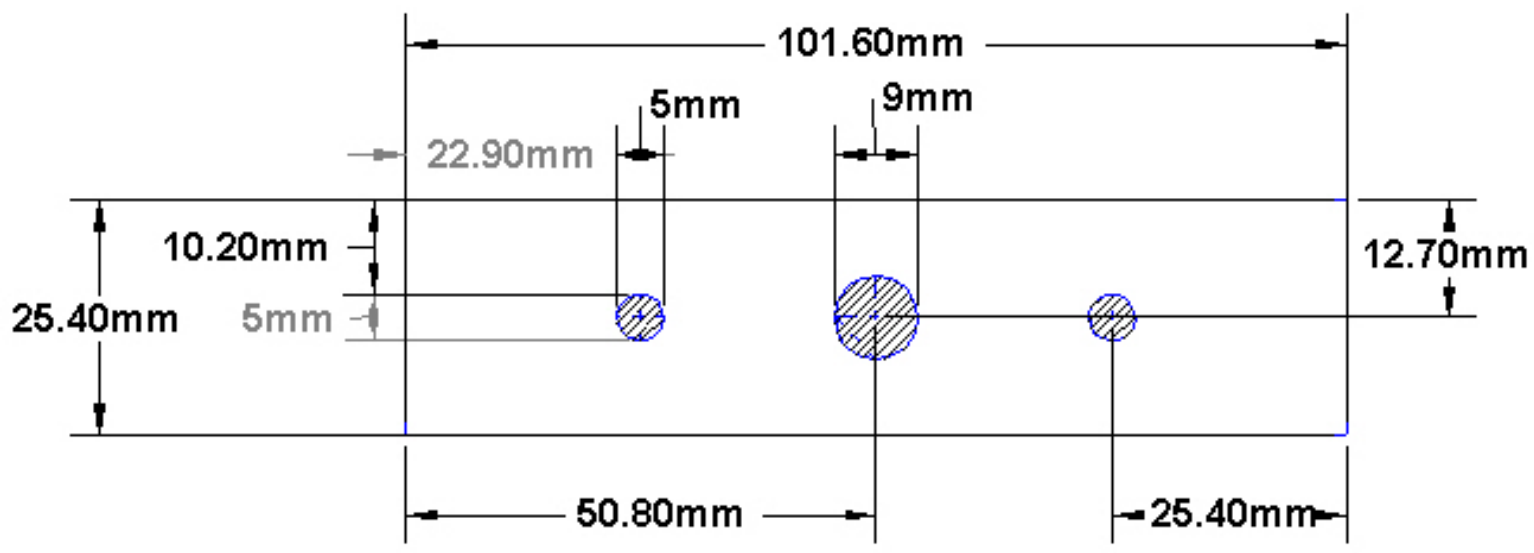

Figure 70 - Schematic of the three via mixed cell: 3.595. 


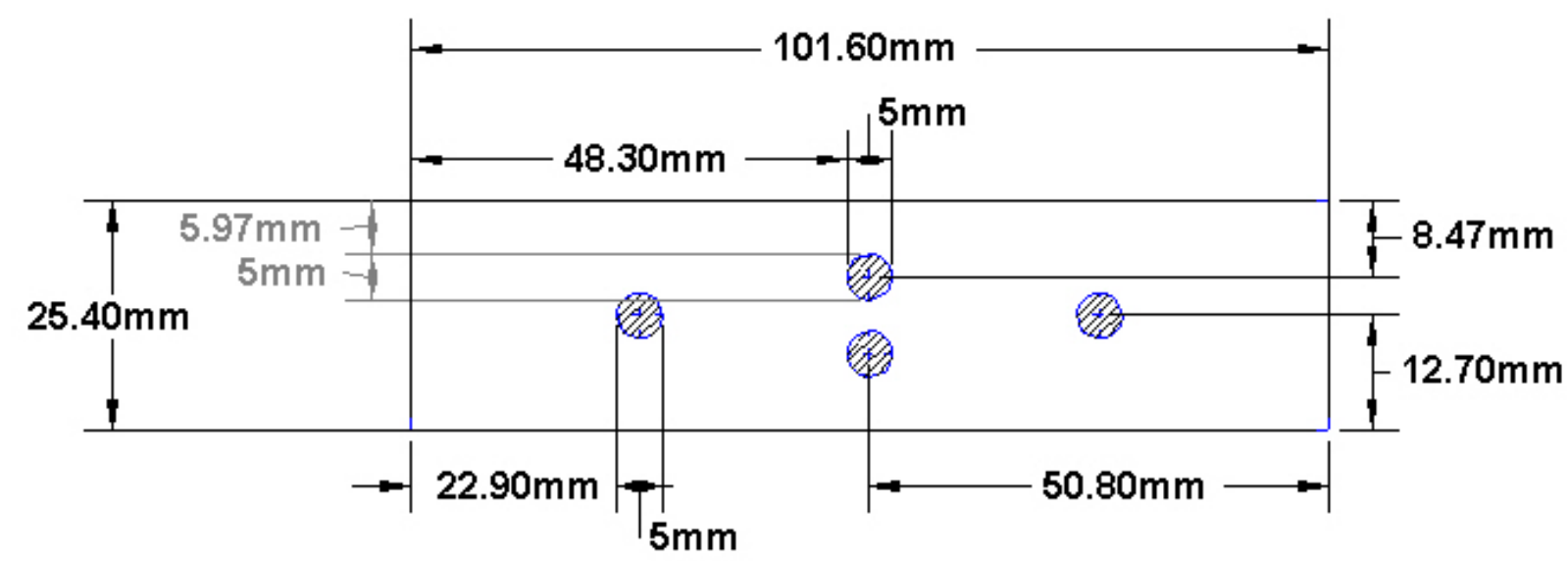

Figure 71 - Schematic of the four via diamond cell: 4.5 .

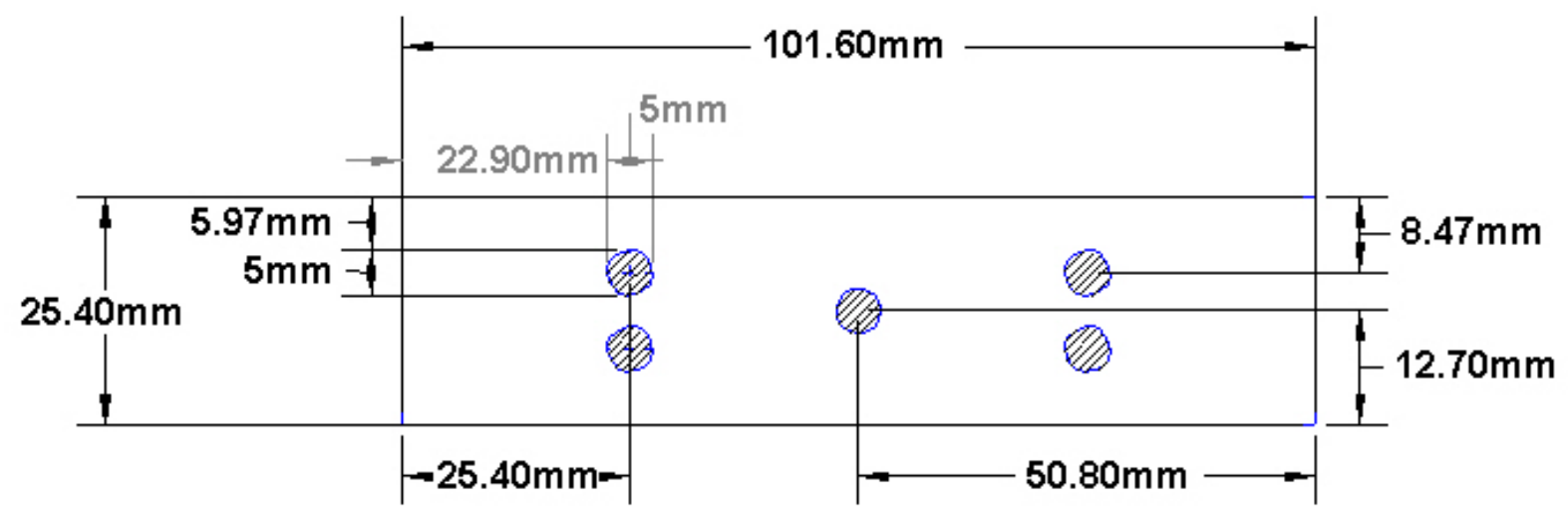

Figure 72 - Schematic of the five via diamond cell: 5.5 .

These configurations were based on a combination of two different diameter vias distinctly designed and implemented for each proposed structural battery to increase the mechanical strength. The designs included linear, as well as a pairing of the perforations to determine how each diameter and specific placement geometry increased the overall mechanical properties. The increase in delamination strength was then directly related to the remaining useable battery area to extrapolate the best design for fabrication of different object specific shapes as structural batteries.

Each via creates a channel for the epoxy matrix when being fabricated within a composite object, which is the key point of the design of a formable structural battery. These reinforcement vias pass through the active components within a pouch battery, allowing for shear reinforcement throughout the interior of the battery compartment. 
Although vias reduce the overall capacity, they permit the ability to customize the shape of the battery. Consequently, this eliminates the requirement of designing components around a traditional battery and allows for integration of the power supply throughout the structural component.

\subsubsection{Fabrication}

The carbon fiber utilized for fabrication of structural batteries in this testing was a PAN-based $1 \mathrm{~K}$ plain carbon fiber weave, with a light $118.7 \mathrm{~g} / \mathrm{m}^{2}$ weight, and a $178 \mu \mathrm{m}$ thickness (CF14X, The Composites Store). Samples were fabricated based on $0^{\circ} / 90^{\circ}$ two ply orientations, with the structural battery architectures employed as the core. The samples were fabricated by a vacuum infusion technique that allowed the assembly of the carbon fiber mats and all of the core structural battery architectures in a single step. The epoxy system employed for this fabrication was the 635 thin epoxy resin system, with the corresponding 556 slow epoxy hardener (US Composites). Selection of this particular system was based on the desired long working time of 40 minutes and the low viscosity of $600 \mathrm{cps}$. This permitted an even flow of the epoxy throughout the entire layup based on the employed vacuum infusion technique.

The vacuum infusion technique used vacuum pressure to drive the epoxy resin into the composite materials. This allows for all of the dried materials to be properly layered together where vacuum pressure is then applied upon the materials, allowing for adjustments to any of the individual components before any resin is introduced to the system. Once a full vacuum is achieved, the resin is forced through the layup via vacuum tubing. This process results in a much better fiber to resin ratio than the hand layup process, which introduces the epoxy resin to each layer as it is being fabricated [93], [94].

This in-house fabrication of manufacturing structural batteries for mechanical testing began with cutting the cathode (aluminum) and anode (copper), $50.8 \mu \mathrm{m}$ thick, foils to form the current collector into the designed shape. The vias were carefully measured and marked for each structural architecture on the foils where they were each precisely produced with a round hole arch punch. These foils were layered together with 
a tri-layer polypropylene/polyethylene/polypropylene (PP/PE/PP) separator membrane (Celgard, 2325) between them to mimic the internal components of a lithium ion battery. These components were then stacked between two $127 \mu \mathrm{m}$ thick pieces of an ultra-high molecular weight polyethylene (UHMW-PE) film (CS Hyde Company). This film was selected due to the chemical resistance and physical makeup of PE not affecting the chemical reactions within a functioning lithium ion battery. Also, the low melting point of $\mathrm{PE}$ allowed for a heat sealing technique of the material without any effect to the internal battery components. Once layered, the PE was completely heat sealed around the edges of the internal battery components and between each manufactured via. A smaller round hole arch punch was then utilized once again to produce a slightly smaller hole through the sealed PE in each via to create an open channel for the epoxy matrix to occupy during vacuum infusion.

The initial step for the composite manufacturing was to measure and cut all of the required materials to length with a rolling blade on a cutting board (carbon fiber, release film, perforated mesh film, and breather cloth). The fabrication layup of these structural batteries were conducted on a sheet of glass starting with a light layer of wax, to prevent sticking and allow for an easy release of the composite layup once the epoxy hardens (Figure 73). The first material layer on the wax coated glass was a $0^{\circ} / 90^{\circ}$ oriented carbon fiber fabric, next were the sealed structural battery samples evenly spaced throughout the fabric, followed by another layer of $0^{\circ} / 90^{\circ}$ oriented carbon fiber fabric. These materials were all of the materials required for the laminate structural batteries; the rest were purely utilized for fabrication purposes. 


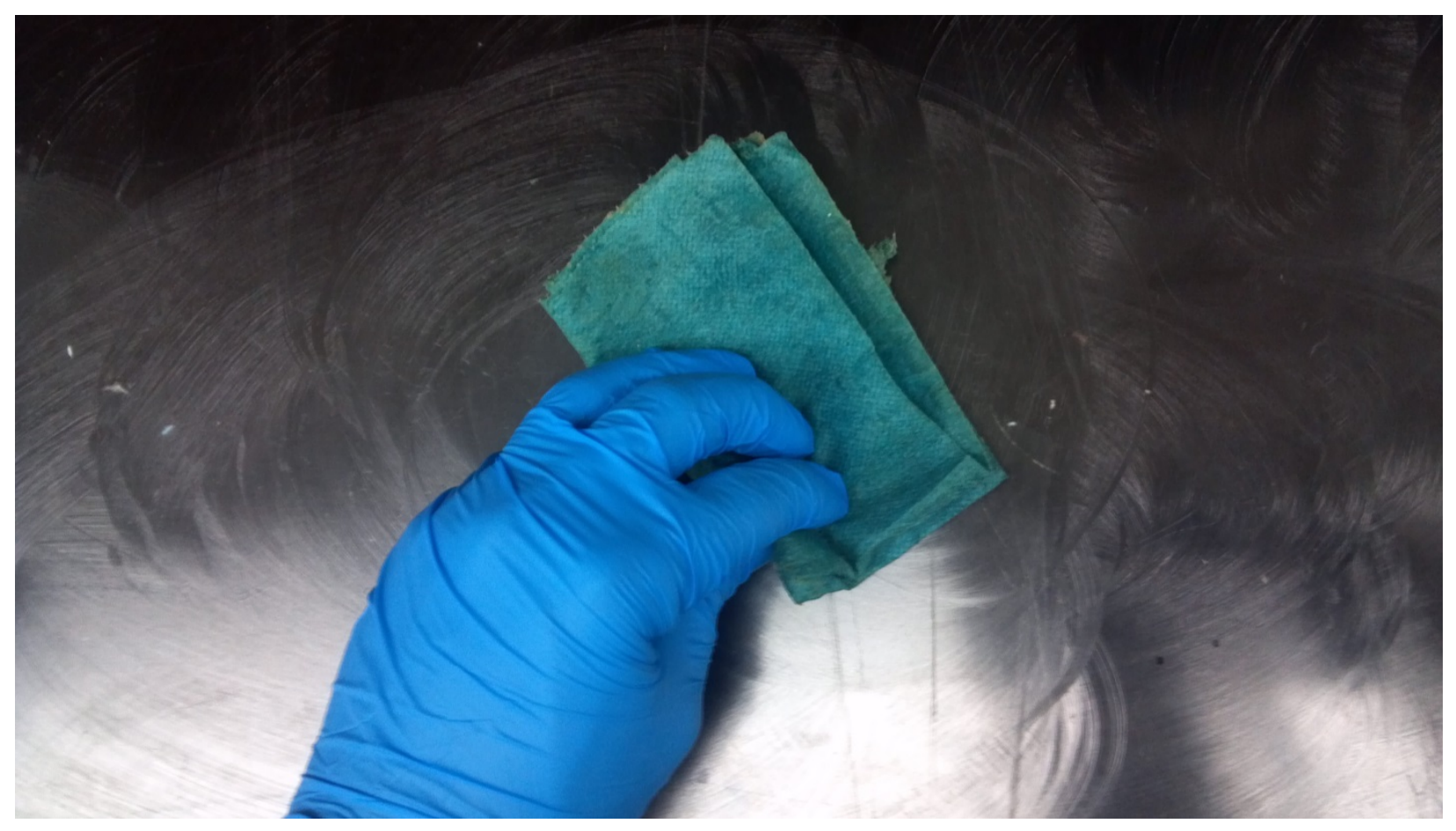

Figure 73 - Picture of applying a thin layer of paste wax, with cloth, onto the glass sheet.

The first fabrication layer on top of the composite sample was a perforated release film known as peel ply. This nylon micro-porous layer is designed to not bond with epoxy, while allowing the flow of epoxy resin through it during vacuum infusion. Once the epoxy was cured, the release film easily peeled off of the fiber laminate and resulted in a smooth textured surface. The next layer placed above the release film was a perforated mesh film. This layer provided pathways to assist in an even distribution of epoxy, in addition to holding resin in the laminate under vacuum. The last material was a polyester breather cloth that allowed air to be distributed throughout the entire setup. Also, this material absorbed excess epoxy during infusion to ensure an even distribution of resin was achieved.

The next step in the vacuum infusion process was a layer of sealant tape used to surround all of the materials and the waxed surface. Evenly spaced around the layup were vacuum tubes with sealant tape wrapped around them in order for the epoxy to be infused during vacuum on the setup. A channel for the vacuum to be pulled was laid through the middle of the sample with the breather cloth surrounding it to prevent epoxy from entering the vacuum system, as well as, providing an even distribution for the 
vacuum to be pulled. The top layer covering all of the materials is a $76.2 \mu \mathrm{m}$ thick polyethylene thin film that is attached to the sealant tape surrounding the composite layup, which allowed for vacuum to be pulled throughout the entire setup at once [94], (Figures 74 and 75).

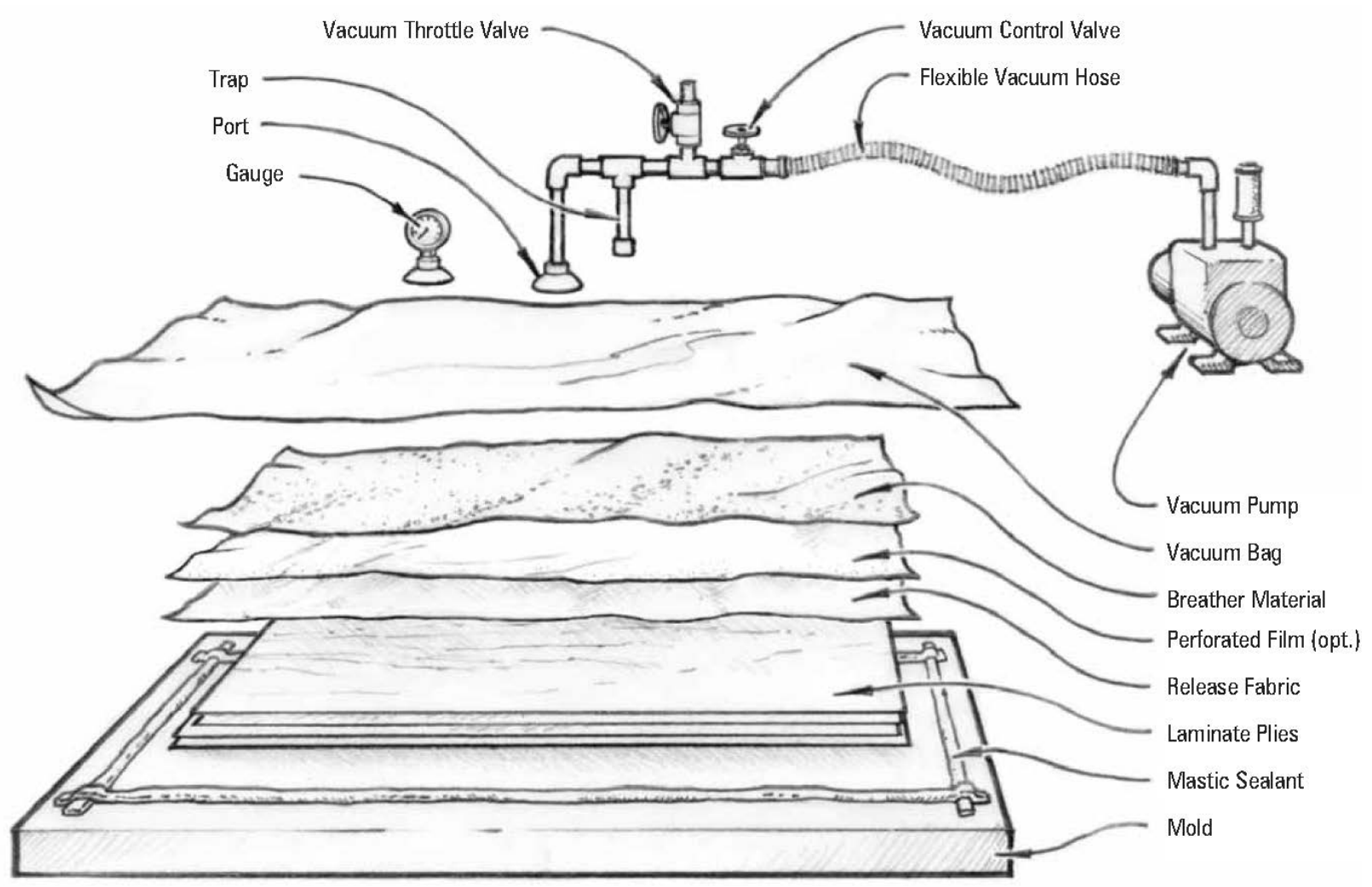

Figure 74 - Schematic of typical components utilized in a vacuum forced epoxy infusion system [94]. 


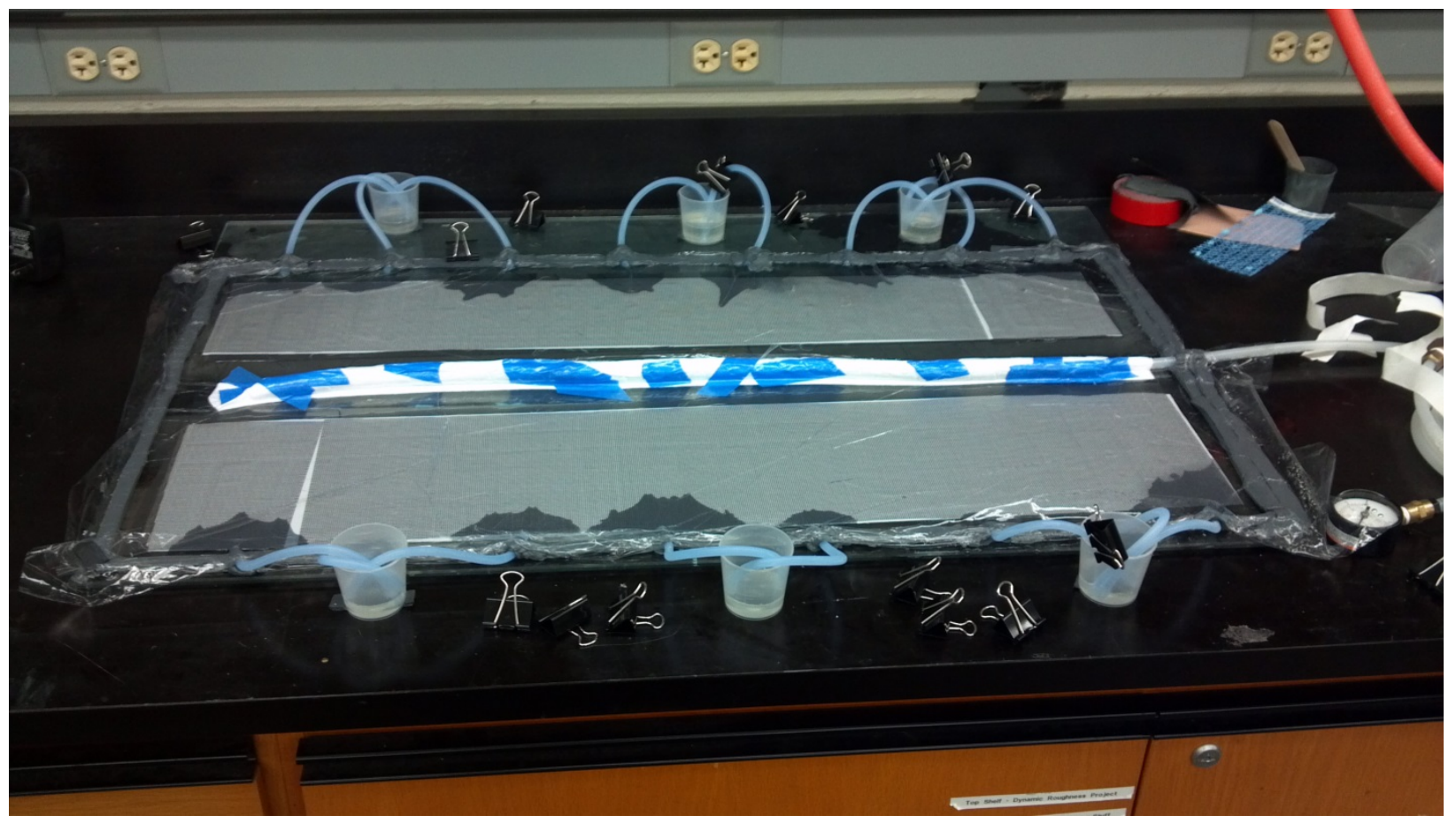

Figure 75 - Picture of in-situ epoxy flow during vacuum forced infusion on composite batteries.

Once all of the materials were in place, all of the vacuum tubes are clamped off to create a completely sealed off system where the vacuum was then introduced and the setup was checked for leaks. After a full vacuum was achieved $(\sim 18 \mathrm{inHg})$, a large batch of epoxy was synthesized at a 2:1 resin to hardener ratio and transferred to smaller cups. The vacuum lines are then inserted into the mold and the vacuum infusion process is initiated. This procedure required about 30 minutes for full infusion where the lines were once again clamped off and the layup was left to dry until cured for a full 24 hours. The next step was to remove the fabrication only materials leaving a sheet of $0^{\circ} 190^{\circ}$ oriented two ply carbon fiber with the structural core battery samples inside. The structural batteries were measured and cut to size with serrated scissors where they now were ready for mechanical analysis.

\subsubsection{Four Point Beam Analysis}

The protocol followed for this testing was ATSM Standard D6272-10, which is for "Flexural Properties of Unreinforced and Reinforced Plastics and Electrical Insulating Materials by Four-Point Bending". This standard called for a sample with dimensions of $101.6 \mathrm{~mm}$ in length by $25.4 \mathrm{~mm}$ in width across a $50.8 \mathrm{~mm}$ support span. The 
crosshead rate was dependent on the thickness of the sample being tested, which was consistent between all of the composite materials and was determined by:

$$
\text { Eq. } 4-R=0.167 Z^{2} / d
$$

Where $R$ is the rate of the cross motion [ $\mathrm{mm} / \mathrm{min}], L$ is the support span $[\mathrm{mm}], d$ is the thickness of the sample [mm], and $Z$ is the straining rate of the outer fibers. Five samples of each simulated multifunctional battery were examined in each test as required by ASTM standards.

The testing machine utilized for conducting the four-point bending was an Instron model 3365 universal test machine. The apparatus for testing was manufactured inhouse according to ASTM specifications for a one half support span, four-point bending protocol. This protocol calls for a beam that is loaded in flexure at two central points and supported at two outer points. The maximum stress occurs in the fibers between the two central loading points that define the load span. The stress is than able to be calculated for any point during the deflection by:

$$
\text { Eq. } 5 \text { - } S=3 P L / 4 b d^{2}
$$

Where $S$ is the stress in the fiber throughout the load span [MPa], $P$ is the load at a given point [N], $L$ is the support span [mm], $b$ is the width of the beam [mm], and $d$ is the depth of the beam [mm]. 


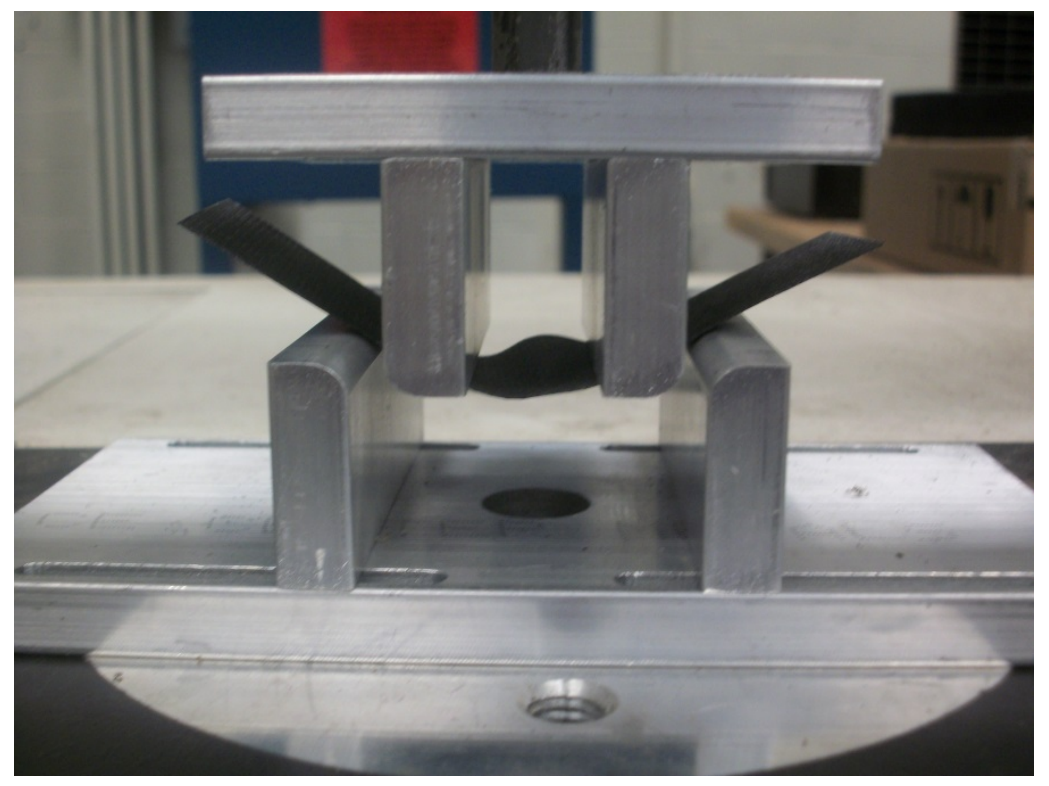

Figure 76 - Delamination occurring in mechanical samples during four point bending.

The point of delamination is identified as the point where the battery would cease to fully function electrochemically and the carbon fiber structure began to separate; however, significant strength of the composites typically remained even after failure. The tests were conducted at the determined rate until delamination occurred in each sample recording the load required for the deflection every second. The stress for each test was calculated at every increased deflection point until delamination of the sample occurred (Figure 77). 


\section{Strength of Proposed Structural Battery Configurations}

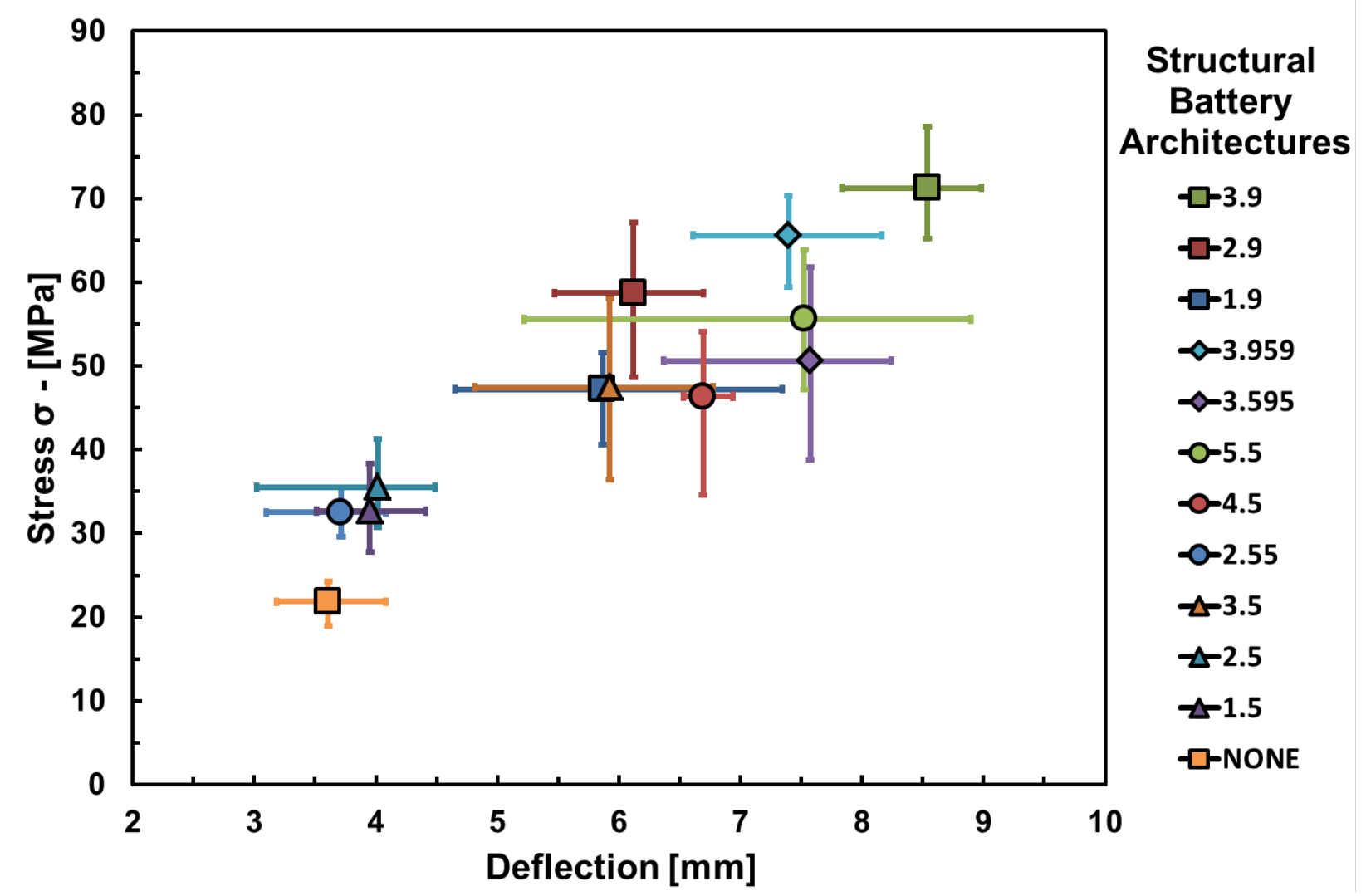

Figure 77 - The stress and deflection ranges of the different proposed structural battery architectures.

This analysis exemplified how the architecture of each structural battery affected inherent mechanical properties. For each series of five samples, the average stress was graphed at the average deflection point where delamination occurred. Error bars were also incorporated to display the range of all five samples, in regards to their stress and deflection delamination points. Additionally, the loss of functional battery area from each design, resulting from the manufactured vias, is another characteristic that was compared to the corresponding strength (Table 10). 
Table 10 - Functional area of structural battery architectures in regards to strength of architecture before flexure before delamination.

\begin{tabular}{|r|r|l|l|}
\hline $\begin{array}{l}\text { Battery } \\
\text { Core } \\
\text { Architecture }\end{array}$ & $\begin{array}{l}\text { Functional } \\
\text { Battery } \\
\text { Area [\%] }\end{array}$ & $\begin{array}{l}\text { Average } \\
\text { Flexural } \\
\text { Strength } \\
\text { [MPa] }\end{array}$ & $\begin{array}{l}\text { Average } \\
\text { Delamination } \\
\text { Deflection } \\
\text { [mm] }\end{array}$ \\
\hline None & 100.0 & 21.86 & 3.61 \\
\hline 2.55 & 98.5 & 32.52 & 3.71 \\
\hline 1.5 & 99.2 & 32.66 & 3.95 \\
\hline 2.5 & 98.5 & 35.50 & 4.02 \\
\hline 3.5 & 97.7 & 47.43 & 5.93 \\
\hline 4.5 & 97.0 & 46.37 & 6.69 \\
\hline 5.5 & 96.2 & 55.57 & 7.53 \\
\hline 3.595 & 96.0 & 50.62 & 7.57 \\
\hline 3.959 & 94.3 & 65.58 & 7.40 \\
\hline 1.9 & 97.5 & 47.21 & 5.87 \\
\hline 2.9 & 95.1 & 58.71 & 6.12 \\
\hline 3.9 & 92.6 & 71.27 & 8.54 \\
\hline & & & \\
\hline
\end{tabular}

The common trend for all of the simulated composite battery architectures showed an overall steady increase in strength, while more of the functional battery area was removed for the existence of an epoxy matrix through perforated vias. The average rate of this observed strength increase throughout the different structural architectures was $6.6 \mathrm{MPa}$ for a $1 \%$ contribution from the useable battery area to the epoxy matrix. Similarly, the flexibility of the different battery designs, before delamination, also increased at an average rate of $1.11 \mathrm{~mm}$ for every $1 \%$ loss of functional battery area. This substantial strength increase was manifested in the reduction of delamination and wrinkling incidences within the multifunctional battery architecture.

The purpose of this evaluation was to provide the ability of selecting an optimal core architecture best designed for an object specific multifunctional battery. Selection criteria are based on the resulting components required strength, rigidity, and required power.

\subsubsection{In-Situ Multifunctional Battery Four Point Beam Analysis}

To truly complete this evaluation, a functioning multifunctional battery based on one of the proposed core architectures was manufactured and analyzed. Other groups 
have performed mechanical integrity testing on pouch type and cylindrical type batteries. Their respective mechanical investigations have composed of: compression between flat plates, indention, four bar shear, three and four point bending. Furthermore finite element simulations were typically conducted for each of their specific tests [95], [96]. One group performed indentation testing on both types of these cells, while measuring voltage and temperature, as the battery experienced mechanical deformation until failure [97]. Furthermore, another group investigated the capacity fade observed while discharging pouch cells under a constant compressive stress [29]. However, in-situ electrochemical performance analysis with correlating mechanical integrity testing had not been performed.

The main approach performed to improve the multifunctional performance, by other groups, consist of embedding commercial lithium ion pouch cells within different structural panels. Subsequently, other groups are working on ways to improve the mechanical characteristics of the actual electrodes in order to improve the battery's mechanical properties [1], [10], [60].

The architecture selected for an in-situ combined electrochemical and mechanical performance test was the 1-9 large via cell. This architecture was selected due to resulting strength and deflection properties range was near the middle of all the proposed battery designs. Fabrication of this cell was conducted similar to the previous protocol, with the addition of cutting tabs on the foil current collector for electrochemical testing, and injection the required liquid electrolyte before complete sealing of the battery. The testing protocol of this cell began with a C/25 SEI formation of 1.5 cycles. This was followed by a typical $\mathrm{C} / 2 \mathrm{CC}$ discharge to $3.0 \mathrm{~V}$ with a $\mathrm{C} / 2$ to $\mathrm{C} / 20 \mathrm{CC} / \mathrm{CV}$ charge to $4.2 \mathrm{~V}$ to show the expected performance of this structural cell.

The next step consisted of a $\mathrm{C} / 2$ discharge while simultaneously conducting four point beam deflection testing to determine the durability of the structural cell as it begins to fade until failure (Figure 78). Mechanical testing was initiated once the battery approached 3.8 $\mathrm{V}$ to ensure a stable electrochemical discharge rate. As pressure was applied to the battery, the voltage steadily rose continuing to function past the point of delamination to the outer layered carbon fiber composite laminate (Figure 79). Once the 
mechanical cycle was completed the voltage dropped instantly back down to $3.77 \mathrm{~V}$. Since the battery did not experience a catastrophic failure, the electrochemical test continued while repeating in-situ mechanical analysis at $3.7 \mathrm{~V}, 3.6 \mathrm{~V}, 3.5 \mathrm{~V}$, and $3.4 \mathrm{~V}$ (Figure 80). The multifunctional cell continued a discharge to $3.0 \mathrm{~V}$, where it was fully cycled once more to determine if it was still functional after mechanical deformation (Figure 81).

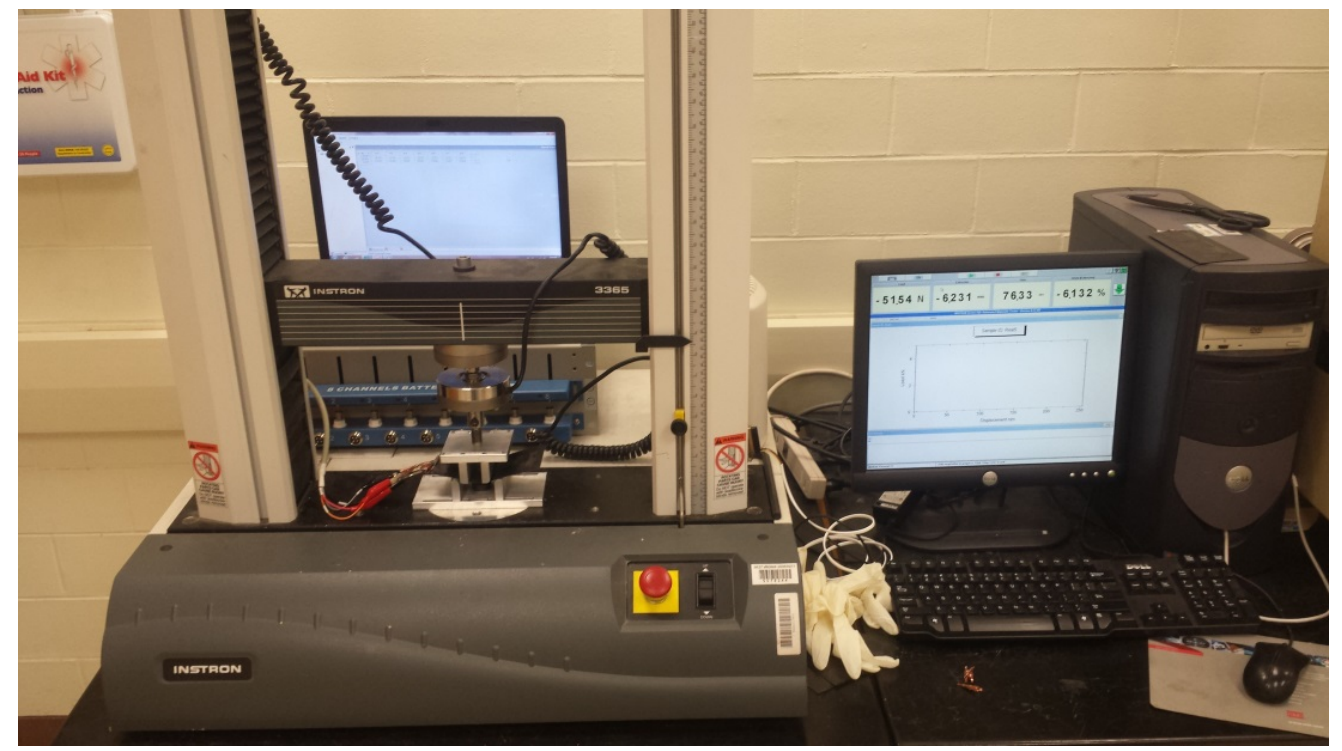

Figure 78 - Picture of in-situ multifunctional battery analysis equipment.

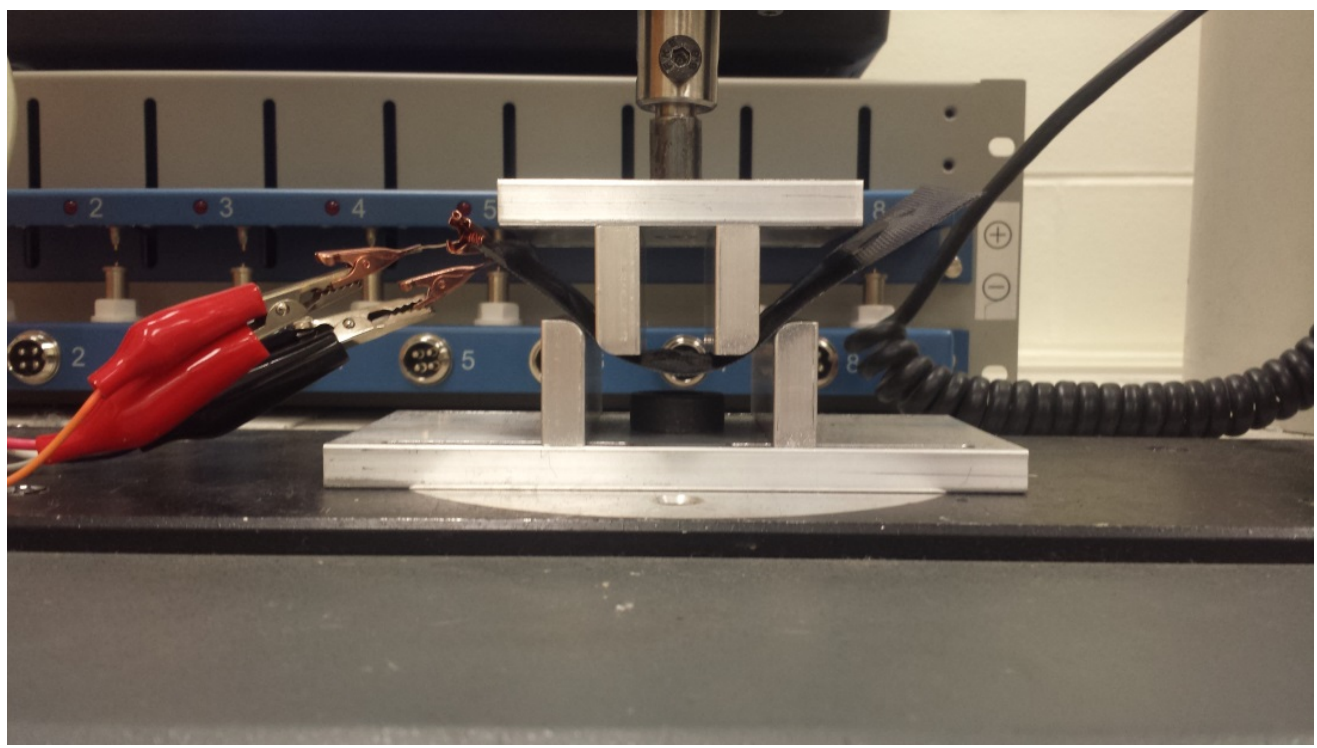

Figure 79 - Picture of in-situ multifunctional battery composite laminate delamination, while electrochemically tested under mechanical four-point beam load. 


\section{Structural Cell 1-Via In-Situ Four Point Bending Durability}

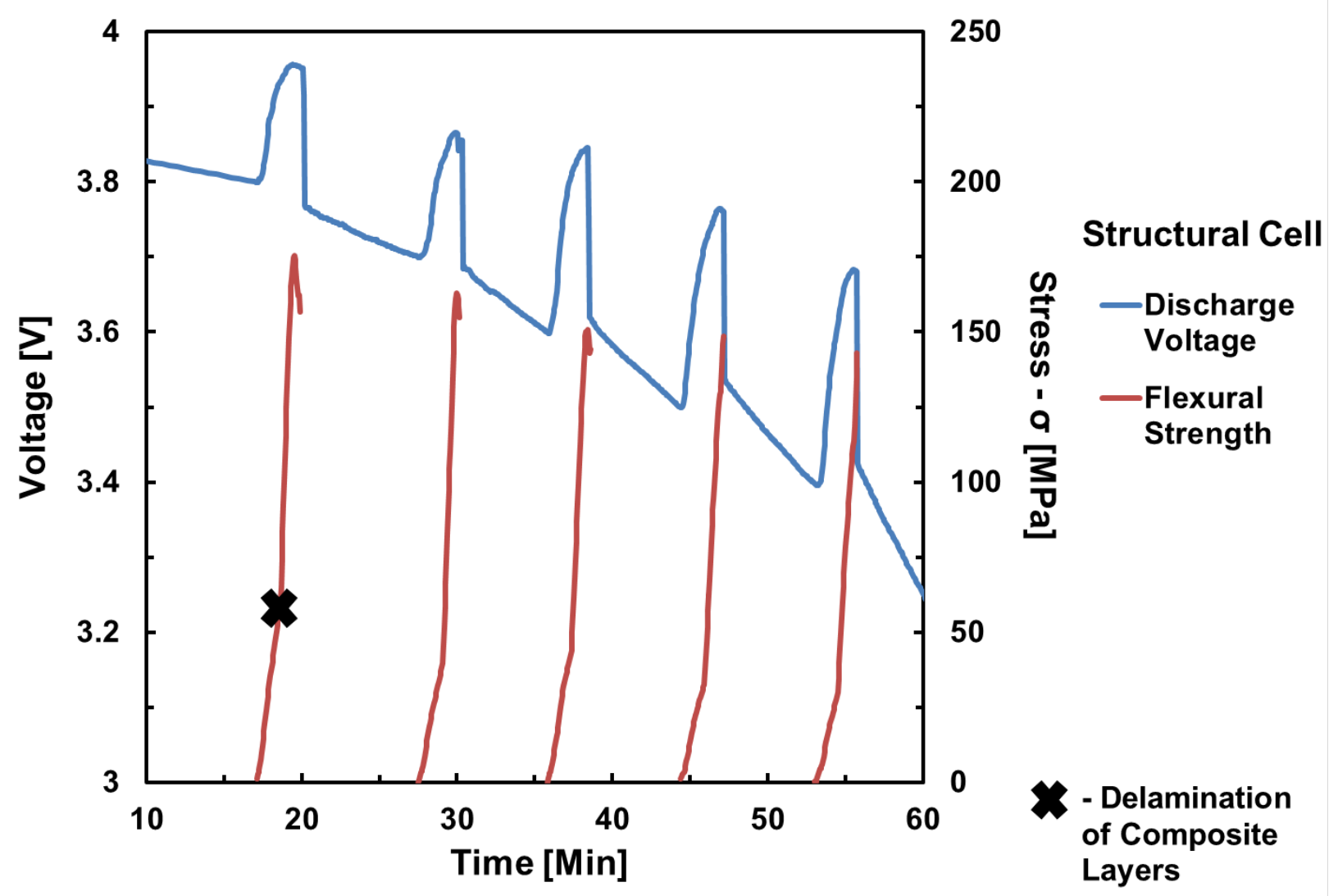

Figure 80 - The in-situ analysis of a 1.9 large via multifunctional battery voltage, during a $\mathrm{C} / 2$ discharge rate, aligned with corresponding four-point beam strength evaluations. 


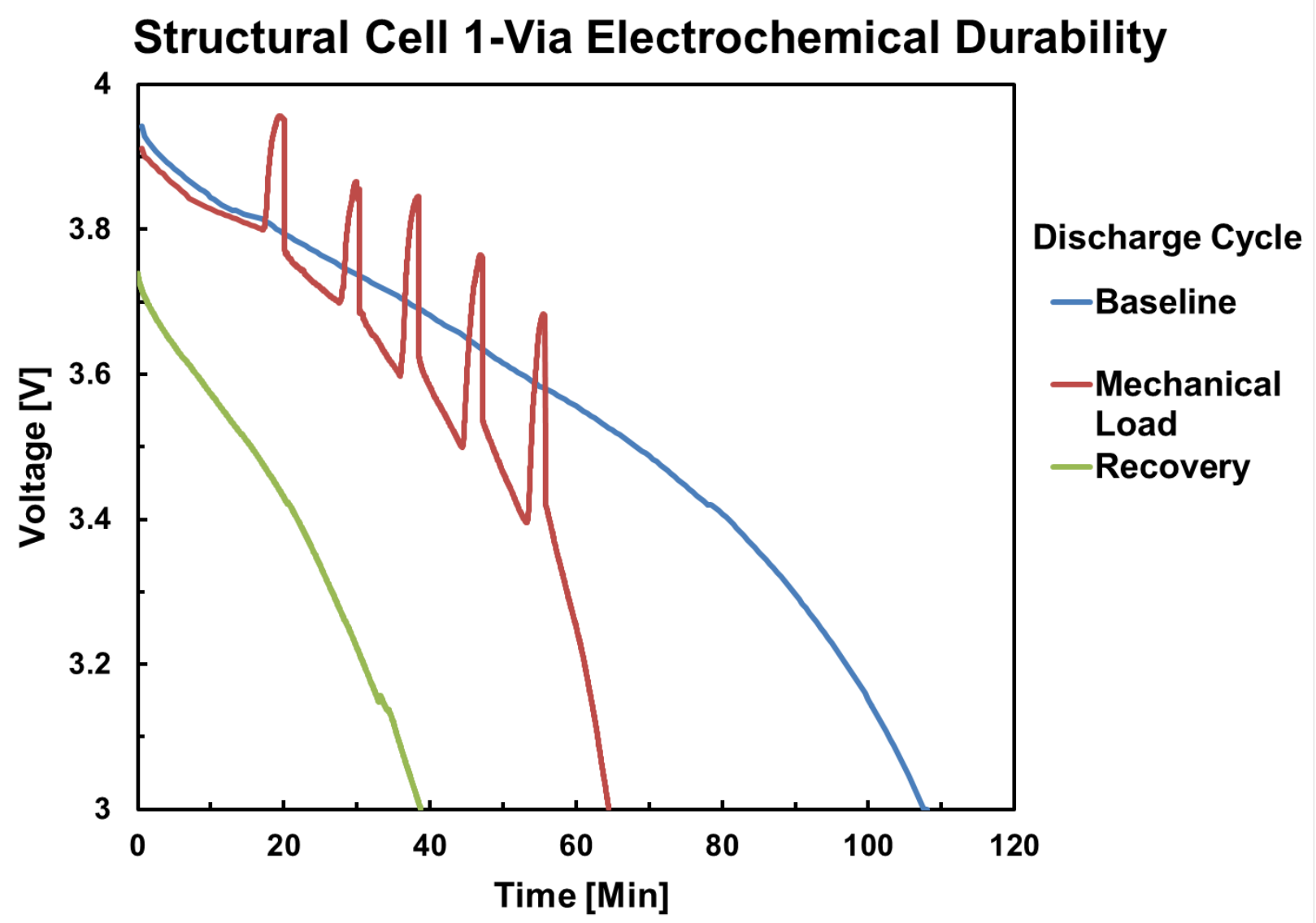

Figure 81 - Electrochemical performance analysis of the 1.9 large via designed multifunctional battery: before, during, and after mechanical deflection investigations.

The electrochemical performance obtained from the multifunctional battery in-situ mechanical strength investigation was revolutionary. A complete delamination and electrochemical failure of the internal battery components was anticipated to occur at the strength where shear delamination was transpired to the composite laminate layers. Electrochemical failure was also proposed to occur attributed to fracturing of the heat sealed UHMW-PE battery case while under mechanical loads. Since an internal short circuit of the battery was expected, that can lead to thermal instability, electrochemical loading was programmed to terminate if the voltage drop was sudden and uncharacteristic. However, the battery did not experience complete failure under the imposed mechanical loading and continued to function, at a reduced efficiency, even after five mechanically induced deformations. Furthermore, a significant strength of the multifunctional battery remained even after shear failures. An increase of voltage was 
also observed during each mechanical cycle; once complete, the voltage dropped and the battery continued to discharge at a steady rate.

The multifunctional battery experienced a capacity loss of $37 \%$ during to the imposed mechanical forces, and another $41 \%$ loss after deformation. This high loss is contributed to soft shorting; this failure method is irreversible and is due to very small localized contacts of the electrode layers through the separator due to mechanical deformation [97]. The retained strength of the multifunctional battery is due to the type of mechanical failure observed in the composite laminate structure. Only a skin compressive failure of the laminate layers occurred during deflection, whereas a core shear failure of the epoxy matrix filled via did not occur [98], [99]. The observed failure allowed the battery to retain a high level of rigidity, experiencing an $18 \%$ deterioration of strength throughout repeated mechanical measurements.

Lastly, the voltage increase observed under mechanical loading, was not documented throughout literature in any mechanical evaluations to batteries. This

phenomenon is believed to have occurred due to the increasing mechanical pressure on the battery shrinking available pore volume in the micro-porous separator [29]. As more of the pathways for ionic transport were blocked, the potential energy of the battery temporarily increased as a result of a greater separation in the electrically charged electrodes.

\subsection{Discussion}

The ultimate goal of this section was to conduct a mechanical evaluation of several different architecturally modified lithium ion pouch batteries. Perforations were created at different structural points throughout the battery, with the purpose to increase bending strength and reduce delamination of internal components. This evaluated modification created formable multifunctional structural batteries, which when layered within a composite matrix enhanced the normal and shear strength.

The data interpreted from this chapter allows for the design of object specific structural batteries, based on the required strength and electrochemical output of the structural component. These requirements determine the architecture necessary for 
fabrication of an optimal battery for specific applications. Furthermore, this proposed type of architecture modification to the classical cell architecture was proved to be possible and effective through in-situ electrochemical and mechanical investigations to an in-house manufactured multifunctional battery. Although this mechanical data is from single cell batteries, more than one cell can be layered within the structure and interconnected to increase the voltage or capacity of the cell depending on the desired application. These designs allow for the ultimate optimization of each fabricated cell to be specifically tailored to the demand for its application. 


\section{Chapter 6: Conclusions and Future Work}

The aim of this research was to design and test two composite multifunctional battery architectures that can serve as a structure as well as the power supply for a structural component. The work first focused on the cyclic voltage-capacity testing of the first battery architecture (Figure 19). Several differently pretreated carbon fiber composite fabrics were substituted as the anode material, where they underwent the same electrochemical protocol and their resulting performance was compared. The second part of this work centered on the mechanical testing of the second battery architecture (Figure 20). This second architecture consisted of the modification of a conventional multifunctional lithium ion pouch batteries design by creating perforations through the cell which develops a formable multifunctional battery with enhanced mechanical strength.

The electrochemical testing of the first battery architecture was conducted in coin cells. Once a baseline cell was established that demonstrated a $5.7 \%$ capacity loss due to cycling, pretreated carbon fiber composite materials were directly substituted for the anode component. There was a $42.5 \%$ relative capacitance loss due to the substitution of an untreated carbon fiber fabric anode, whereas a reducing atmospheric pretreatment at $400^{\circ} \mathrm{C}$ positively affected the surface of the carbon fiber anode resulting in only a $23 \%$ loss of capacitance over cycling. Further analysis of pretreatments to other carbon fiber fabrics confirmed that the designated pretreatments similarly affected their electrochemical performance. This testing proved that carbon fiber is a viable alternative battery material, despite lower electrochemical performance rates.

Future electrochemical analysis would include a more in-depth investigation of the pretreated carbon fiber materials. An XPS deconvolution of the $\mathrm{O}$ 1s and $\mathrm{N}$ is peaks could further define surface functional groups on the fibers. X-Ray diffraction characterization should also be incorporated to determine if the different pretreatments altered the physical structure of the fibers. Additionally, tensile testing would determine structural deterioration of the fiber strands. Different electrolyte systems should be implemented to determine if other carbonate solutions would react differently with the modified surface functional groups; therefore, possibly lowering the initial irreversible 
capacitance loss and resulting fade throughout cycling. Lastly, a more thorough evaluation on the effects of different composite separator materials should be initiated in order to determine the best performing combination of composite materials that can be applied to the prospective goal.

The electromechanical testing of the second battery architecture showed that the perforated battery pouch structure enhanced the normal and shear strength of the composite structure. In-situ electromechanical four point beam analysis of a modified architecture experienced skin compressive failure of the laminate layers during deflection, whereas a core shear failure of the epoxy matrix filled via did not occur. However, the multifunctional battery did experience a capacity loss of $37 \%$ during imposed mechanical forces, and another $41 \%$ loss after deformation to the structure. The evaluation from this approach can be applied to the prospective goal of fabricating a high capacity composite multifunctional battery with enhanced strength by utilizing an epoxy matrix through the cell at key structural points.

A future electromechanical analysis would entail the fabrication of complex architectural multifunctional batteries. Further in-situ testing, to determine where internal irreversible soft shorting initiates, would determine the true durability of the proposed battery architectures. Also, micro-sized via architectures for the epoxy matrix should be investigated in regards to minimizing the capacitance loss of multifunctional batteries.

The future aim of this work is to combine the different approaches and fabricate the battery as a continuous part of the composite structure instead of a separate piece, or add on part, to the structure. Results of this research provide designs that could be tailored and fabricated in order to suit specific mechanical and electrochemical requirements in different transportation vessels and mechanical structures. Examples include the wings and fuselage of aircraft, to the exoskeleton of a robotic body, or even the complete interior (composite components) of a highly specialized vehicle. 


\section{References}

[1] J. P. Thomas and M. A. Qidwai, "The Design and Application of Multifunctional Structure-Battery Materials Systems," Journal of the Minerials, Metals and Materials Society, pp. 18-23, 2005.

[2] D. Linden and T. B. Reddy, Handbook Of Batteries: Third Edition, McGraw-Hill, 2001.

[3] M. Yoshio, R. J. Brodd and A. Kozawa, Lithium-Ion Batteries, New York: Springer, 2009.

[4] R. M. Dell, "Batteries fifty years of materials development," Solid State Ionics, pp. 139-158, 2000.

[5] A. Patil, V. Patil, D. W. Shi, J.-W. Choi, D.-S. Paik and S.-J. Yoon, "Issue and challenges facing rechargeable thin film lithium batteries," Materials Research Bulletin, pp. 1913-1942, 2008.

[6] J. Li, C. Daniel and D. Wood, "Materials processing for lithium-ion batteries," Journal of Power Sources, pp. 2452-2460, 2011.

[7] G. Nagasubramanian, "Fabrication and Testing Capabilities for $18650 \mathrm{Li} /(\mathrm{CFx}) \mathrm{n}$ Cells," International Journal of Electrochemical Science, pp. 913-922, 2007.

[8] D. H. Jeon and S. M. Baek, "Thermal modeling of cylindrical lithium ion battery during discharge cycle," Energy Conversion and Management, p. 2973-2981, 2011.

[9] C. A. Vincent, "Lithium batteries: a 50-year perspective, 1959-2009," Solid State Ionics, p. 159-167, 2000.

[10] S. C. Roberts and G. S. Aglietti, "Structural performance of a multifunctional spacecraft structure based on plastic lithium-ion batteries," Acta Astronautica, pp. 424-439, 2010.

[11] P. Liu, E. Sherman and A. Jacobsen, "Design and fabrication of multifunctional structural batteries," Journal of Power Sources, p. 646-650, 2009.

[12] I. H. Oh, S. A. Hong and Y. K. Sun, "Low-temperature preparation of ultrafine LiCoO2 powders by the sol-gel method," Journal of Materials Science, pp. 31773182, 1997. 
[13] S. T. Lee, H. J. Kim, S. D. Choi and S. M. Lee, "The production of LiCoO2 cathode thick films for an all-solid-state microbattery," Journal of Ceramic Processing Research, pp. 106-109, 2007.

[14] X. Qian, X. Cheng, Z. Wang, X. Huang, R. Guo, D. Mao, C. Chang and W. Song, "The preparation of $\mathrm{LiCoO} 2$ nanoplates via a hydrothermal process and the investigation of their electrochemical behavior at high rates," Nanotechnology, pp. 1-7, 2009.

[15] M. S. Park, S. H. Hyun and S. C. Nam, "Characterization of a LiCoO2 thick film by screen-printing for a lithium ion micro-battery," Journal of Power Sources, pp. 14161421, 2006.

[16] Q. Zhang, G. Peng, G. Wang, M. Qu and Z. L. Yu, "Effect of mesoporous carbon containing binary conductive additives in lithium ion batteries on the electrochemical performance of the LiCoO2 composite cathodes," Solid State Ionics, pp. 698-702, 2009.

[17] B. Bitterlich, C. Lutz and A. Roosen, "Rheological characterization of water-based slurries for the tape casting process," Ceramics International, pp. 675-683, 2002.

[18] J. T. Lee, Y. J. Chu, F. M. Wang, C. R. Yang and C. C. Li, "Aqueous processing of lithium-ion battery cathodes using hydrogen peroxide-treated vapor-grown carbon fibers for imporvement of electrochemical properties," Journal of Material Science, pp. 10118-10123, 2007.

[19] C. Iwakura, Y. Fukumoto, H. Inoue, S. Ohashi, S. Kobayashi, H. Tada and M. Abe, "Electrochemical characterization of various metal foils as a current collector of positive electrode for rechargeable lithium batteries," Journal of Power Sources, pp. 301-303, 1997.

[20] K. A. Striebel, A. Sierra, J. Shim, C. -W. Wang and A. M. Sastry, "The effect of compression on natural graphite anode performance and matrix conductivity," Journal of Power Sources, pp. 241-251, 2004.

[21] M. Wang, J. Li, X. He, H. Wu and C. Wan, "The effect of local current density on electrode design for lithium-ion batteries," Journal of Power Sources, pp. 127-133, 2012.

[22] L. Hu, H. Wu, F. L. Mantia, Y. Yang and Y. Cui, "Thin, Flexible Secondary Li-Ion Paper Batteries," American Chemical Society, pp. 5843-5848, 2010. 
[23] G. Liu, H. Zheng and V. S. Battaglia, Fabrication procedure for LiMn2O4/Graphitebased Lithium-ion Rechargeable Pouch Cells, Berkeley, California : BATT Program, Environmental Energy Technologies Division, Lawrence Berkeley National Laboratory, 2007, pp. 1-7.

[24] M. Brain, "http://electronics.howstuffworks.com/everyday-tech/lithium-ionbattery1.htm," 2006. [Online].

[25] M. S. Whittingham, "Lithium Batteries and Cathode Materials," Chemical Reviews, pp. 4271-4301, 2004.

[26] N. Sharma, V. K. Peterson, M. M. Elcombe, M. Avdeev, A. J. Studer, N. Blagojevic, R. Yusoff and N. Kamarulzaman, "Structural changes in a commercial lithium-ion battery during electrochemical cycling: An in situ neutron diffraction study," Journal of Power Sources, pp. 8258-8266, 2010.

[27] M. R. Palacin, "Recent advances in rechargeable battery materials: a chemist's perspective," Chemical Society Reviews, pp. 2565-2575, 2009.

[28] H. Xia, L. Lu, Y. S. Meng and G. Ceder, "Phase Transitions and High-Voltage Electrical Behavior of LiCoO2 Thin Films Gorwn by Pulse Laser Deposition," Journal of The Electrochemical Society, pp. A337-A342, 2007.

[29] C. Peabody and C. B. Arnold, "The role of mechanically induced separator creep in lithium-ion battery capacity fade," Journal of Power Sources, pp. 8147-8153, 2001.

[30] A. Ponrouch and M. R. Palacin, "Optimisation of performance through electrode formulation in conversion materials for lithium ion batteries: $\mathrm{Co} 3 \mathrm{O} 4$ as a case example," Journal of Power Sources, pp. 233-246, 2012.

[31] P. Suresh, A. K. Shukla, S. A. Shivashankar and N. Munichandraiah, "Rechargeable lithium cells with dendrite-free electrodeposited lithium in aluminum as negative electrode," Journal of Power Sources, pp. 166-171, 2004.

[32] N. Balasooriya, P. Touzain and P. Bandaranayake, "Lithium electrochemical intercalation into mechanically and chemically treated Sri Lanka natural graphite," Journal of Physics and Chemistry of Solids, pp. 1213-1217, 2006.

[33] C. Daniel, "Materials and Processing for Lithium-ion Batteries," Journal of Materials, pp. 43-48, 2008.

[34] A. Lewandowski and A. Swiderska-Mocek, "Ionic liquids as electrolytes for Li-ion 
batteries-An overview of electrochemical studies," Journal of Power Sources, pp. 601-609, 2009.

[35] K. Zaghib, X. Song, A. Guerfi, R. Kostecki and K. Kinoshita, "Effect of particle morphology on lithium intercalation rates in natural graphite," Journal of Power Sources, pp. 505-512, 2003.

[36] J. B. Goodenough and Y. Kim, "Challenges for Rechargeable Li Batteries," Chemistry of Materials, pp. 587-603, 2010.

[37] H. Buqa, A. Wursig, D. Goers, L. J. Hardwick, M. Holzapfel, P. Novak, F. Krumeich and M. E. Spahr, "Behaviour of highly crystalline graphites in lithium-ion cells with propylene carbonate containing electrolytes," Journal of Power Sources, pp. 134141, 2005.

[38] C. Wang, A. J. Appleby and F. E. Little, "Irreversiable capacities of graphite anode for lithium ion batteries," Journal of Electroanalytical Chemistry, pp. 9-17, 2002.

[39] A. Ohta, H. Koshina, H. Okuno and H. Murai, "Relationship between carbonaceous materials and electrolyte in secondary lithium-ion batteries," Journal of Power Sources, pp. 6-10, 1995.

[40] W. Bohnstedt, "Aspects of optimizing polyethylene separators," Journal of Power Sources, pp. 234-240, 2001.

[41] S. S. Zhang, "Review on the separators of liquid electrolyte in Li-ion batteries," Journal of Power Sources, pp. 351-364, 2007.

[42] G. Venugopal, J. Moore, J. Howard and S. Pendalwar, "Characterization of microporous separators for lithium-ion batteries," Journal of Power Sources, pp. 3441, 1999.

[43] S. S. Zhang, "The effect of the charing protocol on the cycle life of a Li-ion battery," Journal of Power Sources, pp. 1385-1391, 2006.

[44] H.-H. Lee, Y.-Y. Wang, C.-C. Wan, M.-H. Yang, H.-C. Wu and D.-T. Shieh, "A fast formation process for lithium batteries," Joural of Power Sources, pp. 118-123, 2004.

[45] M. Winter, "The Solid Electrolyte Interphase - The Most Important and the Least Understood Solid Electrolyte in Rechargeable Batteries," Zeitschrift für Physikalische Chemie, pp. 1395-1406, 1009. 
[46] W. Kong, H. Li, X. Huang and L. Chen, "Gas evolution behaviors for several cathode materials in lithium-ion batteries," Journal of Power Sources, pp. 285-291, 2005.

[47] K. H. Lee, E. H. Song, J. Y. Lee, B. H. Jun and H. S. Lim, "Mechanism of gas buildup in a Li-ion cell at elevated temperature," Journal of Power Sources, pp. 201-205, 2004.

[48] P. Novak, J. Ufheil, H. Buqa, F. Krumeich, M. E. Spahr, D. Goers, H. Wilhelm, J. Dentzer, R. Gadiou and C. Vix-Guterl, "The importance of the active surface area of graphite materials in the first lithium intercalation," Journal of Power Sources, pp. 1082-1085, 2007.

[49] H. Buqa, A. Wursig, J. Vetter, M. E. Spahr, F. Krumeich and P. Novak, "SEl film formation on highly crystalline graphitic materials on lithium-ion batteries," Journal of Power Sources, pp. 385-390, 2006.

[50] H. Wang, T. Umeno, K. Mizuma and M. Yoshio, "Highly conductive bridges between graphite spheres to improve the cycle performance of a graphite anode in lithium-ion batteries," Journal of Power Sources, pp. 886-890, 2008.

[51] J. Shim and K. A. Striebel, "Characterization of high-power lithium-ion cells during constant current cycling Part I. Cycle performance and electrochemical diagnostice," Journal of Power Sources, pp. 188-194, 2003.

[52] N. Sharma and V. K. Peterson, "Overcharging a lithium-ion battery: Effect on the LixC6 negative electrode determined by in situ neutron diffraction," Journal of Power Sources, pp. 1-7, 2012.

[53] R. Ruffo, C. Wessells, R. A. Huggins and Y. Cui, "Electrochemical behavior of LiCoO2 as aqueous lithium-ion electrodes," Electrochemistry Communications, pp. 247-249, 2009.

[54] A. J. Smith, J. C. Burns and J. R. Dahn, "A High Precision Study of the Coulombic Efficiency of Li-Ion Batteries," Electrochemical and Solid-State Letters, pp. A177A179, 2010.

[55] E. L. Wong, J. F. Snyder and C. W. Hubbard, "Electrochemical Capacities of Comercially Avaliable Structural Carbon Fibers, Fabrics, and Papers.," U.S Army Research Laboratory, Weapons and Materials Research Directorate, Aberdeen Proving Ground, Maryland, 2008. 
[56] J. F. Snyder, E. L. Wong and C. W. Hubbard, "Evaluation of Commercially Available Carbon Fibers, Fabrics, and Papers for Potential Use in Multifunctional Energy Storage Applications," Journal of The Electrochemical Society, pp. A215A224, 2009.

[57] M. H. Kjell, E. Jacques, D. Zenkert, M. Behm and G. Lindbergh, "PAN-Based Carbon Fiber Negative Electrodes for Structural Lithium-Ion Batteries," Journal of the Electrochemical Society, pp. A1455-A1460, 2011.

[58] Y. J. Kim, H. J. Lee, S. W. Lee, B. W. Cho and C. R. Park, "Effects of sulfuric acid treatment on the microstructure and electrochemical performance of a polyacrylonitrile (PAN)-based carbon anode," Carbon, pp. 163-169, 2005.

[59] J. K. Lee, K. W. An, J. B. Ju, B. W. Cho, W. I. Cho, D. Park and K. S. Yun, "Electrochemical properties of PAN-based carbon fibers as anodes for rechargeable lithium ion batteries," Carbon, pp. 1299-1305, 2001.

[60] R. F. Gibson, "A review of recent research on mechanics of multifunctional composite materials and structures," Composite Structures, pp. 2793-2810, 2010.

[61] H. Chen, X. Qiu, W. Zhu and P. Hagenmuller, "Synthesis and high rate properties of nanoparticled lithium cobalt oxides as the cathode material for lithium-ion battery," Electrochemistry Communications, pp. 488-491, 2002.

[62] A. Lundblad and B. Bergman, "Synthesis of LiCoO2 starting from carbonate precursors II. Influence of calcination conditions and leaching," Solid State Ionics, pp. 183-193, 1997.

[63] J. P. Park, J. Y. Park, C. H. Hwang, M.-h. Chio, J. E. Kim, K. M. Ok and I.-W. Shim, "Synthesis of LiCoO2 Nanoparticles by a Sonochemical Method under the Multibubble Sonoluminescence Conditions," Bulletin of the Korean Chemical Society, pp. 327-330, 2010.

[64] M. Rubin, K. v. Rottkay, S. -J. Wen and O. J. Slacker, "Optical Indicies of Lithiated Electrochromic Oxides," Lawrence Berkeley National Laboratory Publication 39410, pp. 1-6, 1996.

[65] Z. Ma, H. G. Merkus, J. G. d. Smet, C. Heffels and B. Scarlett, "New developments in particle characterization by laser diffraction: size and shape," Powder Technology, pp. 66-78, 2000. 
[66] T. Utsunomiya, O. Hatozaki, N. Yoshimoto, M. Egashira and M. Morita, "Influence of particle size on the self-discharge behavior of graphite electrodes in lithium-ion batteries," Journal of Power Sources, pp. 8675-8682, 2011.

[67] C. Yuqin, L. Hong, W. Lie and L. Tianhong, "Irreversible capacity loss of graphite electrode in lithium-ion batteries," Journal of Power Sources, pp. 187-190, 1997.

[68] Z. Liu, A. Yu and J. Y. Lee, "Modifications of synthetic graphite for secondary lithium-ion battery applications," Journal of Power Sources, pp. 187-191, 1999.

[69] H. Huang, W. Liu, X. Huang, L. Chen, E. M. Kelder and J. Schoonman, "Effect of a rhombohedral phase on lithium intercalation capacity in graphite," Solid State Ionics, pp. 173-178, 1998.

[70] B. Simon, S. Flandrois, K. Guerin, A. Fevrier-Bouvier, I. Teulat and P. Biensan, "On the choice of graphite for lithium ion batteries," Journal of Power Sources, pp. 312316, 1999.

[71] W. Khos, H. J. Santner, F. Hofer, H. Schrottner, J. Doninger, I. Barsukov, H. Buqa, J. Albering, K. C. Moller, J. O. Besenhard and M. Winter, "A study on electrolyte interactions with graphite anodes exhibiting structures with various amounts of rhombohedral phase," Journal of Power Sources, pp. 528-537, 2003.

[72] R. Jenkins and R. L. Snyder, Introduction to X-Ray Powder Diffractometry, John Wiley \& Sons, Inc., 1996.

[73] E. Ligneel, B. Lestriez, A. Hodhomme and D. Guyomard, "Effects of the Solvent Concentration (Solid Loading) on the Processing and Properties of the Composite Electrode," Journal of The Electrochemical Society, pp. A235-A241, 2007.

[74] K.-H. Kim and K.-B. Kim, "Ultrasound assisted synthesis of nano-sized lithium cobalt oxide," Ultrasonics Sonochemistry, pp. 1019-1025, 2008.

[75] V. Palomares, A. Goni, I. G. d. Muro, I. d. Meatza, M. Bengoecha, I. Cantero and T. Rojo, "Conductive additive content balance in Li-ion battery cathodes: Commercial carbon black vs. in situ carbon from LiFePO4/c composites," Journal of Power Sources, pp. 7661-7668, 2010.

[76] D. Hotza and P. Greil, "Review: aqueous tape casting of ceramic powders," Materials Science and Engineering, pp. 206-217, 1995.

[77] J.-H. Lee, S.-B. Wee, M.-S. Kwon, H.-H. Kim, J.-M. Choi, M. S. Song, H. B. Park, H. 
Kim and U. Paik, "Strategic dispersion of carbon black and its applications to inkjet-printed lithium cobalt oxide electrodes for lithium ion batteries," Journal of Power Sources, pp. 6499-6455, 2011.

[78] R. Janot and D. Guerard, "Ball-milling in liquid media Applications to the preparation of anodic materials for lithium-ion batteries," Progress in Materials Science, pp. 1-92, 2005.

[79] G. Park, N. Gunawardhana, H. Nakamura, Y.-S. Lee and M. Yashio, "The study of electrochemical properties and lithium deposition of graphite at low temperature," Journal of Power Sources, pp. 293-299, 2012.

[80] A. Bismark, C. Wuertz and J. Springer, "Basic surface oxides on carbon fibers," Carbon, pp. 1019-1027, 1999.

[81] T. Ijima, K. Suzuki and Y. Matsuda, "Electrodic characteristics of various carbon materials for lithium rechargeable batteries," Synthetic Metals, pp. 9-20, 1995.

[82] M. S. Rahaman, A. F. Ismail and A. Mustafa, "A review of heat treatment on polyacrylonitrile fiber," Polymer Degradation and Stability, pp. 1421-1432, 2007.

[83] Z. Dai, F. Shi, B. Zhang, M. Li and Z. Zhang, "Effect of sizing on carbon fiber surface properties and fibers/epoxy interfacial adhesion," Applied Surfacce Science, pp. 6980-6985, 2011.

[84] K. Naito, Y. Tanaka, J.-M. Yang and Y. Kagawa, "Tensile properties of ultrahigh strength PAN-based, ultrahigh modulus pith-based and high ductility pitch-based carbon fibers," Carbon, pp. 189-195, 2008.

[85] X. Qin, Y. Lu, H. Xiao, Y. Wen and T. Yu, "A comparison of the effect of graphitization on microstructures and properties of polyacrylonitrile and mesophase pitch-based carbon fibers," Carbon, pp. 4459-4469, 2012.

[86] B. Lindsay, M.-L. Abel and J. F. Watts, "A study of electrochemically treated PAN based carbon fibres by IGC and XPS," Carbon, pp. 2433-2444, 2007.

[87] W. H. Lee, J. G. Lee and P. J. Reucroft, "XPS study of carbon ${ }^{\circledR} b e r$ surfaces treated by thermal oxidation in a gas mixture of O2/(O2+N2)," Applied Surface Science, pp. 136-142, 2001.

[88] E. Pamula and P. G. Rouxhet, "Bulk and surface chemical functionalities of type III PAN-based carbon fibers," Carbon, pp. 1905-1915, 2003. 
[89] Y. V. Basova, H. Hatori, Y. Yamada and K. Miyashita, "Effect of oxidation-reduction surface treatment on the electrochemical behavior of PAN-based carbon fibers," Electrochemistry Communications, pp. 540-544, 1999.

[90] W. Shen, Z. Li and Y. Liu, "Surface Chemical Functional Groups Modification of Porous Carbon," Recent Patents on Chemical Engineering, pp. 27-40, 2008.

[91] S. Wang, Z.-H. Chen, W.-J. Ma and Q.-S. Ma, "Influence of heat treatment on physical-chemical properties of PAN-based carbon fiber," Ceramics International, pp. 291-295, 2006.

[92] P. Xiao, Z. Q. Deng, A. Manthiram and G. Henkelman, "Calculations of Oxygen Stability in Lithium-Rich Layered Cathodes," Journal of Physical Chemistry, p. 2012, 23201-23204.

[93] F. C. Campbell, Structural Composite Materials, United States: ASM International, 2010.

[94] West System, Vacuum Bagging Techniques 002-150, Bay City, MI: Gougeon Brothers Inc., 2010.

[95] E. Sahraei, R. Hill and T. Wierzbicki, "Calibration and finite element simulation of pouch lithium-ion batteries for mechanical integrity," Journal of Power Sources, pp. 307-321, 2012.

[96] L. Greve and C. Fehrenbach, "Mechanical testing and macro-mechanical finite element simulation of the deformation, fracture, and short circuit of cylindrical Lithium ion battery cells," Journal of Power Sources, pp. 377-385, 2012.

[97] J. Lamb and C. J. Orendorff, "Evaluation of mechanical abuse techniques in lithium ion batteries," Journal of Powder Sources, pp. 189-196, 2014.

[98] Z. K. Award, T. Aravinthan and A. Manalo, "Geometry effect on the behaviour of single and glue-laminated glass fibre reinforced polymer composite sandwich beams loaded in four-point bending," Materials and Design, pp. 93-103, 2012.

[99] A. C. Manalo, T. Aravinthan, W. Karunasena and M. M. Islam, "Flexural behaviour of structural fibre composite sandwich beams in flatwise and edgewise positions," Composite Structures, pp. 984-995, 2010. 


\section{Appendix A}

Split cell initial carbon fiber anode and fiberglass separator performance data.
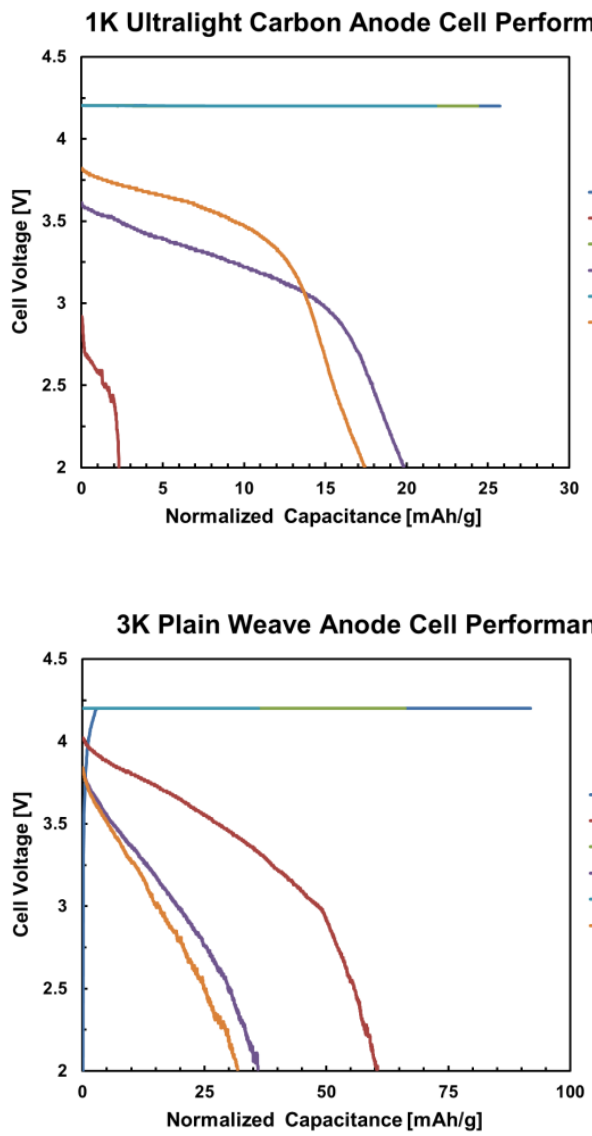
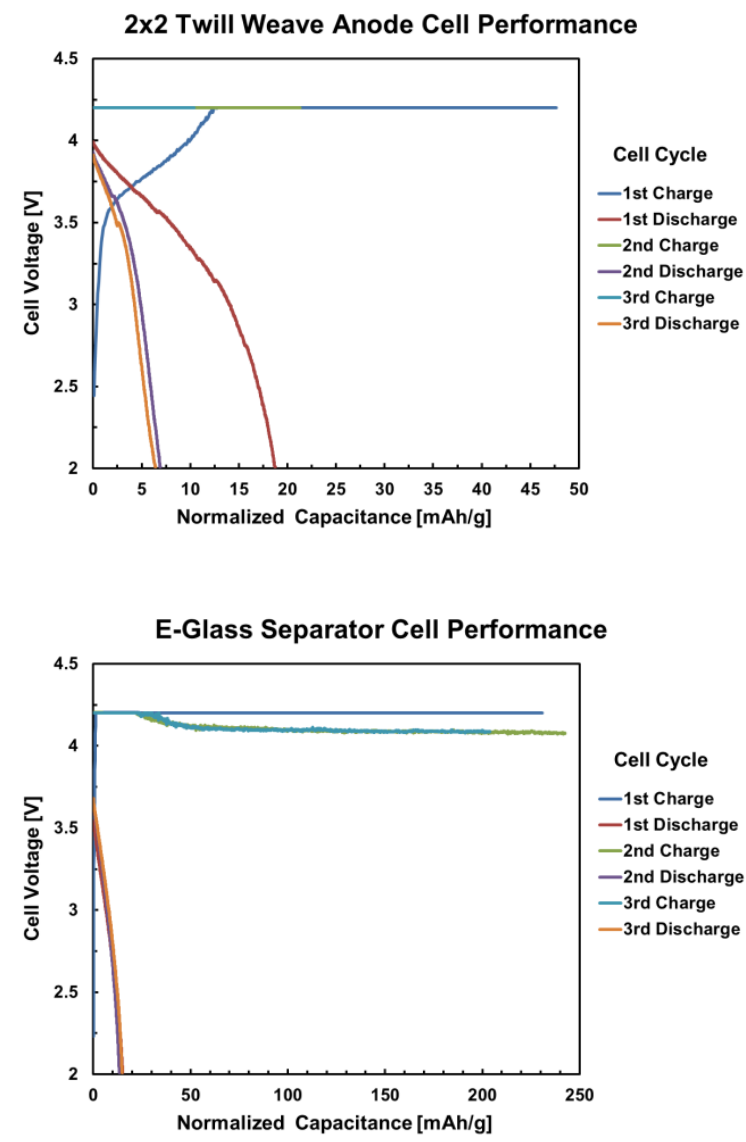